\title{
Models for spatial organization of microtubules and cell polarization
}




\section{Thesis committee}

\section{Promotor}

Prof. Dr Bela M. Mulder

Professor of Theoretical Cell Physics

Wageningen University \& Research

Group leader Theory of Biomolecular Matter

Institute AMOLF, Amsterdam

\section{Other members}

Prof. Dr Frans Leermakers, Wageningen University \& Research Dr Eva Deinum, Wageningen University \& Research Prof. Dr Pieter Rein ten Wolde, VU University, Amsterdam Prof. Dr René van Roij, Utrecht University 


\title{
Models for spatial organization of microtubules and cell polarization
}

\author{
Panagiotis Foteinopoulos
}

\section{Thesis}

submitted in fulfilment of the requirements for the degree of doctor

at Wageningen University

by the authority of the Rector Magnificus

Prof. Dr A.P.J. Mol,

in the presence of the

Thesis Committee appointed by the Academic Board

to be defended in public

on Thursday 12 September 2019

at 11 a.m. in the Aula. 
Panagiotis Foteinopoulos

Models for spatial organization of microtubules and cell polarization 210 pages.

PhD thesis, Wageningen University, Wageningen, The Netherlands (2019)

With references, with summaries in Dutch and English

ISBN: 978-94-6395-004-6

DOI: https://doi.org/10.18174/478734 
To my wife 



\section{Contents}

$\mathbf{1}$ Introduction $\mathbf{5}$

1.1 Biological context . . . . . . . . . . . . . . . . 6

1.1.1 Understanding the living cell . . . . . . . . . . . 6

1.1.2 Microtubule-based cellular modules. . . . . . . . . . 6

1.1.3 The microtubule cytoskeleton . . . . . . . . . . 7

1.2 Our focus: how cell shape controls microtubule organization 11

1.3 Modelling microtubules $\ldots \ldots \ldots \ldots$

1.3 .1 Analytical approach . . . . . . . . . . . . . . . . . . 13

1.3 .2 Stochastic simulations . . . . . . . . . . . . 15

1.4 Outline . . . . . . . . . . . . . . . . 16

\begin{tabular}{lll}
\hline I Branched Nucleation & 19
\end{tabular}

$\begin{array}{|ll|}2 & \text { Anisotropic microtubule-bound nucleations }\end{array}$

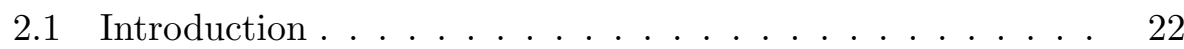

2.2 Theoretical framework . . . . . . . . . . . . . . . . . . . . 24

2.2 .1 Basic theory . . . . . . . . . . . . . . . . 24

2.2 .2 Binding equilibrium of the nucleation complexes . . 28

$2.2 .3 \quad$ Anisotropic distribution of nucleation angles. . . . . 29

2.2 .4 Dimensional analysis . . . . . . . . . . . . . 31

2.3 Results . . . . . . . . . . . . . . . . . . . . . . . . . . . 33

$2.3 .1 \quad$ Bifurcation analysis $\ldots \ldots \ldots \ldots . \ldots . \ldots 33$

2.3 .2 Numerical solutions . . . . . . . . . . . . . . . 36

2.3 .3 Comparison to simulations . . . . . . . . . . . . . . . . . . . . . . . . . .

2.4 Conclusion $\ldots \ldots \ldots \ldots \ldots$ 


\section{Microtubule-based phenomena induced by shape}

$\begin{array}{|ll|}3 & \text { Cell Shape }\end{array}$

$3.1 \quad$ Introduction . . . . . . . . . . . . . . . . . . . 46

$3.2 \quad$ Model . . . . . . . . . . . . . . . . . . . . . . . . . . 47

3.3 Results . . . . . . . . . . . . . . . . . . . . . . . . . . . 54

3.3.1 General ellipsoidal cell with a central centrosome . . 54

$3.3 .2 \quad$ Axially symmetric cell with a central centrosome . . 56

3.3 .3 Non-central nucleation . . . . . . . . . . . . . 63

3.4 Conclusion $\ldots \ldots \ldots \ldots$

\begin{tabular}{|lll}
4 & A microtubule boundary-sliding model & 69
\end{tabular}

4.1 Introduction . . . . . . . . . . . . . . . . 70

4.2 The model . . . . . . . . . . . . . . . . . . . . . . . . . . . 71

$4.2 .1 \quad$ Force-production model $\ldots \ldots \ldots \ldots$. . . . . . . . 71

$4.2 .2 \quad$ Evolution equations of sliding microtubules . . . . . 74

4.3 Analytical results . . . . . . . . . . . . . . . . . . . . 77

4.4 Simulations . . . . . . . . . . . . . . . . . . . . . . . . . 79

4.5 Conclusion $\ldots \ldots \ldots \ldots$

4.6 Appendix $\ldots \ldots \ldots \ldots \ldots \ldots$

4.6 .1 Expansions for the evolution equations. . . . . . . 85

4.6 .2 Perturbation analysis $\ldots \ldots \ldots \ldots$. . . . . . 86

\begin{tabular}{|lll}
5 & Mitotic spindle positioning in cells & 91
\end{tabular}

5.1 Introduction . . . . . . . . . . . . . . . . . . 92

5.2 The model . . . . . . . . . . . . . . . . . . . . . . . . 92

$5.2 .1 \quad$ Structure of the spindle complex $\ldots \ldots \ldots$. . . . 92

5.2 .2 Equations of motion $\ldots \ldots \ldots \ldots . \ldots 94$

5.3 Simulations . . . . . . . . . . . . . . . . . 95

5.3 .1 Simulation model . . . . . . . . . . . . . . . . 95

5.3 .2 Simulation geometries . . . . . . . . . . . . . 97

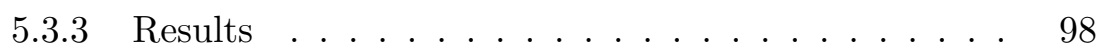

5.4 Conclusion $\ldots \ldots \ldots \ldots \ldots$

5.5 Appendix: Relations between the drag coefficients . . . . 106 
\begin{tabular}{lll}
\hline III Cell polarity & 109
\end{tabular}

6 Spherical cell polarity: a minimal model 111

6.1 Introduction . . . . . . . . . . . . . . . . . . . . . . 112

6.2 The Model . . . . . . . . . . . . . . . . . . . . . . . 113

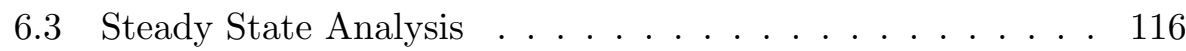

6.4 Bifurcation Analysis Results . . . . . . . . . . . . . . . 120

6.5 Simulation Method . . . . . . . . . . . . . . . . . . . . 123

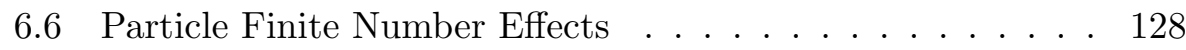

6.7 Effect of a Finite Tubulin Pool $\ldots \ldots \ldots$

$6.7 .1 \quad$ Dynamical equations . . . . . . . . . . . . . . . . . . 130

6.7 .2 Steady state solution . . . . . . . . . . . . . . . . . . . 132

6.8 Conclusion $\ldots \ldots \ldots \ldots \ldots \ldots \ldots \ldots \ldots$

6.9 Appendix . . . . . . . . . . . . . . . . . . . 135

$6.9 .1 \quad$ Properties of the isotropic solution . . . . . . . . 135

6.9 .2 Requirements on the dose-response function . . . . . 136

6.9 .3 Proof of the Finite Tubulin Pool Inequalities . . . . 136

$\begin{array}{lll}7 & \text { Polarization in non-spherical cells } & 139\end{array}$

7.1 Introduction . . . . . . . . . . . . . . . . . . 140

7.2 Simulation details $\ldots \ldots \ldots \ldots$

7.2 .1 Implementing and validating diffusion . . . . . . . . 141

$\begin{array}{lll}7.2 .2 & \text { Force production in presence of polarization factors. } & 146\end{array}$

$7.2 .3 \quad$ Order parameters . . . . . . . . . . . . . . . . . . . 148

7.3 Results . . . . . . . . . . . . . . . . . . . . . . . . . 149

$7.3 .1 \quad$ Polarization in the absence of sliding . . . . . . . . . 149

7.3 .2 Polarization in the presence of sliding . . . . . . . . 155

7.3.3 Analytical model of perfect bipolar biasing . . . . . 161

7.4 Conclusion . . . . . . . . . . . . . . . . . 167

\begin{tabular}{lll}
\hline IV General discussion & 169
\end{tabular}

8 Conclusion and Outlook 171

8.1 Branched versus background nucleation . . . . . . . . . . 172

8.2 Microtubule organization induced by shape . . . . . . . . . 172

$8.2 .1 \quad$ Stalling at the cell boundary . . . . . . . . . . . . 172

$8.2 .2 \quad$ Sliding on the cell boundary . . . . . . . . . . 174 
Contents

8.2.3 Positioning the mitotic spindle . . . . . . . . . . . . 175

8.3 Microtubule-based cell polarity . . . . . . . . . . . . . . 176

8.3 .1 Spherical cell . . . . . . . . . . . . . . . . . . . 176

$8.3 .2 \quad$ Non-spherical cell. . . . . . . . . . . . . . . . . 178

\begin{tabular}{ll}
\hline Summary & 191
\end{tabular}

$\begin{array}{ll}\text { Samenvatting } & 195\end{array}$

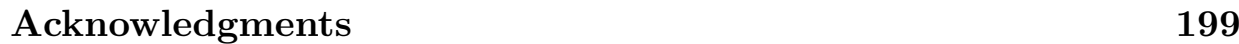

\begin{tabular}{ll}
\hline List of publications & 201
\end{tabular}

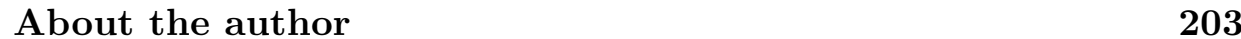




\section{1 \\ Introduction}

In this chapter we introduce microtubules and present their properties, as well as their functions in the cell. We address questions that concern a wide range of microtubule-based phenomena from their spatial organization up to the establishment of cell polarity. We make a short reference to the models to be developed in order to give answers to the questions addressed. Finally, we give an outline of the content of each chapter as well as the analytical and simulation techniques that will be applied. 


\subsection{Biological context}

\subsubsection{Understanding the living cell}

Cells are highly complex systems with many processes occurring simultaneously, which makes their study as whole units highly challenging. Nevertheless, many labs worldwide are currently attempting various forms of whole-cell modelling. The initiatives in this direction typically include the development of electronic platforms, which incorporate information concerning the metabolic pathways, as well as gene regulation and expression [1]. In this way, many biochemical reaction networks have been implemented.

However, these whole-cell modelling approaches typically do not consider the spatial dynamics of cellular structures, although these may may become part of it in the future [2]. So, basic physical and biochemical effects which are spatially dependent, such as localization, spatially extended structuring and even motion of cellular components, are not analyzed in these approaches.

It is for these types of questions that the so-called modular approach to cell biology has proved highly effective. This approach starts from identifying functional modules [3]: a finite set of intracellular parts that together, and mainly in isolation of other components, perform a distinct biological function. The study of such modular functions is based on three complementary approaches: (i) The development of better methods for monitoring dynamic processes in cells, (ii) the synthetic biology approach of reconstituting modular parts or building new ones in a controlled in vitro setting and (iii) the establishment of modelling frameworks that to complement the experiments opening the road to full mechanistic understanding.

In the current thesis we will adopt the latter approach, and explore a number of cellular modules, with the common thread that they are all based on the dynamical behaviour of microtubules.

\subsubsection{Microtubule-based cellular modules}

The main actors in the present study are microtubules, their regulatory environment, and their individual and collective behaviour. Microtubules are the one of the three kinds of filamentous protein aggregates that constitute the cellular structure called the cytoskeleton, the other two being actin and 
the intermediate filaments 4 .

The cytoskeleton plays many important roles in the cell. As its name already suggests, one of these is to control the overall cell shape [5]. Another major task fulfilled by the cytoskeleton is the intracellular transport of vesicles to the cell membrane, where the latter may be incorporated through a process called exocytosis [6]. The transport is achieved by motor proteins that bind on one of their ends to vesicles and with the other end "walk" on the cytoskeletal filaments towards the cell boundary. The resulting asymmetric spatial organization of vesicles and, generally, cellular components at the cell boundary is known as cell polarization. This polarization is essential to many basic processes of the cell, like cell division, cell differentiation, cell migration, cell-cell signaling and morphogenesis.

Here, we apply the modular approach and study four cytoskeletal modules all based on microtubule. These are the plant cortical microtubule array, the organization of the interphase radial microtubule array in animal cells, the localization dynamics of the fully formed mitotic spindle, and microtubule-based cell polarization mechanisms.

\subsubsection{The microtubule cytoskeleton}

\subsubsection{Structure and dynamics of microtubules}

Microtubules are the stiffest polymers of the cytoskeleton, with a persistence length of $\approx 1 \mathrm{~mm}[7$, implying that unless actively deformed they are effectively rigid on the typical size scale of cells. At the same time they can reach lengths in the order of $\sim 10 \mu m$, allowing them to be sensitive to the geometry of the cell. The structural units of microtubules are heterodimers of $\alpha$ and $\beta$ tubulin. The selective $\alpha$-to- $\beta$ longitudinal end-to-end binding of these dimers leads to straight protofilaments, thirteen of which are positioned side by side to form a hollow cylinder with an outer diameter of $25 \mathrm{~nm}$. At one end (denoted by -) of a protofilament the $\alpha$ subunits of the dimers are exposed, while at the other end (denoted by + ) it is the $\beta$ subunits that are exposed. Due to this fact, a microtubule has an intrinsic structural polarity, which is basic for its biological function. Specifically, among the many microtubule associated proteins (MAPs) 8] there are motor proteins, like kinesins and dyneins, which can walk on microtubules exclusively in one direction taking advantage of this structural polarity. This enables directionally selective vesicle transport to the cell membrane 
by these motor proteins, opening the way for the cell polarization.

A key property of microtubules is their highly dynamic character. They can switch stochastically from a state of growth to that of shrinkage - an event called a catastrophe — and the other way round - in an event called a rescue - . This intrinsic property of microtubules is known as dynamic instability 9. The growth of microtubules depends on the availability of GTP-bound tubulin. Indeed, only GTP-bound tubulin dimers can drive polymerisation through attachment at the microtubule +-end [10] and the presence of a "cap" of GTP-tubulin at this end is necessary for growth. Once incorporated into the microtubule lattice the tubulin-bound GTP can be hydrolyzed to GDP. It is thought that a catastrophe occurs when through a statistical fluctuation the microtubule cap is fully hydrolyzed 11. At present our understanding of the mechanism underlying the rescue event is almost non-existent. The continuous turn-over of GTP which drives this coupled growth-shrinkage process indicates that microtubule dynamics is a far out-of-equilibrium process. The fact that microtubules can shrink to zero length following a catastrophe, implies that maintaining a stable population of microtubules also requires their (re)nucleation as an essential process.

\subsubsection{Cellular organization and function of microtubules}

The first microtubule-based cellular module that we describe is the cortical microtubule array in growing interphase plant cells. While in animal cells microtubules are typically nucleated from a single microtubule organizing center (MTOC) [12], such a structure is absent in plant cells [13]. The resultant type of microtubule organisation is therefore referred to as noncentrosomal. The plant cortical microtubules are nucleated from nucleation complexes that are mainly attached to pre-existing microtubules [14, 15. Both new and parent microtubules are all attached to the cell membrane with linking proteins [16] and together form the cortical array (see figure 1.1. The attachment to the membrane creates an effectively $2 \mathrm{D}$ environment for the interactions between cortical microtubules. These interactions have been observed in the form of frequent collisions of the tips of growing microtubules with other microtubules [17]. These collisions can collectively lead to spontaneous alignment of the microtubule population, as has been shown e.g. by computer simulations [18. The correct orientation through mutual alignment of the plant cortical array is essential for proper plant 
morphogenesis as it regulates the oriented deposition of cellulose in the plant cell wall [19, which in turn determines the direction of cell growth.

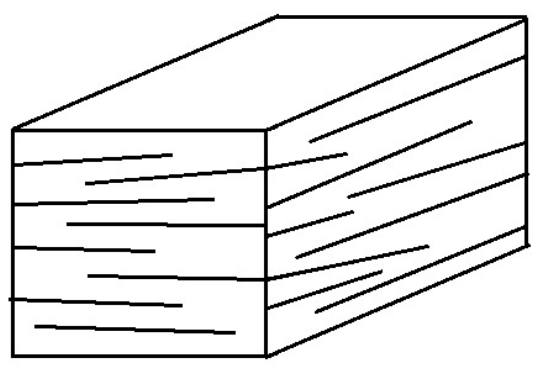

Figure 1.1: Schematic of the plant cortical array. We can see the alignment of the cortical microtubules on the facets of the plasma membrane under the cell wall.

The second module that we'll be studying is the interphase radial array in animal cells. This is an array of microtubules that are nucleated by a single centrosome and extend radially towards the cell boundary. The microtubule radial array supports the transport of vesicles to the plasma membrane with the help of motor proteins that move along the microtubules of its network.

The third cellular module that we'll examine is the localization dynamics of the mitotic spindle, which plays a crucial role in cell division. The interaction of microtubules with the cell boundary generates forces which lead to the positioning of the mitotic spindle in a way that down-stream enables the correct spatial segregation of the daughter chromosomes. Within the mitotic spindle, microtubules fulfill three different main functions. The kinetochore microtubules connect the spindle poles with the chromosomes, the interpolar microtubules connect the two poles and support the kinetochore microtubules and, finally, the astral microtubules connect the spindle poles with the plasma membrane. 


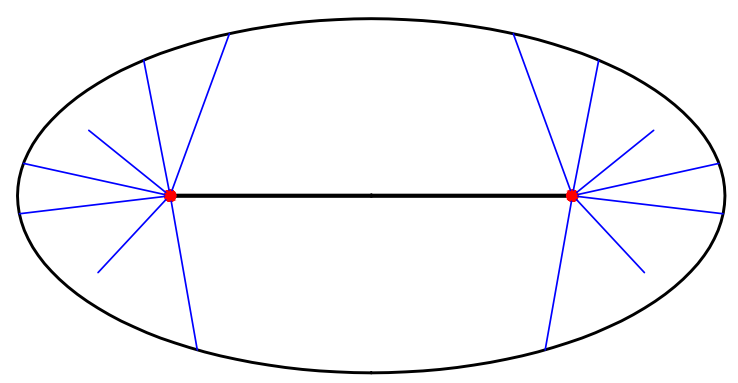

Figure 1.2: Schematic of the mitotic spindle. We can see the two centrosomes (red circles) and the radial array of astral microtubules (blue line segments) nucleated by them during cell division. Astral microtubules stabilize the spindle by interacting with the cell boundary (black elliptical border). The black line segment stands for the kinetochore and interpolar microtubules.

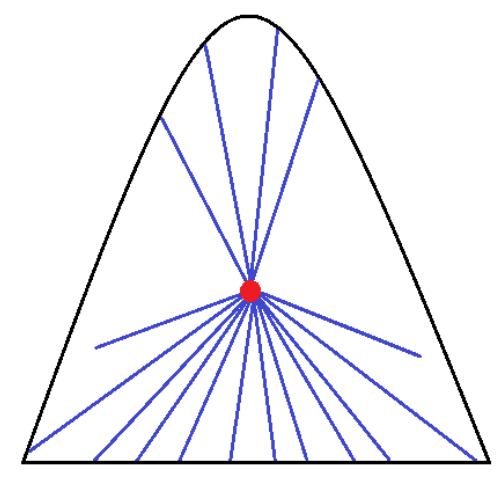

Figure 1.3: Schematic of a polarized fibroblast. Most of the microtubules (blue line segments) that are nucleated from the centrosome (red circle) bind to the flat cell edge, which supports the directed migration of the fibroblast.

The fourth module that we'll investigate are microtubule-based cell polarization mechanisms. The spatial organization of centrosomal micro- 
tubules as determined by their intrinsic dynamics in concert with the cell shape, is a first step for the establishment of polarity in animal cells (see figure 1.3). The ability of microtubules to bind and selectively transport proteins to the plasma membrane is the property that opens a route to cell polarization. As already mentioned, the asymmetric distribution of intracellular components is of key importance to many developmental processes in biology.

\subsection{Our focus: how cell shape controls micro- tubule organization}

In the present thesis we develop a series simple minimalistic models for the four microtubule-based cellular modules described above. A red thread running through all these models is the question of how the finite size and shape of cells influences the spatial organization of microtubules.

We start with the plant cortical microtubule array. In this case the role of cell shape is still implicit, as it only serves to select the global orientation of the aligned microtubules [20]. Instead, we focus on the influence of the unique directionality of microtubule-bound microtubule nucleation that occur. Simulations have shown that these can strongly impact the degree of ordering of the array [21], but a proper theoretical basis was lacking. We focus on the competition between the anisotropic microtubule-bound nucleation and the background isotropic nucleation in the bulk, which we hypothesize to be strong homeostatic mechanism for robust microtubule alignment.

On the other hand, in animal cells the spatial organization of microtubules originates from the centrosome. While microtubules interact with other intracellular components such as MAPS, organelles, and, during mitosis, with chromosomes, the strongest geometrical constraint on their behaviour is likely due to interactions with the cell boundary. This raises the simple, but hitherto less explored, question of how cell shape by itself influences the spatial distribution of microtubules. Under certain conditions [22], microtubules hitting the cell boundary can stop growing and remain stalled there for a variable duration. A key question in this case is how can we predict the length distribution of microtubules as a function of the orientation with respect to the axes of the cell. However, if microtubules continue to grow after hitting the cell boundary, pushing forces are gener- 
ated that feed back on the microtubules. Therefore, when the boundary is not spherical, the microtubules can slide slide along the cell surface surface. The pushing forces have a dynamic nature as they are explicitly related to the growth speed and the catastrophe rate of microtubules at the boundary. A key question at this level is how can we build a model that predicts the motion of the sliding microtubule ends along the cell boundary by properly incorporating the dynamic nature of the pushing forces.

It is known that astral microtubules are nucleated from the two poles of the mitotic spindle and, subsequently, interact with the plasma membrane leading to the positioning of the spindle. A few models for the spindle positioning have been presented to date [22, 23, 24, 25], but so far the dynamic nature of the pushing forces has not been taken into consideration. We aim to formulate a model that predicts the spindle motion and positioning by using a self-consistent dynamic force production mechanism taking place at the cell boundary. Earlier work on positioning has pointed to an important role in positioning for pulling forces generated on the tips of microtubules [26. 23], so these will be included as well. The questions we address are how the model parameters contribute to the robustness ans selectively of the positioning of the spindle within the cell volume.

In setting up models for the fourth module we consider, we choose to adopt a synthetic biology approach. Instead, of looking at existing polarization mechanisms in vivo, we are interested in building a model that can provide a route towards the creation of cell polarity in a minimal cell-like environment. Currently, there is a world-wide drive towards building such synthetic cells. Of special interest to us the so-called bottom-up approach [27, in which in an opportunistic fashion different cellular modules, based on or inspired by existing ones from different organisms, are combined to form a functional whole. In that context it is interesting to ask how a polarization mechanism can be built using the minimal number of available components. From a theoretical point of view it is then important to provide proof of principle for the most difficult case, that of a perfectly spherical cell. However, generically cells are not spherical, and therefore we must also address how cell geometry modulates our candidate polarization mechanism. 


\subsection{Modelling microtubules}

Since we are interested in modelling biological phenomena that place on a time scale of minutes to hours in cells that have a typical size of a few to tens of $\mu \mathrm{m}$ 's, we use a mesoscopic model in order to represent microtubule molecules [28]. We therefore do not regard the internal atomic structure of a microtubule or any other processes at the atomic scale, like the binding of individual nm-sized tubulin dimers to the microtubule end, with which sub-millisecond timescales are associated [29]. Rather we treat the macromolecule as a whole as a single object, described by small set of relevant observables. The choice of mesoscopic representation enables the treatment of the microtubule dynamics using both semi-deterministic analytical models and particle-based stochastic simulations described below.

\subsubsection{Analytical approach}

Our analytical modelling is based on the original dynamic instability model introduced by Dogterom and Leibler [9]. According to this model, every microtubule is a rigid rod with a 'plus' end that is either growing with a speed $v_{+}$or is shrinking with a speed $v_{-}$. The 'plus' end can also switch stochastically from a growing to a shrinking state (a catastrophe) with rate $r_{+}$, or from a shrinking to a growing state (a rescue) with rate $r_{-}$. A nucleation process must also be included. Here we present a variant of the original model with a fixed number overall number of microtubules, each of which is associated with a nucleation complex. We call a not yet nucleated microtubule dormant. It is activated from this state with a constant rate $r_{n}$. When a microtubule shrinks to zero length, it reenters the dormant state. The observables in this model are the densities $m^{+}(l, t)$ and $m^{-}(l, t)$ representing the average number of growing and shrinking microtubules of length $l$ at time $t$ respectively and $m_{0}(t)$ the number of dormant microtubules. These quantities satisfy the following set of dynamical equations, which in essence describe the conservation of total tubulin "mass"

$$
\begin{aligned}
\partial_{t} m^{+}(l, t) & =-v_{+} \partial_{l} m^{+}(l, t)+r_{-} m^{-}(l, t)-r_{+} m^{+}(l, t) \\
\partial_{t} m^{-}(l, t) & =v_{-} \partial_{l} m^{-}(l, t)-r_{-} m^{-}(l, t)+r_{+} m^{+}(l, t) \\
\partial_{t} m_{0}(t) & =-r_{n} m_{0}(t)+v_{-} m^{-}(l=0, t)
\end{aligned}
$$


where $\partial_{x}$ denotes a partial derivative with respect to the variable $x$. In the steady state Eqs.11.1, 1.2, 1.3 reduce to

$$
\begin{aligned}
v_{+} \partial_{l} m^{+}(l) & =r_{-} m^{-}(l)-r_{+} m^{+}(l) \\
-v_{-} \partial_{l} m^{-}(l) & =-r_{-} m^{-}(l)+r_{+} m^{+}(l) \\
v_{+} m^{+}(l=0) & =r_{n} m_{0}
\end{aligned}
$$

Adding Eqs. (1.4) and (1.5) gives

$$
\partial_{l}\left\{v_{+} m^{+}(l)-v_{-} m^{-}(l)\right\}=0
$$

The physically acceptable solutions should be bounded as the microtubule length goes to infinity, which dictates the integration constant to vanish. This yields the balance equation

$$
v_{+} m^{+}(l)=v_{-} m_{i}^{-}(l)
$$

Then, we can eliminate $m^{-}(l)$ in Eq. 1.4$)$ and solve this equation using Eq. 1.6

$$
m^{+}(l)=\frac{r_{n}}{v_{+}} m_{0} e^{-l / \bar{l}}
$$

where

$$
\bar{l}=\left(\frac{r_{+}}{v_{+}}-\frac{r_{-}}{v_{-}}\right)^{-1}>0
$$

is the mean length of microtubules. This quantity has a finite positive value in the bounded-growth regime, which requires the inequality $r_{+} v_{-}-r_{-} v_{+}>$ 0 to hold. [9]. Throughout this is the regime that we work in, which is justified as the cellular environment has a finite volume and, therefore, contains a finite amount of tubulin. Even if the intrinsic dynamical parameters put the microtubules in the unbounded growth regime, the growth speed will eventually be decreased by a shortage of free tubulin available for polymerization. Hence, in steady state the microtubules will effectively always be in the bounded growth regime. A final characteristic parameter of the microtubule dynamics is the mean lifetime [30]

$$
\bar{t}=\bar{l}\left(\frac{1}{v_{+}}+\frac{1}{v_{-}}\right)
$$


which is the expected lifetime of a newly nucleated microtubule.

In the rest of the thesis the model described here will be extended with the necessary ingredients to describe the specific phenomena we are interested in.

\subsubsection{Stochastic simulations}

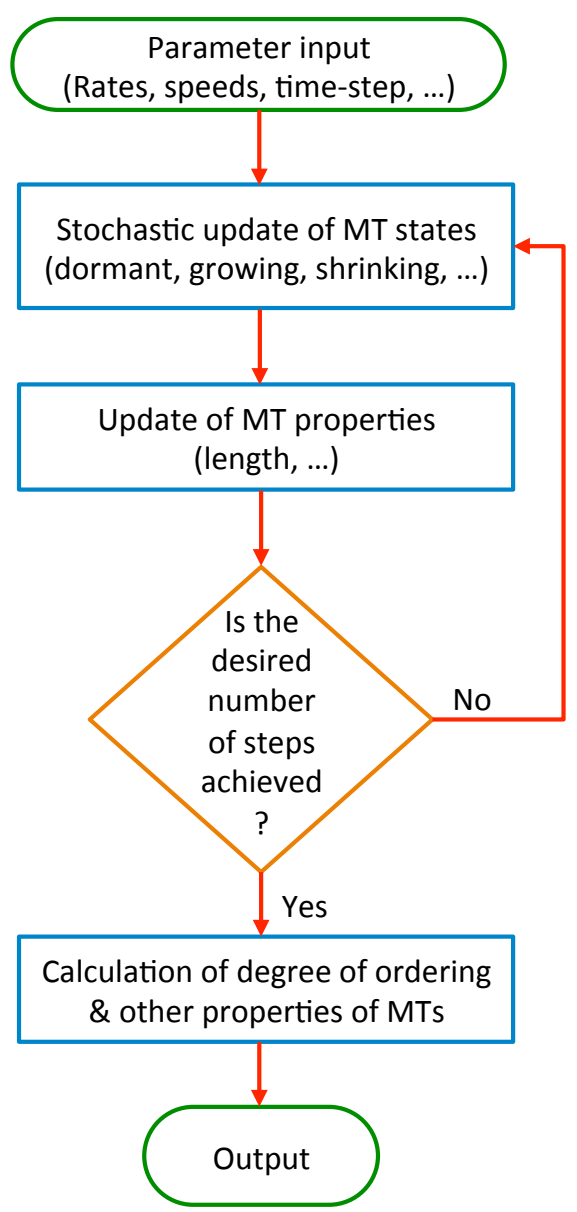

Figure 1.4: Flowchart of the basic stochastic simulation algorithm applied in the present thesis (MT implies microtubule). 
The use of a mesoscopic model for representing microtubules makes possible the application of particle-based stochastic simulations either for verifying our analytical results or analyzing microtubule-based models that are complex to investigate analytically. In our simulations each individual microtubule is described as being in specific dynamical state (in the simplest model GROWING, SHRINKING and DORMANT) and having certain properties (in the simplest case just a length $l$ ). The microtubules evolve in time by taking small discrete time steps $\Delta t$. This time step needs to be taken small enough such that the probability of a state-changing event (in the simple model a catastrophe, a rescue, or a nucleation event) which is given by the product rate $\times$ time step, is so small (typically less the $10^{-2}$ ) that the probability of two events happening in the time step is negligible. In each cycle of the simulation each microtubule is considered in turn. Depending on its state a random number is drawn and compared to the probability of the relevant state-changing event. If it the event is accepted, the state changes. Otherwise it continues in its present state, and its properties, e.g. a change of length due to growth or shrinkage, are updated. After a desired number of time-steps is executed, relevant statistical quantities, such as steady-state length distributions, are calculated and stored. A flowchart of the simulation algorithm is shown in Figure 1.4 .

\subsection{Outline}

The first part (chapter 2) of this thesis is about the role of the branched nucleation of microtubules in ordering in the plant cortical array. Using an analytical approach in the context of a mean-field theory, we reveal a single parameter that determines the relative influence of the microtubule-bound nucleation to the background nucleation. Applying bifurcation analysis, we find the main determinant parameter that leads the system to order. We confirm the robustness of our mean-field theory by comparing with particle-based simulations.

The second part of the thesis is about the spatial organization of microtubules that is induced by the cell shape. In chapter 3 we develop analytical relations predicting the distribution of centrosomal microtubules inside a $3 \mathrm{D}$ confinement and we validate them by $2 \mathrm{D}$ simulations. The distribution of non-centrosomal microtubules inside an elliptical boundary is also calculated, by numerical analysis. 
In chapter 4 we develop a boundary-sliding model that predicts the distribution of centrosomal microtubules, which slide on a non-spherical cell boundary. The force-production mechanism that is used generates pushing forces of dynamic nature by following the Hooke's law. We solve the complex equations of the model by considering a small perturbation to the sphere and we prove the existence of a preferential ordering direction of microtubules stalled to the boundary. We verify the establishment of this ordering by applying 2D stochastic simulations.

In chapter 5 we formulate a model that predicts the positioning of the mitotic spindle in cell. We apply the force-production mechanism that we referred previously, leaving apart the sliding effect, but adding the pulling forces acting by dynein motors at the boundary. By performing computer simulations to an elliptical cell, we reveal the central role that pulling forces play in the spindle stabilization. Furthermore, for the cell shape of a halfellipse, we show how the axes ratio determine the transition between two orientation states of the spindle.

The third part of this thesis is about a microtubule-based establishment of cell polarity. In chapter 6 we develop a minimal model for spontaneous and persistent polarity in a spherical cell. The model is based on the ability of microtubules to bind and transport proteins. We show analytically that our system supports a stable unipolar symmetry-broken state for a wide range of parameters. We validate the predictions of the model by $2 \mathrm{D}$ particle-based simulations. Our model may form the basis for creating polarity in a minimal cell-like environment using a biochemical reconstitution approach.

In chapter 7 we focus on the polarization of an elliptical cell. We apply stochastic simulations in order to find possible predominant directions of microtubule ordering. We study the competition between geometry dependent effects which promote bipolar ordering (described in Chapters 3 and 4) and the polarization mechanism (described in Chapter 6) that promotes unipolar ordering. Except of the simulations, the results are also confirmed theoretically by regarding a model with a pre-established bias. 
Chapter 1. Introduction 


\section{Part I}

\section{Branched Nucleation}





\section{2 \\ The effect of anisotropic microtubule-bound nucleations on ordering in the plant cortical array}

The microtubules play a key role in the elongation of plant cells, as they define the direction along which cell expands. Microtubules achieve this by forming inside the plasma membrane a structure known as cortical array. The nucleation of the cortical microtubules is carried out by nucleation complexes that are mostly attached to pre-existing microtubules. This attachment combined with collisional interactions among microtubules can result in a spontaneous alignment of microtubules, which has been shown by stochastic simulations. In this chapter, we proceed to the theoretical foundation of this phenomenon, which is caused by the known as 'branched' nucleation. Furthermore, we reveal the existence of a single parameter that determines the relative influence between the microtubule-bound nucleation and the background isotropic one. 


\subsection{Introduction}

Microtubules are dynamic filamentous protein aggregates and a key constituent of the cytoskeleton of all eukaryotic cells. They can reach lengths of several ten's of $\mu \mathrm{m}$ 's and are therefore able to span lengths comparable to the dimensions of cells. This allows them to perform a host of functions related to establishing and maintaining the morphology and mechanical properties of cells. In plants, a number of cell types, e.g. the well-studied root cells, grow by expansion along a single axis [31, and microtubules play a key role in defining this axis. They do so by setting up a plant-unique structure called the transverse cortical array, a highly aligned arrangement of microtubules bound to the inside of the plasma membrane [16]. There is mounting evidence that the microtubules in the transverse array guide the insertion and the direction of motion cellulose synthase complexes [32, 33]. These complexes deposit cellulose microfibrils, the main architectural component of the plant cell wall. The widely accepted idea is that the ensuing transverse orientation of the cellulose microfibrils allows the cells to elongate in a single direction, whilst maintaining mechanical integrity in the face of an appreciable internal osmotic pressure (turgor).

A crucial aspect of microtubules and the structures they form is that they are both dynamic and intrinsically out of thermodynamic equilibrium. Individual microtubules alternate stochastically between growing and shrinking states in an energy-consuming process dubbed "dynamic instability" [34]. In the plant cortical array, where the microtubules are bound to the plasma membrane and hence form an effectively $2 \mathrm{D}$ system, it is this process which drives collisions between growing microtubules and obstructing microtubules. The stochastic and angle-of-incidence dependent outcomes of these collisions are classified as (i) zippering, where the incoming microtubule alters its course by bending and continues to grow along the obstructing microtubule, (ii) induced catastrophes, where the incoming microtubule rapidly switches from a growing to a shrinking state, and (iii) cross-overs, where the incoming microtubule manages to "slip over" the obstructing one and continues to grow in its original direction [17]. Several groups have shown how the ordering of the cortical array (hereafter abbreviated as CA) can be understood on the basis of these collisions, using both computer simulations [35, 18, 36] and an analytical model [37]. The dynamical instability process implies that microtubules can shrink to zero 
length and thus are expected to have a finite lifetime. This means that in order to achieve a steady state, new microtubules need to be nucleated at a finite rate, for which cells employ specific nucleation complexes called $\gamma$-TuRCS [38]. In most of the modeling approaches the nucleation of new microtubules was assumed to occur homogeneously distributed over the 2D "cortex" and in arbitrary directions, as is observed during the initial stages of CA formation. In the later stages of CA formation, when there is an appreciable density of microtubules, most of the nucleations actually occur from preexisting microtubules, and moreover with a specific orientational distribution with respect to the direction of the parent microtubule [14, 15, 39].

In a previous paper [21] we have addressed the impact of this microtubulebound orientationally-biased nucleation mechanism on CA ordering using computer simulations, showing among others that (partially) co-aligning the newly nucleated microtubules with the parent microtubules provides a positive feedback on the ordering process, significantly widening the range of parameters for which the aligned state is stable. Here we provide the theoretical underpinning of these observations by generalizing our previously developed analytical model to explicitly incorporate anisotropic microtubule-bound nucleations. This allows us to perform a full parametric analysis of the location of the ordering transition in the presence of these more complex nucleations. Significantly, this analysis reveals that the coalignment parameter $\nu_{2}$ introduced on phenomenological grounds in [21], is indeed also formally the system parameter that most strongly influences the propensity of the system to order. Moreover, the full model allows us to go beyond the simulations by also considering the effect of different firing rates of the nucleation complexes whether in the bound- or the unbound state, and showing how this difference interacts with the effects of differential binding of the nucleation complexes to the microtubules.

The outline of the paper is as follows. In Section 2.2 we present our theory, first recapping the basic elements of the formalism (Section 2.2.1), then implementing a binding equilibrium for the nucleation complexes (Section 2.2.2 , discuss the treatment of anisotropic nucleations (Section 2.2.3), finishing with a dimensional analysis, which reveals a single parameter that governs the influence of the microtubule-bound nucleations (Section 2.2.4). In Section 2.3 we locate the critical values of the control parameter of the system using bifurcation analysis (Section 2.3.1), calculate the latter nu- 
merically (Section 2.3.2) and compare with simulations (Section 2.3.3). We end with conclusions and outlook in Section 2.4.

\subsection{Theoretical framework}

\subsubsection{Basic theory}

Here we first recap the basic elements of the analytical model by Hawkins, Tindemans and Mulder [37] for the mechanism of orientational order of the plant CA.

The cortical microtubules are confined to a $2 \mathrm{D}$ plane and each microtubule is considered to consist of one or more straight segments with a fixed orientation, connected end-to-end. The intrinsic dynamics of the microtubules is described by the standard two-state dynamic instability model of Dogterom and Leibler [9], which assumes that each microtubule has a "plus" end on its final segment that is either growing with speed $v^{+}$ or shrinking with speed $v^{-}$. This plus end can switch stochastically from growing to shrinking, a so-called spontaneous catastrophe, with rate $r_{c}$, or from shrinking to growing, a so-called rescue, with rate $r_{r}$. The dynamics of the interactions between colliding microtubules dynamics is encoded into the probabilities $P_{c}(\theta)$ of observing an induced catastrophe, $P_{z}(\theta)$ of a zippering event and $P_{x}(\theta)$ of a crossover when the collision occurs at a relative angle $\theta$. An individual segment of a microtubule can be either in the growing $(+)$ or shrinking $(-)$ state, provided it is the final segment, and otherwise is in the inactive state (0). The main ingredients of the model are graphically summarized in figure 2.1.

A coarse-grained description is employed for the alignment of microtubules in the cortical array: Instead of individual microtubules, local densities of microtubule segments are considered. The system is assumed to be spatially homogeneous and has as fundamental variables the areal number densities $m_{i}^{\sigma}(l, \theta, t)$ of segments in state $\sigma \in\{0,+,-\}$, where the segment number $i$ keeps track of the number of orientation-changing zippering events $(=i-1)$ that preceded in creating the segments, $l$ is the length of the segments and $\theta$ their orientation and $t$ the time. These densities obey a set of partial differential evolution equations determined by the overall rates of growth and shrinkage, spontaneous and induced catastrophes, zippering and reactivation of inactive segments through shrinking. 


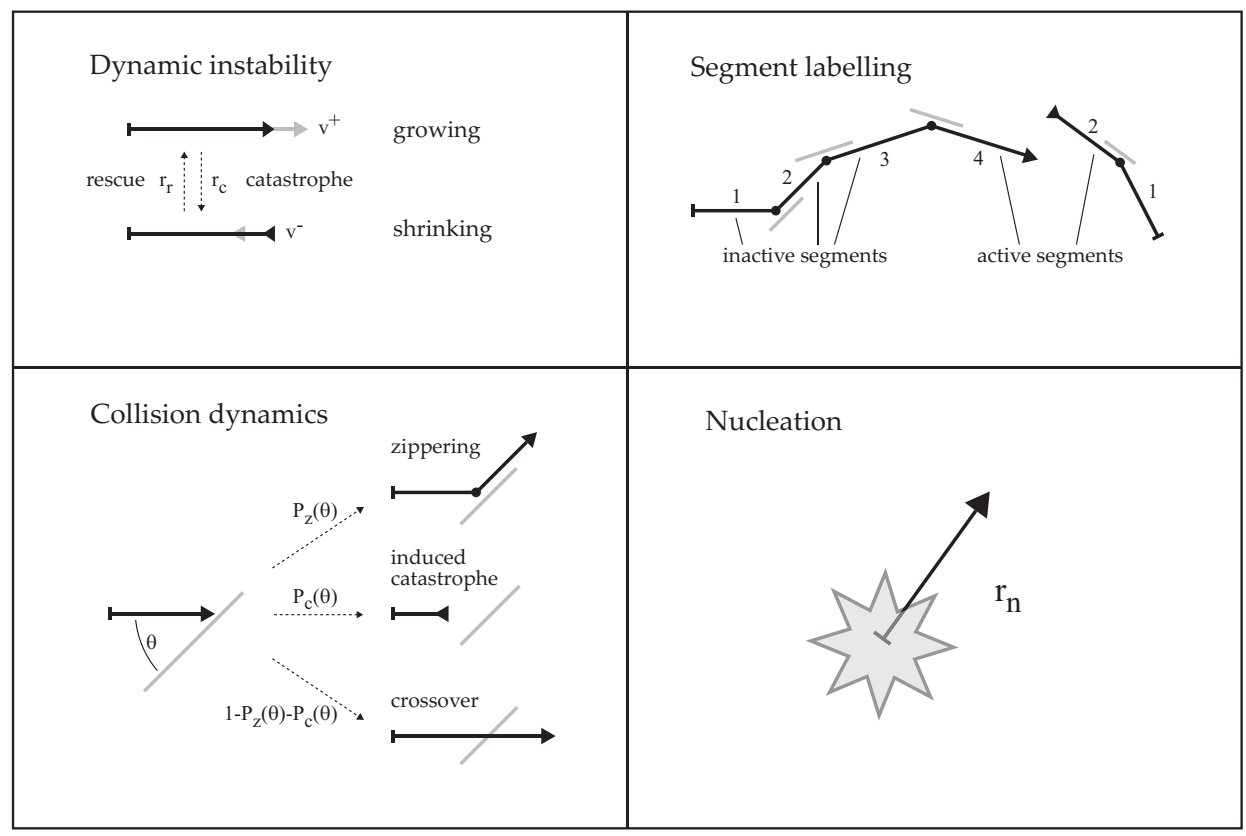

Figure 2.1: Schematic illustration of the main ingredients of our model for cortical microtubule dynamics. Top left: The dynamical instability mechanism, with random switches between a growing and a shrinking state. Top right: Labelling of microtubule segments from the nucleation point (bar),increasing after each zippering event (collision with gray obstructing microtubule). Bottom left: Collision outcomes. Bottom right: Nucleation of new microtubules.

In steady state, the length of all segments, independently of type, turns out to be distributed exponentially with a common average segment length $l(\theta)$

$$
m_{i}^{\sigma}(l, \theta)=m_{i}^{\sigma}(\theta) e^{-l / l(\theta)},
$$

which introduces the angle dependent density at zero length $m_{i}^{\sigma}(\theta)$. The stationarity of the total length of microtubules implies the balance equation

$$
v^{+} m_{i}^{+}(l, \theta)=v^{-} m_{i}^{-}(l, \theta)
$$

An important role is played by the total length density $k(\theta)$ of all micro- 
tubule segments in direction $\theta$, which is defined as

$$
k(\theta)=\sum_{i=1}^{\infty} \int d l l\left[m_{i}^{+}(l, \theta)+m_{i}^{-}(l, \theta)+m_{i}^{0}(l, \theta)\right] .
$$

Next, the quantities $Q_{i}(\theta)$, are defined as the ratio between the densities of inactive and active microtubules for segments with index $i$

$$
Q_{i}(\theta)=\frac{m_{i}^{0}(\theta)}{m_{i}^{+}(\theta)+m_{i}^{-}(\theta)} .
$$

As is shown in [37, these quantities do not in fact depend on the index $i$, hence $Q_{i}(\theta)=Q(\theta)$. Finally, the quantity $t(\theta)$ is defined as the overall density of active segments

$$
\begin{aligned}
t(\theta) & =\sum_{i=1}^{\infty} \int_{0}^{\infty} d l\left[m_{i}^{+}(l, \theta)+m_{i}^{-}(l, \theta)\right] \\
& =l(\theta) \sum_{i=1}^{\infty}\left[m_{i}^{+}(\theta)+m_{i}^{-}(\theta)\right],
\end{aligned}
$$

where the second equality is a result of the exponential distribution equation Eq. (2.1).

We now formulate the governing equations of the system. The "crosssection" of the collisions is determined in part by the geometrical factor $\sin \left(\theta-\theta^{\prime}\right)$, where $\theta$ is the angle of the incoming growing microtubule segment and $\theta^{\prime}$ is the angle of the microtubule "scatterer" with respect to a fixed reference frame. This factor is absorbed into the interaction probabilities by defining

$$
f\left(\theta-\theta^{\prime}\right) \equiv\left|\sin \left(\theta-\theta^{\prime}\right)\right| P_{f}\left(\theta-\theta^{\prime}\right)
$$

where $f \in\{c, z, x\}$, with $c$ denoting induced catastrophes, $z$ zippering events and $x$ crossovers. We will assume that the collisions are insensitive to the relative orientation of the microtubule plus-minus end polarities, so that $f(\theta)=f(\pi-\theta)$ for $f \in\{c, z, x\}$. The average segment length $l(\theta)$ is shown to satisfy

$$
\frac{1}{l(\theta)}=-g+\int d \theta^{\prime}\left[c\left(\theta-\theta^{\prime}\right)+z\left(\theta-\theta^{\prime}\right)\right] k\left(\theta^{\prime}\right)
$$


where

$$
g=\frac{r_{r}}{v^{-}}-\frac{r_{c}}{v^{+}}
$$

is called the growth parameter, and characterizes the behavior of the non interacting system. Here we will limit ourselves to the case $g<0$, which ensures a priori that the length of the microtubules remains intrinsically bounded. While the second term on the right-hand side of Eq. (2.6) could in principle offset the effect of $g>0$, the resulting system is expected to be absolutely unstable with respect to alignment [37] which would lead to a degenerate non-steady state with continuously elongating microtubules. Although the dynamical parameters measured in some plant systems (see e.g. [35]) would suggest that $g$ may be positive, the situation in planta is complicated by the activity of the microtubule severing protein Katanin (see e.g. [40]), which provides an additional channel for microtubule length control. The effects of the latter, as well as other factors such length capping due to the presence of cell boundaries, are beyond the scope of the present work. For a more extended discussion of these issues please refer to [41].

Using the Eqs. 2.1 through 2.5 we find that the length density $k(\theta)$ obeys

$$
k(\theta)=l(\theta)[1+Q(\theta)] t(\theta)
$$

The inactive/active ratio $Q(\theta)$ satisfies the following self-consistency equation

$$
Q(\theta)=\int d \theta^{\prime} z\left(\theta-\theta^{\prime}\right) k\left(\theta^{\prime}\right) l\left(\theta^{\prime}\right)\left(1+Q\left(\theta^{\prime}\right)\right) .
$$

Finally, the overall density of active tip segments $t(\theta)$ obeys

$$
t(\theta)=\left(1+\frac{v^{+}}{v^{-}}\right) l(\theta) m_{1}^{+}(\theta)+l(\theta) k(\theta) \int d \theta^{\prime} z\left(\theta-\theta^{\prime}\right) t\left(\theta^{\prime}\right) .
$$

We see that the density $m_{1}^{+}(\theta)$ of zero-length segments that have not been created by a zippering event only appears explicitly in Eq. (2.9) for the density of active tips. This density is determined by the rate and the orientation at which new microtubules are nucleated, which serves as a boundary condition to the steady state equations

$$
v^{+} m_{1}^{+}(\theta)=r_{n}(\theta) \text {. }
$$

The precise form of the angle-dependent nucleation rate $r_{n}(\theta)$ per unit area, which hitherto was considered to be an isotropic constant, will be discussed below. 


\subsubsection{Binding equilibrium of the nucleation complexes}

While in the initial stages of cortical array formation almost all of the microtubule nucleations occur on random locations in the cortex and in random directions, it appears that with increasing array density most of the nucleations are localized to existing microtubules [15, 39]. Moreover, these microtubule-bound nucleations occur with a distinct distribution of orientations with respect to the parent microtubule. The majority of all these nucleations is due to the presence of specific nucleation complexes, so-called $\gamma$-TuRCs, that are able to bind specifically to microtubules, with the precise geometry of the individual binding configurations giving rise to the observed angular pattern of the microtubule-bound nucleations. To include these effects into our model, we first of all assume that there is finite density of available nucleation complexes. Next, we assume that binding and unbinding happens on a time scale fast compared to the other processes. These assumptions allow us to model the fraction of bound- versus unbound nucleation complexes as being determined by a binding equilibrium.

We let nucleation complexes bind to a unit of length of microtubule per unit of system area with a rate $r_{b}$. In steady state, the length density of microtubules is given by Eq. 2.3

$$
k_{t o t}=\int_{0}^{2 \pi} d \theta k(\theta)
$$

Bound nucleation complexes can unbind from their parent microtubule with rate $r_{u}$. The overall (areal) density of nucleation complexes is given by $n_{\text {tot }}=n_{b}+n_{u}$, where $n_{b}$ and $n_{u}$ are the bound- and unbound densities respectively. With these definitions, the chemical equilibrium condition then reads

$$
n_{b} r_{u}=r_{b} k_{t o t} n_{u}
$$

Thus, the fractions of bound and unbound nucleation complexes are given by

$$
\begin{aligned}
& x_{b}=\frac{n_{b}}{n_{t o t}}=\frac{r_{b} k_{t o t}}{r_{b} k_{t o t}+r_{u}} \equiv \frac{k_{t o t}}{k_{t o t}+k_{\frac{1}{2}}} \\
& x_{u}=\frac{n_{u}}{n_{t o t}}=\frac{r_{u}}{r_{b} k_{t o t}+r_{u}} \equiv \frac{k_{\frac{1}{2}}}{k_{t o t}+k_{\frac{1}{2}}},
\end{aligned}
$$


where the cross-over density $k_{\frac{1}{2}}=\frac{r_{u}}{r_{b}}$, which equals the microtubule length density for which exactly half the nucleation complexes are bound, controls the shift between the regime of microtubule densities dominated by unbound nucleations and bound ones respectively.

\subsubsection{Anisotropic distribution of nucleation angles}

To determine the angle-dependent nucleation rate $r_{n}(\theta)$ we now differentiate between nucleations occurring from unbound nucleation complexes and bound ones. Consistent with observations, we take the nucleations from the unbound complexes to be isotropically distributed and assume that an available unbound complex "fires" with a rate $r_{n}^{u}$. The distribution of nucleation angles in the global reference frame associated with unbound nucleations is trivially given by

$$
\psi_{u}(\theta)=\frac{1}{2 \pi} .
$$

Nucleations from microtubule-bound complexes, on the other hand, have been shown to occur with a distinct orientational pattern with respect to the parent microtubule. We describe this pattern through the normalized relative nucleation angle distribution $\nu(\Delta \theta)$, with

$$
\int_{0}^{2 \pi} d \Delta \theta \nu(\Delta \theta)=1
$$

To determine the distribution of nucleation angles due to the bound nucleations in the global reference frame, this distribution must be convolved with the orientation distribution of the microtubules themselves, thus

$$
\psi_{b}(\theta)=\frac{1}{k_{t o t}} \int_{0}^{2 \pi} d \theta^{\prime} \nu\left(\theta-\theta^{\prime}\right) k\left(\theta^{\prime}\right),
$$

which as one checks is again normalised. Finally, we take the firing rate of bound nucleation complexes to be $r_{n}^{b}$. In the following, the only condition we will assume on the relative nucleation angle distribution is that it is mirror symmetric with respect to the parent-microtubule axis, i.e. $\nu(\Delta \theta)=$ $\nu(-\Delta \theta)$.

With these ingredients we can now construct the overall angle-dependent nucleation rate

$$
r_{n}(\theta)=n_{u} r_{n}^{u} \psi_{u}(\theta)+n_{b} r_{n}^{b} \psi_{b}(\theta),
$$


where $n_{u}$ and $n_{b}$ are the densities of unbound and bound nucleation complexes respectively, as derived in the previous section. We graphically illustrate the elements in the construction of the angle-dependent nucleation rate in figure 2.2 .

\section{Nucleation modes}

unbound bound
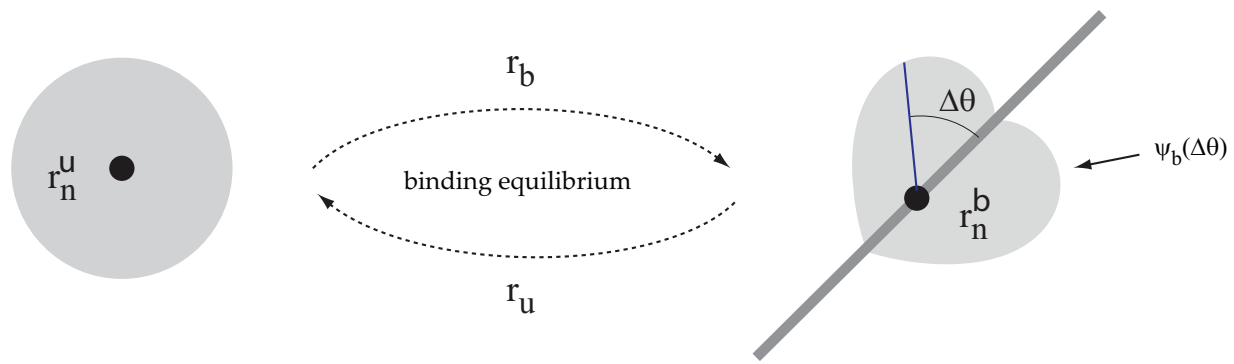

isotropic

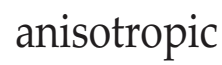

Figure 2.2: Schematic illustration of the model for the overall angledependent nucleation rate, consisting of a chemical equilibrium between isotropic nucleation from unbound nucleation complexes (left) and anisotropic nucleation from microtubule-bound nucleation complexes (right). The light gray shape surrounding the nucleation complex (solid circle) represents a polar plot of the distribution of relative nucleation angles, circular (= isotropic) in the unbound case (left) and a nontrivial function $\psi_{b}(\Delta \theta)$ in the bound case (right).

It is now convenient to introduce the overall nucleation rate

$$
r_{n}=n_{b} r_{n}^{b}+n_{u} r_{n}^{u}
$$

and the relative firing rate

$$
\rho=\frac{r_{n}^{b}}{r_{n}^{u}}
$$

and to define

$$
r_{n}(\theta)=\frac{r_{n}}{2 \pi} R(\theta)
$$


which introduces the absolute nucleation angle distribution $R(\theta)$. The latter is explicitly given by

$$
R(\theta)=\frac{\rho k_{t o t}}{\rho k_{t o t}+k_{\frac{1}{2}}} 2 \pi \psi_{b}(\theta)+\frac{k_{\frac{1}{2}}}{\rho k_{t o t}+k_{\frac{1}{2}}}
$$

where we have used the results of Eq. 2.13). The choice of the normalization

$$
\frac{1}{2 \pi} \int_{0}^{2 \pi} d \theta R(\theta)=1
$$

for this distribution serves to minimize the number of explicit factors $2 \pi$ appearing in the final set of dimensionless equations discussed below.

\subsubsection{Dimensional analysis}

The set of Eqs. 2.6 - 2.9 can be simplified by the use of dimensional analysis. The adoption of the length scale

$$
l_{0}=\left(\frac{1}{\pi} \frac{v^{+}}{\left(1+\frac{v^{+}}{v^{-}}\right) r_{n} /(2 \pi)}\right)^{1 / 3}
$$

allows the definition of the dimensionless variables

$$
\begin{aligned}
L(\theta) & =l(\theta) / l_{0}, \\
K(\theta) & =\pi k(\theta) l_{0}, \\
T(\theta) & =\pi l_{0}^{2} t(\theta),
\end{aligned}
$$

and dimensionless control parameter of the problem

$$
G=g l_{0}=\left[\frac{2 v^{+} v^{-}}{r_{n}\left(v^{+}+v^{-}\right)}\right]^{1 / 3}\left(\frac{r_{r}}{v^{-}}-\frac{r_{c}}{v^{+}}\right)
$$

It is convenient to adopt the collision operators

$$
\mathbf{C}[h](\theta)=\frac{1}{\pi} \int_{0}^{2 \pi} d \theta^{\prime} c\left(\theta-\theta^{\prime}\right) h\left(\theta^{\prime}\right), \mathbf{Z}[h](\theta)=\frac{1}{\pi} \int_{0}^{2 \pi} d \theta^{\prime} z\left(\theta-\theta^{\prime}\right) h\left(\theta^{\prime}\right)
$$


where $h(\theta)$ is any integrable function. With these definitions we can formulate the set of dimensionless equations describing the system in the presence of anisotropic nucleation

$$
\begin{aligned}
\frac{1}{L(\theta)} & =-G+\mathbf{C}[K](\theta)+\mathbf{Z}[K](\theta) \\
K(\theta) & =L(\theta)(1+Q(\theta)) T(\theta) \\
Q(\theta) & =\mathbf{Z}[L K(1+Q)](\theta) \\
T(\theta) & =L(\theta) R(\theta)+L(\theta) K(\theta) \mathbf{Z}[T](\theta)
\end{aligned}
$$

The expression for the absolute nucleation angle distribution is facilitated by the introduction of two additional operators

$$
\begin{aligned}
& \mathbf{V}[h](\theta)=\frac{1}{\pi} \int_{0}^{2 \pi} d \theta^{\prime} \nu\left(\theta-\theta^{\prime}\right) h\left(\theta^{\prime}\right) \\
& \mathbf{U}[h](\theta)=\frac{1}{\pi} \int_{0}^{2 \pi} d \theta^{\prime} h\left(\theta^{\prime}\right)
\end{aligned}
$$

Non-dimensionalizing the microtubule length density using the definition in Eq. 2.24, we find

$$
R(\theta)=\frac{2 \pi \beta \mathbf{V}[K]+1}{\beta \mathbf{U}[K]+1}
$$

where the parameter $\beta$ is given by

$$
\beta=\frac{\rho}{k_{\frac{1}{2}} l_{0}} .
$$

Strikingly, this single parameter suffices to capture the relative importance of the bound nucleations with respect to the unbound ones: it is high whenever the affinity of the nucleation complexes to the microtubules is high ( $\operatorname{small} k_{\frac{1}{2}}$ ) or when the nucleation rate of the bound complexes is high (large $\rho$ ) compared to that of the unbound ones. 


\section{$2.3 \quad$ Results}

\subsubsection{Bifurcation analysis}

In the isotropic state of the system, all angular dependence drops out and the set of equations becomes

$$
\begin{aligned}
\frac{1}{\bar{L}} & =-G+\left(\hat{c}_{0}+\hat{z}_{0}\right) \bar{K} \\
\bar{K} & =\bar{L}(1+\bar{Q}) \bar{T} \\
\bar{Q} & =\hat{z}_{0} \bar{L} \bar{K}(1+\bar{Q}) \\
\bar{T} & =\bar{L} \bar{R}+\hat{z}_{0} \bar{L} \bar{K} \bar{T}
\end{aligned}
$$

where throughout the overbar denotes quantities of the isotropic phase. Here, as in the following, the coefficients $\hat{c}_{n}$ and $\hat{z}_{n}$ are the eigenvalues of the operators $\mathbf{C}$ and $\mathbf{Z}$ respectively, on the basis of the $\operatorname{cosines} \cos (n \theta)$

$$
\mathbf{C}[\cos (n \theta)]=\hat{c}_{n} \cos (n \theta), \quad \mathbf{Z}[\cos (n \theta)]=\hat{z}_{n} \cos (n \theta) .
$$

Next, equation 2.33 readily gives

$$
\bar{R}=1
$$

showing that the equations for the isotropic state are, as expected, independent of the angular details of the nucleation mechanism and therefore the same as those considered in [37] (cf. Eqs. (40) in that reference).

Using straightforward elimination we can derive the equations

$$
\bar{K}\left(\hat{c}_{0} \bar{K}-G\right)^{2}=1
$$

and

$$
\bar{N}\left(1-\hat{z}_{0} \bar{N}\right)^{2} G^{3}-\left[\left(\hat{c}_{0}+\hat{z}_{0}\right) \bar{N}-1\right]^{3}=0
$$

where $\bar{N}=\bar{L} \bar{K}$, that yield to equivalent ways of characterizing the isotropic state as a function of the control parameter $G$.

In order to perform a bifurcation analysis, probing the stability of the isotropic state against anisotropic perturbations, we parametrize the solutions to the full problem as

$$
\begin{aligned}
L & =\bar{L}(1+\lambda) \\
K & =\bar{K}(1+\kappa) \\
Q & =\bar{Q}(1+\chi) \\
T & =\bar{T}(1+\tau)
\end{aligned}
$$


where we assume that the perturbations $\lambda, \kappa, \chi$ and $\tau$ are small. Inserting into the equations (2.27), (2.28), (2.29) and (2.30) and expanding to the first order in the perturbations we obtain

$$
\begin{aligned}
-\lambda & =\bar{N}(\mathbf{C}[\kappa]+\mathbf{Z}[\kappa]) \\
\kappa & =\lambda+\tau+\hat{z}_{0} \bar{N} \chi \\
\chi & =\frac{1}{\hat{z}_{0}} \mathbf{Z}\left[\kappa+\lambda+\hat{z}_{0} \bar{N} \chi\right] \\
\tau & =\lambda+\bar{N}\left(\hat{z}_{0} \kappa+\mathbf{Z}[\tau]\right)+\left(1-\hat{z}_{0} \bar{N}\right) \mathbf{B}[\kappa]
\end{aligned}
$$

where

$$
\mathbf{B}[\kappa]=\frac{\beta \bar{K}}{2 \beta \bar{K}+1}(2 \pi \mathbf{V}[\kappa]-\mathbf{U}[\kappa])
$$

Eliminating $\lambda, \chi$ and $\tau$ from these equations we find a linear eigenvalue problem for the length density perturbation $\kappa$

$$
\left(1-\hat{z}_{0} \bar{N}\right) \kappa=-2 \bar{N} \mathbf{C}[\kappa]+\left(1-\hat{z}_{0} \bar{N}\right) \mathbf{B}[\kappa]
$$

which is satisfied whenever $\kappa(\theta)$ is an eigenfunction of both the operators $\mathbf{C}$ and $\mathbf{B}$. Given the symmetries of the operators $\mathbf{C}, \mathbf{V}$ and $\mathbf{U}$ the relevant set of common eigenfunctions is the family $\cos (2 j \theta)$, where $j \geq 1(j=0$ is not an anisotropic perturbation). We assume, as in [37, that the longest wavelength perturbation $\cos (2 \theta)$, i.e. the case $j=1$, corresponds to the first break of symmetry on increasing $G$. Inserting this assumption into the eigenvalue equation 2.52 , we get the equation which implicitly defines the location of the corresponding bifurcation point

$$
\left(1-\hat{z}_{0} \bar{N}\right)=-2 \bar{N} \hat{c}_{2}+\left(1-\hat{z}_{0} \bar{N}\right) \frac{2 \beta \bar{K}}{2 \beta \bar{K}+1} \nu_{2}
$$

Here we have introduced the co-alignment parameter

$$
\nu_{2}=\int_{0}^{2 \pi} d \theta \cos (2 \theta) \nu(\theta) \in[-1,1],
$$

which provides the relevant measure for the degree of orientational coalignment of microtubules originating from bound nucleation events with their parent microtubules. Its appearance in the bifurcation equation 2.53 provides an a posteriori theoretical underpinning for its heuristic use in 
the analysis of the simulation data in [21]. When $\nu_{2}=1$, which occurs e.g. when $\nu(\theta)=\delta(\theta)$ ), the nucleated microtubules are perfectly co-aligned (either in the plus- or minus end direction) with the parent microtubule, when $\nu_{2}=-1$, which occurs when $\nu(\theta)=\frac{1}{2}\left(\delta\left(\theta-\frac{\pi}{2}\right)+\delta\left(\theta+\frac{\pi}{2}\right)\right)$, the nucleated microtubules are maximally dis-aligned $(=$ perpendicular $)$ to the parent microtubule. Finally, the intermediate case $\nu_{2}=0$ occurs when the microtubules are either nucleated evenly into the "neutral" directions $\pm \frac{\pi}{4}$ and $\pm \frac{3 \pi}{4}$ or simply isotropically $\left(\nu(\theta)=\frac{1}{2 \pi}\right)$.

The bifurcation equation $(2.53)$, together with the isotropic state equations (2.41) and (2.42), allow us to calculate the critical value of the control parameter $G_{*}$ at which the bifurcation occurs. We start by rewriting Eq. 2.53 as

$$
\bar{N}=\frac{1}{\hat{z}_{0}+\bar{M}}
$$

where

$$
\bar{M}=\left(-2 \hat{c}_{2}\right) \frac{(2 \beta \bar{K}+1)}{\left(2 \beta \bar{K}\left(1-\nu_{2}\right)+1\right)} .
$$

Inserting this form of $\bar{N}$ into Eq. 2.42, we obtain

$$
G_{*}=\bar{M}^{1 / 3}\left(\frac{\hat{c}_{0}}{\bar{M}}-1\right) .
$$

We note that this result is valid independent of the sign of $G_{*}$. However, in line with our choice, discussed in Sect. 2.2.1, to limit the analysis to the regime $G<0$, so that we are ensured an ordered steady-state can exist, we now enquire under which constraints in fact $G_{*}<0$. As by definition $\hat{c}_{0}>0$, we should need both $\bar{M}>0$ as well as $\bar{M}>\hat{c}_{0}$. We now note that $\operatorname{sgn}(\bar{M})=-\operatorname{sgn}\left(\hat{c}_{2}\right)$, so that we require $\hat{c}_{2}<0$. As discussed in [37], this is generically the case for induced catastrophe probabilities $P_{c}(\theta)$ that are (semi)monotonically increasing in $\theta$ on the interval $\left[0, \frac{\pi}{2}\right]$, which in turn is consistent with the in vivo observations [17]. Next, we note that for $\nu_{2} \in[0,1]$, i.e. the range of nucleations that are effectively in the forward to neutral directions with respect to the parent microtubules, $\bar{M} \geq\left(-2 \hat{c}_{2}\right)$, so that it is then sufficient to require, as in [37], that $\left(-2 \hat{c}_{2}\right)>$ $\hat{c}_{0}$. The latter requirement is readily met for realistic induced catastrophe probability profiles. Finally, the available data indicates that $\nu_{2} \sim 0.7-0.9$, i.e. in the required regime [15, 39]. In the following we therefore freely adopt these constraints. 
We also note that in the limit $\beta=0$, where the nucleation complexes do not bind to the microtubules and all nucleations take place isotropically in the bulk, Eq. 2.57) correctly reduces to

$$
G_{*}^{(0)}=\left(-2 \hat{c}_{2}\right)^{1 / 3}\left(\frac{\hat{c}_{0}}{-2 \hat{c}_{2}}-1\right),
$$

the result previously obtained in [37]. After some algebra, we can also obtain the first order effect of a non-zero $\nu_{2}$ on the location of the transition

$$
G_{*}^{(1)}=\left.\frac{\partial G_{*}}{\partial \nu_{2}}\right|_{\nu_{2}=0}=\frac{1}{3 \hat{c}_{2}}\left(-2 \hat{c}_{2}\right)^{\frac{1}{3}}\left(\hat{c}_{0}-\hat{c}_{2}\right) \frac{2 \beta \bar{K}_{*}^{(0)}}{\left(2 \beta \bar{K}_{*}^{(0)}+1\right)}<0
$$

where $\bar{K}_{*}^{(0)}$ is the critical length density at $\nu_{2}=0$. This result indicates that for positive co-alignment $\left(\nu_{2}>0\right)$ the bifurcation point is shifted towards lower $G$ values, indicating a widening of the ordered region. Note also that Eq. (2.57) implies that, in spite of the anisotropic nucleation mechanism, the location of the transition does not depend on the presence or absence of the zippering mechanism, in line with the analysis presented in [37.

To obtain the bifurcation point for arbitrary values of $\nu_{2}$, we introduce (2.57) into the equation 2.41) coupling $\bar{K}$ and $G$ in the isotropic state, ultimately obtaining a 15 th order polynomial equation in $\sqrt{\bar{K}}$ (not shown). Numerical solutions of this equation allow us to identify the unique positive real root that yields the critical value of the microtubule length density $\bar{K}_{*}$, which in turn can be used in Eq. (2.41) to back out the critical value $G_{*}$ of the control parameter. The results of this procedure are discussed in the next section.

\subsubsection{Numerical solutions}

To present our numerical results on the location of the ordering transition we first have to choose a set of collision probabilities. Following Refs. 35] and [36] we opted for the following stylized representation of the available data

$$
\begin{aligned}
& P_{z}(\Delta \theta)= \begin{cases}1 & \Delta \theta<\theta_{c}=40^{\circ} \\
0 & \theta_{c} \leq \Delta \theta \leq 90^{\circ}\end{cases} \\
& P_{c}(\Delta \theta)=\left\{\begin{array}{cc}
0 & \Delta \theta<\theta_{c}=40^{\circ} \\
p_{c} & \theta_{c} \leq \Delta \theta \leq 90^{\circ}
\end{array}\right.
\end{aligned}
$$


In figure 2.3 we show the result for the critical value $G_{*}$ as a function of $\nu_{2}$ for a few values of the parameter $\beta$ in the specific case $p_{c}=0.5$.

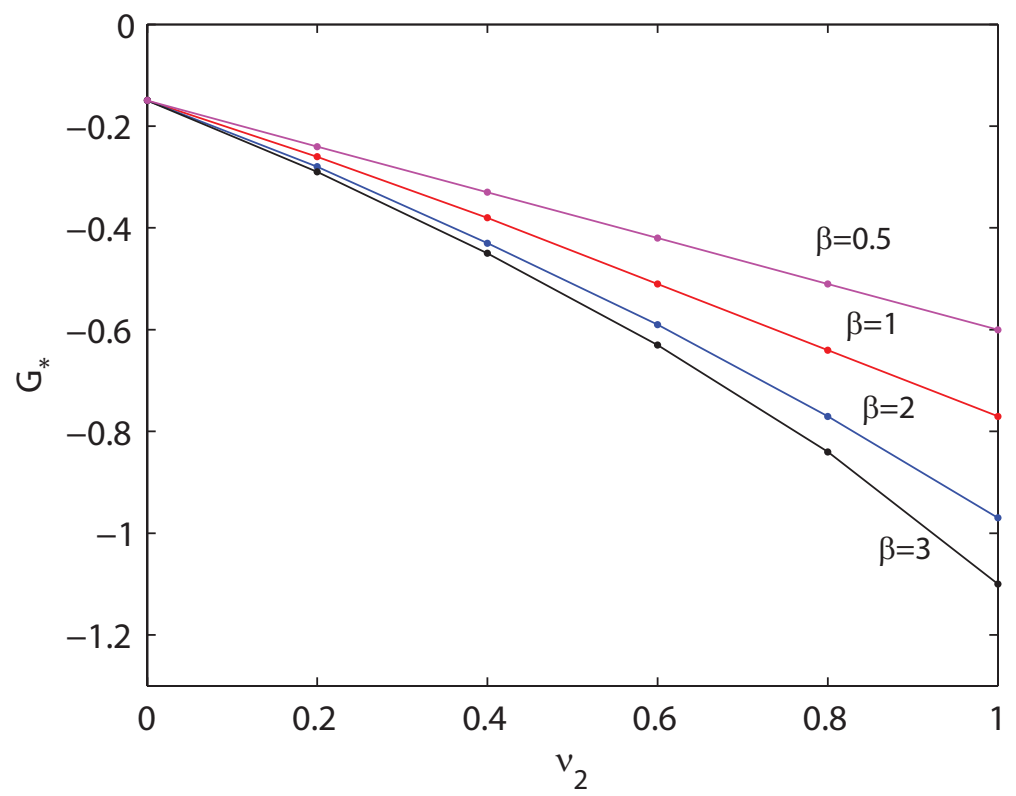

Figure 2.3: Critical values $G_{*}$ of the control parameter $G$ as a function of the degree of co-aligned nucleation $\nu_{2}$ for 4 different values of the parameter $\beta$.

We see that for all values of the parameter $\beta$, which governs the strength of the anisotropic nucleation mechanism, the critical value of the control parameter is a decreasing function of the coalignment parameter $\nu_{2}$. This means that the regime of microtubule dynamical parameter for which the system establishes an ordered state is widened. Indeed, we can interpret the co-aligned nucleation of new microtubules as an additional positive feedback mechanism on the basic "survival of the aligned" mechanism presented in [37]: longer-lived aligned microtubules also generate "offspring" that is similarly aligned, will therefore experience fewer catastrophe inducing collisions, and hence "inherit" the longevity of their parents. 


\subsubsection{Comparison to simulations}

In order to verify that the results of our mean-field theory are reasonable, we here compare them with results from particle-based simulations. First we compare with the semi-realistic simulations described in [21] (see figure 2.4). These employed a microtubule-bound nucleation distribution with $n \%$ of nucleation along the parent microtubule in the forward direction, and the remainder isotropically distributed. By varying the percentage $n$, the degree of co-alignment, which in this case is simply given by $\nu_{2}=n / 100$, can be varied over the full range $[0,1]$. A second-rank order parameter $S_{2}$ is defined as

$$
S_{2}=\frac{\left|\int_{0}^{2 \pi} d \theta e^{i 2 \theta} K(\theta)\right|}{\int_{0}^{2 \pi} d \theta K(\theta)}
$$

and is calculated from the simulation data for different values of the control parameter $G$ [21]. Then, the critical control parameter $G_{*}$ is easily calculated.

In spite of the fact that the simulation employed the so-called simple bundle collision dynamics, in which an microtubule impinging on a bundle only sees a single target microtubule [42], and had explicitly treadmilling microtubules [43, the qualitative agreement between the predicted locations of the transitions and the ones observed in the simulations is satisfactory (see figure 2.4), specifically in reproducing the marked widening of the ordered region.

To fully probe the validity of the theory, especially regarding the prediction that the single parameter $\beta$ governs the strength of the anisotropic nucleation effects (at constant $\nu_{2}$ ), we vary independently the parameters $\rho$ and $k_{1 / 2}$ (see Eq. (2.34)) as we have adapted the simulations to accommodate for a different nucleation rate of the bound- versus the unbound nucleations.

As a small technical aside, we remark that our event-driven simulation scheme intrinsically employs a constant propensity $r_{\operatorname{try}}$ for nucleation events. To differentiate the rates of bound- versus unbound nucleations $(\rho \neq 1)$ we have used a rejection method discarding a fraction of bound (in the case $\rho<1$ ) or unbound (in the case $\rho>1$ ) nucleations. This implies that overall nucleation rate is less than the fixed propensity and moreover state dependent (through the density dependence of the microtubulebinding equilibrium of the nucleation complexes cf. Eq. (2.13)). To com- 


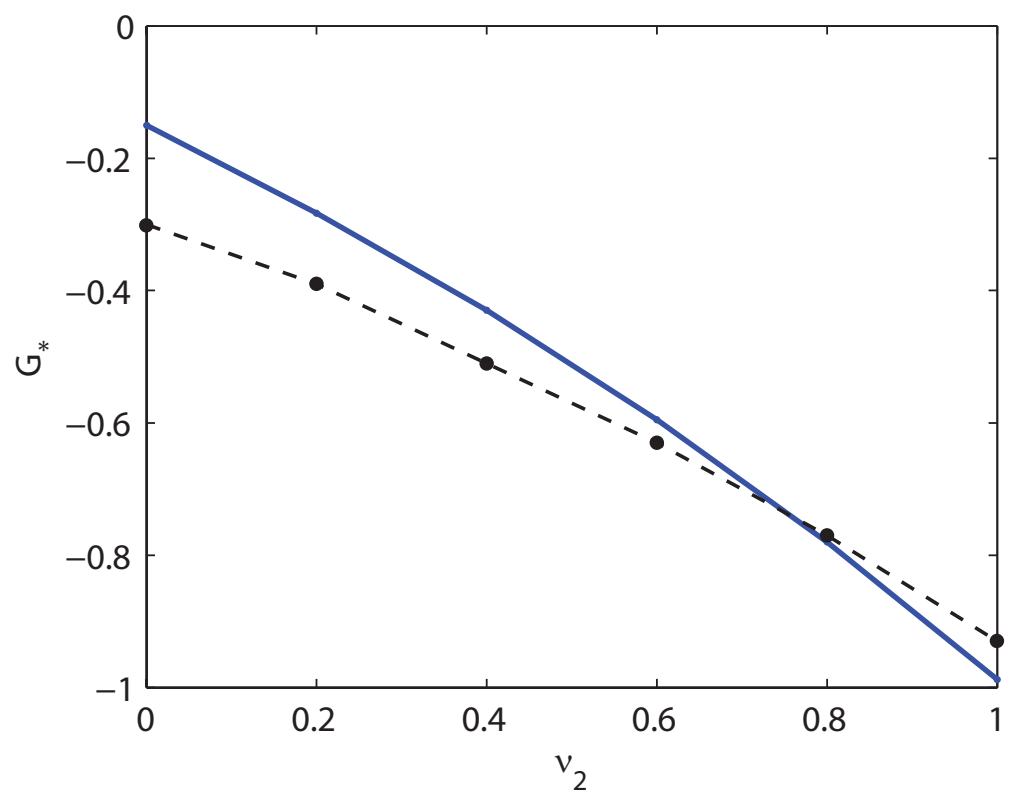

Figure 2.4: Comparison between simulation results [21] (filled circles, dashed line to guide the eye) and theoretical predictions (solid line) for the critical values $G_{*}$ of the control parameter as a function of the degree of co-aligned nucleation $\nu_{2}$ for a model with $p_{c}=0.5$.

pare the theory, which assumes a constant overall nucleation rate $r_{n}$, with the results from the simulation we performed a scaling between the simulation value of the coupling parameter $G_{s i m}\left(r_{\operatorname{try} n}\right)$ and the theoretical value $G \equiv G_{t h}\left(r_{n}\right)$, given by

$$
G_{t h}\left(r_{n}\right)= \begin{cases}\left(\left\langle x_{b}\right\rangle+\frac{1}{\rho}\left\langle x_{u}\right\rangle\right)^{-\frac{1}{3}} G_{\text {sim }}\left(r_{\text {try } n}\right) & \rho \geq 1 \\ \left(\rho\left\langle x_{b}\right\rangle+\left\langle x_{u}\right\rangle\right)^{-\frac{1}{3}} G_{\text {sim }}\left(r_{\text {try } n}\right) & \rho<1\end{cases}
$$

where $\left\langle x_{u}\right\rangle$ (and $\left\langle x_{b}\right\rangle=1-\left\langle x_{u}\right\rangle$ ) are averaged values determined from the simulations.

We first check that indeed the critical value $G_{*}$ of the control parameter $\mathrm{G}$ depends only on $\beta$, by choosing a fixed product $\rho \cdot k_{1 / 2}=\beta \cdot l_{0}$ and running a number of simulations with ranging value of $\rho$. The simulation results 
show (see figure 2.5) that the transition point to order is common for all the tested pairs $\left(\rho, k_{1 / 2}\right)$ which correspond to the same value of $\beta$.

The results for the critical value of the coupling parameter as a function of the nucleation co-alignment parameter $\nu_{2}$ for a number of different values of $\beta$, shown in figure 2.6, prove that the theory is able to quantitatively reproduce the simulation data.

As a separate check, to explicitly rule out the influence of zippering, we have also performed the simulations with $P_{z}\left(\Delta \theta<\theta_{c}\right)=1$, i.e. with the same zippering/induced catastrophe strength ratio as in the standard collision model Eq. 2.60), which yielded no significant differences. Indeed, as we can see in figure 2.7, the presence of zippering does not affect the location of the transition point of the system and its route to order.

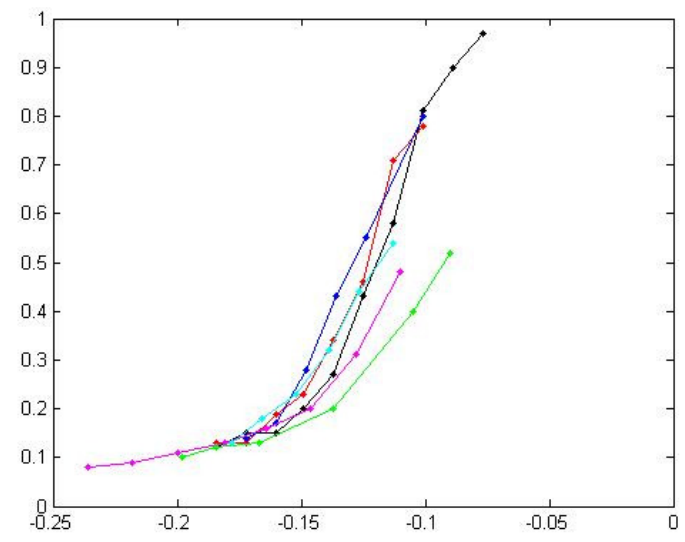

Figure 2.5: Order parameter $S_{2}$ as a function of the scaled to theory control parameter $G$ for different pairs $\left(\rho, k_{1 / 2}\right)$ with ratio $\rho / k_{1 / 2}=\beta l_{0}=0.75$ and $\nu_{2}=0.60$. Blue: $(1,4 / 3)$, red: $(1.5,2.0)$, black: $(3,4)$, cyan: $(2 / 3,8 / 9)$ and pink: $(6,8)$. 


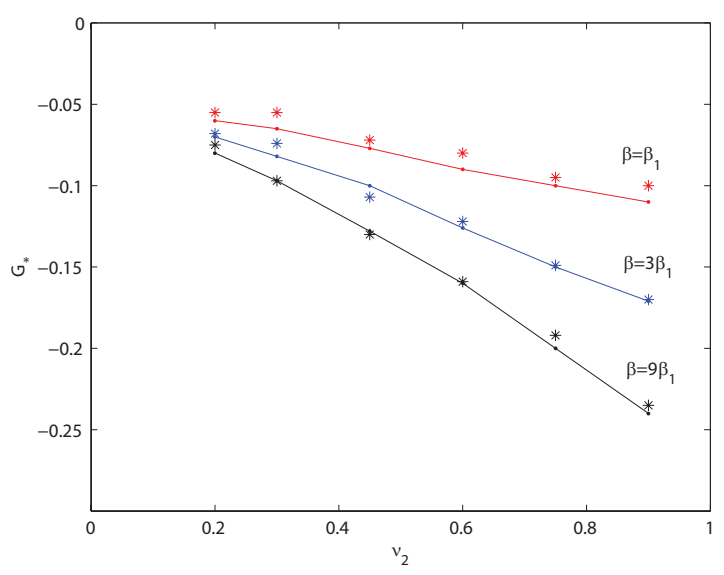

Figure 2.6: Critical values $G_{*}$ of the control parameter as a function of the degree of co-aligned nucleation $\nu_{2}$ for a model with $p_{\text {cat }}=0.01$ and $p_{z i p}=$ 0 for 3 different values of $\beta$, where $\beta_{1}=0.018$ (Underlying parameters: $\left.k_{\frac{1}{2}}=4 \mu m^{-1}, \rho=1, l_{0}=4.7425 \mu m\right)$. Stars: simulation results. Lines: theoretical results.

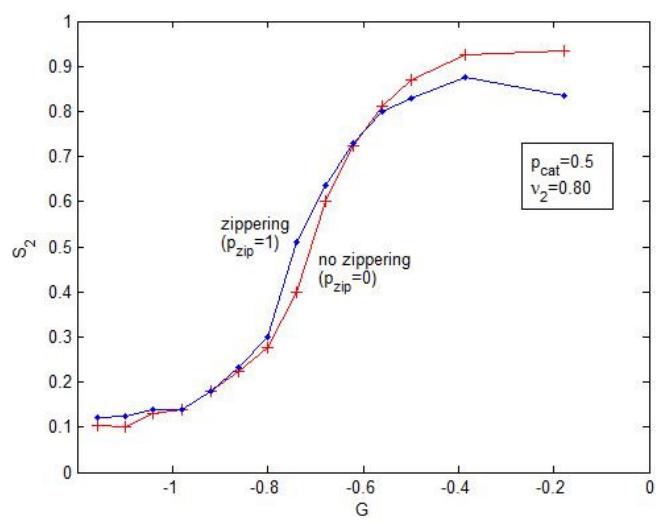

Figure 2.7: Order parameter $S_{2}$ as a function of $G$ with and without zippering interactions. The distribution parameter $\nu_{2}$ of nucleation is equal to 0.80 for both cases. The two systems are equilibrated for 20,000 seconds before the alignment is measured. Results are averaged over 60 runs. 


\subsection{Conclusion}

We have shown that the theoretical framework for describing the selforganization of the microtubule cortical cytoskeleton in plant cells first presented in [37] can be robustly extended to include the biologically relevant microtubule-bound anisotropic nucleations. The relative importance of this effect as compared to the background isotropic nucleation events is predicted to depend on a single dimensionless number $\beta$, that takes into account both the affinity of nucleation complexes for microtubules, as well as a possible binding-state dependence of their firing rate. Our bifurcation analysis furthermore reveals that averaged co-alignment of the bound nucleations with the parent microtubule, captured by the parameter $\nu_{2}$, is the main determinant of the location of the transition. The significant widening of the ordered regime with increasing $\nu_{2}$ can be ascribed to a positive feedback mechanism that enhances the "survival of the aligned" mechanism already described in [18]: aligned microtubules "beget" co-aligned "offspring" thus increasing the survival of the preferential direction. Finally, the comparisons with the particle-based simulations, show that the theory, albeit of a mean-field nature, is a robust approximation to full dynamical system including the spatial dependencies.

The work described here is a first step in the extension of the model to include a number of factors that are known to be involved in the in vivo ordering process. Here, we specifically mention the effects of minus-end treadmilling, microtubule severing by Katanin-like proteins and finiteness of the available tubulin monomer pool. Including these effects is part of our ongoing research effort. 
Part II

\section{Microtubule-based phenomena induced by shape}





\title{
3 \\ Cell shape and the spatial distribution of microtubules
}

\begin{abstract}
We address the question how cell shape can modulate the intrinsic dynamics of microtubules leading to anisotropic spatial distributions. To that end we develop a 3D analytical model for microtubules that can stall at a cell boundary and from which they detach at a variable rate. The resulting equations are solved for a model cell with the shape of a general ellipsoid and microtubules emanating from a single centrosome located in the center of the cell. Depending on the parameters, either a longitudinal or an transverse ordering pattern is observed. These analytical results are subsequently verified by $2 \mathrm{D}$ stochastic simulations. Finally, we extend our analysis to non-centrosomal spatially dispersed nucleation sites.
\end{abstract}




\subsection{Introduction}

Recently evidence was presented for a microtubule organization mechanism that is driven by the cell shape in epidermal cells of Drosophila [44. This raises the general question of how the fact that microtubules live within the finite boundaries of the cell influences their spatial distribution.

There are a number of factors that should play a role. First and foremost is the intrinsic dynamics of the microtubules. The values of the dynamical parameters that characterize this dynamics control the mean length of the microtubules, and hence the degree to which they are sensitive to the finite size of the cell. Note that these dynamical parameters themselves may be subject to cellular control through the interactions of microtubules with microtubule associated proteins (MAPs) [45], which, among others, have the ability to affect their stability, and hence their length distribution. Next, an important role is played by how and where they are nucleated. In proliferative animal cells microtubules are commonly nucleated from a single localized cellular structure called a centrosome [46]. However, in most differentiated cells microtubules are nucleated from non-centrosomal sites and form arrays that are specific depending on the cell type [47. Non-centrosomal microtubule organizing centers (ncMTOCs) are e.g. found in human fibroblasts, neurons, plant epidermal cells, myotubes, intestinal epithelial cells, and in C. elegans germ cells [48]. Last but not least, there are the interactions of microtubules with the cell boundaries. It has been shown experimentally in vivo [49] and in vitro [22] that a microtubule can stall to the cell boundary. So, depending on the details of their interactions with the boundary, microtubules can have a variable residence time there, which can in principle be both longer or shorter than the typical waiting time to a catastrophe for a free microtubule. Another effect known to play a role is the fact that growing microtubules can exert forces causing them to buckle [50] or even to slide along the boundary. Together these factors will determine to what extent the spatial distribution of the microtubules is dependent on the geometry of the cell.

Here, we wish to explore these effects from fundamental point of view. In this chapter we will focus on the simplest setting, in which the mechanical effects of the interaction with the boundary, like buckling and sliding, are neglected. We will return to the effects of polymerization force driven sliding in the next chapter. We first consider on the organization of centro- 
somal microtubules. To that end, we extend to 3D a previously developed 1D model for microtubules interacting with a boundary [51], and derive explicit equations for the angle-dependent microtubule length distributions in the steady state. This formalism is then applied to a model cell shaped as a general ellipsoid with a single centrosome at its center. Most real cells are axially symmetric and the latter geometry can be reasonably well approximated by a $2 \mathrm{D}$ ellipse with the aspect ratio as the single shape parameter. In this case we can also directly compare the analytical results with computationally less demanding $2 \mathrm{D}$ stochastic simulations, which verify the mean-field assumptions implicitly in the theory. Then, extend our study to the non-centrosomal microtubules, first by considering the effects of a non-central nucleation site and finally a spatially homogeneous distribution of nucleation sites, both within an ellipse-shaped cell.

\subsection{Model for microtubules nucleated isotropically in a bounded space}

We consider a point-like model "centrosome" located at a position $\mathbf{r}$ within a bounded volume. It is homogeneously covered by nucleation sites that can nucleate microtubules in a radial direction from this location. We describe these nucleation sites through a constant (solid) angular density $m$, so that the maximum number of microtubules that can emanate from the centrosome is given by $M=4 \pi m$. Each nucleation site can be labelled by the unit vector $\hat{\omega}$ of the direction in which it can nucleate a microtubule. If it is unoccupied, it will do so at a rate $r_{n}$. It is convenient to consider an occupied nucleation site as a dormant microtubule, waiting to be nucleated. Once a microtubule has been nucleated, it follows the standard dynamical instability model [9], with growth speed $v_{+}$, shrinking speed $v_{-}$catastrophe rate $r_{+}$and rescue rate $r_{-}$. When the microtubule hits the cell boundary it stalls, remaining there until it switches to the shrinking state with a rate $r_{u}$. The length of the microtubule when it hits the boundary is given by $L(\hat{\omega} \mid \mathbf{r})$, with the latter function encoding all the relevant information about the shape of the cell. The dependent variables in our model are respectively

- $m_{0}(\hat{\omega}, t \mid \mathbf{r})$ : The density (per solid angle) of dormant microtubules pointing in the direction $\hat{\omega}$ at time $t$.

- $m_{i}^{+}(l, \hat{\omega}, t \mid \mathbf{r})$ : The density (per solid angle per unit length) of growing 


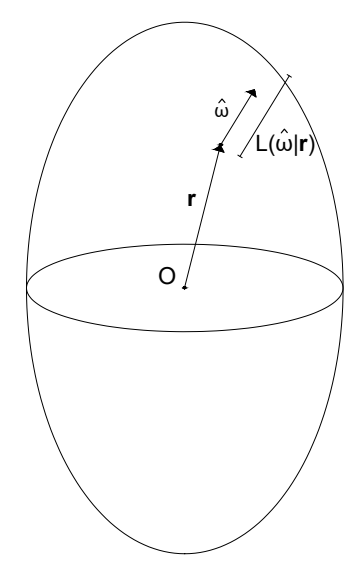

Figure 3.1: Microtubule nucleation from position $\mathbf{r}$ inside a bounded geometry. We can see the unit vector $\hat{\omega}$ and the distance $L(\hat{\omega} \mid \mathbf{r})$ to the boundary at the direction of nucleation.

microtubules of length $l$.

- $m_{i}^{-}(l, \hat{\omega}, t \mid \mathbf{r})$ : The density (per solid angle per unit length) of shrinking microtubules of length $l$.

- $m_{b}(\hat{\omega}, t \mid \mathbf{r})$ : The density (per solid angle) of microtubules bound to the surface.

In terms of these variables, the dynamic equations of the system read

$$
\begin{aligned}
\partial_{t} m_{i}^{+}(l, \hat{\omega}, t \mid \mathbf{r}) & =-v_{+} \partial_{l} m_{i}^{+}(l, \hat{\omega}, t \mid \mathbf{r})+r_{-} m_{i}^{-}(l, \hat{\omega}, t \mid \mathbf{r})-r_{+} m_{i}^{+}(l, \hat{\omega}, t \mid \mathbf{r}) \\
\partial_{t} m_{i}^{-}(l, \hat{\omega}, t \mid \mathbf{r}) & =v_{-} \partial_{l} m_{i}^{-}(l, \hat{\omega}, t \mid \mathbf{r})-r_{-} m_{i}^{-}(l, \hat{\omega}, t \mid \mathbf{r})+r_{+} m_{i}^{+}(l, \hat{\omega}, t \mid \mathbf{r}) \\
\partial_{t} m_{0}(\hat{\omega}, t \mid \mathbf{r}) & =-r_{n} m_{0}(\hat{\omega}, t \mid \mathbf{r})+v_{-} m_{i}^{-}(l=0, \hat{\omega}, t \mid \mathbf{r}) \\
\partial_{t} m_{b}(\hat{\omega}, t \mid \mathbf{r}) & =-r_{u} m_{b}(\hat{\omega}, t \mid \mathbf{r})+v_{+} m_{i}^{+}(L(\hat{\omega} \mid \mathbf{r}), \hat{\omega}, t \mid \mathbf{r})
\end{aligned}
$$


with boundary conditions

$$
\begin{aligned}
& r_{n} m_{0}(\hat{\omega}, t \mid \mathbf{r})=v_{+} m_{i}^{+}(l=0, \hat{\omega}, t \mid \mathbf{r}) \\
& r_{u} m_{b}(\hat{\omega}, t \mid \mathbf{r})=v_{-} m_{i}^{-}(L(\hat{\omega} \mid \mathbf{r}), \hat{\omega}, t \mid \mathbf{r})
\end{aligned}
$$

We introduce the integrated quantities

$$
\begin{aligned}
& m_{i}^{+}(\hat{\omega}, t \mid \mathbf{r})=\int_{0}^{L(\hat{\omega} \mid \mathbf{r})} d l m_{i}^{+}(l, \hat{\omega}, t \mid \mathbf{r}) \\
& m_{i}^{-}(\hat{\omega}, t \mid \mathbf{r})=\int_{0}^{L(\hat{\omega} \mid \mathbf{r})} d l m_{i}^{-}(l, \hat{\omega}, t \mid \mathbf{r}) \\
& m_{i}^{a}(\hat{\omega}, t \mid \mathbf{r})=m_{i}^{+}(\hat{\omega}, t \mid \mathbf{r})+m_{i}^{-}(\hat{\omega}, t \mid \mathbf{r})
\end{aligned}
$$

respectively the total number of growing, shrinking and active microtubules in the given direction in the interior. Integrating Eqs. (3.1), (3.2) over the relevant lengths, and adding the resulting equations with (3.3), (3.4) and using the boundary conditions, we find

$$
\partial_{t} m_{i}^{+}(\hat{\omega} \mid \mathbf{r}, t)+\partial_{t} m_{i}^{-}(\hat{\omega} \mid \mathbf{r}, t)+\partial_{t} m_{0}(\hat{\omega} \mid \mathbf{r}, t)+\partial_{t} m_{b}(\hat{\omega} \mid \mathbf{r}, t)=0
$$

verifying the conservation of total number of microtubules in our systems, which we can also express as

$$
m_{0}(\hat{\omega}, t \mid \mathbf{r})+m_{i}^{a}(\hat{\omega}, t \mid \mathbf{r})+m_{b}(\hat{\omega}, t \mid \mathbf{r})=m
$$

In the steady state Eqs. 3.1), 3.2), 3.3), 3.4 are written

$$
\begin{aligned}
v_{+} \partial_{l} m_{i}^{+}(l, \hat{\omega} \mid \mathbf{r}) & =r_{-} m_{i}^{-}(l, \hat{\omega} \mid \mathbf{r})-r_{+} m_{i}^{+}(l, \hat{\omega} \mid \mathbf{r}) \\
-v_{-} \partial_{l} m_{i}^{-}(l, \hat{\omega} \mid \mathbf{r}) & =-r_{-} m_{i}^{-}(l, \hat{\omega} \mid \mathbf{r})+r_{+} m_{i}^{+}(l, \hat{\omega} \mid \mathbf{r}) \\
v_{+} m_{i}^{+}(l=0, \hat{\omega} \mid \mathbf{r}) & =r_{n} m_{0}(\hat{\omega} \mid \mathbf{r}) \\
v_{+} m_{i}^{+}(L(\hat{\omega} \mid \mathbf{r}), \hat{\omega} \mid \mathbf{r}) & =r_{u} m_{b}(\hat{\omega} \mid \mathbf{r})
\end{aligned}
$$

Adding Eqs. 3.12 and 3.13 gives

$$
\partial_{l}\left\{v_{+} m_{i}^{+}(l, \hat{\omega} \mid \mathbf{r})-v_{-} m_{i}^{-}(l, \hat{\omega} \mid \mathbf{r})\right\}=0
$$

Combining Eqs. (3.15) and (3.6), yields

$$
v_{+} m_{i}^{+}(L(\hat{\omega} \mid \mathbf{r}), \hat{\omega} \mid \mathbf{r})=v_{-} m_{i}^{-}(L(\hat{\omega} \mid \mathbf{r}), \hat{\omega} \mid \mathbf{r})
$$


which shows that the constant of integration of the previous equation vanishes and, so, we get

$$
v_{+} m_{i}^{+}(l, \hat{\omega} \mid \mathbf{r})=v_{-} m_{i}^{-}(l, \hat{\omega} \mid \mathbf{r})
$$

This allows us to eliminate $m^{-}(l, \hat{\omega} \mid \mathbf{r})$ in Eq. 3.12$)$ and solve this equation using Eq. (3.14)

$$
m_{i}^{+}(l, \hat{\omega} \mid \mathbf{r})=\frac{r_{n}}{v_{+}} m_{0}(\hat{\omega} \mid \mathbf{r}) e^{-l / \bar{l}}
$$

where

$$
\bar{l}=\left(\frac{r_{+}}{v_{+}}-\frac{r_{-}}{v_{-}}\right)^{-1}
$$

is the mean length of unperturbed microtubules. Similarly, we get

$$
m_{i}^{-}(l, \hat{\omega} \mid \mathbf{r})=\frac{r_{n}}{v_{-}} m_{0}(\hat{\omega} \mid \mathbf{r}) e^{-l / \bar{l}}
$$

We can now use the boundary condition $(3.6)$ to find

$$
m_{b}(\hat{\omega} \mid \mathbf{r})=\frac{r_{n}}{r_{u}} m_{0}(\hat{\omega} \mid \mathbf{r}) e^{-L(\hat{\omega} \mid \mathbf{r}) / \bar{l}}
$$

The final unknown, $m_{0}(\hat{\omega} \mid \mathbf{r})$, can now be obtained, by employing the conservation law (cf. Eq. (3.10))

$$
m_{0}(\hat{\omega} \mid \mathbf{r})+\int_{0}^{L(\hat{\omega} \mid \mathbf{r})} d l\left\{m_{i}^{+}(l, \hat{\omega} \mid \mathbf{r})+m_{i}^{-}(l, \hat{\omega} \mid \mathbf{r})\right\}+m_{b}(\hat{\omega} \mid \mathbf{r})=m
$$

Inserting the equations for $m_{i}^{+}(l, \hat{\omega} \mid \mathbf{r}), m_{i}^{-}(l, \hat{\omega} \mid \mathbf{r})$ and $m_{b}(\hat{\omega} \mid \mathbf{r})$ we obtain

$$
m_{0}(\hat{\omega} \mid \mathbf{r})\left\{1+r_{n}\left(\frac{1}{v_{+}}+\frac{1}{v_{-}}\right) \bar{l}\left(1-e^{-L(\hat{\omega} \mid \mathbf{r}) / \bar{l}}\right)+\frac{r_{n}}{r_{u}} e^{-L(\hat{\omega} \mid \mathbf{r}) / \bar{l}}\right\}=m
$$

or

$$
m_{0}(\hat{\omega} \mid \mathbf{r})=m \frac{t_{0}}{t_{0}+\bar{t}\left(1-F_{b}(\hat{\omega} \mid \mathbf{r})\right)+t_{b} F_{b}(\hat{\omega} \mid \mathbf{r})}
$$


Here, we have introduced

$$
\begin{aligned}
t_{0} & =\frac{1}{r_{n}} \\
\bar{t} & =\left(\frac{1}{v_{+}}+\frac{1}{v_{-}}\right) \bar{l} \\
t_{b} & =\frac{1}{r_{u}} \\
F_{b}(\hat{\omega} \mid \mathbf{r}) & =e^{-L(\hat{\omega} \mid \mathbf{r}) / \bar{l}}
\end{aligned}
$$

with $t_{0}$ the mean life time in the dormant state, $\bar{t}$ the mean lifetime of a microtubule without a boundary constraint, $t_{b}$ the mean lifetime of the bound state and $F_{b}(\hat{\omega} \mid \mathbf{r})$ loosely interpreted as the fraction of active microtubules that enters the bound state. This representation allows an easy interpretation of the steady-state densities in terms of the fraction of the time spent in the specific state. In this way, we consider the density $m_{\alpha}(\hat{\omega})$ of the active microtubules

$$
m_{i}^{a}(\hat{\omega} \mid \mathbf{r})=\int_{0}^{L(\hat{\omega} \mid \mathbf{r})} d l\left\{m_{i}^{+}(l, \hat{\omega} \mid \mathbf{r})+m_{i}^{-}(l, \hat{\omega} \mid \mathbf{r})\right\}
$$

which, by combining (3.19), 3.21 and 3.25, becomes

$$
m_{\alpha}(\hat{\omega} \mid \mathbf{r})=m \frac{\bar{t}\left(1-F_{b}(\hat{\omega} \mid \mathbf{r})\right)}{t_{0}+\bar{t}\left(1-F_{b}(\hat{\omega} \mid \mathbf{r})\right)+t_{b} F_{b}(\hat{\omega} \mid \mathbf{r})}
$$

Similarly, combining equations 3.22 and 3.25 we get

$$
m_{b}(\hat{\omega} \mid \mathbf{r})=m \frac{t_{b} F_{b}(\hat{\omega} \mid \mathbf{r})}{t_{0}+\bar{t}\left(1-F_{b}(\hat{\omega} \mid \mathbf{r})\right)+t_{b} F_{b}(\hat{\omega} \mid \mathbf{r})}
$$

While the number densities of microtubules in a certain direction are interesting, a physically more relevant description of the spatial distribution of the microtubules is provided by the length densities, which measure e.g. the capacity to bias motor-driven transport in a given direction, which relies both on the ability of motor-proteins to bind, itself proportional to length, 
as well as the distance of which transport can place. We therefore We regard now the length densities $l_{i}^{a}(\hat{\omega} \mid \mathbf{r}), l_{b}(\hat{\omega} \mid \mathbf{r})$ of the active and the bound microtubules in the steady state

$$
\begin{aligned}
& l_{i}^{a}(\hat{\omega} \mid \mathbf{r})=\int_{0}^{L(\hat{\omega} \mid \mathbf{r})} d l l m_{i}^{a}(l, \hat{\omega} \mid \mathbf{r}) \\
& l_{b}(\hat{\omega} \mid \mathbf{r})=L(\hat{\omega} \mid \mathbf{r}) m_{i}^{b}(\hat{\omega} \mid \mathbf{r})
\end{aligned}
$$

Using Eqs. 3.19), 3.21), 3.25) and (3.33), we find

$$
l_{a}(\hat{\omega} \mid \mathbf{r})=m \frac{\bar{t}}{t_{0}+\bar{t}\left(1-F_{b}(\hat{\omega} \mid \mathbf{r})\right)+t_{b} F_{b}(\hat{\omega} \mid \mathbf{r})}\left(\bar{l}-(L(\hat{\omega} \mid \mathbf{r})+\bar{l}) F_{b}(\hat{\omega} \mid \mathbf{r})\right)
$$

And, using also Eqs. (3.22) and (3.34), we get

$$
l_{b}(\hat{\omega} \mid \mathbf{r})=m \frac{t_{b}}{t_{0}+\bar{t}\left(1-F_{b}(\hat{\omega} \mid \mathbf{r})\right)+t_{b} F_{b}(\hat{\omega} \mid \mathbf{r})} L(\hat{\omega} \mid \mathbf{r}) F_{b}(\hat{\omega} \mid \mathbf{r})
$$

Finally, the total length density $l(\hat{\omega} \mid \mathbf{r})$ is defined as

$$
l(\hat{\omega} \mid \mathbf{r})=l_{a}(\hat{\omega} \mid \mathbf{r})+l_{b}(\hat{\omega} \mid \mathbf{r})
$$

For estimating the degree of microtubule alignment, we need to consider a nematic order parameter. To this end, we regard the unit vector along the microtubules given by $\hat{\omega}=(\sin \theta \cos \phi, \sin \theta \sin \phi, \cos \theta)$. Then the $3 \mathrm{D}$ order parameter tensor is given by

$$
\mathbf{Q}=\left\langle\frac{1}{2}(3 \hat{\omega} \otimes \hat{\omega}-\mathbb{I})\right\rangle
$$

which works out as

$\mathbf{Q}=\left\langle\left(\begin{array}{ccc}\frac{3}{2} \cos ^{2}(\phi) \sin ^{2}(\theta)-\frac{1}{2} & \frac{3}{2} \cos (\phi) \sin ^{2}(\theta) \sin (\phi) & \frac{3}{2} \cos (\theta) \cos (\phi) \sin (\theta) \\ \frac{3}{2} \cos (\phi) \sin ^{2}(\theta) \sin (\phi) & \frac{3}{2} \sin ^{2}(\theta) \sin ^{2}(\phi)-\frac{1}{2} & \frac{3}{2} \cos (\theta) \sin (\theta) \sin (\phi) \\ \frac{3}{2} \cos (\theta) \cos (\phi) \sin (\theta) & \frac{3}{2} \cos (\theta) \sin (\theta) \sin (\phi) & \frac{3}{2} \cos ^{2}(\theta)-\frac{1}{2}\end{array}\right)\right\rangle$

The random element $Q_{i j}$ of $\mathbf{Q}$ for e.g. the length density $l_{a}(\hat{\omega} \mid \mathbf{r})$ of the active microtubules is

$$
Q_{i j}=\frac{\int_{0}^{\pi} d \theta \sin \theta \int_{0}^{2 \pi} d \phi \frac{1}{2}(3 \hat{\omega} \otimes \hat{\omega}-\mathbb{I})_{i j} l_{a}(\hat{\omega} \mid \mathbf{r})}{\int_{0}^{\pi} d \theta \sin \theta \int_{0}^{2 \pi} d \phi l_{a}(\hat{\omega} \mid \mathbf{r})}
$$


The eigenvalues of $\mathbf{Q}$ are the scalar order parameters at the directions defined by the three components of the unit vector $\hat{\omega}$.

The $2 \mathrm{D}$ uniaxial order parameter tensor is defined by

$$
\mathbf{S}=\langle 2 \hat{\omega} \otimes \hat{\omega}-\mathbb{I}\rangle
$$

with $\hat{\omega}=(\cos \theta, \sin \theta)$ the unit vector along the microtubules. We can easily get

$$
\mathbf{S}=\left\langle\left(\begin{array}{cc}
\cos 2 \theta & \sin 2 \theta \\
\sin 2 \theta & -\cos 2 \theta
\end{array}\right)\right\rangle
$$

Then, for e.g. the case of the length density $l_{a}(\hat{\omega} \mid \mathbf{r})$ it holds

$$
\langle\cos 2 \theta\rangle=\frac{\int_{0}^{2 \pi} d \theta \cos 2 \theta l_{\alpha}(\hat{\omega} \mid \mathbf{r})}{\int_{0}^{2 \pi} d \theta l_{\alpha}(\hat{\omega} \mid \mathbf{r})}
$$

and

$$
\langle\sin 2 \theta\rangle=\frac{\int_{0}^{2 \pi} d \theta \sin 2 \theta l_{\alpha}(\hat{\omega} \mid \mathbf{r})}{\int_{0}^{2 \pi} d \theta l_{\alpha}(\hat{\omega} \mid \mathbf{r})}
$$

The positive eigenvalue of the $2 \mathrm{D}$ uniaxial order parameter tensor is

$$
S=\sqrt{\langle\cos 2 \theta\rangle^{2}+\langle\sin 2 \theta\rangle^{2}}
$$

which is the scalar order parameter. For $S=0$ we have a perfectly isotropic system, while for $S=1$ all microtubules align to the same direction.

In order to distinguish the longitudinal bipolar state from the transverse one, we use the order parameter $S_{2}$, which measures the degree of order along the longitudinal axis

$$
S_{2}=\langle\cos 2 \theta\rangle
$$

For $S_{2}>0$ we get a longitudinal ordering, whereas for $S_{2}<0$ we get a transverse one.

For the unipolar state we use the order parameter

$$
S_{1}=\sqrt{\langle\cos \theta\rangle^{2}+\langle\sin \theta\rangle^{2}}
$$




\section{$3.3 \quad$ Results}

\subsubsection{General ellipsoidal cell with a central centrosome}

We now consider as a model cell shape a general ellipsoid with a centrosome fixed at its center $\mathbf{r}=0$. For notational convenience we will no longer explicitly refer to the position of the centrosome. Using a Cartesian reference frame with the origin at the centrosome, the ellipsoid is described by the equation

$$
\frac{x^{2}}{a^{2}}+\frac{y^{2}}{b^{2}}+\frac{z^{2}}{c^{2}}=1
$$

Then, by symmetry the $\mathbf{Q}$ order parameter tensor reduces to the diagonal form

$$
\mathbf{Q}=\left(\begin{array}{ccc}
\left\langle\frac{3}{2} \cos ^{2}(\phi) \sin ^{2}(\theta)-\frac{1}{2}\right\rangle & 0 & 0 \\
0 & \left\langle\frac{3}{2} \sin ^{2}(\theta) \sin ^{2}(\phi)-\frac{1}{2}\right\rangle & 0 \\
0 & 0 & \left\langle\frac{3}{2} \cos ^{2}(\theta)-\frac{1}{2}\right\rangle
\end{array}\right)
$$

The three diagonal elements are in this case the eigenvalues of the $3 \mathrm{D}$ order parameter and, so, are the scalar order parameters in the directions of the ellipsoid axes $a, b, c$.

The distance $L(\theta, \phi)$ from the centrosome to the boundary in terms of the polar angle $\phi$ and azimuthal angle $\theta$ is given by

$$
L(\theta, \phi)=\frac{a b c}{\sqrt{a^{2} b^{2}+c^{2}\left(a^{2}-b^{2}\right) \sin ^{2} \theta \sin ^{2} \phi+b^{2}\left(c^{2}-a^{2}\right) \sin ^{2} \theta}}
$$

Two example cell shapes, a prolate and an oblate ellipsoid, are shown in figure 3.2 .

By keeping the two axes lengths $a$ and $c$ constant and varying between them the length of the third axis $b$, we get different ellipsoids with characteristics between a prolate and an oblate ellipsoid. For each of them and for the set of parameters shown in Table (3.1), we calculate the three scalar order parameters that give the degrees of microtubule length ordering parallel to axes $a, b, c$. The results are shown in figure 3.3 . 

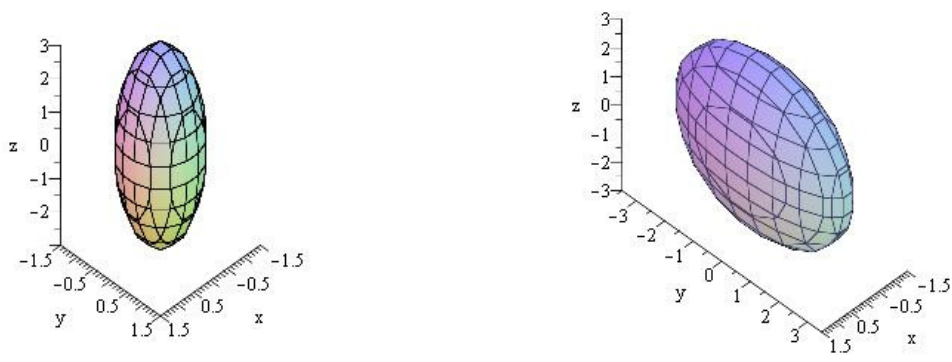

Figure 3.2: Prolate (left) and oblate (right) ellipsoids with centers at $\mathbf{r}=0$. Prolate semi-axes $a=1, b=1, c=3$ and oblate $a=1, b=3, c=3$.

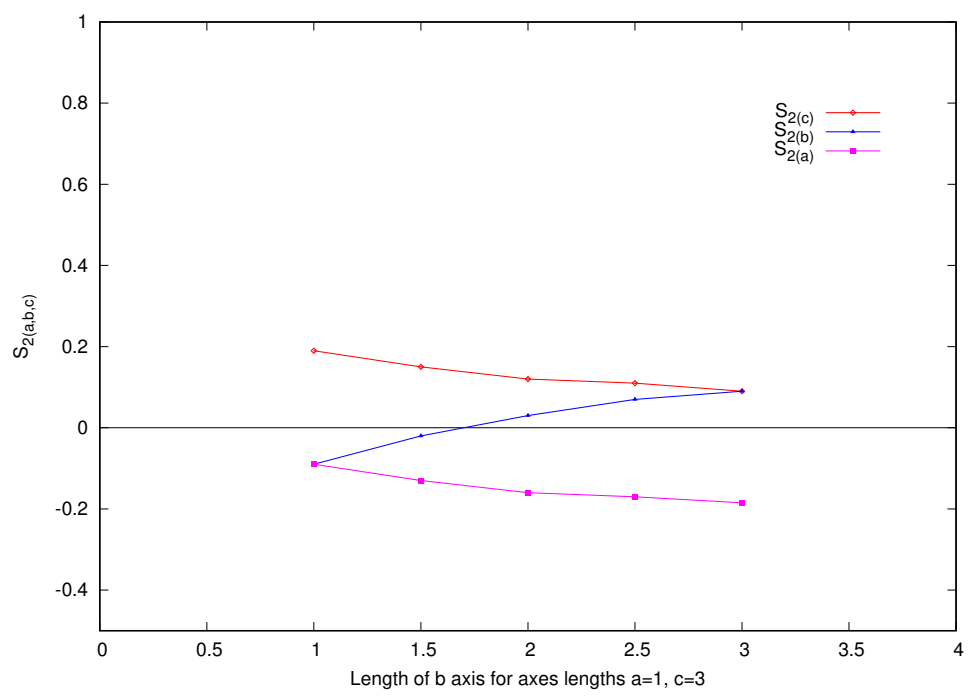

Figure 3.3: Order parameters $S_{2(a)}, S_{2(b)}, S_{2(c)}$ for the uniaxial ordering of microtubule length in an ellipsoid with axes a, $b, c$ as functions of the only axis with variable length $b$. Parameters: $a=1, c=3$ and the remaining as in Table 1. 


\begin{tabular}{lll}
\hline Parameter & Value & Reference \\
\hline$v_{+}$ & $0.018 \mu \mathrm{m} \mathrm{s}^{-1}$ & {$[52]$} \\
$v_{-}$ & $0.040 \mu \mathrm{m} \mathrm{s}^{-1}$ & {$[52]$} \\
$r_{n}$ & $0.05 \mathrm{~s}^{-1}$ & {$[53]$} \\
$r_{+}$ & $0.0078 \mathrm{~s}^{-1}$ & {$[52]$} \\
$r_{-}$ & $0.0016 \mathrm{~s}^{-1}$ & {$[52]$} \\
$r_{u}$ & $0.01 \mathrm{~s}^{-1}$ & {$[49]$} \\
\hline
\end{tabular}

Table 3.1: Model parameters.

At this point we explain why we chose the value $\bar{l}=2.54$ of the mean length in our calculations. The reason is that this value is close to the longest distance $(c=3)$ to the boundary, which is a necessary requirement in order to take into consideration the interaction of microtubules with the most distant boundary regions.

\subsubsection{Axially symmetric cell with a central centrosome}

\subsubsection{Analytical approach}

Having in mind the comparison between theory and 2D-simulations, which are simpler and faster than the 3D ones, we examine here the sensitivity of the ordering degree when we change the model from 3D to 2D. To this end, we compare a $3 \mathrm{D}$ ellipsoid of revolution with axes half-lengths $a=b<c$, with the elongated direction along the $z$-axis, with a 2D ellipse with axes half-lengths $a<c$, with the long axis now along the $x$ direction. In both cases we call the polar angle with respect to the long axis $\theta$. We now note that

$$
L^{(3)}(\theta, \phi)=L^{(3)}(\theta)=\frac{a c}{\sqrt{a^{2} \cos ^{2} \theta+c^{2} \sin ^{2} \theta}}=L^{(2)}(\theta) \equiv L(\theta)
$$

This implies that as a function of $\theta$ the results for the length distributions in the $2 \mathrm{D}$ and the $3 \mathrm{D}$ cases are identical, i.e.

$$
l^{(3)}(\theta)=l^{(2)}(\theta)=\frac{\bar{t} \bar{l}\left(1-F_{b}(\theta)\right)+\left(t_{b}-\bar{t}\right) L(\theta) F_{b}(\theta)}{t_{0}+\bar{t}\left(1-F_{b}(\theta)\right)+t_{b} F_{b}(\theta)} \equiv l(\theta)
$$


We thus need to compare

$$
\begin{aligned}
S_{2}^{(2)} & =\frac{\int_{0}^{\pi} d \theta \cos (2 \theta) l(\theta)}{\int_{0}^{\pi} d \theta l(\theta)} \\
S_{2}^{(3)} & =\frac{\int_{0}^{\pi} d \theta \sin (\theta) P_{2}(\cos \theta) l(\theta)}{\int_{0}^{\pi} d \theta \sin (\theta) l(\theta)}
\end{aligned}
$$

where in the $2 \mathrm{D}$ case we have used that by symmetry $l(\theta+\pi)=l(\theta)$. This shows that in this setting the $2 \mathrm{D}$ case rigorously reproduces the $3 \mathrm{D}$ case, in the sense that the length-distribution is identical.

A numerical analysis is applied to a prolate ellipsoid and the ellipse that generates it by rotation for the set of parameters of Table 3.1. The comparison is made for the ordering degree in the direction of the long axis, which is common for the two shapes, and for different values of its length. The numerical results are shown in figure 3.4 and reveal that $S_{2}^{(2)} \geq S_{2}^{(3)}$ by a factor $\sim 2$ at intermediate ordering. The difference between the order parameters is simply due to the difference in their definition, since the analytical form of length distributions is the same.

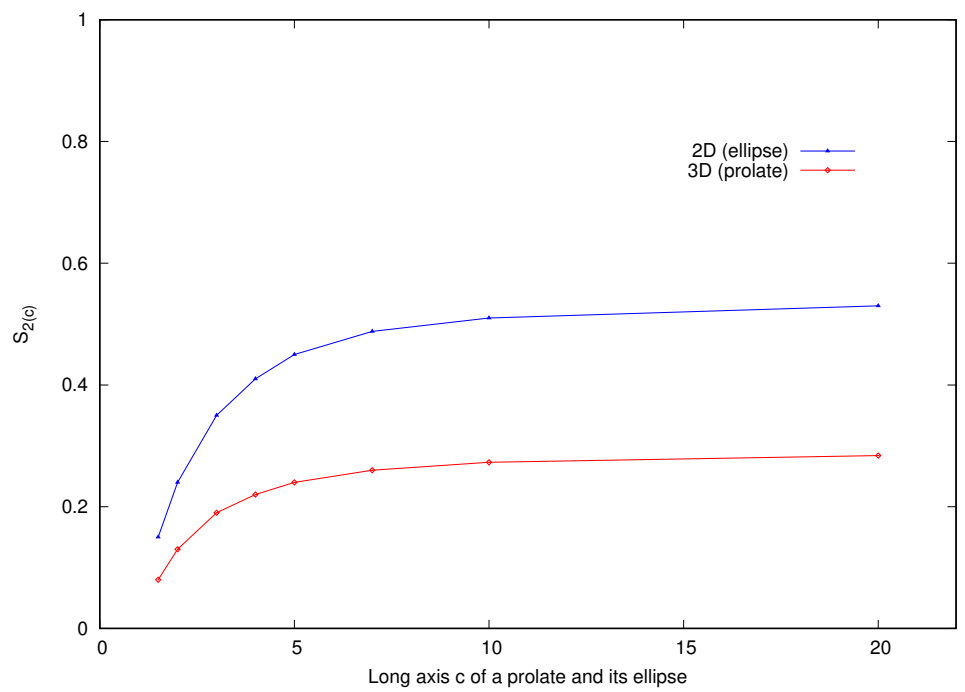

Figure 3.4: 3D and 2D order parameters for a prolate ellipsoid and the ellipse that generates it, as functions of the long axis length. Parameters: short axes equal to 1 and the remaining as in Table 3.1. 


\subsubsection{The effect of the residence time at the boundary and the mean length on $2 \mathrm{D}$ ordering.}

Here, we are going to calculate numerically the order parameter $S_{2}$ for the case of a finite unbinding rate $r_{u}$ and the case of no binding at the boundary of an ellipse with $a, b$ as the short and the long semi-axes (see figure 3.5). For each one of these cases we will regard two subcases, one with $a<\bar{l}$ and one with $a>\bar{l}$ for a certain value of $\bar{l}$. Then, for each sub-case we are going to regard four sub-sub-cases of different $b$ values.

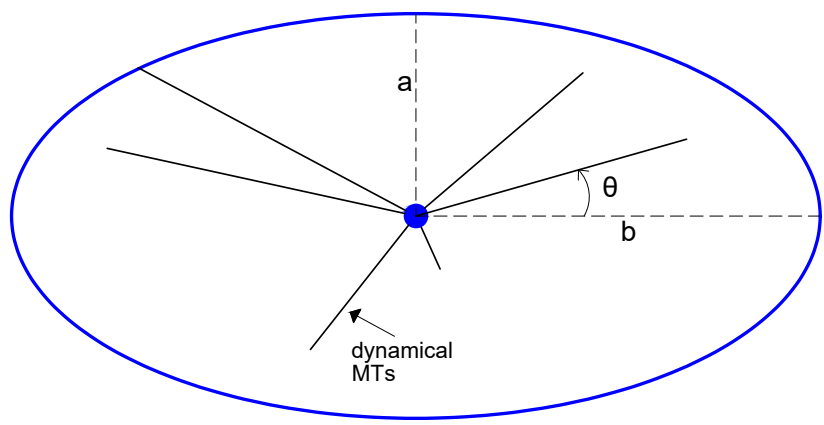

Figure 3.5: Schematic of the model applied to an ellipse. We can see the dynamical microtubules nucleated from a centrosome positioned at the center of the ellipse with semi-axes a, b. The nucleation angle $\theta$ of a single microtubule is shown, too.

We calculate now the order parameter $S_{2}$ for the case of no binding at the boundary and the case of a residence time $t_{b}$ at the boundary larger than $\bar{t}$. These cases are combined with the cases of a shorter or a longer than $\bar{l}$ short-axis a. 


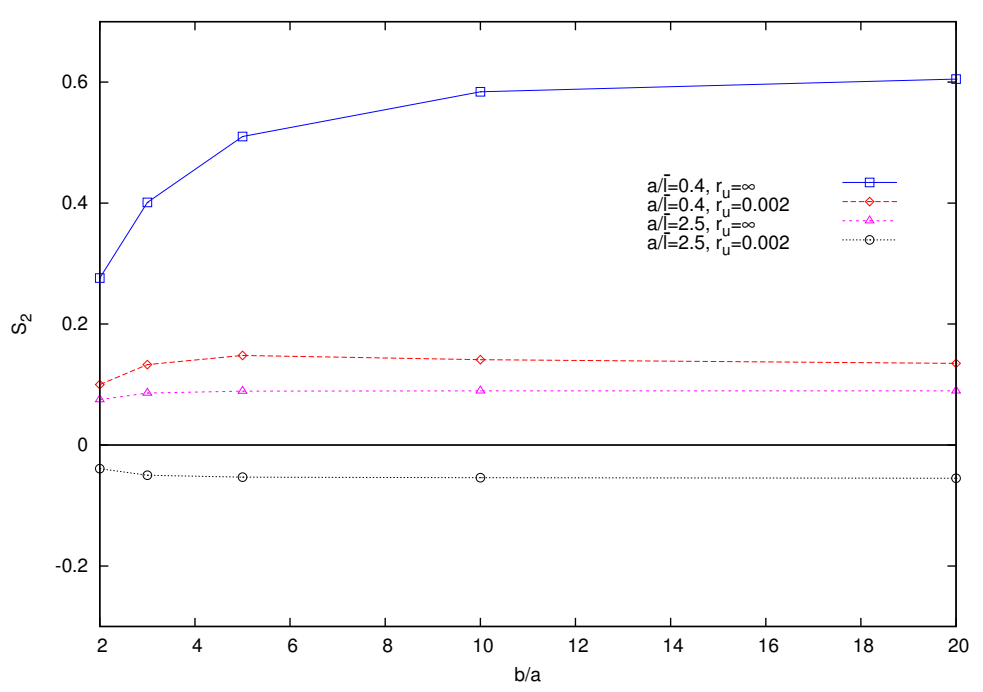

Figure 3.6: Order parameter $S_{2}=<\cos 2 \theta>$ as a function of $b / a$. The cases of a finite $\left(r_{u}=0.002 s^{-1}\right)$ and an infinite binding rate are examined in combination with the cases of a shorter $(\mathrm{a}=1 \mu \mathrm{m})$ and a longer $(\mathrm{a}=6.35 \mu \mathrm{m})$ short-axis than $\bar{l}$. Parameters: $r_{n}=0.05 \mathrm{~s}^{-1}, \bar{l}=2.54 \mu \mathrm{m}, \bar{t}=205 \mathrm{~s}$.

Looking at the results shown in figure (3.6), we can see that the long residence time of microtubules at the cell boundary lowers the degree of longitudinal ordering and, in combination with longer short-axes in relation to the mean length $\bar{l}$, changes ordering to transverse. So, low unbinding rates and higher ratios of short-axis to mean length work together to the direction of the transverse ordering. Furthermore, we can see that the axes ratio $b / a$ affects significantly ordering when the short axis is shorter than the mean length of microtubules and there is no binding to the boundary.

\subsubsection{Comparison with 2D-simulations}

In order to verify the theoretical results, we proceed to computer simulations. In the simulation algorithm we regard the states DORMANT, GROWING and SHRINKING for the microtubules that do not touch the cell boundary. We also consider the state BOUND for the microtubules that are stalled to the boundary.

The DORMANT state is the state after which a microtubule comes to 
the GROWING state. Regarding the GROWING state, it describes a growing microtubule that has not yet touched the boundary of the ellipse. So, the microtubule can continue to this state or it can go to a catastrophe and pass to the SHRINKING state or it can come to the boundary and pass to the BOUND state.

Regarding the SHRINKING state, there are also three possibilities for a microtubule. It can go to the DORMANT state by keep shrinking and coming to the centrosome site, it can go to the GROWING state by keep shrinking and undergo a rescue or it can simply stay in the same state. Regarding the BOUND state, a microtubule can unbind from the cell boundary going to the SHRINKING state or it can stay in the same state.

We regard an ellipse and $M$ angle bins equal to $2 \pi / M$. The angle position of each bin is $\theta_{m}=(m+1) 2 \pi / M, m=0,1 . . M-1$. For a certain set of parameters, we calculate the length density of microtubules at each bin and, following, we construct the graph of the length density as a function of the angle position. Furthermore, the order parameter $S_{2}$ for the bipolar degree of the length distribution of microtubules is calculated as

$$
S_{2}=\frac{\sum_{m=0}^{M-1} l_{m} \cos 2 \theta_{m}}{\sum_{m=0}^{M-1} l_{m}}
$$

where $l_{m}$ is the length density of microtubules at the m-th angle $\theta_{m}$.

On the other hand, the analytical length density of microtubules for the same set of parameters is calculated by Eq. (3.52). We apply this calculation for the same angular binning with the simulation model and we construct the graph of the theoretical length density. The two graphs, corresponding to theoretical and to simulation results, are shown in figure (3.7). By comparing them, we find a high level of qualitative agreement. The same holds for the order parameters values of simulation (Eq. (3.55)) and theory (Eq. (3.43) ), which are found to be equal to the value 0.449 .

In the chapters of Part III, where the cell polarity is examined, the number densities of bound microtubules play a significant role. So here, except of the length density study, we proceed also to the application of the number density equations for bound microtubules and their comparison with $2 \mathrm{D}$ simulations. First, we write equation $(3.32)$ for $2 \mathrm{D}$ bound space

$$
m_{b}(\theta)=m \frac{t_{b} F_{b}(\theta)}{t_{0}+\bar{t}\left(1-F_{b}(\theta)\right)+t_{b} F_{b}(\theta)}
$$




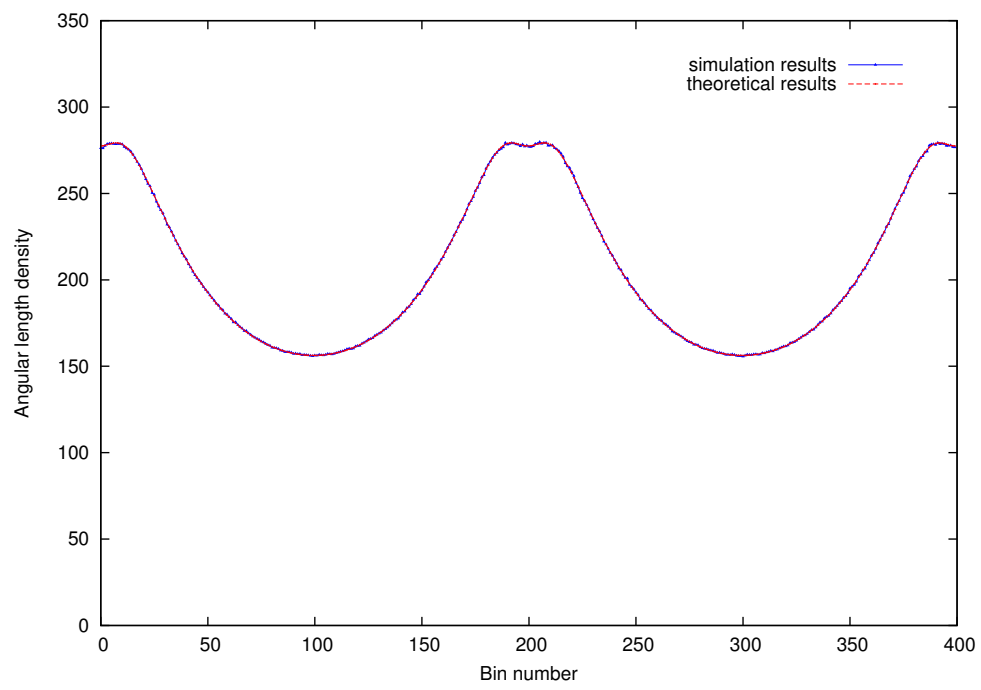

Figure 3.7: Verification of the theoretical length density by 2D simulations. The total angular length density ( $\mu \mathrm{m}$ per radian) of microtubules as a function of the angle bin number is shown. Parameters: long semi-axis $\mathrm{b}=4 \mu \mathrm{m}$, short semi-axis a $=2 \mu m, r_{n}, \bar{l}, \bar{t}$ as in figure (3.6), 1000 microtubules, 400 bins, $r_{u}=0.002 \mathrm{~s}^{-1}, 300 \cdot 10^{6}$ time steps equal to $0.5 \mathrm{~s}$ each.

Then, we apply this equation to an elliptical boundary for two chosen sets of parameters which differ only in the value of the unbinding rate $r_{u}$, getting the number density graphs of figure (3.8).

We can see that the number density of the bound microtubules is higher at sites of the boundary that are closer to the point of nucleation, as we expected taking into consideration the dynamics of microtubules. We can also see how the change of the unbinding rate at the boundary affects the distribution of the bound microtubules.

In order to verify the equation for the number density of bound microtubules nucleated from the center of an ellipse, we perform 2D simulations. Since the boundary is not circular, equal angles do not correspond to equal arc lengths. So, when representing boundary concentrations, we have to map the nucleation angles to arc lengths on the boundary. To that end, we choose to transform the nucleation angles $\theta$ of the polar coordinates to 


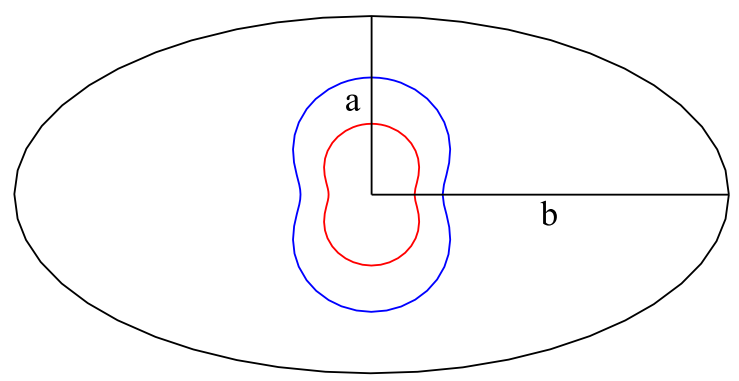

Figure 3.8: Number density of the bound microtubules nucleated from the center of an ellipse for two different unbinding rates $r_{u}=0.01 \mathrm{~s}^{-1}$ (blue color) and $r_{u}=0.02 s^{-1}$ (red color) at the boundary. The distance from the center equals fifteen times the value of the number density. Parameters: long semi-axis $b=3 \mu m$, short semi-axis $a=1.5 \mu m$ and the rest as in figure (3.7).

angles $\nu$ of the elliptic coordinates 54

$$
\nu=\arctan \left(\frac{\mathrm{b}}{\mathrm{a}} \tan \theta\right)
$$

Then, it's easy to find the arc length $s$ that corresponds to angle $\nu$

$$
s=\int_{0}^{\nu} d \nu^{\prime} \sqrt{\mathrm{a}^{2} \cos ^{2} \nu^{\prime}+\mathrm{b}^{2} \sin ^{2} \nu^{\prime}}
$$

Discretizing the ellipse boundary to a certain number of equal lengthed bins, we map each bin node length $s$ to a corresponding node angle, creating in that way a table of angles and corresponding arc lengths values. Then, a random angle of nucleation can be easily mapped by interpolation at the 
table values to an arc length that informs us about the bin number where it belongs.

We apply this procedure for a chosen set of parameters. The results are shown in figure (3.9), where the theoretical results are shown, too. The verification of theory by the simulations is obvious.

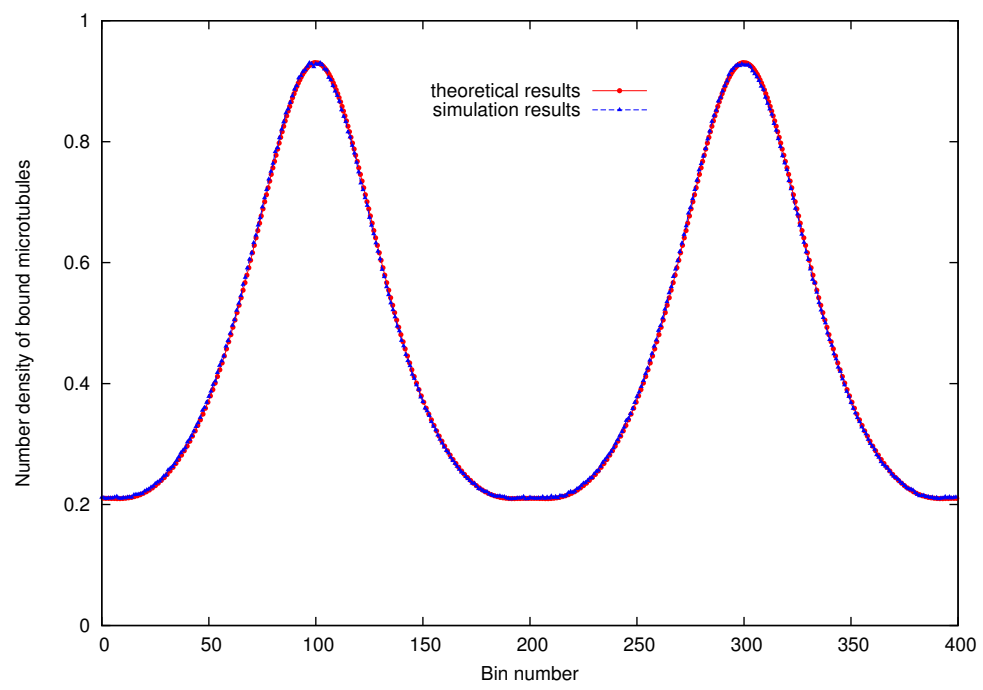

Figure 3.9: Verification of the theoretical number density of bound microtubules by $2 \mathrm{D}$ simulations. The number density of the bound microtubules as a function of the elliptical arc bin number is shown. Parameters: as in figure (3.7) for $10^{6}$ time steps equal to $0.5 \mathrm{~s}$ each.

\subsubsection{Non-central nucleation}

\subsubsection{Influence of the position of nucleation}

Here we examine how the position of the centrosome inside an elliptical cell affects the length distribution of microtubules. To that end, we regard different positions of the centrosome inside an ellipse. We apply Eq. (3.52) for the angular length density of the microtubules to five different nucleation sites inside an ellipse and we get five length distributions for the microtubules, which are shown in figure 3.10 . We can see the strong 
influence of the nucleation position to the length density distribution of the microtubules.

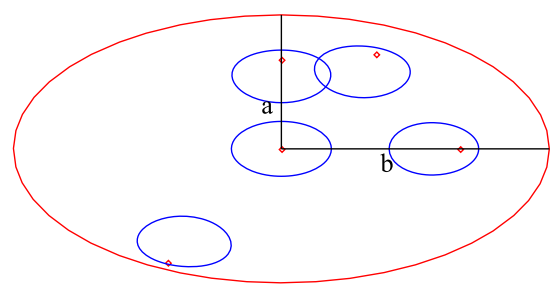

Figure 3.10: Total length density of microtubules nucleated from 5 different sites inside an ellipse. For each nucleation site the length density is $1 / 5$ of the distance from it. Parameters: as in figure (3.8), $r_{u}=0.01 s^{-1}$.

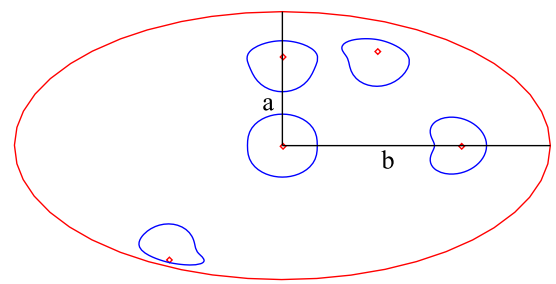

Figure 3.11: Length density of the bound microtubules nucleated inside an ellipse. For each nucleation site the length density is $1 / 10$ of the distance from it. Parameters: as in figure (3.8), $r_{u}=0.01 s^{-1}$.

Then, we calculate the length density distributions of the bound mi- 
crotubules that are nucleated from the same points inside the ellipse. To that end, we apply equation (3.36) for each nucleation site. The resulting distributions are shown in figure (3.11).

\subsubsection{Homogeneously distributed nucleation sites}

As we referred in the introduction, here we'll examine the case of noncentrosomal nucleation sites that are homogeneously distributed inside an ellipse. We determine the ordering behavior of the bound microtubules by performing stochastic simulations. To that end, we regard the order parameter

$$
S_{2}^{(\mathbf{r})}=\frac{\int d \mathbf{r} \int d \theta \cos 2 \theta l_{b}(\theta \mid \mathbf{r})}{\int d \mathbf{r} \int d \theta l_{b}(\theta \mid \mathbf{r})}
$$

where $\theta$ is the nucleation angle, $\mathbf{r}$ is the radial vector of the random nucleation site and $l_{b}(\theta \mid \mathbf{r})$ is the angular length density of the bound microtubules nucleated from site $\mathbf{r}$.

Due to complexity of calculating $S_{2}^{(\mathbf{r})}$ from the above equation, we use a numerical integration technique known as Monte Carlo integration. By regarding a finite number of nucleation points with random positions, the integration over the position vectors is replaced by the sum of the integrals of the nucleation points over the nucleation angle. So, the order parameter is read

$$
S_{2}^{(\mathbf{r})}=\frac{\sum_{i=1}^{n} \int d \theta \cos 2 \theta l_{b}\left(\theta \mid \mathbf{r}_{i}\right)}{\sum_{i=1}^{n} \int d \theta l_{b}\left(\theta \mid \mathbf{r}_{i}\right)}
$$

where $n$ is the total number of the nucleation sites.

The random positions of the nucleation sites inside the ellipse are considered to follow the homogeneous distribution. To that end, we must not select random values $r_{i}, \theta_{i}$ of the polar coordinates, as the elementary area is not equal to $d r d \theta$, but it's equal to $r d r d \theta$. So, we must use the cartesian coordinates $x_{i}, y_{i}$ for which the elementary area is $d x d y$.

We regard $\mathrm{a}, \mathrm{b}$ as the semi-axes of the ellipse with $\mathrm{a}>\mathrm{b}$. We also regard the random numbers $r_{x}, r_{y}$ with $r_{x}, r_{y} \in[-1,1]$, which generate the random points $\left(\mathrm{a} r_{x}, \mathrm{~b} r_{y}\right)$. These points must be at the interior of the ellipse, so the 
following condition must be satisfied

$$
\frac{\left(a r_{x}\right)^{2}}{a^{2}}+\frac{\left(b r_{y}\right)^{2}}{b^{2}}<1
$$

which becomes

$$
r_{x}^{2}+r_{y}^{2}<1
$$

We performed simulations for 6 different cases of axes ratio $b / a$ combined with 2 different values of short semi-axis a. The calculated values of the order parameter $S_{2}$ form the graphs of figure (3.12).

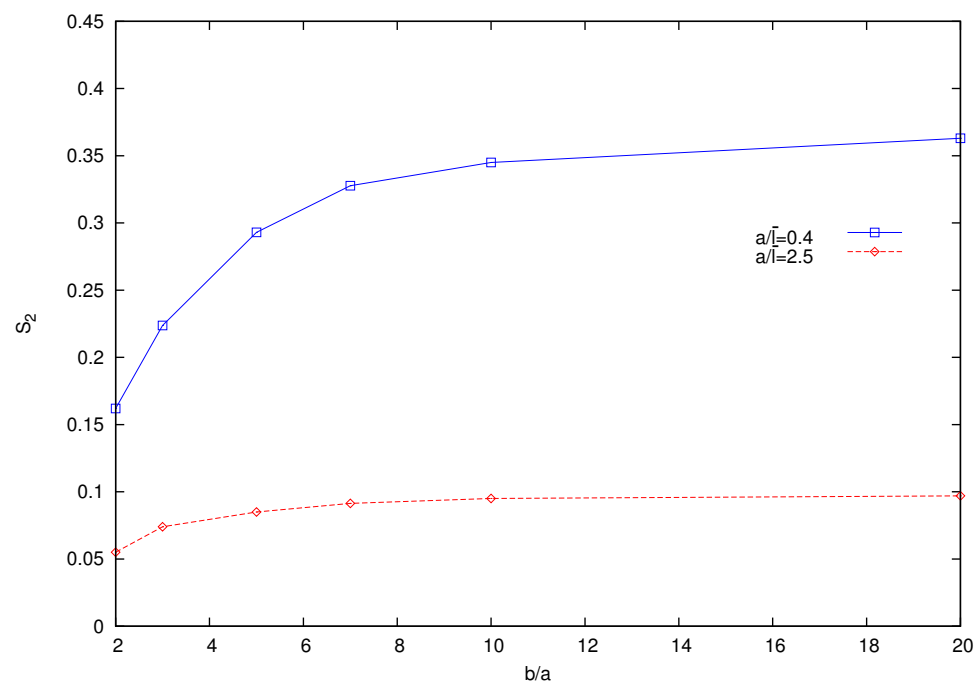

Figure 3.12: Order parameter $S_{2}$ as a function of $b / a$ for non-centrosomal nucleation sites homogeneously distributed inside an ellipse. The cases of a shorter $(\mathrm{a}=1 \mu \mathrm{m})$ and a longer $(\mathrm{a}=6.35 \mu \mathrm{m})$ short-axis than $\bar{l}$ are examined. Parameters: as in figure (3.7), $r_{u}=0.01 s^{-1}, 120$ nucleation points. 


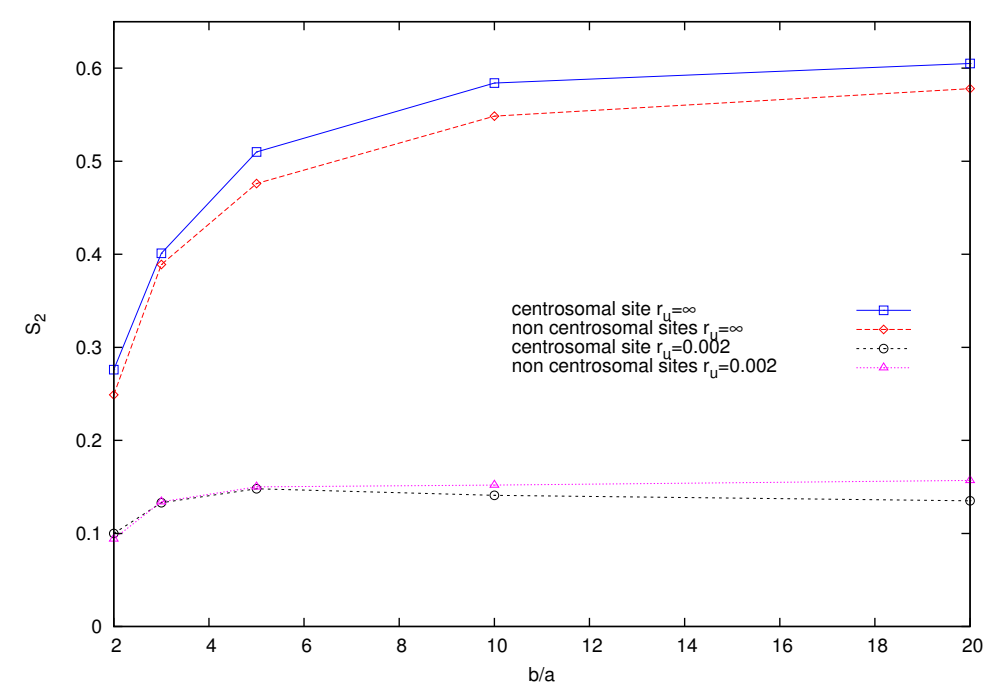

Figure 3.13: Order parameter $S_{2}$ as a function of $b / a$ for the case of a single centrosomal nucleation site at the center of an ellipse and the case of noncentrosomal nucleation sites homogeneously distributed inside an ellipse with $\mathrm{a}=1 \mu \mathrm{m}$, both examined for $r_{u}=\infty$ and $r_{u}=0.002 \mathrm{~s}^{-1}$. Parameters: as in figure (3.7), 120 nucleation points.

Looking at the graphs of figure (3.12), we conclude that as the short axis becomes longer than the mean length $\bar{l}$, the degree of ordering in the longitudinal direction becomes lower opening the way for ordering in the transverse direction. This is a corresponding conclusion with that of the case of a single centrosomal nucleation site.

A more detailed comparison of ordering between the case of a single centrosomal site at the center of an ellipse and the case of homogeneously distributed nucleation sites inside the ellipse is possible through the graphs of figure (3.13). For the case of an infinite unbinding rate and a certain set of parameters the $S_{2}$ parameter is calculated for different axes ratio. The shown results reveal the similar way that the shape affects ordering of centrosomal and non-centrosomal microtubules. 


\subsection{Conclusion}

In this chapter we have extended to $3 \mathrm{D}$ space a $1 \mathrm{D}$ model previously developed in [51] for microtubules which can stall at a boundary, and we derived the equations describing the length distribution of microtubules inside a cell. The equations were first formulated for a generic boundary shape. Subsequently, they were applied to the specific form of an ellipsoid of revolution with a centrosome at its center for which they could be solved numerically.

The presence of axial symmetry in the specific case of the ellipsoid made our model not very sensitive to a change of dimension, opening the way for a simpler and faster $2 \mathrm{D}$ simulation approach. We therefore regarded the subcase of a 2D ellipse with a centrosome at its center. An order parameter for the distribution of microtubules in 2D space was defined. By applying a numerical analysis of the equations, we revealed the domination of either a longitudinal or a transverse direction of ordering depending of the parameters of system. High unbinding rates and shorter short semiaxes in relation to the mean length of microtubules work to the direction of longitudinal ordering and the opposite combination leads towards the transverse ordering. A 2D simulation analysis was performed showing a high qualitative agreement between numerical and simulation results.

Going further with this model, we examined the influence of the nucleation position on the form of the length density distribution. To that end, we applied the equations extracted for a general form of a boundary to different nucleation sites inside an ellipse. In this way, we obtained different length density distributions, revealing a strong dependence on the position of nucleation.

We continued our analysis by regarding non-centrosomal nucleation points inside an ellipse. We defined an order parameter to calculate the degree of microtubule ordering and we approximated it by applying the method of Monte Carlo integration to a large number of spatially homogeneously distributed nucleation sites. By comparing the numerical results with the previously calculated ones for the single central nucleation site, we found that cell shape and the unbinding rate from the boundary have comparable effects on microtubule ordering. 


\title{
4 \\ A microtubule boundary-sliding model
}

\begin{abstract}
When a growing microtubule end hits the cell boundary, pushing forces acting on microtubules are generated. In non-spherical cells these forces lead to the slipping of microtubules along the plasma membrane. We develop a model to predict the effect of the sliding motion of microtubules on their orientational distribution. Unlike previously developed models for this effect, our model takes into account the dynamic nature of the pushing forces. Both an analytical treatment of a slightly perturbed spherical boundary and 2D stochastic simulations reveal a marked increase in the bipolar longitudinal ordering of the sliding microtubules.
\end{abstract}




\subsection{Introduction}

Here we develop a model for the phenomenon known as 'slipping' of the microtubules, which is described by the sliding of growing microtubule ends along non-spherical cell boundaries. Such 'slipping' of microtubules has been observed experimentally in vitro [55]. This motion of microtubules is the result of pushing forces acting on microtubules from the boundary, so to understand it we need to consider a force production mechanism. We call our model a 'boundary-sliding' model.

The interaction between microtubules and the cell boundary is crucial for the positioning of structures like the centrosome, the nucleus and the mitotic spindle. Pushing forces acting on microtubules when "hitting" the cell cortex are generated due to their polymerization [49, 56].

Furthermore, it is known that the growth velocity has quasi-statically an exponential decaying dependence on force [50]. We also know from a phenomenological model [57] that in steady state the mean time to catastrophe is a linear function of the growth velocity. Consequently, provided that different values of force are generated at the boundary, there is an obvious dependence of the catastrophe rate at the boundary on the values of the force acting on stalled microtubules.

One of the existing models, developed by Foethke et al. [58], takes into consideration the linear dependence of the catastrophe rate on the growth speed and the exponential decay of the growth speed with force. However, it doesn't predict theoretically the force values by assuming some force production mechanism, but uses force values observed in vitro. Another known model, due to Pavin and Laan [59], calculates the pushing forces on microtubules based only on the geometry of the confinement. This geometrical effect is expressed by the consistency between the growth speed of microtubules and their slipping speed on the boundary. Both these models neglect the dynamical nature of the pushing force acting on a microtubule that 'hits' the cell boundary.

Here we present a model, to date only briefly described in [60], which introduces the concept of a growing microtubule at the boundary "storing length", which causes it to be compressed following the Hooke's law. The resultant force has two effects, it slows down the further increase in length following the known force-velocity rule, and it increases the rate of catastrophe in keeping with the observed linear relation between life time 
and growth speed. Together these two effects self-consistently describe the dynamic character to the pushing force acting on the microtubules.

In the following, we'll look at how our model can predict the motion of the sliding microtubules and if any pattern of ordering is formed. To this end, we'll develop evolution equations for the sliding microtubules. Continuing, we are going to calculate analytically the distribution of the microtubules in the steady state at a boundary of certain 3D geometry. Next, we'll perform 2D stochastic simulations in order to investigate the existence of any ordering effect and, ending, we'll compare the results of our model with the work of Pavin et al. [59].

\subsection{The model}

\subsubsection{Force-production model}

We consider microtubules described by the standard two-state dynamical instability model. When not in contact with the boundary they switch between states in which they grow with speed $v_{+}$and shrink with speed $v_{-}$ - The rate of switching between growth and shrinkage in the unobstructed state is given by $r_{+}$(catastrophe) and the rate of switching between shrinking and growth by $r_{-}$(rescue).

We adopt the model developed in [60], which introduces the notion of the "stored length". According to this model, when a microtubule hits the fixed barrier at a distance $L$ from the nucleation site, it can still continue to grow as thermal fluctuations of the microtubule tip can open a gap for a new tubulin dimer to be added. The resultant compression of the elongating microtubule exerts a force on the barrier (an equal reaction force is of course exerted by the barrier on the microtubule) following Hooke's law

$$
F(l)=k(l-L)
$$

where $k$ is the compression modulus of the microtubule and $l-L$ is the stored length. 
a)

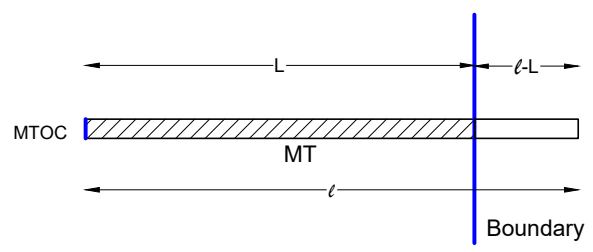

b)

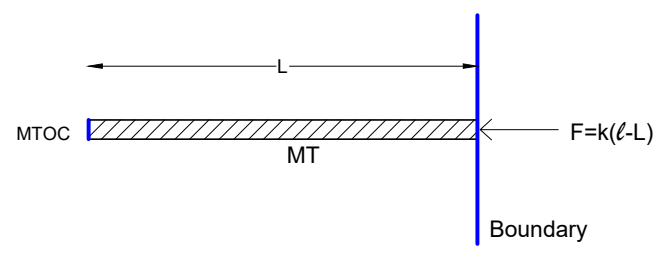

Figure 4.1: Schematic of the force-production model of "stored" length. a) Notion of a microtubule (MT) with length $l$ larger than the possible maximum length $L$ to the boundary. b) Equivalent system with a "stored" length $l-L$ on a microtubule with length $L$ and pushing force $F=k(l-L)$.

We next assume that the microscopic dynamics of the microtubule, involving the addition and removal of individual tubulin subunits, is fast compared to the growth process, and that the off-rate is small with respect to the on-rate. In that case the growth speed quasi-statically decreases as [50]

$$
\bar{v}_{+}(l)=v_{+} e^{-\beta d F}
$$

where $\beta=\left(k_{B} T\right)^{-1}$ is the inverse temperature in units of the Boltzmann constant and $d$ is the microscopic stepsize of the growth process. This parameter can be determined phenomenologically on the basis of measured force-velocity relations. The value suggested by [58] are

$$
\beta d=\frac{1}{f_{s}}=\frac{1}{1.67 p N}
$$

where $f_{s}$ is the sensitivity of the microtubule growth to the opposing force.

Throughout, we will use the overbar to denote quantities in the forceloaded state. The catastrophe rate should increase when in contact with the 
boundary. Lacking a detailed microscopic catastrophe model, we choose to extend the observation by Janson and Dogterom [57] that in steady state growth the mean time to catastrophe is proportional to the growth velocity, and we now assume that this also holds instantaneously. This implies that

$$
\bar{t}_{+}(l)=\frac{1}{\bar{r}_{+}(l)} \propto v_{+}(l)
$$

which yields

$$
\bar{r}_{+}(l)=r_{+} e^{\beta d F(l)}
$$

Moreover, we assume that in the loaded state, $l>L$, no rescues are possible, so that once a catastrophe occurs in this regime the microtubule will shrink to the unloaded state $l \leq L$ and that the shrinkage speed is unaffected by the loading.

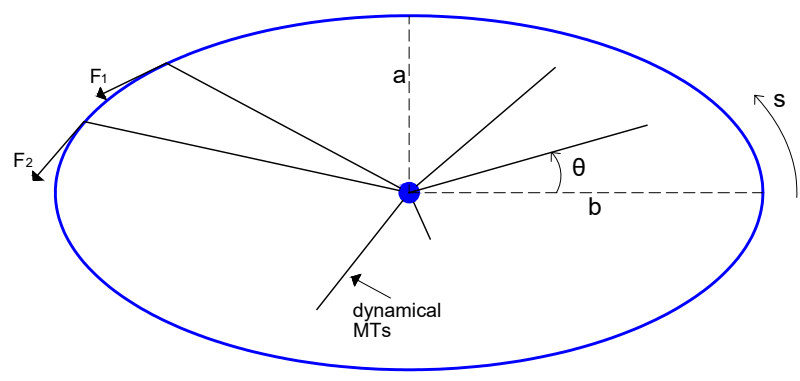

Figure 4.2: Schematic of the boundary-sliding model applied to an ellipse. The sliding force $F_{1}$ at the beginning and $F_{2}$ at the end of a 'sliding' step of a stalled microtubule is shown. We can see also the arc length coordinate $s$ and the nucleation angle $\theta$ of a single microtubule.

For non-spherical cells, the growth force is generically not perpendicular to the boundary and, so, has a tangential component $F_{\|}$. This component of the force is countered by the damping force of the microtubule-end's 
sliding motion on the boundary with velocity $v_{\|}$. Velocity and force are connected by the viscous equation of motion

$$
F_{\|}=-\xi v_{\|}
$$

where $\xi$ is the sliding drag coefficient.

\subsubsection{Evolution equations of sliding microtubules}

We proceed now to formulate the evolution equations which govern the sliding of the microtubules on the cell boundary. For simplicity reasons, we regard a convex cylindrically symmetric body, which will represent the cell shape in our analytical study. Due to the cylindrical symmetry, the points on the surface are parametrized by the radial distance $r(\theta)$, i.e. are only dependent on the polar angle $\theta$. The physical quantity for which we will develop evolution equations, is the number density $\bar{m}(l, \theta)$ of the sliding microtubules on the boundary.

Let $\delta A(\theta)$ be an (infinitesimal) element of surface area, $\hat{\mathbf{n}}(\theta)$ the unit vector perpendicular to it and $\hat{\mathbf{r}}(\theta)$ the unit vector parallel to the position vector $\mathbf{r}(\theta)$. We have

$$
\begin{aligned}
\mathbf{r}(\theta) & =r(\theta)(\sin \theta \cos \phi, \sin \theta \sin \phi, \cos \theta) \\
\hat{\mathbf{n}}(\theta) & =\frac{\frac{\partial \mathbf{r}}{\partial \theta} \wedge \frac{\partial \mathbf{r}}{\partial \phi}}{\left\|\frac{\partial \mathbf{r}}{\partial \theta} \wedge \frac{\partial \mathbf{r}}{\partial \phi}\right\|} \\
\hat{\mathbf{r}}(\theta) & =\frac{\mathbf{r}(\theta)}{r(\theta)}
\end{aligned}
$$

The projection of this element of area onto the sphere with radius $r=r(\theta)$ is $r^{2}(\theta) \sin \theta \delta \theta \delta \phi$, so

$$
\delta A(\theta)=\frac{r^{2}(\theta) \sin \theta \delta \theta \delta \phi}{\cos \psi(\theta)}
$$

with

$$
\cos \psi(\theta)=\hat{\mathbf{n}}(\theta) \cdot \hat{\mathbf{r}}(\theta)
$$




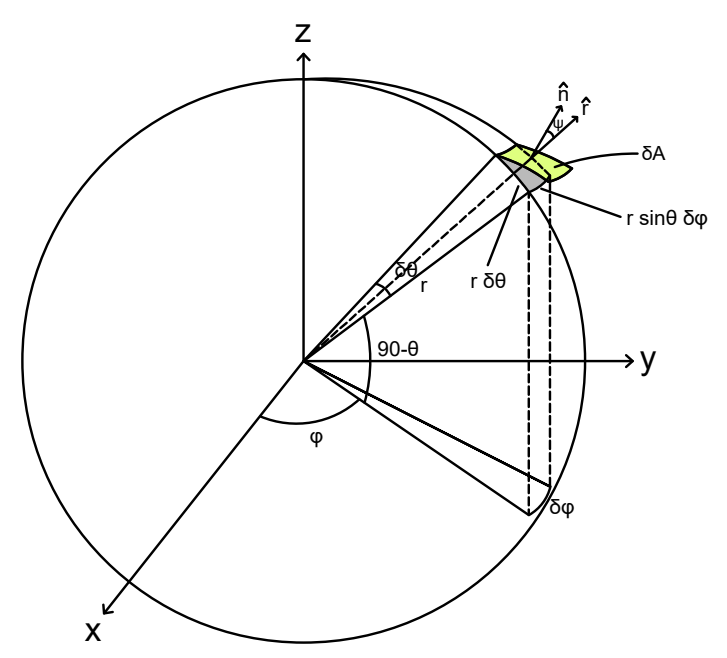

Figure 4.3: Elementary surface area $\delta A$ of a cylindrically symmetric body. The angle $\psi$ between the perpendicular to $\delta A$ unit vector $\hat{n}$ and the radial unit vector $\hat{r}$ is a function of the polar angle $\theta$ and correlates $\delta A$ to the spherical coordinates $r, \theta, \phi$.

For a small time step $\Delta t$, neglecting for the moment the catastrophe rate $r_{+}$on the boundary, we can consider that the number of sliding microtubules of the elementary surface area $\delta A$ is conserved. This conservation law can be expressed as

$$
\bar{m}(t+\Delta t, l+\Delta l, \theta+\Delta \theta) \delta A(\theta+\Delta \theta)=\bar{m}(t, l, \theta) \delta A(\theta)
$$

Substituting now $\delta A$ in 4.12 , we get

$$
\begin{array}{r}
\bar{m}(t+\Delta t, l+\Delta l, \theta+\Delta \theta) \delta l^{\prime} \delta \theta^{\prime} \frac{\sin (\theta+\Delta \theta) r^{2}(\theta+\Delta \theta)}{\cos \psi(\theta+\Delta \theta)}= \\
\bar{m}(t, l, \theta) \delta l \delta \theta \frac{\sin \theta r^{2}(\theta)}{\cos \psi(\theta)}
\end{array}
$$

We introduce the angular velocity $\omega(\theta)$ by using finite differences

$$
\omega(\theta)=\frac{\Delta \theta}{\Delta t}
$$


Then, the expanded finite differences $\delta l^{\prime}$ and $\delta \theta^{\prime}$ are

$$
\begin{aligned}
\delta l^{\prime} & =\delta l+\frac{\partial \bar{v}_{+}}{\partial l} \delta l \Delta t \\
\delta \theta^{\prime} & =\delta \theta+\frac{\partial \omega}{\partial \theta} \delta \theta \Delta t
\end{aligned}
$$

By definition, the angular velocity is given by

$$
\omega(\theta)=\frac{v_{\|}}{r(\theta)}
$$

So, we get

$$
\omega(\theta)=\frac{F_{\|}}{\xi r(\theta)}=\frac{k(l-r(\theta)) \sin \psi(\theta)}{\xi r(\theta)}
$$

We now define $g(\theta)$ as

$$
g(\theta) \equiv \frac{\delta A(\theta)}{\delta \theta \delta \phi}=\frac{\sin \theta r^{2}(\theta)}{\cos \psi(\theta)}
$$

Then, expanding the first part of (4.13) and $g(\theta)$ (see Appendix 4.6.1 to this chapter) and considering that there is a catastrophe rate $\bar{r}_{+}(l)$ of the microtubules at the boundary, we get

$$
\begin{array}{r}
\frac{\partial \bar{m}}{\partial t}(t, l, \theta)+\frac{\partial}{\partial l}\left(\bar{v}_{+} \bar{m}(t, l, \theta)\right)+\frac{1}{g(\theta)} \frac{\partial}{\partial \theta}(g(\theta) \omega(\theta) \bar{m}(t, l, \theta))= \\
-\bar{r}_{+} \bar{m}(t, l, \theta)
\end{array}
$$

which gives

$$
\begin{array}{r}
\frac{\partial \bar{m}}{\partial t}(t, l, \theta)=-\frac{\partial}{\partial l}\left(\bar{v}_{+} \bar{m}(t, l, \theta)\right)-\frac{1}{g(\theta)} \frac{\partial}{\partial \theta}(g(\theta) \omega(\theta) \bar{m}(t, l, \theta)) \\
-\bar{r}_{+} \bar{m}(t, l, \theta)
\end{array}
$$

Finally, in the steady state we have

$$
\frac{\partial}{\partial l}\left(\bar{v}_{+} \bar{m}(l, \theta)\right)=-\frac{1}{g(\theta)} \frac{\partial}{\partial \theta}(g(\theta) \omega(\theta) \bar{m}(l, \theta))-\bar{r}_{+} \bar{m}(l, \theta)
$$




\subsection{Analytical results}

We regard an ellipsoid of revolution that is a small perturbation of a sphere of radius $a$ and has equal surface area $S_{e}$ with the sphere surface area $S_{s}=4 \pi a^{2}$. The surface position where the pushing force is acting, can be found by considering the radial distance for a single elliptical generator of the ellipsoid

$$
r(\theta)=\frac{\bar{a}}{\sqrt{1-\epsilon^{2} \cos ^{2} \theta}}
$$

where $\bar{a}<b$ and the eccentricity $\epsilon=\sqrt{1-\left(\frac{\bar{a}}{b}\right)^{2}}<1$.

For the surface area of ellipsoid it holds

$$
S_{e}=2 \pi \bar{a}^{2}\left(1+\frac{b}{\bar{a} e} \sin ^{-1} \epsilon\right)
$$

and approximating $\sin ^{-1}$, we get

$$
S_{e}=4 \pi \bar{a}^{2}\left(1+\frac{1}{3} \epsilon^{2}\right)
$$

The equality between the surface area of the sphere $S_{s}=4 \pi a^{2}$ and the surface area of the ellipsoid gives

$$
\bar{a}=\frac{1}{\sqrt{1+\frac{1}{3} \epsilon^{2}}} a
$$

Then, substituting into 4.23 and approximating, we get

$$
r(\theta)=a\left(1+f_{1}(\theta) \lambda\right)
$$

where we introduce $\lambda=\epsilon^{2}<<1$ and $f_{1}(\theta)=\frac{1}{4}\left(\cos 2 \theta+\frac{1}{3}\right)$. We calculate

$$
\begin{aligned}
e^{-\beta d F(l, \theta)}=e^{-\beta d k(l-r(\theta))} & =e^{-k \beta d\left(l-a\left(1+f_{1}(\theta) \lambda\right)\right)} \\
& =e^{-k \beta d(l-a)}\left(1+k \beta d a f_{1}(\theta) \lambda\right)
\end{aligned}
$$

Similarly, we get

$$
e^{\beta d F(l, \theta)}=e^{-k \beta d(l-a)}\left(1-k \beta d a f_{1}(\theta) \lambda\right)
$$


Then, we get for the growth speed at the boundary

$$
\begin{aligned}
\bar{v}_{+} & =v_{+} e^{-k \beta d(l-a)}\left(1+k \beta d a f_{1}(\theta) \lambda\right) \\
\frac{\partial \bar{v}_{+}}{\partial l} & =-v_{+} k \beta d e^{-k \beta d(l-a)}\left(1+k \beta d a f_{1}(\theta) \lambda\right)
\end{aligned}
$$

We approximate the following quantities to the first order in $\lambda$

$$
\begin{aligned}
\sin \psi & =-\frac{1}{2} \lambda \sin 2 \theta \\
\cos \psi & =1 \\
\tan \psi & =-\frac{1}{2} \lambda \sin 2 \theta \\
f & =-\frac{k(l-a)}{2 a \xi} \lambda \sin 2 \theta \\
\frac{1}{g} \frac{\partial}{\partial \theta}(g f) & =-\frac{k(l-a)}{2 a \xi}(3 \cos 2 \theta+1) \lambda=f_{2}(l, \theta) \lambda
\end{aligned}
$$

with

$$
f_{2}(l, \theta)=-\frac{k(l-a)}{2 a \xi}(3 \cos 2 \theta+1)
$$

We set

$$
p=k \beta d
$$

Then, equation 4.22 becomes

$$
\begin{aligned}
& \left(v_{+} e^{-p(l-a)}\left(1+p a f_{1}(\theta) \lambda\right)\right) \frac{\partial \bar{m}}{\partial l}+\left(\frac{k(l-a)}{2 a \xi} \lambda \sin 2 \theta\right) \frac{\partial \bar{m}}{\partial \theta}+ \\
& \left(f_{2}(l, \theta) \lambda+p v_{+} e^{-p(l-a)}\left(1+p a f_{1}(\theta) \lambda\right)-r_{+} e^{p(l-a)}\left(1-p a f_{1}(\theta) \lambda\right)\right) \bar{m}=0
\end{aligned}
$$

Now, we perturb the density $\bar{m}^{(0)}(l)$ of the sphere to approximate the density $\bar{m}(l, \theta)$ at the ellipsoid to first order in $\lambda$

$$
\bar{m}(l, \theta)=\bar{m}^{(0)}(l)+\lambda \bar{m}^{(1)}(l, \theta)
$$


Following the formalism developed in Appendix 4.6.2, we get

$$
\bar{m}^{(0)}(l)=\frac{r_{n}}{v_{+}} e^{-\frac{a}{l}} e^{\frac{r_{+}}{2 p v_{+}}\left(e^{2 p(l-a)}-1\right)-p(l-a)}
$$

So, from 4.70 results

$$
\bar{m}^{(1)}(l)=\bar{m}^{(0)}(l) A(3 \cos 2 \theta+1)
$$

with

$$
A=-\frac{a r_{+}}{12 v_{+}} e^{2 p(l-a)}+\frac{k(l-a)}{2 a \xi v_{+} p}\left(e^{p(l-a)}-1\right)-\frac{1}{12}\left(2+\frac{a}{\bar{l}}-a p\right)
$$

Looking at equation 4.42, we conclude that for an ellipsoid that is a small perturbation to a sphere, a bipolar distribution of the bound microtubules is established.

\subsection{Simulations}

In order to examine the sliding effect on a more general boundary shape that is not a small perturbation to a sphere, we implement $2 \mathrm{D}$ computer simulations. To that end, we regard an elliptical cell boundary with a fixed centrosome positioned at its center. Since we are interested in the distribution of the microtubules that are stalled at the boundary, we use an arc length coordinate $s$ instead of the angle $\theta$. We discretize the cell boundary into a finite number of equal-length bins and we follow the procedure described in Chapter 3 to get arc length coordinates from the nucleation angles of microtubules. The finite time-step for a sliding event is chosen equal to $\Delta t=0.5 s$.

The 'stored length' dynamics of microtubules and the dynamic instability properties determine the states of the microtubules nucleated from centrosome. The pulling forces generated by motor proteins at the boundary are not taken into consideration, as they can not contribute to stress relaxation due to the the fact that we have pinned the location of the centrosome.

We regard the states DORMANT, GROWING and SHRINKING for the microtubules that do not touch the cell boundary. We also consider the states 
PUSH GROWING and PUSH SHRINKING connected with the force production mechanism. The last two states replace the BOUND state that we considered in Chapter 3 for microtubules that are bound to the cell boundary.

Regarding the GROWING state, it describes a growing microtubule that has not yet touched the boundary of the ellipse. So, the microtubule can continue in this state, or it can undergo a catastrophe and pass to the SHRINKING state, or it can reach to the boundary and pass to the PUSH GROWING state.

The DORMANT and the SHRINKING states are the same as the ones introduced in the algorithm of the anisotropic distribution of microtubules without sliding. So, a microtubule in the SHRINKING state can go to the DORMANT state, to the GROWING state or it can stay in the same state. A DORMANT microtubule can be nucleated into the GROWING state.

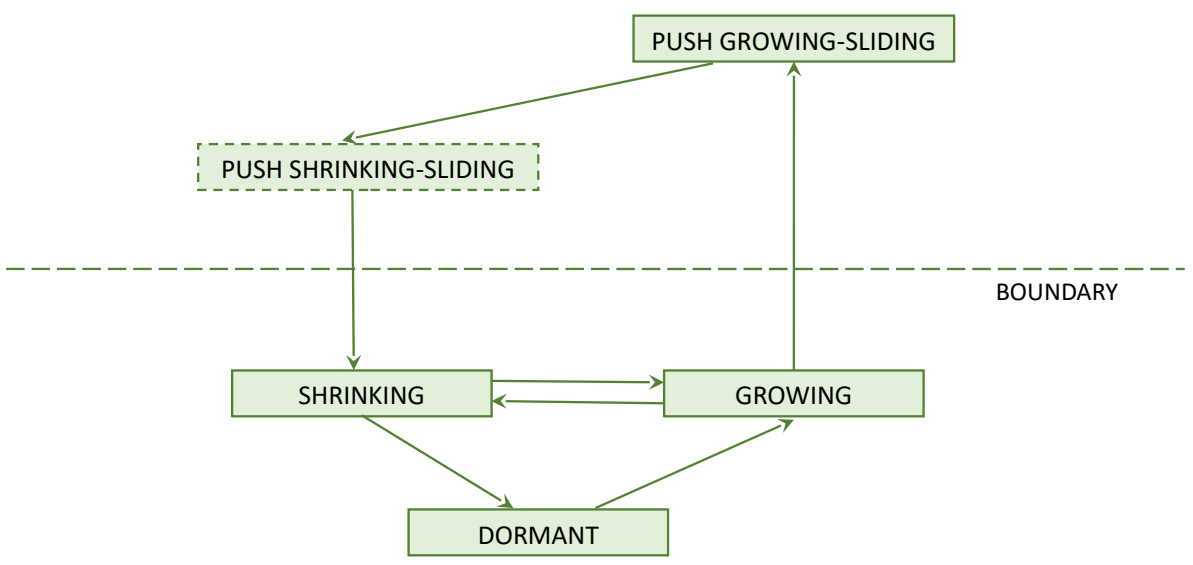

Figure 4.4: State diagram of the boundary-sliding model, which was applied to the simulations. The microtubule state "push shrinking-sliding" can be left out without any practical effect, as we show in the following.

The PUSH GROWING state describes a growing microtubule with a "stored 


\begin{tabular}{ll}
\hline Parameter & Simulation value \\
\hline $\mathrm{b}$ & $4 \mu \mathrm{m}$ \\
$\mathrm{a}$ & $2 \mu \mathrm{m}$ \\
$v_{+}$ & $0.018 \mu \mathrm{m} \mathrm{s}^{-1}$ \\
$v_{-}$ & $0.040 \mu \mathrm{m} \mathrm{s}^{-1}$ \\
$r_{n}$ & $0.05 \mathrm{~s}^{-1}$ \\
$r_{+}$ & $0.0078 \mathrm{~s}^{-1}$ \\
$r_{-}$ & $0.0016 \mathrm{~s}^{-1}$ \\
$\beta d$ & $0.60 \mathrm{pN}^{-1}$ \\
$k$ & $0.3{\mathrm{pN} \mu \mathrm{m}^{-1}}^{\mathrm{MTs}}$ \\
$\mathrm{M}$ & 1000 \\
\hline
\end{tabular}

Table 4.1: Model parameters. The elliptical boundary is divided to M equal bins, while the total number of microtubules is MTs.

length" $l>L$ and, so, a pushing force acting on it. The catastrophe rate of the microtubule at the boundary defines the future of a microtubule of this state. So, it can continue staying in this state or it can change to the PUSH SHRINKING state. If it stays in its state, then a sliding event takes place on the boundary defined by the value of the pushing force acting on the microtubule in the examined finite time step.

Finally, the PUSH SHRINKING state describes a shrinking microtubule with a "stored length" $l>L$ and, so, a pushing force still acting on it. We assume that no rescues occur at the boundary. A microtubule can therefore continue to stay in this state, causing a sliding event, or it can go to the SHRINKING state, if it leaves the boundary when its length is shorter than the radial distance to its current location on the boundary.

Our algorithm is applied to two sets of parameters, which differ only in the value of the sliding drag coefficient $\xi$. The remaining parameters are given in Table 4.1 .

As a result, we construct the two graphs of the distribution of boundary microtubules shown in figure 4.5, one for a high and one for a low sliding drag coefficient. Comparing the two graphs, we conclude that low sliding friction leads to a strong longitudinal ordering of the bound microtubules. 

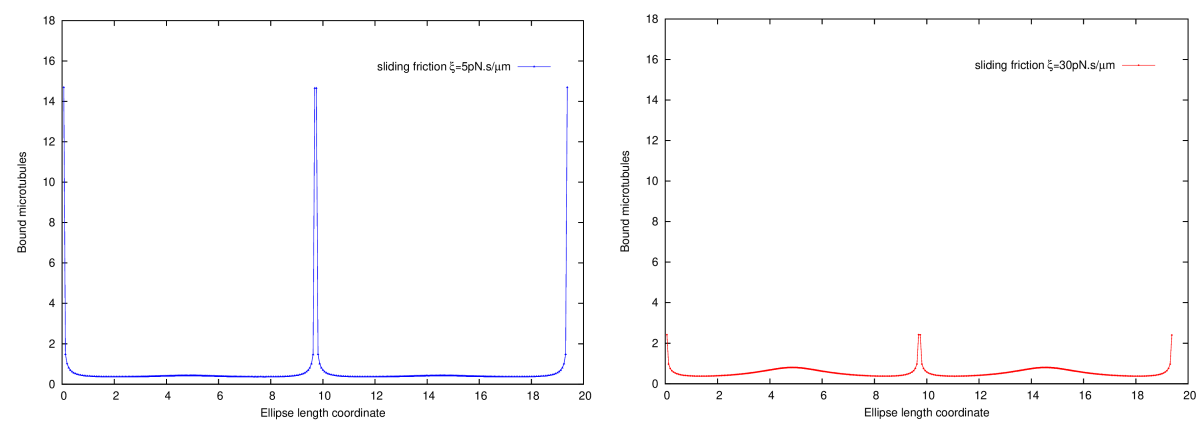

Figure 4.5: Distribution of microtubules on an elliptical boundary for a low $(\xi=5 \mathrm{pN} \mathrm{s} / \mu \mathrm{m}$, left) and a high $(\xi=30 \mathrm{pN} \mathrm{s} / \mu \mathrm{m}$, right $)$ sliding friction. The zero of the ellipse length coordinate is set at the edge of the long semi-axis. Remaining parameters: as in Table 4.1 .

We now ask whether the pushing force from the boundary after the microtubule switching to the shrinking state is in fact important. We therefore perform simulations in order to examine how the distribution of the bound microtubules is affected, if we set the pushing forces on bound microtubules that have just gone to the shrinking state to zero.

The simulation results in figure 4.6 show that there is a small difference between the case of force and no force acting on bound microtubules that are shrinking, which concerns mainly the number of bound microtubules at the longitudinal axis ends. We conclude that the influence of a nonzero pushing force applied after switching to shrinking state is practically negligible and, so, our previous results shown in figure 4.5 are essentially recovered.

At this point, we are going to compare the simulation results of our model with the corresponding ones of the model developed by Pavin and Laan [59]. To this end, and for a certain set of parameters, we regard an appropriate value for the compression modulus $k$ of our model in order to get the same mean pushing force per microtubule and the same mean number of microtubules for the two models. Then, the comparison between the two models is possible and leads to the results shown in figure 4.7 . 


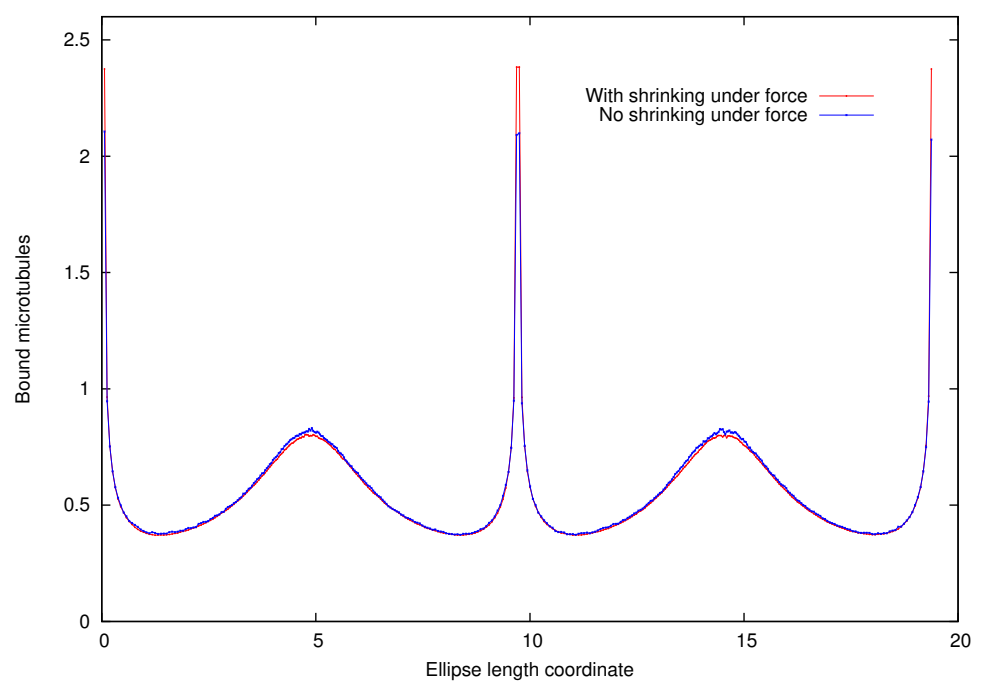

Figure 4.6: Distribution of microtubules on an elliptical boundary for the cases of a trivial (blue line) and a non-trivial (red line) pushing force acting on bound microtubules after switching to the shrinking state. The zero of the ellipse length coordinate is set at the edge of the long semi-axis. Parameters: sliding drag coefficient $\xi=30 \mathrm{pN} \mathrm{s} / \mu \mathrm{m}$ and the rest of the parameters as in figure 4.5 .

Looking at the microtubule distribution on the boundary for the two models that we examined, we notice that the overall degree of the longitudinal ordering is similar, however there are marked differences concerning the shape of the two distributions.

\subsection{Conclusion}

In this chapter we developed a model for the "sliding" of force-producing microtubule tips along the cell boundary. The force-production mechanism first proposed in [60] was considered as the driving force for this phenomenon. This mechanism takes into consideration the dynamic nature of force, which is influenced by the thermal fluctuations, the microscopic step size of the growth process and the stored length of a microtubule, which on the one hand generates an elastic force, but also increases the catastrophe 


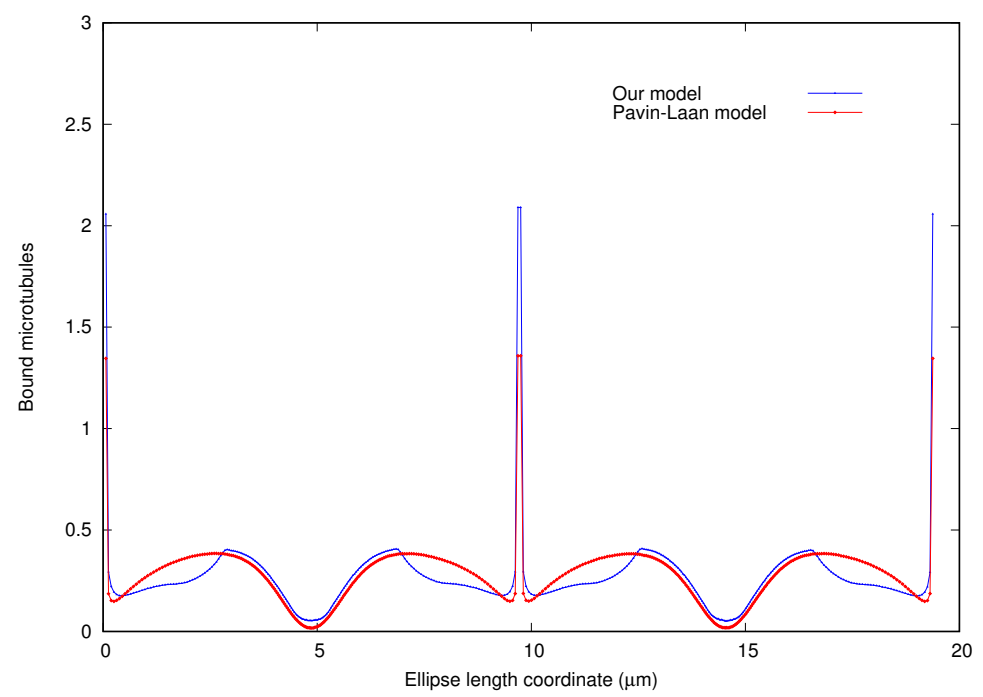

Figure 4.7: Distribution of microtubules on an elliptical boundary for our boundary-sliding model and the model of Pavin and Laan [59]. The two curves correspond to the same mean pushing force per microtubule $(0.7$ $\mathrm{pN}$ ) and the same mean number (104) of bound microtubules. The zero of the ellipse length coordinate is set at the edge of the long semi-axis. Parameters: sliding drag coefficient $\xi=5 \mathrm{pN} \mathrm{s} / \mu \mathrm{m}$, compression modulus $k=54 \mathrm{pN} / \mu \mathrm{m}$ and the rest of the parameters as in figure 4.5 .

rate. The resultant sliding motion of the microtubules is counteracted by the viscous exerted by the intracellular fluid..

The analysis assumed a cylindrical symmetry for simplicity reasons and lead to evolution equations for the number densities of sliding microtubules on the cell boundary. Due to the high complexity of the equations, even in the steady state, a full solution was not possible. Therefore only an ellipsoid of revolution very close to being spherical was considered, allowing a perturbative approach. This analysis revealed the establishment of a bipolar longitudinal distribution of the microtubules stalled to the boundary.

The longitudinal ordering of the sliding microtubules was verified in a more general elliptical shape by stochastic simulations. Both at the low and high friction a net longitudinal bipolar ordering was observed. However, in the high friction case the degree is markedly less, and the distribution over 
the surface shows clear signs of an underlying transverse ordering, as found in Chapter 3.

Finally, the boundary-sliding model developed here was compared with a competing model that considers pushing forces without a dynamic character [59]. The simulation analysis that was applied to the two models, revealed an overall similar degree of longitudinal ordering, suggesting that on a coarse scale the exact nature of the force-production mechanism is less important. However, the calculated distributions of boundary microtubules for the two models presented notable differences in the details of their shape, and hence to potentially testable predictions.

\subsection{Appendix}

\subsubsection{Expansions for the evolution equations}

Expanding now the first part of $(4.13)$, we get

$$
\begin{aligned}
& \left(\bar{m}(t, l, \theta)+\frac{\partial \bar{m}}{\partial l} \Delta l+\frac{\partial \bar{m}}{\partial \theta}(t, l, \theta) \Delta \theta+\frac{\partial \bar{m}}{\partial t}(t, l, \theta) \Delta t\right) \delta l^{\prime} \delta \theta^{\prime} g(\theta+\Delta \theta) \\
& =\left(\bar{m}(t, l, \theta) \delta l+\bar{m}(t, l, \theta) \frac{\partial \bar{v}_{+}}{\partial l} \delta l \Delta t+\frac{\partial \bar{m}}{\partial l}(t, l, \theta) \Delta l \delta l\right. \\
& \left.+\frac{\partial \bar{m}}{\partial \theta}(t, l, \theta) \Delta \theta \delta l+\frac{\partial \bar{m}}{\partial t}(t, l, \theta) \Delta t \delta l\right)\left(\delta \theta+\frac{\partial \omega}{\partial \theta} \delta \theta \Delta t\right) g(\theta+\Delta \theta) \\
& =(\bar{m}(t, l, \theta) \delta l \delta \theta+H(t, l, \theta) \delta \theta) g(\theta+\Delta \theta)
\end{aligned}
$$

with

$$
\begin{aligned}
& H(t, l, \theta)=\bar{m}(t, l, \theta) \delta l \frac{\partial \omega}{\partial \theta} \Delta t+\bar{m}(t, l, \theta) \frac{\partial \bar{v}_{+}}{\partial l} \delta l \Delta t+\frac{\partial \bar{m}}{\partial l}(t, l, \theta) \Delta l \delta l \\
& +\frac{\partial \bar{m}}{\partial \theta}(t, l, \theta) \Delta \theta \delta l+\frac{\partial \bar{m}}{\partial t}(t, l, \theta) \Delta t \delta l= \\
& \bar{m}(t, l, \theta) \delta l \frac{\partial \omega}{\partial \theta} \Delta t+\bar{m}(t, l, \theta) \frac{\partial \bar{v}_{+}}{\partial l} \delta l \Delta t+\frac{\partial \bar{m}}{\partial l}(t, l, \theta) \bar{v}_{+} \Delta t \delta l \\
& +\frac{\partial \bar{m}}{\partial \theta}(t, l, \theta) \omega \Delta t \delta l+\frac{\partial \bar{m}}{\partial t}(t, l, \theta) \Delta t \delta l= \\
& \frac{\partial}{\partial l}\left(\bar{v}_{+} \bar{m}(t, l, \theta)\right) \delta l \Delta t+\frac{\partial}{\partial \theta}(\omega(\theta) \bar{m}(t, l, \theta)) \delta l \Delta t+\frac{\partial \bar{m}}{\partial t}(t, l, \theta) \Delta t \delta l
\end{aligned}
$$


By expanding $g(\theta)$, 4.44 becomes

$$
\begin{aligned}
& (\bar{m}(t, l, \theta) \delta l \delta \theta+H(t, l, \theta) \delta \theta) g(\theta+\Delta \theta)= \\
& (\bar{m}(t, l, \theta) \delta l \delta \theta+H(t, l, \theta) \delta \theta)\left(g(\theta)+\frac{\partial g}{\partial \theta} \Delta \theta\right)= \\
& \bar{m}(t, l, \theta) \delta l \delta \theta g(\theta)+\bar{m}(t, l, \theta) \delta l \delta \theta \frac{\partial g}{\partial \theta} \Delta \theta+H(t, l, \theta) \delta \theta g(\theta)= \\
& g(\theta) \bar{m}(t, l, \theta) \delta l \delta \theta+\bar{m}(t, l, \theta) \delta l \delta \theta \frac{\partial g}{\partial \theta} \omega(\theta) \Delta t+g(\theta) \frac{\partial}{\partial l}\left(\bar{v}_{+} \bar{m}(t, l, \theta)\right) \delta l \Delta t \delta \theta \\
& +g(\theta) \frac{\partial}{\partial \theta}(\omega(\theta) \bar{m}(t, l, \theta)) \delta l \Delta t \delta \theta+g(\theta) \frac{\partial \bar{m}}{\partial t}(t, l, \theta) \Delta t \delta l \delta \theta= \\
& g(\theta) \bar{m}(t, l, \theta) \delta l \delta \theta+\frac{\partial}{\partial \theta}(g(\theta) \omega(\theta) \bar{m}(t, l, \theta)) \delta l \Delta t \delta \theta \\
& +g(\theta) \frac{\partial}{\partial l}\left(\bar{v}_{+} \bar{m}(t, l, \theta)\right) \delta l \Delta t \delta \theta+g(\theta) \frac{\partial \bar{m}}{\partial t}(t, l, \theta) \Delta t \delta l \delta \theta
\end{aligned}
$$

Then, 4.13) gives

$$
\begin{array}{r}
g(\theta) \frac{\partial \bar{m}}{\partial t}(t, l, \theta) \Delta t \delta l \delta \theta+g(\theta) \frac{\partial}{\partial l}\left(\bar{v}_{+} \bar{m}(t, l, \theta)\right) \delta l \Delta t \delta \theta \\
\frac{\partial}{\partial \theta}(g(\theta) \omega(\theta) \bar{m}(t, l, \theta)) \delta l \Delta t \delta \theta=0
\end{array}
$$

and, so,

$$
\begin{gathered}
\frac{\partial \bar{m}}{\partial t}(t, l, \theta) \Delta t \delta l \delta \theta+\frac{\partial}{\partial l}\left(\bar{v}_{+} \bar{m}(t, l, \theta)\right) \delta l \Delta t \delta \theta \\
+\frac{1}{g(\theta)} \frac{\partial}{\partial \theta}(g(\theta) \omega(\theta) \bar{m}(t, l, \theta)) \delta l \Delta t \delta \theta=0
\end{gathered}
$$

\subsubsection{Perturbation analysis}

Using equation 4.40, we calculate at the boundary $l=r(\theta)$ of the ellipsoid

$$
\begin{aligned}
\bar{m}(r(\theta), \theta) & =\bar{m}^{(0)}(r(\theta))+\lambda_{1} \bar{m}^{(1)}(r(\theta), \theta) \\
& =\bar{m}^{(0)}\left(a+f_{1}(\theta) \lambda a\right)+\lambda_{1} \bar{m}^{(1)}\left(a+f_{1}(\theta) \lambda a, \theta\right) \\
& =\bar{m}^{(0)}(a)+a \lambda f_{1}(\theta) \frac{\partial \bar{m}^{(0)}}{\partial l}(a)+\lambda_{1} \bar{m}^{(1)}(a, \theta)
\end{aligned}
$$

The number of microtubules per unit of surface area of the sphere should 
be equal to that of microtubules per unit of surface area of the ellipsoid:

$$
a^{2} \sin \theta d \theta d \phi \frac{r_{n}}{v_{+}} e^{-\frac{r(\theta)}{l}}=\bar{m}(l=r(\theta), \theta) \frac{\sin \theta r^{2}(\theta)}{\cos \psi(\theta)} d \theta d \phi
$$

which gives the general boundary condition

$$
\bar{m}(l=r(\theta), \theta)=\frac{a^{2} \cos \psi(\theta)}{r^{2}(\theta)} \frac{r_{n}}{v_{+}} e^{-\frac{r(\theta)}{l}}
$$

Using 4.27 and 4.51 we find

$$
\begin{aligned}
\bar{m}(l=r(\theta), \theta) & =\bar{m}\left(a\left(1+f_{1}(\theta) \lambda\right), \theta\right) \\
& =\frac{a^{2} \cos \psi(\theta)}{\left(a+f_{1}(\theta) \lambda a\right)^{2}} \frac{r_{n}}{v_{+}} e^{-\frac{a+f_{1}(\theta) \lambda a}{l}} \\
& =\frac{r_{n}}{v_{+}} e^{-\frac{a}{l}}+\lambda L^{(1)}(a, \theta)
\end{aligned}
$$

where

$$
L^{(1)}(a, \theta)=-\left(2+\frac{a}{\bar{l}}\right) f_{1}(\theta) \frac{r_{n}}{v_{+}} e^{-\frac{a}{l}}
$$

We combine equations 4.49 and 4.52 and get

$$
\begin{aligned}
\bar{m}^{(0)}(a) & =\frac{r_{n}}{v_{+}} e^{-\frac{a}{l}} \\
\bar{m}^{(1)}(a, \theta) & =L^{(1)}(a, \theta)-a f_{1}(\theta) \frac{\partial \bar{m}^{(0)}}{\partial l}(a)
\end{aligned}
$$

Equation 4.39 becomes after introducing 4.40

$$
\begin{aligned}
& \left(v_{+} e^{-p(l-a)}\left(1+p a f_{1}(\theta) \lambda\right)\right)\left(\frac{\partial \bar{m}^{(0)}}{\partial l}+\lambda_{1} \frac{\partial \bar{m}^{(1)}}{\partial l}\right)+ \\
& \left(f_{2}(l, \theta) \lambda+p v_{+} e^{-p(l-a)}\left(1+p a f_{1}(\theta) \lambda\right)-r_{+} e^{p(l-a)}\left(1-p a f_{1}(\theta) \lambda\right)\right) \\
& \left(\bar{m}^{(0)}+\lambda_{1} \bar{m}^{(1)}(l, \theta)\right)=0
\end{aligned}
$$


from which we get the following system of equations

$$
\begin{aligned}
& \frac{\partial \bar{m}^{(0)}}{\partial l}+\left(p-\frac{r_{+}}{v_{+}} e^{2 p(l-a)}\right) \bar{m}^{(0)}(l)=0 \\
& p a f_{1}(\theta) \frac{\partial \bar{m}^{(0)}}{\partial l}+f_{2}(l, \theta) \frac{1}{v_{+}} e^{p(l-a)} \bar{m}^{(0)}(l)+\frac{\partial \bar{m}^{(1)}}{\partial l}+ \\
& \left(p-\frac{r_{+}}{v_{+}} e^{2 p(l-a)}\right) \bar{m}^{(1)}(l, \theta)+p a f_{1}(\theta)\left(p+\frac{r_{+}}{v_{+}} e^{2 p(l-a)}\right) \bar{m}^{(0)}(l)=0
\end{aligned}
$$

which becomes

$$
\begin{aligned}
\frac{\partial \bar{m}^{(0)}}{\partial l}= & \left(\frac{r_{+}}{v_{+}} e^{2 p(l-a)}-p\right) \bar{m}^{(0)}(l) \\
\frac{\partial \bar{m}^{(1)}}{\partial l}= & \left(\frac{r_{+}}{v_{+}} e^{2 p(l-a)}-p\right) \bar{m}^{(1)}(l, \theta)-p a f_{1}(\theta) \frac{\partial \bar{m}^{(0)}}{\partial l} \\
& -\left(f_{2}(l, \theta) \frac{1}{v_{+}} e^{p(l-a)}+p a f_{1}(\theta)\left(p+\frac{r_{+}}{v_{+}} e^{2 p(l-a)}\right)\right) \bar{m}^{(0)}(l)
\end{aligned}
$$

Substituting 4.59) to 4.60), we get

$$
\begin{aligned}
\frac{\partial \bar{m}^{(1)}}{\partial l}= & \left(\frac{r_{+}}{v_{+}} e^{2 p(l-a)}-p\right) \bar{m}^{(1)}(l, \theta)-p a f_{1}(\theta)\left(\frac{r_{+}}{v_{+}} e^{2 p(l-a)}-p\right) \bar{m}^{(0)}(l) \\
& -\left(f_{2}(l, \theta) \frac{1}{v_{+}} e^{p(l-a)}+p a f_{1}(\theta)\left(p+\frac{r_{+}}{v_{+}} e^{2 p(l-a)}\right)\right) \bar{m}^{(0)}(l)
\end{aligned}
$$

We calculate

$$
\begin{aligned}
\frac{\partial \bar{m}^{(1)}}{\partial l}= & \left(\frac{r_{+}}{v_{+}} e^{2 p(l-a)}-p\right) \bar{m}^{(1)}(l, \theta) \\
& -\left(f_{2}(l, \theta) \frac{1}{v_{+}} e^{p(l-a)}+2 p a f_{1}(\theta)\left(\frac{r_{+}}{v_{+}} e^{2 p(l-a)}\right)\right) \bar{m}^{(0)}(l)
\end{aligned}
$$

We have

$$
\begin{aligned}
& f_{2}(l, \theta) \frac{1}{v_{+}} e^{p(l-a)}+2 p a f_{1}(\theta)\left(\frac{r_{+}}{v_{+}} e^{2 p(l-a)}\right)= \\
& -\frac{\xi k(l-a)}{2 a}(3 \cos 2 \theta+1) \frac{1}{v_{+}} e^{p(l-a)}+2 p a \frac{1}{4}\left(\cos 2 \theta+\frac{1}{3}\right)\left(\frac{r_{+}}{v_{+}} e^{2 p(l-a)}\right)= \\
& \left(\frac{p a r_{+}}{2 v_{+}} e^{2 p(l-a)}-\frac{3 \xi k(l-a)}{2 a v_{+}} e^{p(l-a)}\right) \cos 2 \theta+\left(\frac{p a r_{+}}{6 v_{+}} e^{2 p(l-a)}-\frac{\xi k(l-a)}{2 a v_{+}} e^{p(l-a)}\right)
\end{aligned}
$$


Now, equation 4.62 is written

$$
\begin{aligned}
\frac{\partial \bar{m}^{(1)}}{\partial l}+\left(p-\frac{r_{+}}{v_{+}} e^{2 p(l-a)}\right) \bar{m}^{(1)}(l, \theta)= & \left(-\frac{p a r_{+}}{6 v_{+}} e^{2 p(l-a)}+\frac{\xi k(l-a)}{2 a v_{+}} e^{p(l-a)}\right) \\
& (3 \cos 2 \theta+1) \bar{m}^{(0)}(l)
\end{aligned}
$$

We set

$$
Q(l, \theta)=\left(-\frac{p a r_{+}}{6 v_{+}} e^{2 p(l-a)}+\frac{\xi k(l-a)}{2 a v_{+}} e^{p(l-a)}\right)(3 \cos 2 \theta+1) \bar{m}^{(0)}(l)
$$

Then, 4.64 is written

$$
\frac{\partial \bar{m}^{(1)}}{\partial l}+\left(p-\frac{r_{+}}{v_{+}} e^{2 p(l-a)}\right) \bar{m}^{(1)}(l, \theta)=Q(l, \theta) \bar{m}^{(0)}(l)
$$

From 4.59) we get

$$
\bar{m}^{(0)}(l)=c_{1} e^{-\int_{a}^{l}\left(p-\frac{r_{+}}{v_{+}} e^{2 p\left(l^{\prime}-a\right)}\right) d l^{\prime}}
$$

and using 4.54

$$
c_{1}=\bar{m}^{(0)}(a)=\frac{r_{n}}{v_{+}} e^{-\frac{a}{l}}
$$

Then, we calculate from 4.66

$$
\begin{aligned}
\bar{m}^{(1)}(l)= & e^{-\int_{a}^{l}\left(p-\frac{r_{+}}{v_{+}} e^{2 p\left(l^{\prime}-a\right)}\right) d l^{\prime}} \\
& \left(\int_{a}^{l} e^{\int_{a}^{l}\left(p-\frac{r_{+}}{v_{+}} e^{2 p\left(l^{\prime}-a\right)}\right) d l^{\prime}} Q\left(l^{\prime}\right) c_{1} e^{-\int_{a}^{l}\left(p-\frac{r_{+}}{v_{+}} e^{2 p\left(l^{\prime}-a\right)}\right) d l^{\prime}} d l^{\prime}+c\right) \\
= & c_{1} e^{-\int_{a}^{l}\left(p-\frac{r_{+}}{v_{+}} e^{2 p\left(l^{\prime}-a\right)}\right) d l^{\prime}}\left(\int_{a}^{l} Q\left(l^{\prime}\right) d l^{\prime}+c_{2}\right)
\end{aligned}
$$

where $c_{2}=\frac{c}{c_{1}}$

This equation becomes with the help of (4.67)

$$
\bar{m}^{(1)}(l)=\bar{m}^{(0)}(l)\left(\int_{a}^{l} Q\left(l^{\prime}\right) d l^{\prime}+c_{2}\right)
$$

which gives

$$
\bar{m}^{(1)}(a)=\bar{m}^{(0)}(a) c_{2}=c_{1} c_{2}
$$


and using 4.55

$$
L^{(1)}(a, \theta)-a f_{1}(\theta) \frac{\partial \bar{m}^{(0)}}{\partial l}(a)=c_{1} c_{2}
$$

This equation gives with the help of 4.59) and 4.68

$$
L^{(1)}(a, \theta)-a f_{1}(\theta)\left(\frac{r_{+}}{v_{+}}-p\right) c_{1}=c_{1} c_{2}
$$

Using now 4.53 and 4.68, we get

$$
c_{2}(\theta)=-f_{1}(\theta)\left(a\left(\frac{r_{+}}{v_{+}}-p\right)+\left(2+\frac{a}{\bar{l}}\right)\right)
$$

Then, from 4.67 and 4.68 we calculate $\bar{m}^{(0)}(l)$ 


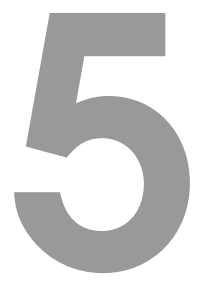

\section{Mitotic spindle positioning in cells}

The mitotic spindle plays a key role in cell division by segregating the duplicated chromosomes. Each of the two spindle poles is occupied by a centrosome, which nucleates microtubules. The interactions of centrosomal microtubules with the cell boundary are responsible for the positioning of the mitotic spindle in cell. In this chapter, we formulate a model that can predict the spindle positioning on the basis of these interactions. Our model takes into consideration the dynamic nature of the pushing forces acting on microtubules by the cell boundary, as well as pulling forces generated by cortical motor proteins. $2 \mathrm{D}$ stochastic simulations reveal the crucial role of the pulling forces to the stable positioning of the spindle. The transition between two stable orientation states is also examined for a model asymmetric cell shape. 


\subsection{Introduction}

The mitotic spindle is the cytoskeletal structure that segregates the duplicated chromosomes during eukaryotic cell division. At each of the two spindle poles a centrosome is positioned from which microtubules are nucleated, with different roles during mitosis. The structure of the spindle complex that is formed by the microtubules and the chromosomes is described next in our model.

It is known that the mitotic spindle interacts with the plasma membrane and both pushing [56] and pulling forces take place. The pushing forces are generated through microtubule polymerization. The predominant mechanism for the generation of pulling forces [26, 22, 23] is the one in which they are generated by dynein motors positioned at the cell cortex [22].

Here, we formulate a model that can predict the positioning of the mitotic spindle in the cell, based on the results of Chapter 3 and 4 of this thesis. It complements very recently published work [24] in which a model for the positioning and the orientation of the mitotic spindle was presented, which, however, did not consider the dynamic character of the pushing forces acting on microtubules.

We apply stochastic simulations, first to a 2D-elliptical confinement, in order to study the interplay of pushing and pulling pulling forces on the stability of spindle localization. Next, we consider spindle orientation in model asymmetric cell shape, a half-ellipse, constructing a phase diagram revealing the competition between different stable orientations.

\subsection{The model}

\subsubsection{Structure of the spindle complex}

The mitotic spindle is structured by microtubules with different roles. The kinetochore microtubules connect the two spindle poles with chromosomes at the kinetochores. The interpolar microtubules make antiparallel overlaps forming bundles that are bridging sister kinetochore microtubule fibers [61. The polar microtubules grow towards the spindle middle and their plus ends are free. The astral microtubules grow towards the plasma membrane and interact with it, ans so contribute to the positioning the spindle [62]. The 
spindle complex is illustrated in Figure 5.1 .

In our model we consider the part of the mitotic spindle without the astral microtubules as a rigid rod moving in a 2D-confinement. We are interested in the motion of the spindle as a whole, neglecting the interactions between kinetochore and interpolar microtubules or between antiparallel overlapping interpolar microtubules. The spindle motion is then determined by the interactions of the astral microtubules with the cell boundary. Furthermore, we consider the spindle length and width to be constant, thus we neglect any elongation effects. We chose a spindle-length to -width ratio equal to 3 , which is a realistic value [63].

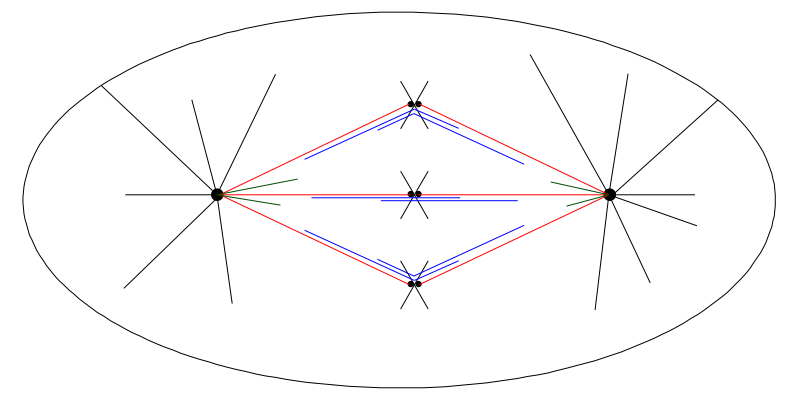

Figure 5.1: Schematic of the mitotic spindle complex [62. We can see the two centrosomes (big black circles) at the spindle poles, the astral microtubules (black line segments) nucleated towards the elliptical cell boundary, the kinetochore microtubules (red color) connecting the spindle poles with the kinetochores (small black circles) of the chromosomes, the interpolar microtubules (blue color) bridging sister kinetochore microtubules and the polar microtubules (green color).

Finally, we argue that due to stereochemical obstruction between each MTOC and the finite-width spindle axis (formed by both interpolar and kinetochore microtubules), the nucleation of microtubules from the two MTOCs at the spindle poles cannot be over the full 360 degrees range. We therefore assume that nucleation can not happen from a pole in directions which have an angle less than a minimal value value $\phi$ with the spindle axis. 


\subsubsection{Equations of motion}

In the cytoplasmic fluid viscous forces dominate over the inertial ones and for small enough velocities there exist a linear dependence between forces and velocities mediated by linear drag coefficients [64]. This results in the following equations of motion for our model spindle

$$
\begin{aligned}
F_{\perp} & =-\gamma_{\perp} v_{\perp} \\
F_{\|} & =-\gamma_{\|} v_{\|} \\
T & =-\gamma_{r} \omega
\end{aligned}
$$

where $F_{\perp}, F_{\|}$are the viscous drag forces perpendicular and parallel to the spindle axis, $v_{\perp}, v_{\|}$are the corresponding translational velocities, $T, \omega$ are the viscous torque and the rotational velocity of the spindle, $\gamma_{\perp}, \gamma_{\|}$stand for the translational drag coefficient in the perpendicular and the parallel direction to the spindle axis and $\gamma_{r}$ stands for the rotational drag coefficient. These viscous forces and torque counter the forces and corresponding torque that the astral microtubules apply on the spindle.

We express the drag coefficients $\gamma_{\perp}, \gamma_{r}$ in terms of $\gamma_{\|}$. Given the assumed geometry of our model spindle, we find the following relations (see the Appendix 5.5 for details of this derivation).

$$
\begin{aligned}
& \gamma_{\perp}=2 \gamma_{\|} \\
& \gamma_{r}=20 \gamma_{\|}
\end{aligned}
$$

What remains is to estimate the effective viscosity the spindle experiences. In the metaphase spindle, the non-kinetochore microtubules are crosslinked by proteins [65. The dynamics of the crosslinking leads to a structural rearrangement of the spindle. Hence the spindle exhibits an intrinsic effective viscosity, which is about 160 times larger than the cytoplasmic viscosity [66]. In our model, we consider that the motion of the mitotic spindle is highly affected by this effective viscosity, as the spindle is not a rigid body, but a network of components in a cytoplasmic pool. So, we regard a value of viscosity which is intermediate for the interval between the cytoplasmic viscosity and the internal viscosity of the spindle. We therefore choose a viscosity 70 times greater than the cytoplasmic one $(1.2 \mathrm{~Pa} \cdot \mathrm{s})$, equivalent to $\eta_{0}=84 \mathrm{~Pa} \cdot \mathrm{s}$. 


\subsection{Simulations}

\subsubsection{Simulation model}

In our model we adopt the force production mechanism introduced in the Chapter 4, which is based on the concept of the 'stored length'. Here, we combine this mechanism with the presence of pulling forces due to the effect of dynein motors present at the cell boundary. For simplicity reasons, however, we do not take into consideration the sliding of microtubules on the cell boundary. Unless otherwise indicated, we fix the minimum nucleation angle to be $\phi=45 \circ$.

Leaving out the sliding effect from the present simulation algorithm, we thus regard two boundary states of the microtubules. The first, PUSH GROWING in which pushing forces act on a bound microtubule, without sliding taking place. The second, which we call PULLING state, in which a constant pulling force of magnitude $F_{\text {pull }}$ is exerted on the microtubule.

A microtubule can go to the PULLING state from the PUSH GROWING state, if a dynein motor binds to it. Then, the microtubule stops growing and its length is taken to be equal to the distance between the centrosome and the boundary in accordance to recent experimental data [22]. We implement this by instantaneously setting the stored length to zero in the PULLING state of our model. The microtubule can stay in this state if the dynein motor stays bound to it or, otherwise, it can return to the SHRINKING state leaving the boundary. A complete state diagram including all the possible microtubule states and the routes between them is presented in figure 5.2

In the simulation model, by regarding finite time steps and displacements, the velocities of the Eqs. (5.1), (5.2) and (5.3) are given by

$$
\begin{aligned}
v_{\perp} & =\frac{\Delta y}{\Delta t} \\
v_{\|} & =\frac{\Delta x}{\Delta t} \\
\omega & =\frac{\Delta \phi}{\Delta t}
\end{aligned}
$$

where $\Delta x$ is the displacement parallel to the spindle axis, $\Delta y$ is the displacement vertically to the spindle axis and $\Delta \phi$ is the angle of rotation round the spindle middle. 


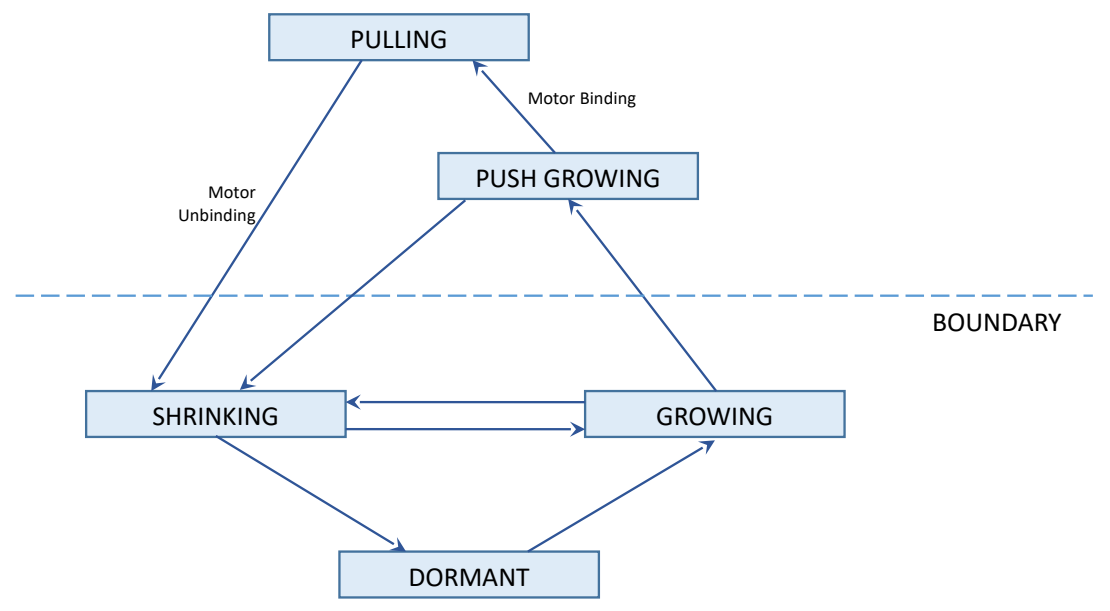

Figure 5.2: State diagram for the astral microtubules nucleated by the centrosomes at the two spindle poles.

Denoting by 1 and 2 the two spindle poles, the calculated absolute values of the net forces acting on these poles are $F_{1}, F_{2}$, the component forces parallel to the spindle axis $F_{1 x}, F_{2 x}$ and the component forces that are perpendicular to that axis $F_{1 y}, F_{2 y}$. Then, the net torque $T$ acting on the center of the spindle at each time step is calculated as

$$
T=\left(F_{1 y}-F_{2 y}\right) \frac{L_{s p i n}}{2}
$$

So, the rotation angle $\Delta \phi$ for this step is

$$
\Delta \phi=\frac{1}{\mu_{r}} T \Delta t
$$


The displacements of the spindle axis are calculated as

$$
\begin{aligned}
& \Delta x=\frac{1}{\mu_{\|}}\left(F_{1 x}+F_{2 x}\right) \Delta t \\
& \Delta y=\frac{1}{\mu_{\perp}}\left(F_{1 y}+F_{2 y}\right) \Delta t
\end{aligned}
$$

The last three equations determine the new position of the spindle for the next time step. We start most simulations with the spindle at an initial angle $\theta=45 \circ$ with respect to the long axis of the cell. The calculations are continued until the system relaxes to a steady state. There, the mean angle that is formed by the spindle and the long semi-axis of the half-ellipse shape becomes practically constant.

\subsubsection{Simulation geometries}

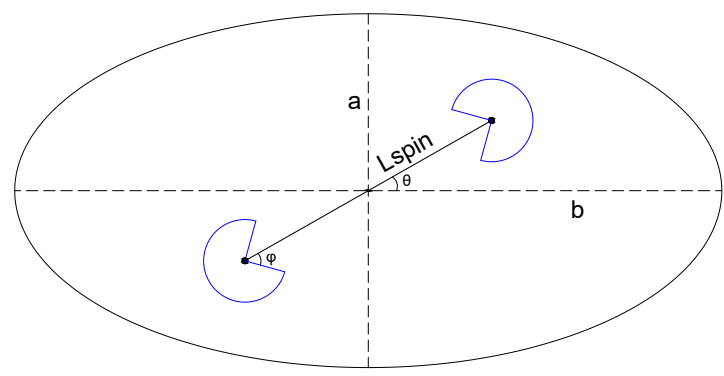

Figure 5.3: Microtubule nucleation angle distribution at the two spindle poles. We can see the elliptical cell boundary and the mitotic spindle with length $L_{\text {spin }}$. We can also see the short semi-axis a, the long semi-axis b, the orientation-angle $\theta$ of the spindle with $\mathrm{b}$ axis and the minimum nucleation angle $\phi$.

In order to investigate how the pulling forces affect the stability of positioning of the mitotic spindle, we first apply our model to an elliptical cell (figure 5.3 comparing the case of co-existing pushing and pulling forces 
with the case of pushing forces only. By suitably adjusting the basic parameter of the force-production model, the compression modulus $k$, we can achieve realistic values of the pushing forces. The impact of the pushing forces magnitude to the fluctuations of the spindle orientation is then also examined.

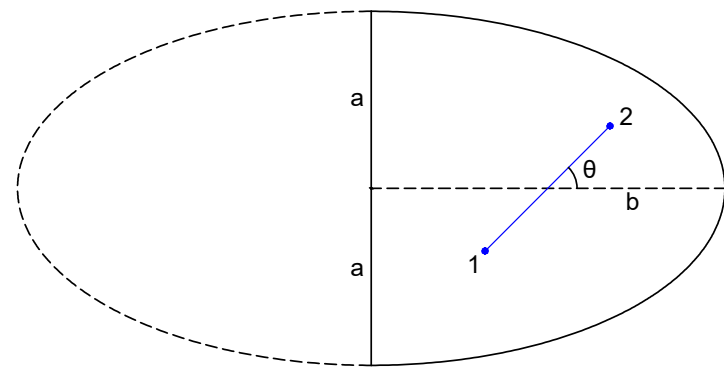

Figure 5.4: Cell boundary (solid line with black color) of a half-ellipse shape and mitotic spindle (blue color). We can see the short semi-axis length a, the long semi-axis length $\mathrm{b}$, the initial angle $\theta=\pi / 4$ of the spindle with $\mathrm{b}$ axis and the centrosomes 1 and 2 at the spindle poles.

Moreover, we further investigate how cell shape can influence the positioning of the mitotic spindle by applying our model to a cell geometry that does not possess double-axis symmetry. For that purpose we selected the half-ellipse shape. More specifically, the line segment defined by the two short semi-axes and the half elliptical perimeter connecting them, form the cell boundary inside which we regard the spindle. In the simulations we calculate the mean angle $\theta$ which spindle forms with the long semi-axis $\mathrm{b}$ in the steady state for different values of short semi-axis a. The cell geometry and the spindle inside it are shown in figure 5.4 .

\subsubsection{Results}

\subsubsection{Parametrizing the magnitude of the pushing forces}

Before we proceed with simulation analysis, we are going to show that our force-production mechanism can generate pushing forces of a realistic 
magnitude. To that end, we select a value for the compression modulus $(k=150 \mathrm{pN} / \mu \mathrm{m})$, which leads to an realistic [22] value of the mean maximum pushing force $(4.3 \mathrm{pN})$, in the presence of an also realistic value (5 $\mathrm{pN}$ ) of pulling force [22]. A histogram showing the distribution of the maximum pushing forces values corresponding to the previously referred mean value, is presented in figure 5.5 .

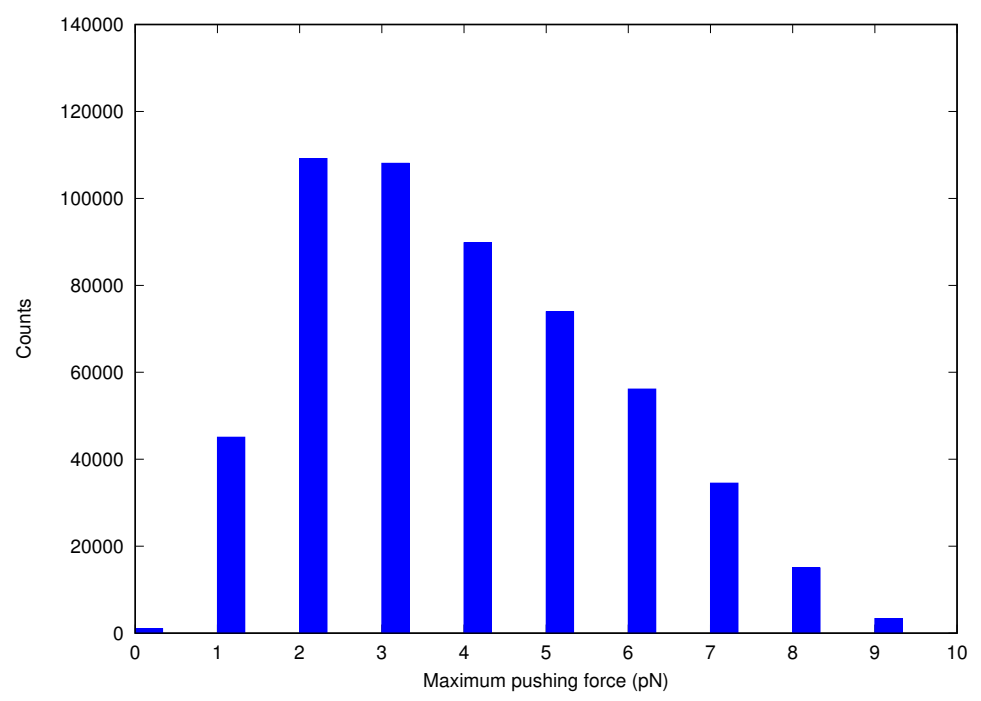

Figure 5.5: Distribution histogram of the maximum pushing force acting on microtubules for a compression modulus $k=150 \mathrm{pN} / \mu \mathrm{m}$. The calculated mean maximum pushing force equals to $4.3 \mathrm{pN}$, which is feasible 22]. Parameters: a $=4 \mu \mathrm{m}, \quad b=9 \mu \mathrm{m}, L_{\text {spin }}=6 \mu \mathrm{m}, \bar{L}=3.34 \mu \mathrm{m}, W=2 \mu \mathrm{m}$, $M=600, \quad d t=0.5 \mathrm{~s}, \quad \eta_{0}=84 \mathrm{~Pa} \cdot \mathrm{s}, \quad k_{b}=0.02 \mathrm{~s}^{-1}, \quad k_{o f f}=0.01 \mathrm{~s}^{-1}, \quad k_{n}=0.03 \mathrm{~s}^{-1}$, $\beta \delta=0.60 \mathrm{pN}^{-1}, F_{\text {pull }}=5 \mathrm{pN}, t=10^{6} \mathrm{~s}$.

\subsubsection{The role of pulling forces}

In order to investigate the role of the pulling forces in the stabilization of the mitotic spindle, we estimate the magnitude of the fluctuations of the spindle orientation angle in steady state. The standard deviation of this angle is calculated as a function of the compression modulus for the case of only pushing forces and the case of both pushing and pulling forces included in the system. The simulation results are shown in figure 5.6. The much 
higher fluctuations of a system with only pushing forces reveal the crucial role of the pulling forces in the stabilization of the mitotic spindle in cells.

Looking at the results of the figure 5.6, we can see that for a system that includes only pushing forces, the size of the fluctuations depends significantly on the value $k$ of the compression modulus. Specifically, as we increase $k$, the orientation fluctuations are becoming bigger. So, larger pushing forces work to the direction of destabilization.

In order to further analyze this effect, we calculate the distribution of the net torque acting on the spindle for two extreme values of the compression modulus $(k=5, k=150 \mathrm{pN} / \mu \mathrm{m})$. A histogram of the torque distribution is shown in figure 5.7. We can see that lower pushing forces (low $k$ ) are distributed mainly round low values of torque, while the distribution for higher pushing forces (high $k$ ) extends to larger torque values. This confirms the fact that large pushing forces increase the spindle orientation fluctuations.

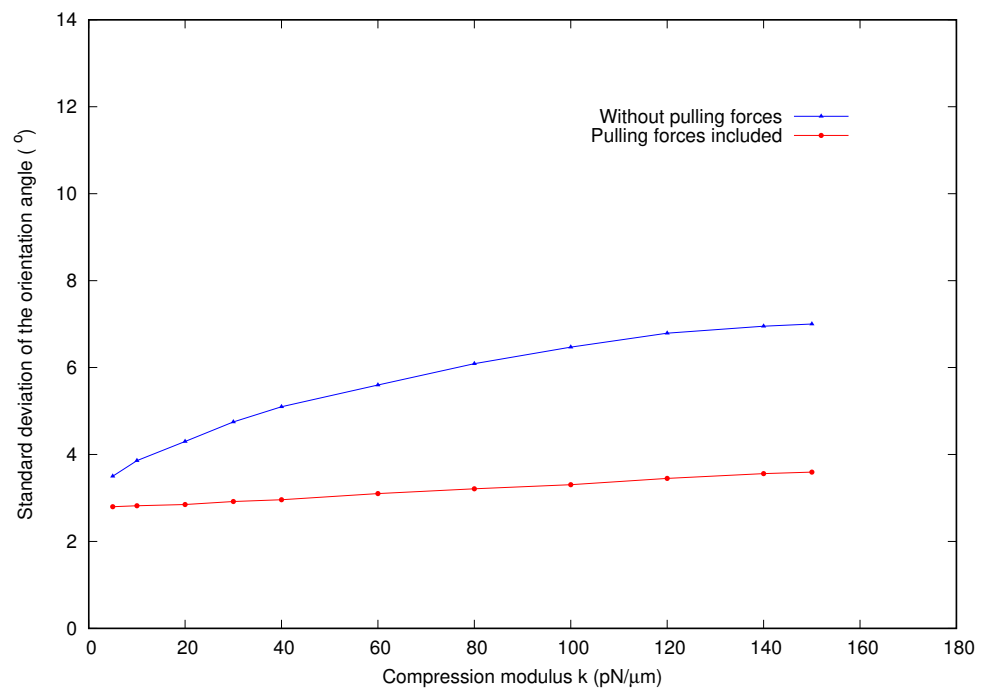

Figure 5.6: Standard deviation of the mean orientation angle as a function of the compression modulus for the cases with and without pulling forces. Parameters: as in figure 5.5 .

At this point, we are going to investigate why the pulling forces decrease the orientation fluctuations of the spindle. To this end, we calculate the distribution of the spindle torque with and without pulling forces for 


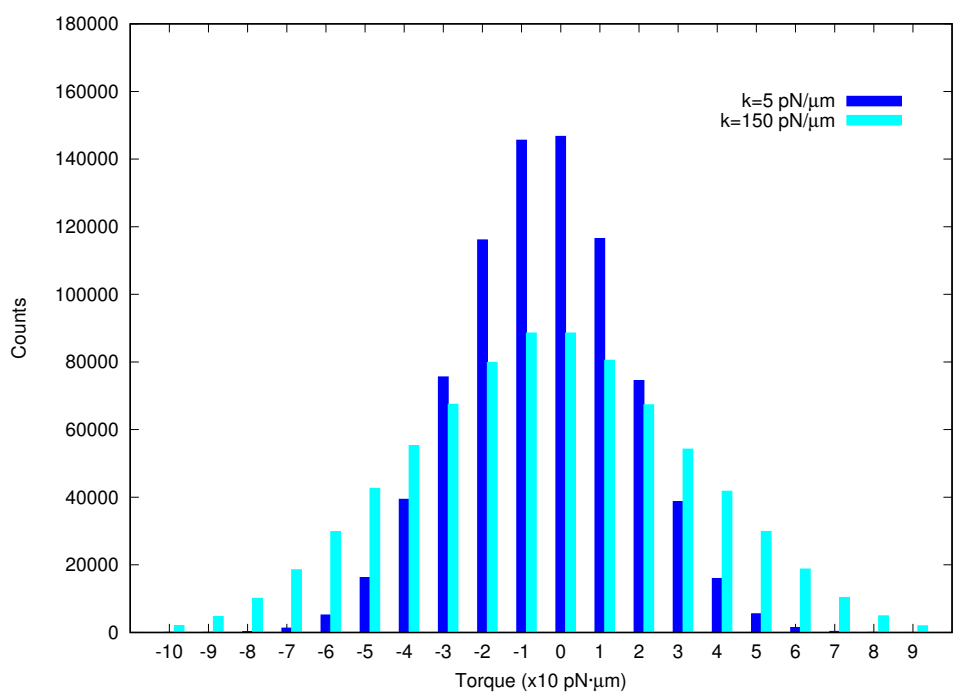

Figure 5.7: Distribution histogram of the torque acting on spindle for the cases of a low and a high value of compression modulus in the absence of pulling forces. Parameters: as in figure 5.5.

a certain value of the compression modulus. The simulation results are presented in the torque histogram of the figure 5.8, where the wider range of torque values when we have only pushing forces in the system verifies the stabilization of the spindle by the pulling forces.

The lower torque values explain indeed why the fluctuations are lower when the pulling forces are present. But how is this 'narrow' distribution of torque generated? First, we note that the pushing forces are reduced when we introduce pulling forces in the system. This is because pulling is an additional state option for the bound microtubules, so the residence time of the pushing microtubules at the boundary becomes less and, consequently, we get lower pushing force values. Second, when we regard pulling forces, we have more bound microtubules than if we have only pushing forces. This is a simulation result, which we present in figure 5.9. The higher number of bound microtubules increases the degree of the spindle stabilization. 


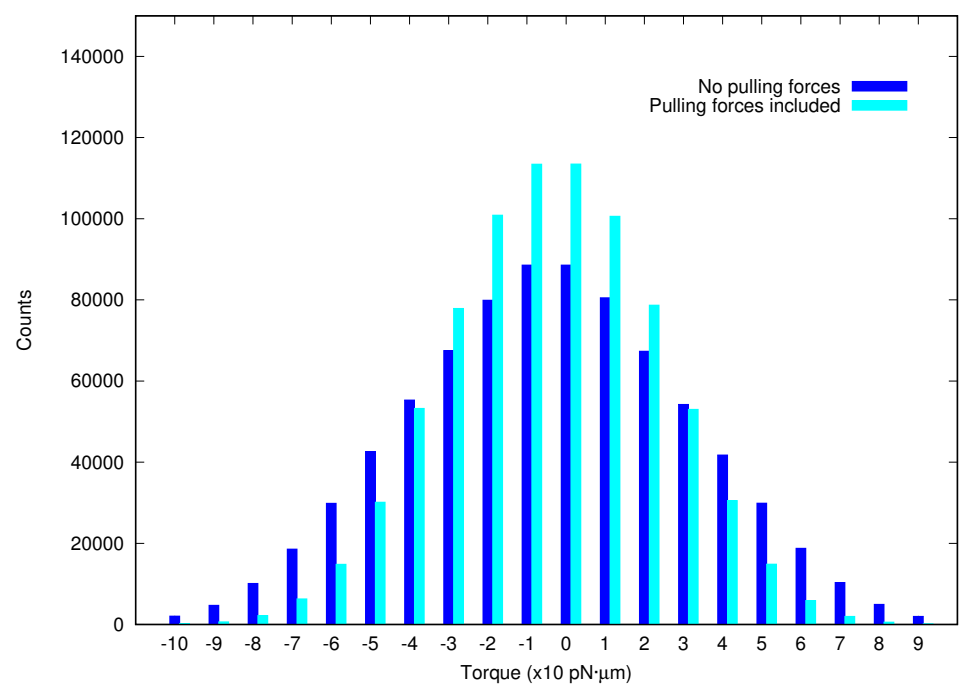

Figure 5.8: Distribution histogram of the torque acting on spindle for the cases with and without pulling forces. Parameters: as in figure 5.5.

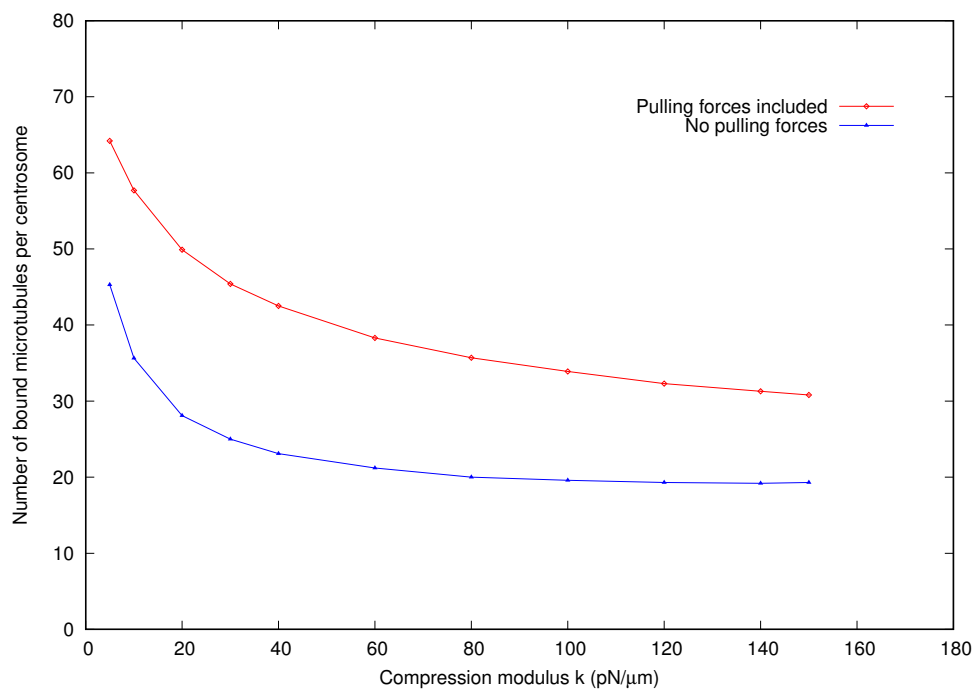

Figure 5.9: Number of bound microtubules as a function of the compression modulus for the cases with and without pulling forces. Parameters: as in figure 5.5 . 


\subsubsection{Transition between spindle orientation states}

Up to this point, our simulation model has been applied to an elliptical confinement for the investigation of the pulling forces impact to the spindle stabilization. Now, we apply the same simulation algorithm to a half-ellipse shape in order to study the influence of the cell shape to the spindle positioning. Taking both pushing and pulling forces acting on the microtubules, we calculate the mean orientation angle of the spindle and the corresponding standard deviation for different values of the short semi-axis a. The simulation results are shown in figure 5.10 .

We can see that there is a transition between two distinct stable states of the spindle orientation, which occurs at the point $2 \mathrm{a} / b=1$. The expected higher fluctuations round the transition point are confirmed by the standard deviation graph.

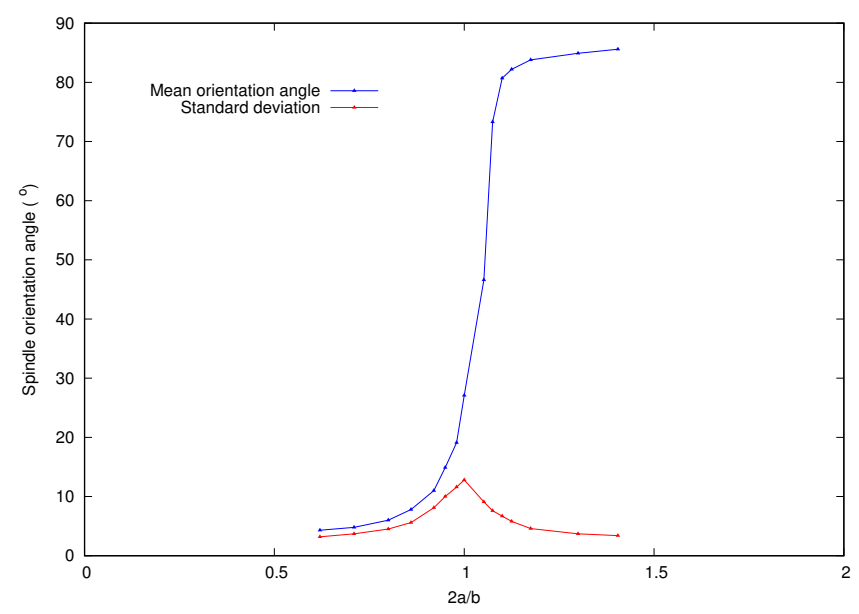

Figure 5.10: Spindle mean orientation angle (blue line) and standard deviation (red line) as functions of the ratio $2 \mathrm{a} / \mathrm{b}$ with both pushing and pulling forces. Parameters: $b=8 \mu \mathrm{m}, L_{\text {spin }}=6 \mu \mathrm{m}, \bar{L}=3.34 \mu \mathrm{m}, W=2 \mu \mathrm{m}$, $\eta_{0}=27.5 \mathrm{~Pa} \cdot \mathrm{s}, \quad k_{b}=0.02 \mathrm{~s}^{-1}, \quad k_{o f f}=0.01 \mathrm{~s}^{-1}, \quad k_{n}=0.03 \mathrm{~s}^{-1}, \quad k=150 \mathrm{pN} / \mu \mathrm{m}$, $\beta \delta=0.60 \mathrm{pN}^{-1}, F_{\text {pull }}=5 \mathrm{pN}$.

To illustrate the differences between the different possible configurations we show snapshots in the steady state of the spindle and the bound microtubules with their 'stored length' for three different values of the short semi-axis close to the critical one, but all other parameters fixed, in figures 


\section{$5.11,5.12,5.13$,}

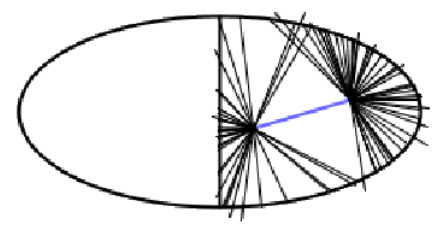

Figure 5.11: Spindle orientation (snapshot) in the steady state with pushing and pulling microtubules for $2 \mathrm{a} / b=0.95$ and $b=12 \mu \mathrm{m}$. We can see the spindle (blue color) and the microtubules (black color) which are bound to the cell boundary (perimeter of half-ellipse) and extend outside it, if they have a 'stored length'. Parameters: $L_{\text {spin }}=6 \mu \mathrm{m}$, $\bar{L}=3.34 \mu \mathrm{m}, W=3 \mu \mathrm{m}, \eta_{0}=27.5 \mathrm{~Pa} \cdot \mathrm{s}, k_{b}=0.02 \mathrm{~s}^{-1}, k_{o f f}=0.01 \mathrm{~s}^{-1}, k_{n}=0.03 \mathrm{~s}^{-1}$, $k=2 \mathrm{pN} / \mu \mathrm{m}, \beta \delta=0.60 \mathrm{pN}^{-1}, F_{\text {pull }}=0.2 \mathrm{pN}$.

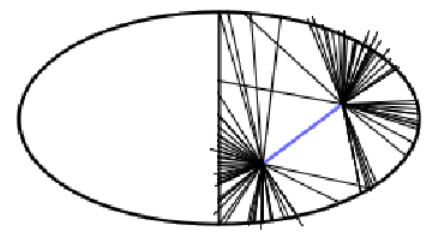

Figure 5.12: Spindle orientation (snapshot) in the steady state with pushing and pulling microtubules for $2 \mathrm{a} / b=1.05$ and $b=12 \mu \mathrm{m}$. Parameters: as in figure 5.11 .

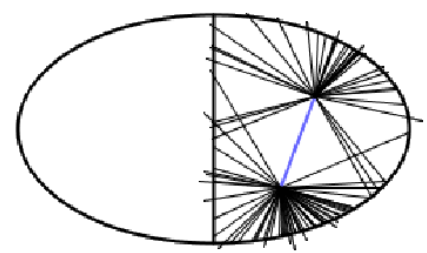

Figure 5.13: Spindle orientation (snapshot) in the steady state with pushing and pulling microtubules for $2 \mathrm{a} / b=1.15$ and $b=12 \mu \mathrm{m}$. Parameters: as in figure 5.11 . 


\subsection{Conclusion}

In this chapter we developed a model for the prediction of the mitotic spindle positioning inside a 2D-confinement. The force production mechanism which was applied is that of the 'stored length' combined with pulling forces acting by dynein motors bound to the boundary. The movement of the spindle was determined by the equation of motion for viscous fluids.

By applying stochastic simulations to an elliptical cell, we calculated the spindle orientation fluctuations with the presence or not of pulling forces. The results revealed the crucial role that pulling forces play in the stabilization of the mitotic spindle. Specifically, when pulling forces are present in the system, the magnitude of the pushing forces is reduced and the number of bound microtubules to the cell boundary increases. In this way, we get lower fluctuations of the spindle orientation angle and, consequently, a higher degree of stabilization.

The simulation model was applied further to a half-ellipse shape for the investigation of the cell shape impact to the spindle positioning. A transition from an almost perpendicular to the short axis spindle orientation state to an almost vertical one was found and the corresponding phase diagram was constructed as a function of the axes ratio.

For simplicity reasons, our model neglected the effect of microtubule sliding on the cell boundary. Also, it didn't take into consideration the interactions between microtubules and chromosomes or the cytoplasmic forces generated by dyneins and cross-linkers of interpolar microtubules. The above interactions are included in a recently developed model 24], which appeared after the work described here was finished. This model, however, unlike the one presented here, does not consider the dynamic nature of the pushing forces acting on microtubules by the cell boundary. In the latter model the pushing forces are strongly length-dependent, and hence geometry-dependent, as they are generated mostly by buckling. A basic difference between the two models concerns the role that pulling forces play. Our model agrees with the model of Laan et al. 22] that pulling forces work towards the direction of a more stable positioning. On the other hand, the previously referred model [24] is compatible with the observations presented in Garzon-Coral et al. [25] which indicate that, at least in $C$. elegans embryo cells, the presence of pulling forces generates instability in the system. Resolving this discrepancy between the models, should be 
pursued in future research.

The dynamic character of the pushing forces is necessary for a realistic representation of the magnitudes of forces acting on the spindle poles. In a future work, however, we need to combine this dynamics with the boundarysliding of microtubules, as they both define the interaction with the cell boundary.

\subsection{Appendix: Relations between the drag coef- ficients}

The Einstein-Smoluchovski relation [67] connects a macroscopic magnitude, which is the absolute temperature $T$, with a microscopic one, which is the diffusion ( $D$ coefficient) of a particle inside a fluid

$$
D=\mu k_{B} T
$$

where $k_{B}$ is the Boltzmann's constant and $\mu$ is the mobility of the particle, which depends on the fluid viscosity and the particle shape. The mobility $\mu$ is the inverse of the drag coefficient $\gamma[68$.

$$
\mu=\frac{1}{\gamma}
$$

From Eq. (5.13) we get

$$
D=\frac{k_{B} T}{\gamma}
$$

We generalize this equation by coupling translation and rotation as follows

$$
[D]=k_{B} T[\gamma]^{-1}
$$

where $[D]$ is the diffusion tensor $(6 \times 6)$ and $[\gamma]$ is the corresponding drag coefficient tensor [69] with

$$
[D]=\left(\begin{array}{ll}
\mathbf{D}_{\mathrm{tt}} & \mathbf{D}_{\mathrm{tr}} \\
\mathbf{D}_{\mathrm{rt}} & \mathbf{D}_{\mathrm{rr}}
\end{array}\right)
$$

and

$$
[\gamma]=\left(\begin{array}{ll}
\gamma_{t t} & \gamma_{t r} \\
\gamma_{r t} & \gamma_{r r}
\end{array}\right)
$$


The elements of these tensors are $(3 \times 3)$ sub-matrices, which correspond to translation (tt), rotation (rr) and translation-rotation coupling (tr). For example, the $\mathbf{D}_{\mathbf{t t}}$ element is

$$
\mathbf{D}_{\mathrm{tt}}=\left(\begin{array}{ccc}
D_{t t}^{x x} & D_{t t}^{x y} & D_{t t}^{x z} \\
D_{t t}^{y x} & D_{t t}^{y y} & D_{t t}^{y z} \\
D_{t t}^{z x} & D_{t t}^{z y} & D_{t t}^{z z}
\end{array}\right)
$$

Then, the translational diffusion coefficient $D_{t}$ is written as [69]

$$
D_{t}=\frac{1}{3} \operatorname{Tr}\left(\mathbf{D}_{\mathbf{t t}}\right)=\frac{1}{3}\left(D_{t t}^{x x}+D_{t t}^{y y}+D_{t t}^{z z}\right)
$$

where $D_{t t}^{z z}=0$, since we regard a $2 \mathrm{D}$-motion on the plane $x y$.

The equation 5.15 holds individually for any of $D_{t t}^{x x}, D_{t t}^{y y}$. So, equation 5.17) becomes

$$
D_{t}=\frac{1}{3}\left(\frac{k_{B} T}{\gamma_{\|}}+\frac{k_{B} T}{\gamma_{\perp}}\right)
$$

since we regard that $x$ and $y$ axes are correspondingly parallel and vertical to the spindle axis.

About the translational drag coefficients $\gamma_{\|}, \gamma_{\perp}$ of rod-like objects with length to width ratio equal or larger than 2 , many analytical solutions have been proposed by different models. All the solutions for the $\gamma_{\|}$drag coefficient have the same basic form [70]

$$
\gamma_{\|}=\frac{2 \pi \eta_{0} L}{\ln (L / W)+C_{\|}}
$$

where $\eta_{0}$ is the viscosity of the cytoplasm, $L$ is the length of the rod-like object, $W$ is its width and $C_{\|}$is a correction factor (numerical constant), which differs among the various solutions.

Correspondingly, the solutions for the $\gamma_{\perp}$ drag coefficient have the following common form [70]

$$
\gamma_{\perp}=\frac{4 \pi \eta_{0} L}{\ln (L / W)+C_{\perp}}
$$

In our model we use the correction factors $C_{\|}, C_{\perp}$ proposed in [71]. Then, 
the calculated values of the two translational drag coefficients are such that we can regard the approximation (5.4).

Following, we get from Eq. 5.18

$$
D_{t}=\frac{1}{2} \frac{k_{B} T}{\gamma_{\|}}
$$

We consider now the rotational diffusion coefficient $D_{r}$ and the corresponding drag coefficient $\gamma_{r}$. Then, by applying Eq.(5.15), we get

$$
D_{r}=\frac{k_{B} T}{\gamma_{r}}
$$

Dividing the last two equations yields

$$
\frac{D_{t}}{D_{r}}=\frac{\gamma_{r}}{2 \gamma_{\|}}
$$

The first part of the above equation can be calculated analytically [72]

$$
\frac{D_{t}}{D_{r}}=\frac{L^{2}}{9} \frac{\ln (L / W)+C_{t}}{\ln (L / W)+C_{r}}
$$

where $C_{t}, C_{r}$ are numerical constants.

Then, from the last two equations we get the approximation (5.5). 


\section{Part III}

\section{Cell polarity}





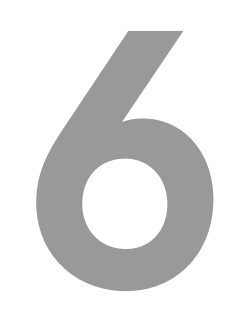

\section{Spherical cell polarity: a minimal model}

The establishment of cell polarity is of high importance to many developmental biological processes. In this chapter, we formulate a minimal model for the spontaneous and persistent generation of polarity in a spherical cell. Our model is based on the positive feedback mechanism that the local density of polarity factors delivered to the membrane by microtubules supports through the stabilization of the microtubules at the membrane. The analytical results are verified by $2 \mathrm{D}$ particle-based simulations. The presented model does not correspond to any known polarity mechanism, but it is based on feasible molecular roles. Hence, it may form the basis of a synthetic biology approach by establishing polarity in a minimal cell-like environment. 


\subsection{Introduction}

The establishment and maintenance of cell polarity, the spatially asymmetric distribution of intracellular components is of crucial importance to many developmental processes in biology, such as anisotropic growth morphologies and asymmetric divisions as precursors to differentiation. The unraveling of the subtle molecular mechanisms underlying these phenomena is an active field of biological research [73]. At the same time, the fundamental nature of this problem has also drawn the attention of biophysicists [74. Building on Turing's seminal work on biological pattern formation in reaction diffusion systems, the so-called Gierer-Meinhardt mechanism of a slow diffusing autocatalytic "activator" competing with a fast diffusing "inhibitor" has developed into a canonical modelling approach towards these questions (for a review see: [75]). The feasibility of such a mechanism was recently demonstrated by the Lim group [76, who designed such networks in silico and implemented them in vivo using a synthetic biology approach.

However, it appears that the cytoskeleton, the dynamic network of protein filaments that performs a host of structural and mechanical roles in all eukaryotic cells, is often implicated in polarity mechanisms [77. A wellknown example is fission yeast where microtubules are involved in depositing polarity factors to the cell ends, which in turn leads to the recruitment of actin nucleators, a key event in establishing polarized growth [78. The question thus arises what role these non-diffusible filaments, whose primary role in interphase cells is to facilitate motor protein-driven linear transport, play in polarity generation. A class of polarity models proposed by the Altschuler-Wu group already implicitly includes the role of cytoskeletal filaments in the form of pre-positioned "patches" on the cell membrane in which the dynamics of a partially membrane bound target molecule is altered [79]. These models do indeed display persistent anisotropic patterns, yet, arguably, do not explain the spontaneous occurrence of symmetrybreaking, as the resultant patterns are predicated on the pre-established position of the patches. The same authors later also considered a single species self-activation model which does generate spontaneous symmetry breaking [80, 81]. However, in the latter model the patterns are not spatially persistent, but fluctuate over time, and disappear when the number of signalling molecules increases, indicating that this is an effect driven by finite particle number noise, rather than a steady state collective phe- 
nomenon. Recently, Freisinger et al. 82] presented the first quantitative model that addresses these shortcomings in the concrete setting of Cdc42 polarization in budding yeast. This model requires two feedback loops to yield a robust axis of polarization, one of which involves an actin-based Cdc42 recycling channel, which itself is reinforced by actin nucleation stimulated by the presence of the active form of Cdc42. In this way the polarity factor Cdc42 can locally stabilize one of its delivery channels to the membrane, effectively spontaneously creating the "patches" of Ref. [79].

Here we show that the latter idea - positive feedback on membrane insertion through stabilisation of transporting structures - is by itself a sufficient mechanism to generate robust cell polarity. We do so by formulating a model that achieves the two desirable features of spontaneous symmetry breaking and steady-state persistence, using a minimal number of components. It is based on the proven ability of microtubules to bind and directionally transport proteins.

Although this model does not correspond to any presently known polarity mechanism in vivo, it is fully based on feasible molecular roles, e.g. motor proteins from the kinesin family are ubiquitous plus-end transporters [83. and a number of proteins is able to stabilize microtubules at membranes [84, 85. At the very least it establishes proof-of-principle and may form the basis of a synthetic biology approach to set up polarity in a minimal celllike environment, such as lipid bilayer-enclosed microvolumes containing purified and/or engineered protein components.

\subsection{The Model}

The key ingredient of the model is that the molecules acting as polarity factors, having been delivered to membrane by dynamical microtubules, stabilize the latter against detaching from the membrane. At the same time, a locally increased concentration of the polarity factors on the membrane, by itself depletes the finite pool of this species present in the cell providing a global inhibitory effect on the propensity of similar stable patches to develop elsewhere. Conceptually this model thus belongs to the generic class of activator-depletion models (for concrete examples see [86, 87] and [75] for a general overview), but distinguishes itself by employing the nondiffusible microtubules as a mediator species. Moreover, it allows an explicit analysis of the conditions under which polarization can occur. The model 
is schematically illustrated in figure 6.1.

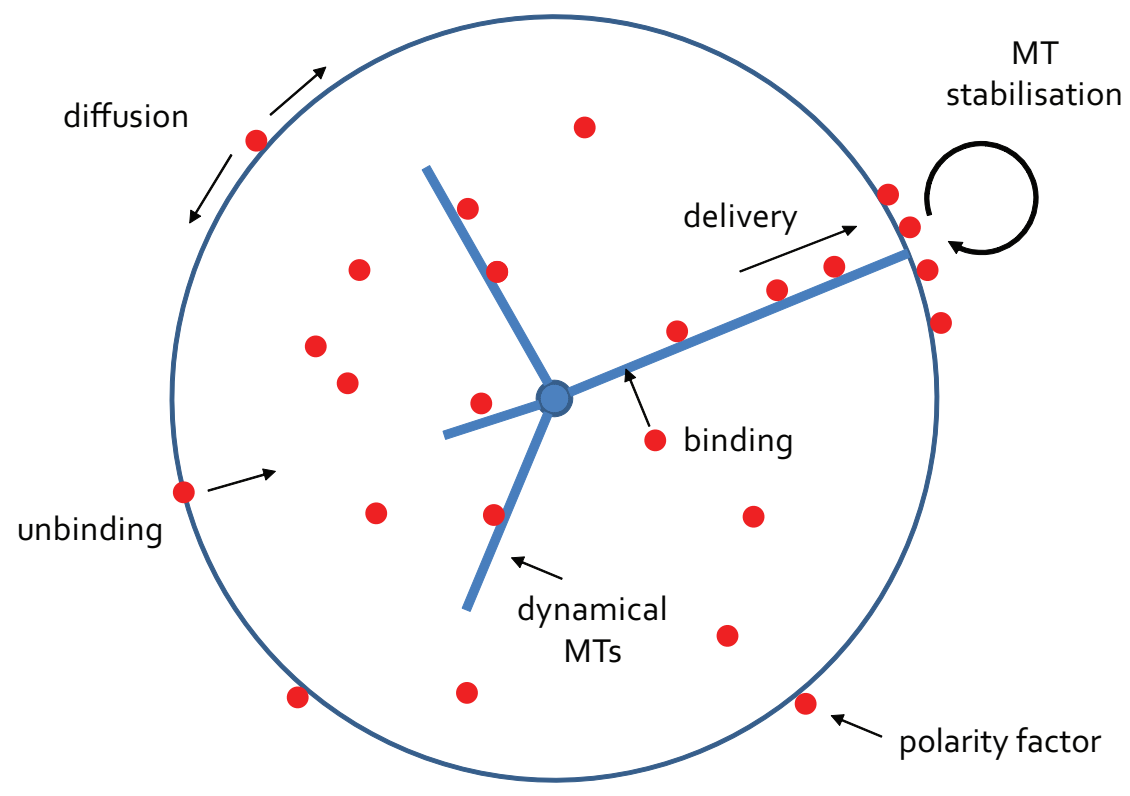

Figure 6.1: Schematic of the model: dynamic microtubules (MTs) transport polarity factors to the membrane. The latter are recycled to the cell interior after diffusing in and unbinding from the membrane. The polarity factors, however, stabilize microtubules against unbinding from the membrane, and thus are able to create local hotspots of polarity factor delivery creating a positive feedback loop leading to spontaneous polarization.

Our setting is a spherically symmetric cell of radius $R$, bounded by a membrane. Microtubules are nucleated from a point-like centrosome in the cell center covered with a constant density $m$ nucleation sites per unit of solid angle, as we have referred in the model of chapter 3 . The unoccupied sites can "fire" with a rate $r_{n}$, creating a new microtubule. The microtubules obey again the standard two-state dynamical instability model [9], with growth speed $v_{+}$, shrinking speed $v_{-}$, catastrophe rate $r_{+}$and rescue rate $r_{-}$. If the microtubules hit the cell boundary they stall, after which they switch to the shrinking state with a rate $r_{u}\left(c_{b}(\hat{\omega}, t)\right)$ which depends on the local concentration $c_{b}(\hat{\omega}, t)$ of the polarity factor in the membrane, where the unit vector $\hat{\omega}$ parameterizes the cell boundary. The dynamical 
equations for the microtubules are then given by

$$
\begin{aligned}
\partial_{t} m_{i}^{+}(l, \hat{\omega}, t)= & -v_{+} \partial_{l} m_{i}^{+}(l, \hat{\omega}, t)+r_{-} m_{i}^{-}(l, \hat{\omega}, t) \\
& -r_{+} m_{i}^{+}(l, \hat{\omega}, t), \\
\partial_{t} m_{i}^{-}(l, \hat{\omega}, t)= & v_{-} \partial_{l} m_{i}^{-}(l, \hat{\omega}, t)-r_{-} m_{i}^{-}(l, \hat{\omega}, t) \\
& +r_{+} m_{i}^{+}(l, \hat{\omega}, t), \\
\partial_{t} m_{0}(\hat{\omega}, t)= & -r_{n} m_{0}(\hat{\omega}, t)+v_{-} m_{i}^{-}(l=0, \hat{\omega}, t), \\
\partial_{t} m_{b}(\hat{\omega}, t)= & -r_{u}\left(c_{b}(\hat{\omega}, t)\right) m_{b}(\hat{\omega}, t)+v_{+} m_{i}^{+}(R, \hat{\omega}, t),
\end{aligned}
$$

where $m_{i}^{+}(l, \hat{\omega}, t)$ and $m_{i}^{-}(l, \hat{\omega}, t)$ are the densities of growing and shrinking microtubules in the direction $\hat{\omega}$ in the cell interior, $m_{b}(\hat{\omega}, t)$ the density of stalled microtubules at the boundary, and $m_{0}(\hat{\omega}, t)$ the density of unoccupied nucleation sites, conveniently considered as density of "dormant" microtubules. These equation are supplemented by the boundary conditions

$$
\begin{aligned}
r_{n} m_{0}(\hat{\omega}, t) & =v_{+} m_{i}^{+}(l=0, \hat{\omega}, t), \\
r_{u}\left(c_{b}(\hat{\omega}, t)\right) m_{b}(\hat{\omega}, t) & =v_{-} m_{i}^{-}(R, \hat{\omega}, t),
\end{aligned}
$$

and the microtubule conservation law

$$
\begin{aligned}
m= & m_{0}(\hat{\omega}, t)+\int_{0}^{R} d l\left\{m_{i}^{+}(l, \hat{\omega}, t)+m_{i}^{-}(l, \hat{\omega}, t)\right\} \\
& +m_{b}(\hat{\omega}, t)
\end{aligned}
$$

The polarity factor can either be adsorbed to the membrane, on which it diffuses with (angular) diffusion constant $D=D_{b} / R^{2}$ and from which it unbinds with rate $k_{u}$, freely diffuse within the interior of the cell, where we assume it diffuses with effective diffusion constant $D_{i}=\infty$ or bind to microtubules. Its conservation law reads

$$
C=C_{f}(t)+C_{m}(t)+\int d \hat{\omega} c_{b}(\hat{\omega}, t)
$$

where $C_{f}$ is the number of free polarity factors in the interior, $C_{m}$ the number bound to microtubules and $c_{b}$ the density on the membrane. We assume that the binding kinetics of the polarity factor to the microtubules is fast with respect to the other processes, so it can be described as an equilibrium, with the density (per unit length) of polarity factors on microtubules 
given by

$$
c_{m}(t)=\frac{C_{m}(t)}{l_{t o t}(t)}=\frac{1}{l_{t o t}(t)+l_{\frac{1}{2}}}\left(C-\int d \hat{\omega} c_{b}(\hat{\omega}, t)\right)
$$

where

$$
\begin{aligned}
l_{t o t}(t)= & \int d \hat{\omega} \int_{0}^{R} d l l\left\{m_{i}^{+}(l, \hat{\omega}, t)+m_{i}^{-}(l, \hat{\omega}, t)\right\} \\
& +R \int d \hat{\omega} m_{b}(\hat{\omega}, t)
\end{aligned}
$$

is the total length of all microtubules in the system, and $l_{\frac{1}{2}}$ is a parameter that sets the affinity of the polarity factors for binding to the microtubules. The polarity factors bound to the microtubules are transported towards the microtubule plus end with speed $v_{m}$, where they are delivered to the membrane, if the microtubule they are bound to is attached to the membrane, or they simply fall off. The time evolution of the polarity factor density on the membrane is thus described by

$$
\partial_{t} c_{b}(\hat{\omega}, t)=D \Delta_{\hat{\omega}} c_{b}(\hat{\omega}, t)-k_{u} c_{b}(\hat{\omega}, t)+v_{m} c_{m}(t) m_{b}(\hat{\omega}, t)
$$

where $\Delta_{\hat{\omega}}$ is the Laplacian on the unit sphere. The final ingredient of the model we need to specify is the dependency of the residence time of the microtubules at the boundary on the local density of polarity factors, which we parametrize as

$$
r_{u}\left(c_{b}\right)=\left(r_{u}(0)-r_{u}(\infty)\right) \sigma\left(\frac{c_{b}}{c_{*}}\right)+r_{u}(\infty)
$$

where $r_{u}(0)>r_{u}(\infty)$ (stabilization), $c_{*}$ sets the relevant density scale and $\sigma(\gamma)$ is a dose-response function that monotonically decreases from a maximal value $\sigma(0)=1$ and vanishes sufficiently fast for large arguments, i.e. $\lim _{\gamma \rightarrow \infty} \gamma \sigma(\gamma)=0$. Our results are generic for a wide class of dose-response functions (see Appendix 6.9.2 to this chapter), but for concreteness sake, we will adopt a standard sigmoidal Hill type function.

\subsection{Steady State Analysis}

In this section we derive the steady state solutions to the microtubule dynamical equations. The steady state equations for the microtubules are 
given by

$$
\begin{aligned}
& 0=-v_{+} \partial_{l} m_{i}^{+}(l, \hat{\omega})+r_{-} m_{i}^{-}(l, \hat{\omega})-r_{+} m_{i}^{+}(l, \hat{\omega}) \\
& 0=v_{-} \partial_{l} m_{i}^{-}(l, \hat{\omega})-r_{-} m_{i}^{-}(l, \hat{\omega})+r_{+} m_{i}^{+}(l, \hat{\omega}) \\
& 0=-r_{n} m_{0}(\hat{\omega})+v_{-} m_{i}^{-}(l=0, \hat{\omega}) \\
& 0=-r_{u}\left(c_{b}(\hat{\omega})\right) m_{b}(\hat{\omega})+v_{+} m_{i}^{+}(R, \hat{\omega})
\end{aligned}
$$

with boundary conditions

$$
\begin{aligned}
r_{n} m_{0}(\hat{\omega}) & =v_{+} m_{i}^{+}(l=0, \hat{\omega}) \\
r_{u}\left(c_{b}(\hat{\omega})\right) m_{b}(\hat{\omega}) & =v_{-} m_{i}^{-}(R, \hat{\omega})
\end{aligned}
$$

Combining Eqs. 6.16 and 6.18) yields

$$
v_{+} m_{i}^{+}(R, \hat{\omega})=v_{-} m_{i}^{-}(R, \hat{\omega})
$$

which in combination with Eqs. (6.13) and 6.14 leads to the well-known [9] balance law

$$
v_{+} m_{i}^{+}(l, \hat{\omega})=v_{-} m_{i}^{-}(l, \hat{\omega})
$$

for all $l \in[0, R]$. This allows the elimination of $m_{i}^{-}(l, \hat{\omega})$ in Eq. 6.13) and its solution with the aid of Eq. 6.15:

$$
\begin{aligned}
& m_{i}^{+}(l, \hat{\omega})=\frac{r_{n}}{v_{+}} m_{0}(\hat{\omega}) e^{-l / \bar{l}} \\
& m_{i}^{-}(l, \hat{\omega})=\frac{r_{n}}{v_{-}} m_{0}(\hat{\omega}) e^{-l / \bar{l}}
\end{aligned}
$$

where

$$
\bar{l}=\left(\frac{r_{c}}{v_{+}}-\frac{r_{r}}{v_{-}}\right)^{-1}
$$

is the mean microtubule length in the absence of boundaries. We can now use the microtubule conservation law

$$
\begin{aligned}
m & =m_{0}(\hat{\omega})+\int_{0}^{R} d l\left\{m_{i}^{+}(l, \hat{\omega})+m_{i}^{-}(l, \hat{\omega})\right\}+m_{b}(\hat{\omega}) \\
& =\left(1+r_{n} \bar{l}\left(\frac{1}{v_{+}}+\frac{1}{v_{-}}\right)\left(1-e^{-R / \bar{l}}\right)\right) m_{0}(\hat{\omega})+m_{b}(\hat{\omega})
\end{aligned}
$$

and the boundary condition Eq. 6.18) which here reads

$$
r_{u}\left(c_{b}(\hat{\omega})\right) m_{b}(\hat{\omega})=v_{-} m_{i}^{-}(R, \hat{\omega})=v_{+} m_{i}^{+}(R, \hat{\omega})=r_{n} m_{0}(\hat{\omega}) e^{-R / \bar{l}}
$$


to determine

$$
\begin{aligned}
& m_{b}(\hat{\omega})=\frac{m r_{n} e^{-R / \bar{l}}}{\left(\left(1+r_{n} \bar{l}\left(\frac{1}{v_{+}}+\frac{1}{v_{-}}\right)\left(1-e^{-R / \bar{l}}\right)\right) r_{u}\left(c_{b}(\hat{\omega})\right)+r_{n} e^{-R / \bar{l}}\right)} \\
& m_{0}(\hat{\omega})=\frac{m r_{u}\left(c_{b}(\hat{\omega})\right)}{\left(\left(1+r_{n} \bar{l}\left(\frac{1}{v_{+}}+\frac{1}{v_{-}}\right)\left(1-e^{-R / \bar{l}}\right)\right) r_{u}\left(c_{b}(\hat{\omega})\right)+r_{n} e^{-R / \bar{l}}\right)}
\end{aligned}
$$

This shows that in steady state all microtubule densities are fully determined by the density of polarity factors at the membrane. For convenience, we now introduce the following dimensionless quantities

$$
\begin{aligned}
& \mu_{i} \equiv r_{n} \bar{l}\left(\frac{1}{v_{+}}+\frac{1}{v_{-}}\right)\left(1-e^{-R / \bar{l}}\right) \\
& \mu_{b} \equiv e^{-R / \bar{l}}
\end{aligned}
$$

which respectively are proportional to the number of microtubules of length $<R$, corresponding to the cell interior, and of length $R$, thus reaching the boundary, in the absence of boundary effects, i.e. dependent only on the microtubule dynamical parameters. We now turn to the calculation of the total length of the microtubules

$$
l_{\text {tot }}(t)=\int d \hat{\omega} \int_{0}^{R} d l l\left\{m_{i}^{+}(l, \hat{\omega}, t)+m_{i}^{-}(l, \hat{\omega}, t)\right\}+R \int d \hat{\omega} m_{b}(\hat{\omega}, t)
$$

which works out as

$$
\begin{aligned}
l_{\text {tot }\left[c_{b}\right]} & =\int d \hat{\omega}\left(\frac{1}{v_{+}}+\frac{1}{v_{-}}\right) r_{n} m_{0}(\hat{\omega}) \int_{0}^{R} d l l e^{-l / \bar{l}}+R \int d \hat{\omega} m_{b}(\hat{\omega}) \\
& =\int d \hat{\omega} \frac{m\left(\frac{1}{v_{+}}+\frac{1}{v_{-}}\right) r_{n} \bar{l}\left(\bar{l}-e^{-R / \bar{l}}(\bar{l}+R)\right) r_{u}\left(c_{b}(\hat{\omega})\right)+R M r_{n} e^{-R / \bar{l}}}{\left(\left(1+r_{n} \bar{l}\left(\frac{1}{v_{+}}+\frac{1}{v_{-}}\right)\left(1-e^{-R / \bar{l}}\right)\right) r_{u}\left(c_{b}(\hat{\omega})\right)+r_{n} e^{-R / \bar{l}}\right)} \\
& =m \bar{l} \int d \hat{\omega} \frac{r_{n} \bar{l}\left(\frac{1}{v_{+}}+\frac{1}{v_{-}}\right)\left(1-e^{-R / \bar{l}}\left(1+\frac{R}{l}\right)\right) r_{u}\left(c_{b}(\hat{\omega})\right)+\frac{R}{l} r_{n} e^{-R / \bar{l}}}{\left(\left(1+r_{n} \bar{l}\left(\frac{1}{v_{+}}+\frac{1}{v_{-}}\right)\left(1-e^{-R / \bar{l}}\right)\right) r_{u}\left(c_{b}(\hat{\omega})\right)+r_{n} e^{-R / \bar{l}}\right)}
\end{aligned}
$$


We introduce the dimensionless quantities

$$
\begin{aligned}
& \lambda_{i} \equiv r_{n} \bar{l}\left(\frac{1}{v_{+}}+\frac{1}{v_{-}}\right)\left(1-e^{-R / \bar{l}}\left(1+\frac{R}{\bar{l}}\right)\right) \\
& \lambda_{b} \equiv \frac{R}{\bar{l}} e^{-R / \bar{l}}
\end{aligned}
$$

proportional to the mean length stored in microtubules of length $<R$ and length equal to $R$ respectively, again in the absence of boundary effects. These definitions allow us to concisely write

$$
l_{\text {tot }}\left[c_{b}\right]=m \bar{l} \int d \hat{\omega} \frac{\lambda_{i} r_{u}\left(c_{b}(\hat{\omega})\right)+\lambda_{b} r_{n}}{\left(1+\mu_{i}\right) r_{u}\left(c_{b}(\hat{\omega})\right)+\mu_{b} r_{n}}
$$

and

$$
m_{b}(\hat{\omega})=m \frac{r_{n} \mu_{b}}{\left(1+\mu_{i}\right) r_{u}\left(c_{b}(\hat{\omega})\right)+\mu_{b} r_{n}}
$$

So, in steady state both the density $c_{m}$ of polarity factors on the microtubules and the density $m_{b}(\hat{\omega})$ of microtubules at the boundary functionally depend on the density $c_{b}(\hat{\omega})$ of polarity factors in the membrane. This allows us to formulate a single autonomous equation for the latter density

$$
D \Delta_{\hat{\omega}} c_{b}(\hat{\omega})-k_{u} c_{b}(\hat{\omega})+K_{b}\left[c_{b}\right](\hat{\omega})=0
$$

where, combining Eq. (6.9) and the steady state solutions, the effective binding rate $K_{b}$ of polarity factors to membrane is given by

$$
\begin{aligned}
K_{b}\left[c_{b}\right](\hat{\omega}) \equiv v_{m} c_{m} m_{b}(\hat{\omega})= & \frac{m v_{m}}{l_{\text {tot }}\left[c_{b}\right]+l_{\frac{1}{2}}}\left(C-\int d \hat{\omega}^{\prime} c_{b}\left(\hat{\omega}^{\prime}\right)\right) \times \\
& \frac{\mu_{b} r_{n}}{\left(1+\mu_{i}\right) r_{u}\left(c_{b}(\hat{\omega})\right)+\mu_{b} r_{n}}
\end{aligned}
$$

Note that since both $l_{\text {tot }}$ and $c_{b}$ are proportional to the number density $m$ of microtubules, a scaling of $l_{\frac{1}{2}}$ with $m$ will leave the effective binding rate invariant. A decrease of $m$ can therefore be exactly compensated by an increased binding affinity of the polarity factors to the microtubules.

Clearly, Eq. 6.36 admits an isotropic solution $\bar{c}_{b}(C)$ for any value of the number of polarity factors. For our purposes it suffices to establish that $\bar{c}_{b}(C)$ is monotonically increasing with $C$ and is asymptotically linear, $\bar{c}_{b}(C) \propto C, C \rightarrow \infty$ (neglecting saturation of binding), so that $\bar{c}_{b}(C)$ can take on any positive value (see Appendix 6.9.1 to this chapter). 


\subsection{Bifurcation Analysis Results}

We now ask whether Eq. (6.36) can also support an anisotropic solution. To that end we adopt a standard bifurcation approach and consider the first order response of the isotropic solution to a perturbation of the type $c_{b}(C)=\bar{c}_{b}(C)+\varepsilon c_{b}^{(1)}(\hat{\omega})$ where $\varepsilon \ll 1$. This leads to the Helmholtz equation

$$
\frac{D}{k_{u}} \Delta_{\hat{\omega}} c_{b}^{(1)}(\hat{\omega})+\Omega^{2}\left(\bar{c}_{b} / c_{*}\right) c_{b}^{(1)}(\hat{\omega})=0
$$

where

$$
\Omega^{2}\left(\bar{c}_{b} / c_{*}\right)=-\left(1+\frac{\bar{c}_{b}}{c_{*}} \frac{\frac{d \sigma}{d \gamma}\left(\bar{c}_{b} / c_{*}\right)}{\sigma\left(\bar{c}_{b} / c_{*}\right)+\eta}\right)
$$

which identifies the parameter

$$
\eta \equiv\left(\frac{r_{u}(\infty)}{r_{u}(0)}+\frac{\mu_{b} r_{n}}{\left(1+\mu_{i}\right) r_{u}(0)}\right) /\left(1-\frac{r_{u}(\infty)}{r_{u}(0)}\right)>0
$$

As expected, the relevant dimensionless scale for the bound polarity factor density is given by the ratio $\gamma \equiv \bar{c}_{b} / c_{*}$, which, in view of its monotonic dependence on $C$, we henceforth adopt as the parameter that governs the availability of polarity factors.

The bifurcation equation (6.38) has spherical harmonics $Y_{n}^{m}(\hat{\omega})$ as solutions provided $\Omega^{2}(\gamma)=n(n+1) D / k_{u}$. Given that $\Omega^{2}(0)=-1$ and $\lim _{\gamma \rightarrow \infty} \Omega^{2}(\gamma)=-1$, we thus first need to establish whether conditions exist for which $\Omega^{2}(\gamma)>0$ for some values of the surface density $\gamma$. If this is the case, a suitably small value of the parameter $\delta \equiv D / k_{u}$, which can be interpreted as the square of the mean angular displacement before unbinding, will always make the unipolar $(n=1)$ solution $Y_{1}^{0}(\hat{\omega}) \propto \cos \theta$ accessible. The explicit form of the dose-response function we adopt is

$$
\sigma(\gamma)=\frac{1}{1+\gamma^{p}}, \quad p>1
$$

which introduces the Hill coefficient $p$ as our final parameter . For this choice

$$
\Omega^{2}(\gamma)=\frac{p \gamma^{p}}{\left(\gamma^{p}+1\right)\left(\eta+\eta \gamma^{p}+1\right)}-1
$$

and we see that $\Omega^{2}(\gamma)=0$ has two real solutions whenever the following criterion is met

$$
\eta<\eta_{\max }=(p-1)^{2} / 4 p
$$


The parameter $\eta$ can be made arbitrarily small by both increasing the residence time of the microtubules at the boundary, i.e. reducing $r_{u}(\infty)$, and simultaneously decreasing the probability of microtubules reaching the boundary, which through the expression $\mu_{b}=\exp (-R / \bar{l})$ is simply governed by the mean length of the microtubules. As argued above, polarization is possible when $\Omega^{2}(\gamma) \geq 2 \delta$. Since $\max \Omega^{2}(\gamma)=p-1-$ $2 p(\sqrt{\eta(1+\eta)}-\eta) \leq p-1$, we find that polarization can only occur for $\delta<\delta_{\max }=1 / 2(p-1)$. Finally, explicitly solving $\Omega^{2}(\gamma)=0$ in the limit $\eta \downarrow 0$, yields an absolute lower bound on the number of polarity factors necessary, which is given by $\gamma_{\min }=(p-1)^{-1 / p}$. The full phase diagram in terms of the three parameters $\eta, \gamma$ and $\delta$ is presented in figure 6.2 .

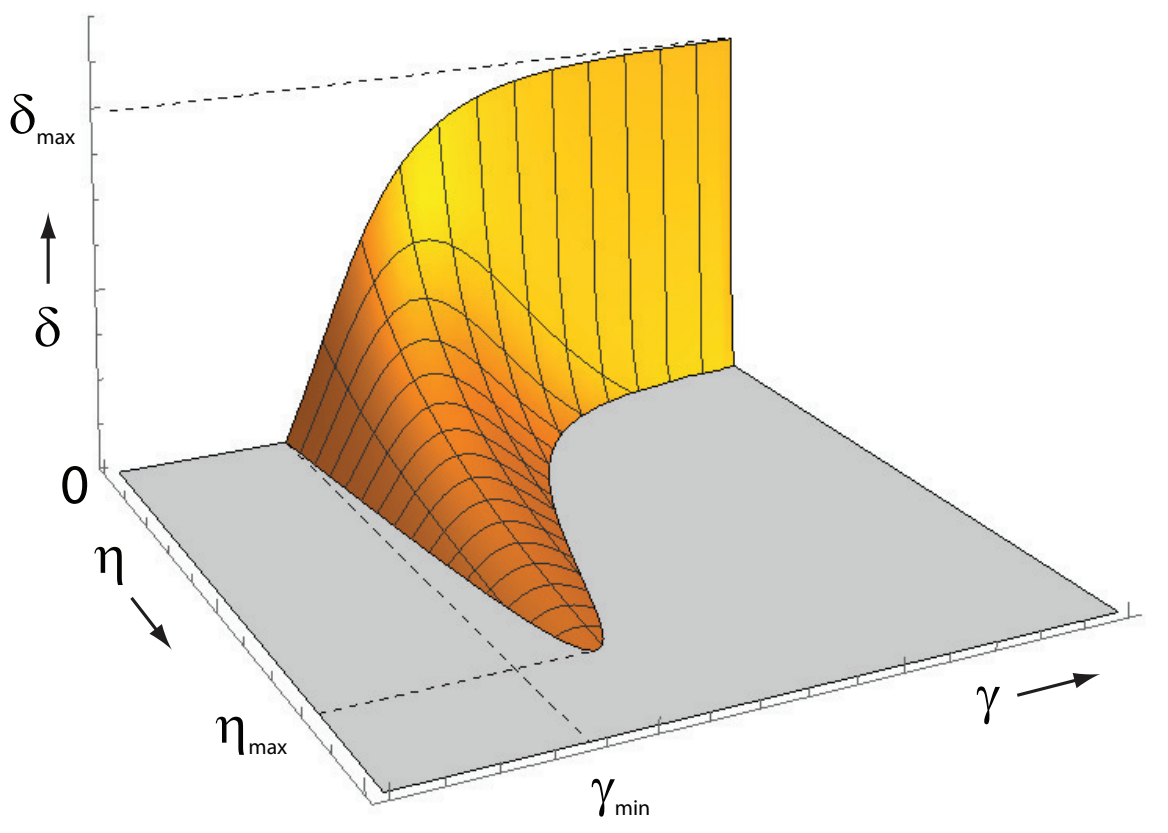

Figure 6.2: Phase diagram of our model for a fixed value of the Hill coefficient $p$. This shows that there is a wide region in the values of the parameters $\eta, \gamma$ and $\delta$, below the depicted boundary surface, for which the polarized state is stable.

On the basis of these results, we can say that spontaneous polarization 
will occur whenever (i) there is a sufficient number of polarity factors (governed by $\gamma$ ), (ii) their effect on enhancing the microtubule residence time at the membrane is high enough (governed by $\eta$ ), (iii) the density dependence of this enhancement is steep enough (governed by $p$ ), (iv) after insertion the polarity factors unbind from the membrane before influencing other microtubules at farther away locations (governed by $\delta$ ), and finally, (v) arrival of microtubules is rare enough (governed by $\eta$ ) that a locally stable polar patch of polarity factor can be established, which then suppresses the formation of other patches through the inhibitory pool depletion effect that decreases the availability of polarity factors to stabilize microtubules at other locations.

For fixed values of $p, \eta$ and $\delta$ which meet the criteria, a value of $C$ (through its proxy $\gamma$ ) can be found above which spontaneous symmetry breaking to a unipolar steady state occurs. However, as the pool of available polarity factors is increased, inevitably the polarization inducing mechanism breaks down: When the monotonically increasing average density of polarity factors in the membrane rises significantly above $c_{*}$, the lifetime of the membrane-bound microtubules becomes $\simeq r_{u}(\infty)^{-1}$ independent of position, and the system will revert back to the isotropic state. We thus expect that as a function of the number of available polarity factors we can distinguish three regimes: At low values of $\gamma$ there are insufficient polarity factors bound to membrane to activate localized regions of longer-lived microtubules. At high values of $\gamma$, the surfeit of available polarity factors precludes any localized increase of polarity factors to inhibit its accumulation at other locations, and microtubules are equally stabilized everywhere. Only in the intermediate regime, where activation balances inhibition can sustained polarization be achieved. Figure 6.3 graphically illustrates this analysis, which also explains the reentrant behavior evident from the phase diagram, where at finite $\eta$ and suitably small $\delta$ any line parallel to the $\gamma$ axis pierces the ordered region at two locations.

Interestingly, the dimensional dependence of the model is in fact very weak, and essentially only enters through the eigenvalue of the angular laplacian (i.e. $n^{2}$ in $2 \mathrm{D}$ vs. $n(n+1)$ in $3 \mathrm{D}$ ). For simplicity's sake, we therefore chose to provide proof-of-principle of our mechanism by simulations of a 2D stochastic version of our model in which both polarity factors and microtubules are explicitly modelled as particles, but note that achieving polarization in $3 \mathrm{D}$ through this mechanism, be it in silico or in vitro, should 
be equally feasible.

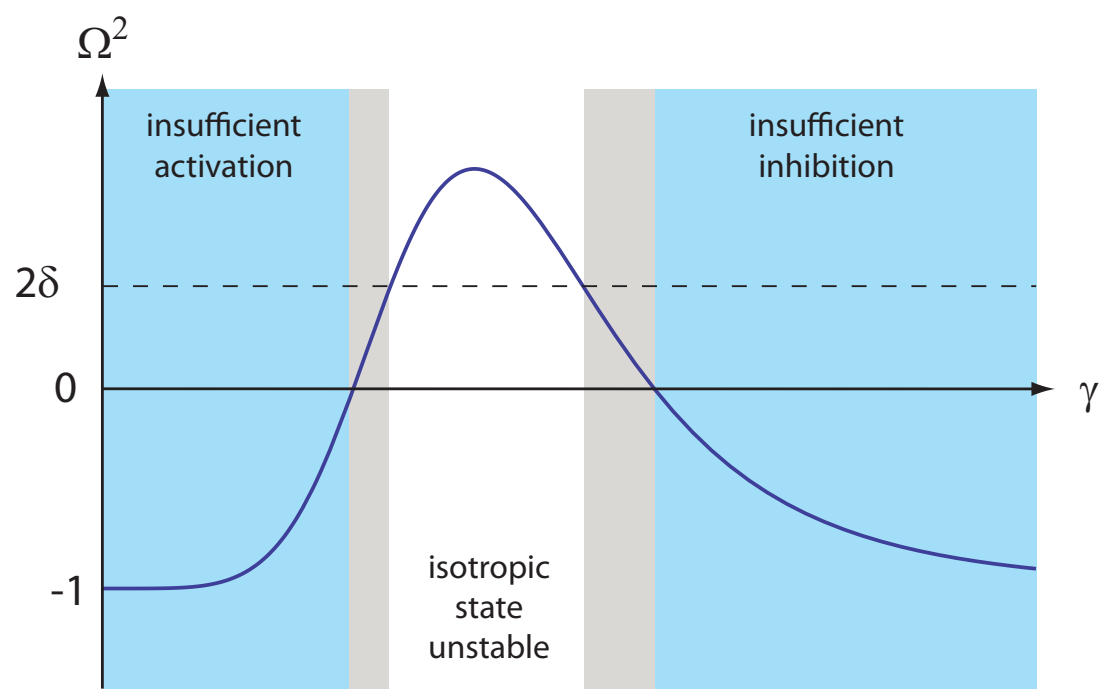

Figure 6.3: The angular wavelength $\Omega^{2}$ as a function of $\gamma$, itself effectively proportional to the total number $C$ of available polarity factors. Spontaneous polarization is possible only in the intermediate regime where the necessary condition $\Omega^{2}>0$ is fulfilled, and is achieved when the sufficient criterion $\Omega^{2}>2 \delta$ is met (white area). In the other two regimes (blue areas) polarization is impossible due to insufficient activation (low $\gamma$ ) or insufficient inhibition (high $\gamma$ ).

\subsection{Simulation Method}

We implement a $2 D$ stochastic simulation in a circular cell geometry with a radius $R$. The control parameter in the simulations is the total number $C$ of polarity factors in the system. The finite time-step in the simulation is chosen to be $\Delta t=0.5 s$. A point-like centrosome in the center of the disc has $M$ nucleation sites that each can nucleate an microtubule in one of $M$ equallyspaced directions. When not connected to an active microtubule, these sites have a nucleation rate $r_{n}$. Active microtubules obey the standard two-state dynamical instability mechanism. When after a time step a microtubule has a length $\geq R$ it is put into the bound state. The boundary of the disc 
is divided to $M$ equal segments, each subtending an angle $\Delta \theta=2 \pi / M$ around one of the nucleation directions, $\theta_{m}=m \Delta \theta, m=0,1,2, \ldots, M-1$. The number of polarity factors in the $m$ 'th segment is denoted by $C_{b}^{m}$. An microtubule in the bound state at angle $\theta_{m}$, can switch to the shrinking state at an instantaneous rate given by $r_{u}\left(c_{m}\right)$ (Eq. (6.12) $)$, where we define the local density of polarity factors as $c_{m}=C_{b}^{m-1}+C_{b}^{m}+C_{b}^{m+1}$ i.e. we regard a neighbourhood also containing the flanking circle segments. This slightly dampens the potentially strong finite number of fluctuations at low values of $C$. At each time step the total length $l_{\text {tot }}$ of active microtubules is evaluated and used to determine the number of microtubule-bound polarity factors $C_{m}$ from the number $C_{i}$ of polarity factors in the cell interior, using the chemical equilibrium approach described earlier.

At the boundary, a polarity factor can either diffuse or unbind thus recycling back into the cell interior. The probability of unbinding from the membrane in a single time step is given by $k_{u} \Delta t$, where $\Delta t$ is the time step and $k_{u}$ is the unbinding rate. Correspondingly, the probability of diffusing on the membrane is given by $1-k_{u} \Delta t$. To determine the angular displacement $\delta \theta$ of a diffusing polarity factor, we sample from the analytical form of the cumulative probability of diffusion on the unit circle

$$
P(\delta \theta)=\frac{\delta \theta}{2 \pi}+\frac{1}{\pi} \sum_{n=1} \frac{e^{-2 n^{2} D \Delta t}}{n} \sin (n \delta \theta),
$$

where we took into account the first 20 terms of the sum and $\Delta t$ is the time step.

Where available, we have used simulation parameters consistent with generic experimental values reported in the literature (see Table 6.1).

The binding affinity of polarity factors to microtubules in our simulations is set by the parameter $l_{1 / 2}$, while in the literature the "binding density" $v$ is used as derived in the McGhee and von Hippel model 994] and defined for one-dimensional lattices as the number of moles of bound ligands per mole of total lattice residue.

To compare the two affinity parameters, we first consider the total number $N$ of available dimers for binding, by introducing the length $l=8 \mathrm{~nm}$ of a single tubulin dimer and taking into account that microtubules have 13 protofilaments and find

$$
N=13 \frac{l_{t o t}}{l}
$$




\begin{tabular}{|c|c|c|c|}
\hline Parameter & Simulation value & Reference value & Reference \\
\hline $\mathrm{R}$ & $3 \mu \mathrm{m}$ & $(2.58 \pm 0.54) \mu \mathrm{m}$ & 88 Budding yeast \\
\hline$v_{+}$ & $0.013,0.018 \mu \mathrm{m} / \mathrm{s}$ & $(0.010-0.033) \mu \mathrm{m} / \mathrm{s}$ & 52 Budding yeast \\
\hline$v_{-}$ & $0.040,0.045 \mu \mathrm{m} / \mathrm{s}$ & $(0.025-0.048) \mu \mathrm{m} / \mathrm{s}$ & [52] Budding yeast \\
\hline$r_{n}$ & $0.05 / \mathrm{s}$ & $(0.007-1.5) / \mathrm{s}$ & $\begin{array}{l}53 \text { Budding Yeast, } \\
89 \text { Kidney epithe- } \\
\text { lium }\end{array}$ \\
\hline$r_{+}$ & $0.0078 / \mathrm{s}$ & $0.0078 / \mathrm{s}$ & 52 Budding Yeast \\
\hline$r_{-}$ & $0.0016 / \mathrm{s}$ & $0.0016 / \mathrm{s}$ & [52] Budding Yeast \\
\hline$v_{m}$ & $0.81 \mu \mathrm{m} / \mathrm{s}$ & $(0.80-0.83) \mu \mathrm{m} / \mathrm{s}$ & 90] Kinesin-1 \\
\hline$k_{u}$ & $0.07 / \mathrm{s}$ & $0.065 / \mathrm{s}$ & 91 Rac \\
\hline $\mathrm{D}$ & $0.02,0.035 \mu \mathrm{m}^{2} / \mathrm{s}$ & $(0.036 \pm 0.017) / \mathrm{s}$ & 79 Yeast \\
\hline$M$ & 1000 & 60 & 92 Xenopus egg \\
\hline $\mathrm{C}$ & $(0.10-0.80) \times 10^{5}$ & $10^{5}$ & 93 Eucaryotes \\
\hline$l_{1 / 2}$ & $15,150 \mu \mathrm{m}$ & $0-\infty$ & $\begin{array}{l}94,95 \text { Dam1 com- } \\
\text { plex }\end{array}$ \\
\hline$(M / 3) c_{*}$ & $20 \times 10^{3}$ & unknown & \\
\hline$r_{u}(0)$ & $0.01 / \mathrm{s}$ & $1 /((90 \pm 35) \mathrm{s})$ & 49 \\
\hline$r_{u}(\infty)$ & $0.001 / \mathrm{s}$ & unknown & \\
\hline$p$ & 5 & unknown & \\
\hline
\end{tabular}

Table 6.1: Model Parameters

The binding density (assuming each dimer can bind a polarity factor) is then simply

$$
\nu=\frac{C_{m}}{N}
$$

On the other hand, Eqs. (6.8) and (6.9) yield

$$
l_{1 / 2}=\frac{C_{f}}{C_{m}} l_{t o t}=\frac{C_{i n t}}{C_{m}} l_{t o t}-l_{t o t},
$$

where $C_{i n t}$ is the total number of polarity factors in the cell interior. Combining the two results, yields the desired relation between the two affinity parameters

$$
l_{1 / 2}=\frac{C_{i n t} l}{13 v}-l_{t o t}
$$




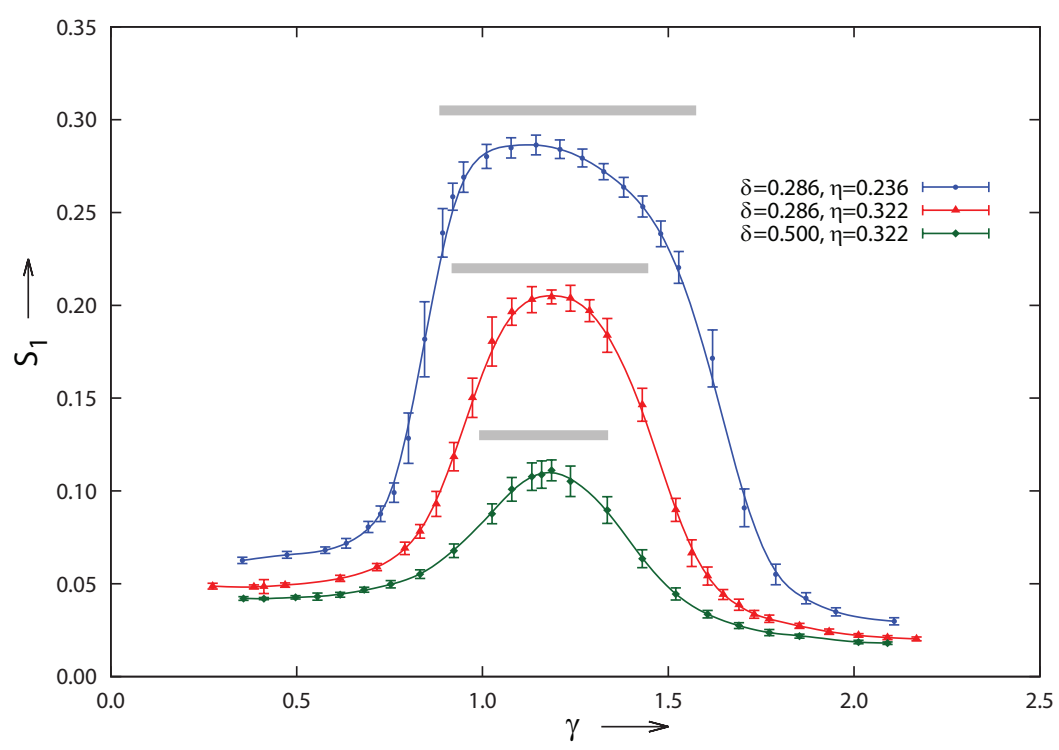

Figure 6.4: The order parameter $S_{1}$ as a function of the average membrane density of polarity factors $\gamma$ as determined in $2 D$ particle-based simulations with $p=5$ and $R=3 \mu \mathrm{m}$. The parameter $\eta$ was tuned by changing the spontaneous catastrophe rate of the microtubules, yielding microtubules of mean length $\bar{l}=1.77 \mu \mathrm{m}$ (top curve) and $\bar{l}=2.54 \mu \mathrm{m}$ (middle and bottom curves).The corresponding theoretical predictions for the polarized regimes are shown as gray bars above each curve. Error bars denote standard errors in the mean from multiple independent runs.

Simulations were run from an initial state with no active microtubules and all polarity factors in the cell interior, until a steady state was reached. To measure the degree of polarization in the steady state we use the order parameter defined by equation (3.47)

$$
S_{1}=\frac{\sqrt{\left(\sum_{m=0}^{M-1} c_{m} \Delta \theta \cos \theta_{m}\right)^{2}+\left(\sum_{m=0}^{M-1} c_{m} \Delta \theta \sin \theta_{m}\right)^{2}}}{\sum_{m=0}^{M-1} c_{m} \Delta \theta}
$$

which has a value of 0 for a perfectly isotropic system and a value of 1 for a fully polarized system, where all polarity factors accumulate in a single bin. To obtain independent samples of the distribution $\left\{C_{b}^{m}\right\}_{m=0, \ldots, M-1}$, observations were spaced by $100 \mathrm{~s}$. Moreover, as expected, the location of 
the maximum of the distribution (if this exists) can slowly drift over the unit circle. To obtain meaningful averages, we therefore corrected for this phase-drift, by extracting the phase through a Fourier analysis and shifting the distribution accordingly. To obtain the averages, roughly 400 samples were taken. At each value of $C$ a number of independent simulations were performed, allowing an error estimate of $S_{1}$ to be obtained (see below). To obtain results as function of the relative membrane density $\gamma=\bar{c}_{b} / c_{*}$, we used Eqs. (6.78) and (6.79) to convert the value of $C$ into the corresponding theoretically predicted value $\gamma$, leading to the graph shown in figure 6.4 of the polar order parameter $S_{1} \equiv\langle\cos \theta\rangle$ describing the angular distribution of polarity factors on the boundary as a function of the mean membrane density $\gamma$ of polarity factors in the cell for three different values of $\eta$ and $\delta$.
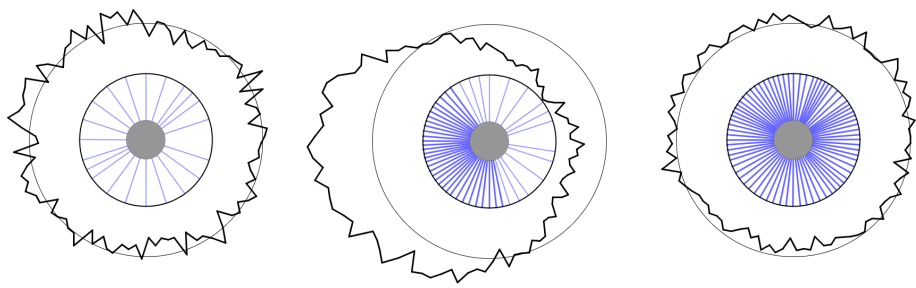

Figure 6.5: Snapshots of the simulation. Left: $\gamma=0.923, C=30000$, center: $\gamma=1.288, C=37000$, and right: $\gamma=1.853, C=50000$. The thick outer contour is a radial histogram of the polarity factor density at the boundary, with the outer circle marking the mean density level. The inner circle represents the cell boundary. For presentation purposes multiple microtubules resident at the boundary are lumped together, the pale blue lines representing a lower density and the dark blue lines a higher density. Also, all microtubules of length $<R$ are not shown, and the dense central area is masked by the gray disk. Values of the remaining parameters: $\eta=0.322, \delta=0.286$ and $p=5$. Results show the predicted sequence of a low polarity factor membrane density isotropic state due to insufficient activation, an intermediate density polarized state, and a high-density state which is again unpolarized due to saturation.

The results show that the observed polarization is both qualitatively 
and quantitatively captured well by the theory, albeit that inevitable finite particle number effects shift the phenomenon to slightly higher values of $\gamma$. Figure 6.5 shows snapshots of the system in the low- $\gamma$ isotropic, the polarized and the high- $\gamma$ saturated regime respectively.

\subsection{Particle Finite Number Effects}

Using our simulation results for $C_{\text {int }}$ and $l_{\text {tot }}$ calculated for $l_{1 / 2}=150 \mu \mathrm{m}$ we find a value of $v \simeq 0.03$, which, comparing to data obtained for the Dam1 complex [95], clearly corresponds to a very low binding-density regime. This suggests that we can readily compensate for a decrease in the number of microtubules to a more realistic value of $\simeq 10^{2}$, by increasing the binding affinity (decreasing $l_{\frac{1}{2}}$ ) to levels which are still feasible. In fact, Eqs. (6.34) and (6.37) show that, everything else remaining equal, a simultaneous scaling of the microtubule density $m$ and $l_{\frac{1}{2}}$ by the same amount, leaves the effective binding rate of polarity factors to membrane invariant, implying that the overall behaviour is identical.

To validate this prediction, we performed simulations with $M=100$ and $l_{\frac{1}{2}}=15 \mu \mathrm{m}$, comparing it to the case $M=1000$ and $l_{1 / 2}=150 \mu m$. The results are shown in figure 6.6 .

Although the two curves are already fairly close, it is obvious that finite particle number effects are more prominent at $M=100$, as evidenced by the significantly higher values of $S_{1}$ in the isotropic phase, be it in the regime of insufficient activation ( factor of 3 larger than at $M=1000$ ) or of insufficient inhibition ( factor of 2 larger than at $M=1000$ ). To estimate the magnitude of these effects we performed additional simulations in which we the total number of polarity factors in the membrane is equal to that of the original simulations, but artificially maintained an isotropic distribution by "homogenizing" the membrane density at each time step. This results in the lower curves in figure 6.6, which are in essence a lower-bound estimate of the finite particle number noise contribution to the observed degree of polarization. Subtracting these noise curves from the full results yields figure 6.7 in which the location of the ordered peak is now seen to fully coincide between the two cases, leaving a reduced amplitude and a slight broadening of the ordered region as the main effects of the reduced number of microtubules. 


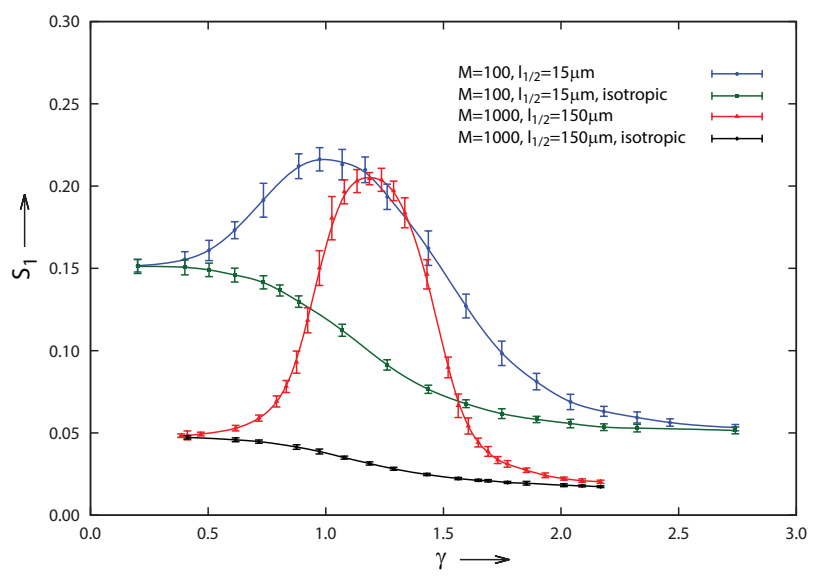

Figure 6.6: The order parameter $S_{1}$ as a function of the average membrane density of polarity factors $\gamma$ for the cases $M=100$ and $M=1000$. For each case two curves are drawn: the "noise curve" obtained by artificially keeping the system in the unpolarized state and the full unconstrained curve. Error bars denote standard errors in the mean from multiple independent runs.

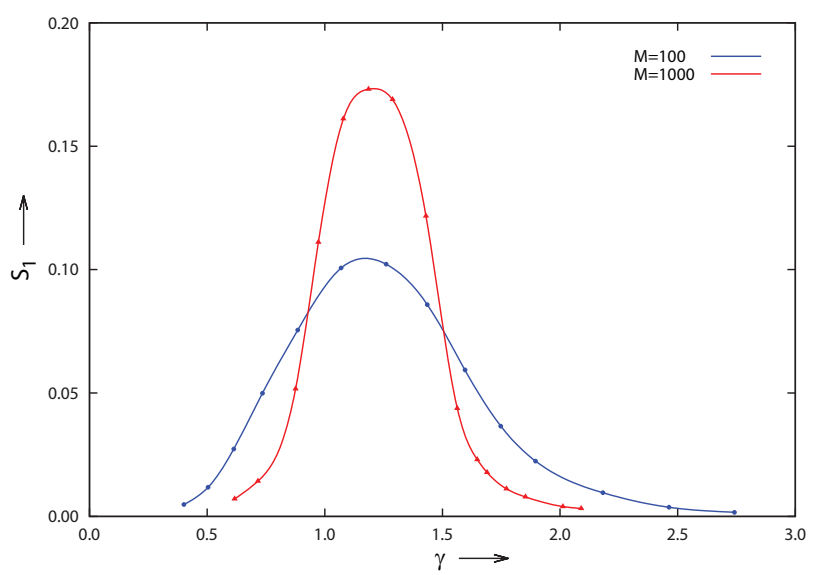

Figure 6.7: The order parameter $S_{1}$ as a function of $\gamma$ for the cases $\mathrm{M}=1000$ (upper curve) and $\mathrm{M}=100$ (lower curve). Each curve is the result of subtracting the noise curve from the full curve (see figure 6.6) of the same case. 


\subsection{Effect of a Finite Tubulin Pool}

In our model we have so far assumed that the amount of tubulin is not a limiting factor. Since, however, we are working in a finite volume it is a reasonable question to ask to what extent our results are robust against possible finite tubulin pool size limitations. Indeed, recent experiments [96] have shown that the size of cytoskeletal structures, such as the mitotic spindle, could well be limited by tubulin availability. Here we address this question, by explicitly modelling the effect a finite pool has on the microtubule dynamics, specifically the growth speed and the nucleation rate. For simplicity, we disregard the effects of the capping of lengths due to cell boundary, focusing on the first order effects.

\subsubsection{Dynamical equations}

We assume the cell contains a finite amount of tubulin, which is represented in terms of a total microtubule length $L_{t o t}$. There are $M$ nucleation sites from which microtubules can be nucleated with a rate given by

$$
r_{n}\left(L_{\text {free }}\right)=r_{n} \frac{L_{\text {free }}^{q}}{L_{\text {free }}^{q}+L_{\frac{1}{2}}}
$$

where $L_{\text {free }}$ is the available free (non-polymerized) tubulin length, $L_{\frac{1}{2}}$ a cross-over parameter, which distinguishes the regime of low availability, in which the rate is strongly limited by the available free pool size, and the regime of high availability, in which the rate ultimately becomes independent of the free pool size. The Hill coefficient $q>1$, describes the potential cooperativity necessary in the nucleation a new microtubule. Once nucleated, microtubules grow with a speed

$$
v_{+}\left(L_{\text {free }}\right)=v_{+} \frac{L_{\text {free }}}{L_{\text {free }}+L_{\frac{1}{2}}},
$$

which at low availability captures the linear dependence of growth on the free tubulin density. The other dynamical parameters $v_{-}, r_{+}$and $r_{-}$are considered to be independent of the available amount of free tubulin. Denoting by $M_{0}(t)$ the total number of free nucleation sites (orientation is unimportant in this context), the dynamics of the system is described by 
the equations

$$
\begin{aligned}
\frac{d}{d t} M_{0}(t) & =-r_{n}\left(L_{\text {free }}(t)\right) M_{0}+v_{-} m_{-}(0, t) \\
\frac{\partial}{\partial t} m_{+}(l, t) & =-v_{+}\left(L_{\text {free }}(t)\right) \frac{\partial}{\partial l} m_{+}(l, t)-r_{+} m_{+}(l, t)+r_{-} m_{-}(l, t) \\
\frac{\partial}{\partial t} m_{-}(l, t) & =v_{-} \frac{\partial}{\partial l} m_{+}(l, t)-r_{+} m_{+}(l, t)+r_{-} m_{-}(l, t) \\
\frac{d}{d t} L_{\text {free }}(t) & =-v_{+}\left(L_{\text {free }}(t)\right) \int_{0}^{\infty} d l m_{+}(l, t)+v_{-} \int_{0}^{\infty} d l m_{-}(l, t)
\end{aligned}
$$

with boundary condition

$$
r_{n}\left(L_{\text {free }}(t)\right) M_{0}=v_{+}\left(L_{\text {free }}(t)\right) m_{+}(0, t)
$$

As a check on these equations we note that they should obey the conservation laws

$$
\begin{aligned}
& M_{0}(t)+\int_{0}^{\infty} d l\left\{m_{+}(l, t)+m_{-}(l, t)\right\}=M \\
& L_{\text {free }}+\int_{0}^{\infty} d l l\left\{m_{+}(l, t)+m_{-}(l, t)\right\}=L_{t o t}
\end{aligned}
$$

Now note that

$$
\begin{aligned}
& \int_{0}^{\infty} d l\left\{\frac{\partial}{\partial t} m_{+}(l, t)+\frac{\partial}{\partial t} m_{-}(l, t)\right\} \\
& =\int_{0}^{\infty} d l\left\{-v_{+}\left(L_{\text {free }}(t)\right) \frac{\partial}{\partial l} m_{+}(l, t)+v_{-} \frac{\partial}{\partial l} m_{+}(l, t)\right\} \\
& =v_{+}\left(L_{\text {free }}(t)\right) m_{+}(0, t)-v_{-} m_{-}(0, t)=-\frac{d}{d t} M_{0}(t)
\end{aligned}
$$

and

$$
\begin{aligned}
& \int_{0}^{\infty} d l l\left\{\frac{\partial}{\partial t} m_{+}(l, t)+\frac{\partial}{\partial t} m_{-}(l, t)\right\} \\
& =\int_{0}^{\infty} d l l\left\{-v_{+}\left(L_{\text {free }}(t)\right) \frac{\partial}{\partial l} m_{+}(l, t)+v_{-} \frac{\partial}{\partial l} m_{+}(l, t)\right\} \\
& =v_{+}\left(L_{\text {free }}(t)\right) \int_{0}^{\infty} d l m_{+}(l, t)-v_{-} \int_{0}^{\infty} d l m_{-}(l, t)=-\frac{d}{d t} L_{\text {free }}(t)
\end{aligned}
$$

where we have assumed $\lim _{l \rightarrow \infty} m_{+}(l, t)=\lim _{l \rightarrow \infty} m_{-}(l, t)=0$. 


\subsubsection{Steady state solution}

We now consider the steady state, for which

$$
\begin{aligned}
-r_{n}\left(L_{\text {free }}\right) M_{0}+v_{-} m_{-}(0) & =0 \\
-v_{+}\left(L_{\text {free }}\right) \frac{\partial}{\partial l} m_{+}(l)-r_{+} m_{+}(l)+r_{-} m_{-}(l) & =0 \\
v_{-} \frac{\partial}{\partial l} m_{+}(l)-r_{+} m_{+}(l)+r_{-} m_{-}(l) & =0 \\
-v_{+}\left(L_{\text {free }}\right) \int_{0}^{\infty} d l m_{+}(l)+v_{-} \int_{0}^{\infty} d l m_{-}(l) & =0
\end{aligned}
$$

with boundary condition

$$
r_{n}\left(L_{\text {free }}\right) M_{0}=v_{+}\left(L_{\text {free }}\right) m_{+}(0)
$$

We note that Eqs. 6.62 and 6.63 lead to

$$
\begin{aligned}
m_{+}(l) & =\frac{m_{0}}{v_{+}\left(L_{\text {free }}\right)} e^{-l / \bar{l}} \\
m_{-}(l) & =\frac{m_{0}}{v_{-}} e^{-l / \bar{l}}
\end{aligned}
$$

where

$$
\bar{l}\left(L_{\text {free }}\right)=\left(\frac{r_{+}}{v_{+}\left(L_{\text {free }}\right)}-\frac{r_{-}}{v_{-}}\right)^{-1}
$$

We can determine $M_{0}, m_{0}$ and $L_{\text {free }}$ through the identities

$$
\begin{aligned}
M & =M_{0}+m_{0}\left(\frac{1}{v_{+}\left(L_{\text {free }}\right)}+\frac{1}{v_{-}}\right) \bar{l}\left(L_{\text {free }}\right) \\
L & =L_{\text {free }}+m_{0}\left(\frac{1}{v_{+}\left(L_{\text {free }}\right)}+\frac{1}{v_{-}}\right) \bar{l}\left(L_{\text {free }}\right)^{2} \\
r_{n}\left(L_{\text {free }}\right) M_{0} & =m_{0}
\end{aligned}
$$


so that

$$
M_{0}=\frac{M}{1+r_{n}\left(L_{\text {free }}\right)\left(\frac{1}{v_{+}\left(L_{\text {free }}\right)}+\frac{1}{v_{-}}\right) \bar{l}\left(L_{\text {free }}\right)}
$$

$$
\begin{aligned}
& L_{\text {free }}= \\
& L-\frac{r_{n}\left(L_{\text {free }}\right) M}{1+r_{n}\left(L_{\text {free }}\right)\left(\frac{1}{v_{+}\left(L_{\text {free }}\right)}+\frac{1}{v_{-}}\right) \bar{l}\left(L_{\text {free }}\right)}\left(\frac{1}{v_{+}\left(L_{\text {free }}\right)}+\frac{1}{v_{-}}\right) \bar{l}\left(L_{\text {free }}\right)^{2} \\
& =L-\frac{r_{n}\left(L_{\text {free }}\right)\left(\frac{1}{v_{+}\left(L_{\text {free }}\right)}+\frac{1}{v_{-}}\right) \bar{l}\left(L_{\text {free }}\right)}{1+r_{n}\left(L_{\text {free }}\right)\left(\frac{1}{v_{+}\left(L_{\text {free }}\right)}+\frac{1}{v_{-}}\right) \bar{l}\left(L_{\text {free }}\right)} M \bar{l}\left(L_{\text {free }}\right)
\end{aligned}
$$

We now take as our length scale $l_{0}=L_{\frac{1}{2}} / M$ and introduce $\lambda=L / L_{\frac{1}{2}}$, $\lambda_{\text {free }}=L_{\text {free }} / L_{\frac{1}{2}}$ and $\bar{\lambda}\left(\lambda_{\text {free }}\right)=\bar{l}\left(L_{\text {free }}\right) / l_{0}$ so that

$$
\lambda_{\text {free }}=\lambda-\frac{r_{n}\left(\lambda_{\text {free }}\right)\left(\frac{1}{v_{+}\left(\lambda_{\text {free }}\right)}+\frac{1}{v_{-}}\right) \bar{\lambda}\left(\lambda_{\text {free }}\right)}{1+r_{n}\left(\lambda_{\text {free }}\right)\left(\frac{1}{v_{+}\left(\lambda_{\text {free }}\right)}+\frac{1}{v_{-}}\right) \bar{\lambda}\left(\lambda_{\text {free }}\right)} \bar{\lambda}\left(\lambda_{\text {free }}\right)
$$

and note that

$$
\frac{L_{\text {free }}}{L_{\text {free }}+L_{\frac{1}{2}}}=\frac{\lambda_{\text {free }}}{\lambda_{\text {free }}+1}
$$

In the Appendix 6.9.3 to this chapter, we prove the following two inequalities:

$$
\begin{aligned}
\bar{\lambda}\left(\lambda_{\text {free }}(\lambda)\right) & \leq \bar{\lambda}\left(\lambda_{\text {free }}(\infty)\right) \\
M-M_{0}\left(\lambda_{\text {free }}(\lambda)\right) & \leq M-M_{0}\left(\lambda_{\text {free }}(\infty)\right)
\end{aligned}
$$

These first inequality implies that, due to the finite tubulin pool, the microtubules are on average shorter than in the saturated case. This decreases the fraction of microtubules reaching the boundary, and hence decreases the parameter $\eta$ (Eq. 6.40), which in turn enhances the propensity 
to polarize. As we have already shown (Eqs. (6.34) and (6.37)), the model can in fact be made robust against the concomitant decrease in the number of active microtubules, implied by the second inequality.

\subsection{Conclusion}

The model discussed here assumed no limits on the availability of tubulin, which could be a concern given the fact that we consider a finite-volume geometry. Having analyzed the first order effects of a limited tubulin pool on the number and length of microtubules using a suitably extended microtubule dynamics, we argue that our model is robust against finite tubulin pool size effects.

In summary, we have presented a minimal, yet feasible, model for spontaneous cell polarization. Although it comprises no less than 13 parameters, the explicit analysis it allows shows that its behavior is in fact only governed by 4 quantities, the source localization parameter $\eta$ (Eq. (6.40), which regulates the efficiency by which microtubules can locally enhance the insertion of polarity factors, the mean angular displacement $\delta=D / k_{u}$ of polarity factors in the membrane, which determines the extent to which polarity factors once inserted remain localized, the Hill parameter $p$, which controls the steepness of the switch that distinguishes stabilized microtubules from non-stabilized microtubules at the membrane, and finally the relative membrane density $\gamma=\bar{c}_{b} / c$, which controls the availability of polarity factors in the membrane to drive the polarization mechanism. We argue that the roles played by these 4 quantities, are universal for a whole class of polarization mechanisms which rely on the autocatalytic enhancement of localized insertion, and as such transcend the specifics of the model presented here. Using techniques currently under active development (see e.g. [97]), it should be possible to implement this type of model in vitro, potentially providing a building block of a minimal synthetic cell. It is of course also interesting to consider how the current model can be coupled to polarized cell growth to further elucidate the biologically highly relevant interplay between cell shape, microtubule organization and polarization. 


\subsection{Appendix}

\subsubsection{Properties of the isotropic solution}

Here we derive the fact that the density of polarity factors on the membrane in the isotropic state increases monotonically with the total number of polarity factors $C$, which is a minimal requirement for the bifurcation to the polarized state to occur.

The steady state equation for the density of polarity factors in the membrane (Eq. 6.36) admits an isotropic solution $c_{b}(\hat{\omega})=\bar{c}_{b}$ which satisfies

$$
-k_{u} \bar{c}_{b}+K_{b}\left(C, \bar{c}_{b}\right)=0
$$

where, making the dependence on the number $C$ of polarity factors explicit,

$$
K_{b}\left(C, \bar{c}_{b}\right)=\frac{m v_{m}}{l_{\text {tot }}\left(\bar{c}_{b}\right)+l_{\frac{1}{2}}}\left(C-4 \pi \bar{c}_{b}\right) \frac{\mu_{b} r_{n}}{\left(1+\mu_{i}\right) r_{u}\left(\bar{c}_{b}\right)+\mu_{b} r_{n}}
$$

with

$$
l_{\text {tot }}\left(\bar{c}_{b}\right)=4 \pi m \bar{l} \frac{\lambda_{i} r_{u}\left(\bar{c}_{b}\right)+\lambda_{b} r_{n}}{\left(1+\mu_{i}\right) r_{u}\left(\bar{c}_{b}\right)+\mu_{b} r_{n}} .
$$

Trivially, $\bar{c}_{b}(C=0)=0$, and an explicit calculation shows that

$$
\frac{d}{d C} \bar{c}_{b}(C=0)=\frac{v_{m} \bar{m}_{b}\left(\bar{c}_{b}=0\right)}{k_{u}\left(l_{\text {tot }}\left(\bar{c}_{b}=0\right)+l_{\frac{1}{2}}\right)+4 \pi v_{m} \bar{m}_{b}\left(\bar{c}_{b}=0\right)} \geq 0
$$

Taking the derivative of Eq. 6.78 with respect to the total number of polarity factors $C$ and rearranging, yields

$$
\frac{d}{d C} \bar{c}_{b}(C)=\frac{\frac{\partial}{\partial C} K_{b}\left(C, \bar{c}_{b}(C)\right)}{k_{u}-\frac{\partial}{\partial \bar{c}_{b}} K_{b}\left(C, \bar{c}_{b}(C)\right)} .
$$

We now note that, from Eq. 6.79

$$
\frac{\partial}{\partial C} K_{b}\left(C, \bar{c}_{b}(C)\right)=\frac{K_{b}\left(C, \bar{c}_{b}(C)\right)}{C-4 \pi \bar{c}_{b}(C)}>0 .
$$

Thus $\frac{d}{d C} \bar{c}_{b}(C)$ can only change sign through a pole (ruled out as $K_{b}\left(C, \bar{c}_{b}\right)$ is bounded) or a cusp (ruled out by smoothness of $K_{b}\left(C, \bar{c}_{b}\right)$ ), showing that $\bar{c}_{b}(C)$ is monotonically increasing. 
Finally, the form of Eq. 6.79 and the asymptotic behaviour of $r_{u}\left(\bar{c}_{b}\right)=$ $r_{u}(\infty)+\mathcal{O}\left(\left(\bar{c}_{b}\right)^{-1-\varepsilon}\right)$ as $\bar{c}_{b} \rightarrow \infty$, which follows from the Ansatz $\lim _{x \rightarrow \infty} x \sigma(x)=0$ on the dose-response function, dictate that $\bar{c}_{b}(C)$ is asymptotically linear. An explicit calculation gives

$$
\bar{c}_{b}(C) \sim \frac{v_{m} \bar{m}_{b}\left(\bar{c}_{b}=\infty\right)}{k_{u}\left(l_{t o t}\left(\bar{c}_{b}=\infty\right)+l_{\frac{1}{2}}\right)+4 \pi v_{m} \bar{m}_{b}\left(\bar{c}_{b}=\infty\right)} C, C \rightarrow \infty,
$$

Together these results imply that $\bar{c}_{b}(C)$ can take on any positive value by suitably choosing $C$.

\subsubsection{Requirements on the dose-response function}

Here we derive the minimal requirements the dose-response function $\sigma\left(\gamma=\bar{c}_{b} / c_{*}\right)$ must meet in order for polarization to be possible.

A necessary requirement for the bifurcation to the polar state to occur is that (see Eq. 6.39)

$$
S(\gamma) \equiv-\gamma \sigma^{\prime}(\gamma)-\sigma(\gamma)=-(\gamma \sigma(\gamma))^{\prime}>\eta>0
$$

This criterion is met (for suitably small $\eta$ ) if $S(\gamma)$ has at least one zero for a finite value of $\gamma$. This, however, is guaranteed as $S(0)=-1$ and by assumption

$$
\lim _{\gamma \rightarrow \infty} \int_{0}^{\gamma} d \gamma^{\prime} S\left(\gamma^{\prime}\right)=-\lim _{\gamma \rightarrow \infty} \gamma \sigma(\gamma)=0
$$

so that $S(\gamma)>0$ for some $\gamma \in(0, \infty)$. If we furthermore assume that $\sigma(\gamma)$ has no more than a single inflection point on $(0, \infty)$, the zero of $S(\gamma)$ is moreover unique. This analysis also shows that the response function need not be sigmoidal. E.g. $\sigma(\gamma)=\exp (-\gamma)$ also works, as $S(\gamma)=$ $(\gamma-1) \exp (-\gamma)$ has an obvious single zero at $\gamma=1$. In this case $\eta_{c}=$ $\max _{\gamma} S(\gamma)=\exp (-2) \simeq 0.135$.

\subsubsection{Proof of the Finite Tubulin Pool Inequalities}

We note that $r_{n}\left(\lambda_{\text {free }}\right),\left(\frac{1}{v_{+}\left(\lambda_{\text {free }}\right)}+\frac{1}{v_{-}}\right)$and $\bar{\lambda}\left(\lambda_{\text {free }}\right)$ are all bounded as $\lambda_{\text {free }} \rightarrow \infty$, so that $\lambda_{\text {free }}(\lambda) \sim \lambda$ as $\lambda \rightarrow \infty$. As $r_{n}(0)$ and $\left(\frac{1}{v_{+}\left(\lambda_{\text {free }}\right)}+\frac{1}{v_{-}}\right) \bar{\lambda}\left(\lambda_{\text {free }}\right) \rightarrow 1 / r_{+}$as $\lambda_{\text {free }} \downarrow 0$, we have $\lambda_{\text {free }}(0)=0$. As 
the second term on the right hand side of Eq. (6.74) is definite positive, we conclude $\lambda_{\text {free }}(\lambda) \leq \lambda$, for all $\lambda$. Consider

$$
\bar{\lambda}(\lambda)=\frac{M}{L_{\frac{1}{2}}}\left(\frac{r_{+}}{v_{+}} \frac{1+\lambda}{\lambda}-\frac{r_{-}}{v_{-}}\right)^{-1}
$$

Thus $\bar{\lambda}(0)=0$ and $\bar{\lambda}(\infty)<\infty$.

Moreover,

$$
\frac{d}{d \lambda} \bar{\lambda}(\lambda)=\frac{M}{L_{\frac{1}{2}}}\left(\frac{r_{+}}{v_{+}} \frac{1+\lambda}{\lambda}-\frac{r_{-}}{v_{-}}\right)^{-2} \frac{r_{+}}{v_{+}} \frac{1}{\lambda^{2}} \geq 0
$$

so that $\bar{\lambda}\left(\lambda_{\text {free }}(\lambda)\right) \leq \bar{\lambda}(\lambda) \leq \bar{\lambda}(\infty)=\bar{\lambda}\left(\lambda_{\text {free }}(\infty)\right)<\infty$. Likewise,

$$
\frac{d}{d \lambda}\left\{\left(\frac{1}{v_{+}(\lambda)}+\frac{1}{v_{-}}\right) \bar{\lambda}(\lambda)\right\}=\frac{M}{L_{1 / 2}} \frac{r_{+}+r_{-}}{\left(\left(r_{-} v_{+}-r_{+} v_{-}\right) \lambda-r_{+} v_{-}\right)^{2}} \geq 0
$$

and

$$
\frac{d}{d \lambda} r_{n}(\lambda)=\frac{q r_{n} \lambda^{q-1}}{\left(\lambda^{q}+1\right)^{2}} \geq 0
$$

So, we can write

$$
\frac{d}{d \lambda}\left\{r_{n}(\lambda)\left(\frac{1}{v_{+}(\lambda)}+\frac{1}{v_{-}}\right) \bar{\lambda}(\lambda)\right\} \geq 0
$$

We also have $r_{n}(\infty)\left(\frac{1}{v_{+}(\infty)}+\frac{1}{v_{-}}\right) \bar{\lambda}(\infty)<\infty$, and so

$$
\begin{gathered}
\frac{M-M_{0}\left(\lambda_{\text {free }}(\lambda)\right)}{M} \leq \frac{M-M_{0}(\lambda)}{M}=\frac{r_{n}(\lambda)\left(\frac{1}{v_{+}(\lambda)}+\frac{1}{v_{-}}\right) \bar{\lambda}(\lambda)}{1+r_{n}(\lambda)\left(\frac{1}{v_{+}(\lambda)}+\frac{1}{v_{-}}\right) \bar{\lambda}(\lambda)} \\
\leq \frac{M-M_{0}(\infty)}{M}=\frac{M-M_{0}\left(\lambda_{\text {free }}(\infty)\right)}{M}
\end{gathered}
$$


Chapter 6. Spherical cell polarity: a minimal model 


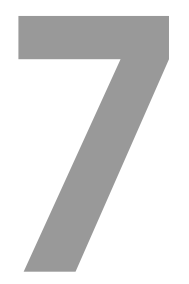

\section{Polarization in non-spherical cells}

We consider the effects of a non-spherical shape on the microtubule-driven polarization mechanism in the setting of an ellipse-shaped model cell. In contrast to the spherical case, two new effects come into play. First, the shape itself will influence the distribution (Chapter 3). Next, the sliding mechanism (Chapter 4) will also contribute to anisotropic ordering. Both these effects appear to promote bipolarity in the distribution of the microtubules. An extensive computational analysis and a theoretical prediction, however, confirm that a unipolar polarization state can still be achieved. 


\subsection{Introduction}

In nature cells are rarely spherical. In fact an ellipsoidal shape is very common [98, 99]. Moreover, many other cell shapes, like the egg shape [100], can be approximated effectively by an ellipsoid. At the same time, there is an active drive to create in vitro reconstructed biomolecular systems in elongated rod-like confinements [101.

It is therefore a logical next step to ask to what extent the microtubulebased cell polarization mechanism introduced in Chapter 6 "survives" in the setting of an ellipsoidal model cell. In Chapter 3 we already showed that shape itself influences the spatial distribution of microtubules nucleated isotropically from a fixed centrosome, due to interactions of the microtubules with the cell boundary. Moreover, in a non-spherical cell the sliding mechanism discussed in Chapter 4 will also contribute to further shape the distribution. In the bilaterally symmetric ellipsoidal cell, both these effects at first sight would bias the distribution to become bipolar.

For reasons of computational convenience also outlined in the previous Chapter, we examine these questions in the $2 \mathrm{D}$ ellipse geometry. We perform stochastic simulations, implementing the polarity mechanism introduced in Chapter 6 , with and without including the sliding mechanism of Chapter 4 . In both cases, we find that for suitable choices of the parameters a unipolar polarized state can still be established. In the case where sliding is included, we encounter the appearance of metastable states indicating the transition to the polarized state is no longer continuous. To probe these effects, we study how the system evolves depending on the choice of pre-biased initial conditions. Finally, to understand why the unipolar state is so robust, we analytically study a simple one dimensional model, which imposes the maximal amount of bi-polar pre-biasing due to the geometry. This rigorously shows that the non-linear polarity mechanism based on diffusive polarity factors transported by the microtubules is strong enough to overcome any bias.

Before addressing these results, the chapter starts with some technical details on the implementation of the simulations. 


\subsection{Simulation details}

\subsubsection{Implementing and validating diffusion}

\subsubsection{The Gaussian approximation}

In the case that we examine, the diffusion of the polarity factors in our model takes place on a closed elliptical boundary. However, in finite time step simulations we can approximate the diffusion process with that of unbounded diffusion in one dimension. To that end, we recall the Gaussian approximation theory [102], which is based on the mapping of the onedimensional diffusion to sampling from a Gaussian step size distribution. The diffusion equation of a particle moving in one dimension is written

$$
c(s, t)=\frac{1}{\sqrt{4 \pi D t}} e^{-\frac{s^{2}}{4 D t}}
$$

where $D$ is the diffusion constant. On the other hand, the Gaussian that is symmetric round zero obeys the equation

$$
f(s)=\frac{1}{\sqrt{2 \pi \sigma^{2}}} e^{-\frac{s^{2}}{2 \sigma^{2}}}
$$

So using the Einstein relation

$$
\sigma^{2}=2 D t
$$

the one-dimensional diffusion is mapped onto a Gaussian. In the simulations the average 'jump' of the diffusing particle is approximated by $\sigma=\sqrt{2 D \Delta t}$, where $\Delta t$ is the time step. If $\Delta t$ is sufficiently small, the mean diffusion jump $\sigma$ becomes much smaller than the circumference $C_{\text {ell }}$ of the ellipse. In this case, the jumps never "feel" the closed shape and the approximation is reasonable.

\subsubsection{Solution of diffusion equation}

Before we proceed with simulations, we develop an analytical method that can validate the simulation results of the Gaussian approximation method. To this end, we regard the diffusion equation on the ellipse boundary

$$
\frac{\partial c(s, t)}{\partial t}=D \frac{\partial^{2} c(s, t)}{\partial s^{2}}
$$


where $c$ is the concentration on the boundary, $s$ is the arc length along the boundary and $t$ is time. At time $t=0$, we regard a single particle at the position $s=s_{0}$. So, we have the initial condition

$$
c(s, 0)=\delta\left(s-s_{0}\right)
$$

Using separation variables, we assume a solution of the form

$$
c(s, t)=U(t) W(s)
$$

Then, by substitution in Eq. (7.4) we get

$$
U^{\prime}(t) W(s)=D U W^{\prime \prime}(s)
$$

and following

$$
\frac{U^{\prime}}{D U}=\frac{W^{\prime \prime}}{W}=-k^{2}
$$

which gives

$$
U(t)=U(0) e^{-k^{2} D t}
$$

The initial condition depends only on $s$, so $U(0)=1$. Setting

$$
k^{2}=\left(\frac{2 \pi m}{C_{\text {ell }}}\right)^{2}
$$

we get the spatial solution

$$
W(s)=\cos \left(\frac{2 \pi m}{C_{\text {ell }}}\left(s-s_{0}\right)\right)
$$

where $C_{\text {ell }}$ is the perimeter of the ellipse and $m$ an integer.

Finishing with the calculation of the probability density function, we expand the initialized by the delta function $c(s, t)$ to a Fourier series

$$
c(s, t)=\frac{1}{C_{\text {ell }}}+\frac{2}{C_{\text {ell }}} \sum_{m=1}^{\infty} e^{-\left(\frac{2 \pi m}{C_{e l l}}\right)^{2} D t} \cos \left(\frac{2 \pi m}{C_{\text {ell }}} s_{0}\right) \cos \left(\frac{2 \pi m}{C_{\text {ell }}} s\right)
$$

For $t \rightarrow \infty$ we see that the probability density of the particle ultimately becomes homogeneously spread out over the circumference, $c(s, \infty)=1 / C_{\text {ell }}$. 


\subsubsection{Validation of diffusion algorithm}

Having extracted the analytical expression $(7.12$ for the probability density function, we proceed with validating the simulation model that is based on the Gaussian approximation method. For efficient sampling from a normal distribution, we use the well-known 'ziggurat' code [103]. We regard an ellipse with short semi-axis $a=2 \mu m$ and long semi-axis $b=4 \mu m$, and we divide its boundary into 400 equal bins. We also take $D=0.035 \mu \mathrm{m}^{2} / \mathrm{s}$ and a small time step $\Delta t=0.5 \mathrm{~s}$. With these choices, the bin length works out as about $0.048 \mu m$ and the mean diffusion jump as $\sigma=0.19 \mu m$, which is about 4 times the bin length. The particles are inserted by a point-source on the boundary with a rate $k_{n}=0.05 s^{-1}$. The simulation results are in full quantitative agreement with the theoretical ones of Eq. 7.12, as it is shown in figure 7.1. In this way, the diffusion algorithm is validated for a small time step. For a complete validation of the algorithm, we proceed with simulations for longer times. For the same set of parameters as before, the simulation results and the analytical ones from Eq. (7.12) are in quantitative agreement, as it is shown in figure 7.2 .

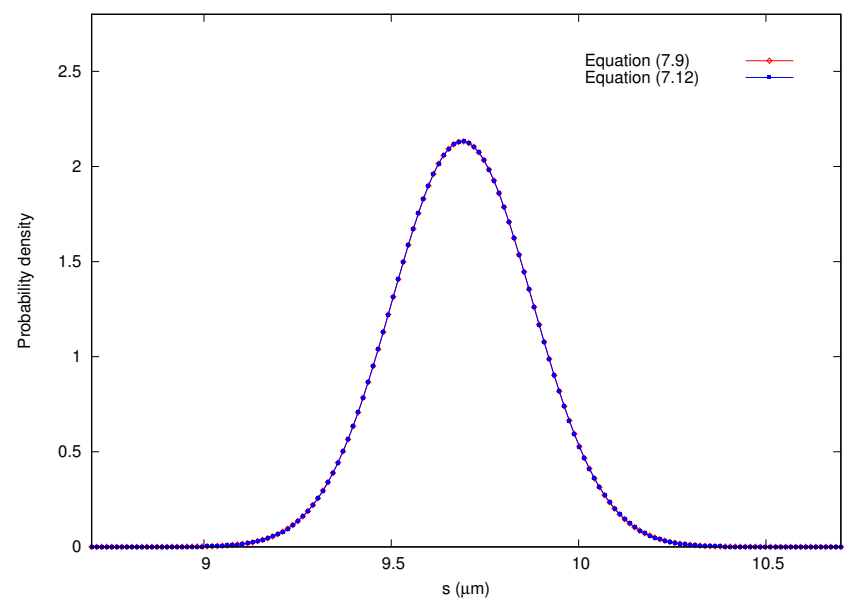

Figure 7.1: Validation of the Gaussian approximation for a small time step $(t=0.5 s)$ of the diffusion process on an elliptical boundary. We can see the high agreement between the probability densities of equations (7.12) and (7.1) for a source of polarity factors positioned at $s=C_{\text {ell }} / 2$. Parameters: $C_{\text {ell }}=19.3788 \mu \mathrm{m}, \mathrm{D}=0.035 \mu \mathrm{m}^{2} \mathrm{~s}^{-1}$. 


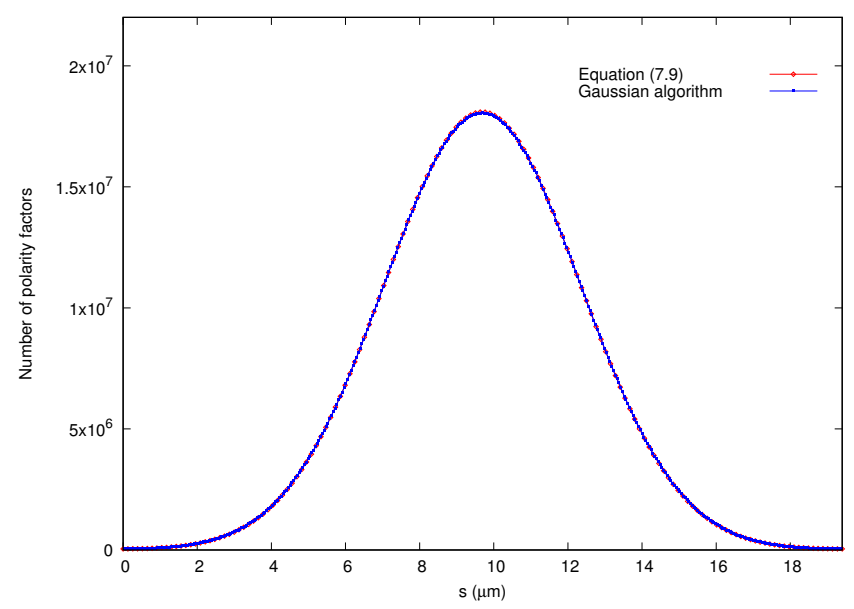

Figure 7.2: Validation of the Gaussian algorithm for longer times. By applying it repeatedly for small time steps $(\Delta t=0.5 \mathrm{~s})$, we recover the resulting distribution of Eq. 7.12 for a total time of $t=100 \mathrm{~s}$. Parameters: as in figure 7.1 .

\subsubsection{Validation of diffusion with source and unbinding}

As we referred to at the beginning of this chapter, we are interested in examining the polarization of elliptical cells that is induced by the spatial organization of microtubules. So, except the diffusion process of the polarity factors, the microtubule nucleation from a centrosome and the unbinding from the cell boundary are also basic processes that we incorporate in our model. In order to validate a simulation algorithm, which includes diffusion, nucleation and unbinding, we again develop an analytical result. To this end, we regard a system with $N$ particles in total, from which $N_{i}$ are distributed over the interior. Particles from the interior are inserted at position $s=0$ with a rate $k_{n}$ per particle per unit time. Within the boundary the particles diffuse with diffusion coefficient $D$ until they unbind with rate $k_{u}$, and are recycled to the interior again. We are looking for the steady-state distribution of the density $c(s)$ of particles in the boundary. The time dependent problem is given by

$$
\frac{\partial}{\partial t} c(s, t)=D \frac{\partial^{2}}{\partial s^{2}} c(s, t)-k_{u} c(s, t)+k_{n} N_{i}(t) \delta(s)
$$


In steady state we find

$$
D \frac{d^{2}}{d s^{2}} c(s)-k_{u} c(s)+k_{n} N_{i} \delta(s)=0
$$

We solve the equation by Fourier analysis, multiplying by $\cos \left(2 \pi m \frac{s}{C_{\text {ell }}}\right)$ (the solution should be symmetric around $s=0$ ) and integrating, yielding

$$
\int_{0}^{C_{e l l}} \cos \left(2 \pi m \frac{s}{C_{e l l}}\right)\left(D \frac{d^{2} c}{d s^{2}}-k_{u} c+k_{n} N_{i} \delta(s)\right) d s=0
$$

By analyzing to terms, we get

$$
\begin{array}{r}
\int_{0}^{C_{e l l}} D \cos \left(2 \pi m \frac{s}{C_{e l l}}\right) \frac{d^{2} c}{d s^{2}} d s-\int_{0}^{C_{e l l}} k_{u} \cos \left(2 \pi m \frac{s}{C_{e l l}}\right) c d s+ \\
\int_{0}^{C_{e l l}} k_{n} N_{i} \delta(s) \cos \left(2 \pi m \frac{s}{C_{e l l}}\right) d s=0
\end{array}
$$

Using the periodicity identity of cosine and sine functions, we get from the last equation

$$
-D\left(\frac{2 \pi m}{C_{e l l}}\right)^{2} \frac{C_{e l l}}{2} c_{m}-k_{u} \frac{C_{e l l}}{2} c_{m}+k_{n} N_{i}=0
$$

where

$$
c_{m}=\frac{2}{C_{\text {ell }}} \int_{0}^{C_{e l l}} \cos \left(2 \pi m \frac{s}{C_{\text {ell }}}\right) c(s) d s
$$

is the m-th Fourier coefficient of the density function $c(s)$. From Eq. 7.17$)$ we take

$$
c_{m}=\frac{k_{n} N_{i}}{\frac{C_{e l l}}{2}\left(k_{u}+D\left(\frac{2 \pi m}{C_{e l l}}\right)^{2}\right)}
$$

So, the Fourier expansion of the probability density function $c(s)$ in steady state becomes

$$
\begin{aligned}
c(s) & =\frac{1}{2} c_{0}+\sum_{0}^{\infty} c_{m} \cos \left(2 \pi m \frac{s}{C_{\text {ell }}}\right) \\
& =\frac{1}{C_{\text {ell }}} \frac{k_{n} N_{i}}{k_{u}}+\frac{2}{C_{\text {ell }}} \sum_{0}^{\infty} \frac{k_{n} N_{i}}{k_{u}+D\left(\frac{2 \pi m}{C_{\text {ell }}}\right)^{2}} \cos \left(2 \pi m \frac{s}{C_{\text {ell }}}\right)
\end{aligned}
$$


Now, we can proceed to simulate the system that includes nucleation, diffusion and unbinding, which we analyzed previously. The distribution of particles on the boundary calculated by the simulations for this system, are in a satisfactory agreement with the distribution predicted by equation (7.20), as we can see in figure 7.3 .

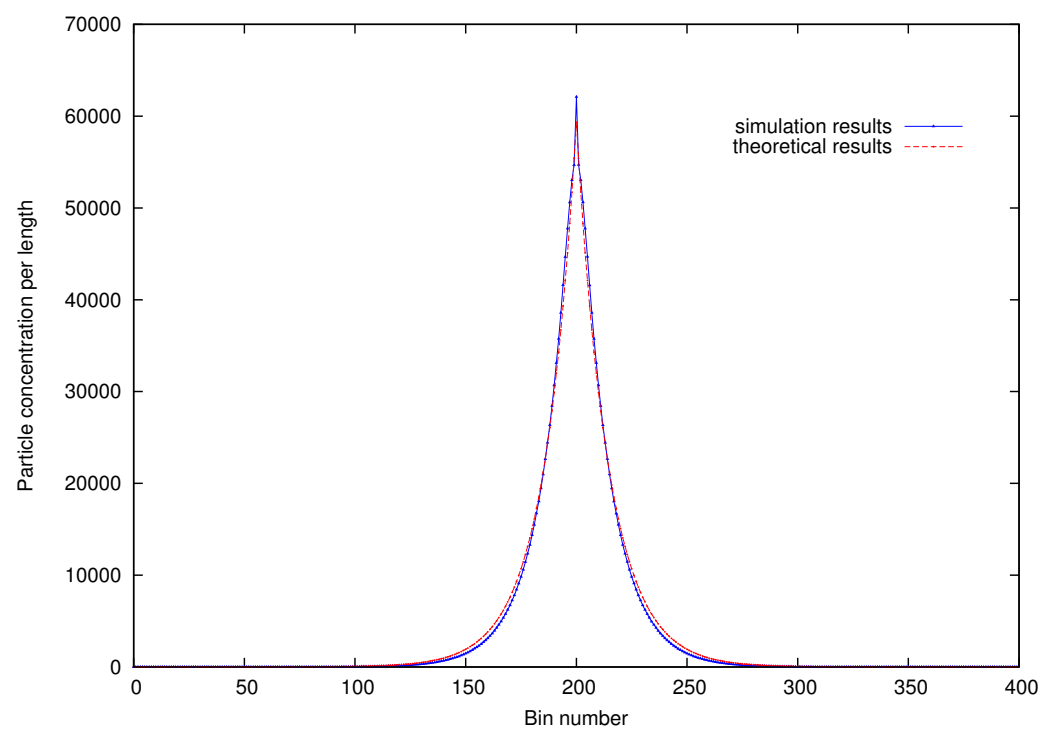

Figure 7.3: Theoretical and simulation results for a system with nucleation, diffusion and unbinding. There is a satisfactory agreement between the simulation results and the distribution defined by the probability density of Eq. 7.20). Parameters: $D=0.035 \mu \mathrm{m}^{2} \mathrm{~s}^{-1}, \Delta t=0.5 \mathrm{~s}, k_{u}=0.07 \mathrm{~s}^{-1}$, $k_{n}=0.05 s^{-1}, N=200000, a=2 \mu m, b=4 \mu m$.

\subsubsection{Force production in presence of polarization factors}

In the following, we are going to study the effect on polarization when microtubule ends can both slide along the boundary of an ellipse and transfer polarity factors to it. For that purpose, we use the boundary-sliding model that was developed in Chapter 4. This model describes the behavior of the sliding microtubules on the boundary, without regarding the presence and impact of any polarity factors. Here, the algorithm for the diffusion of particles on the boundary and the unbinding from it, which we vali- 
dated earlier analytically, is added to that model. Furthermore, this model does not yet include the polarity mechanism that is based on the residence time of the microtubules on the boundary. As we described in Chapter 6, this mechanism relies on the change of the catastrophe rate of the bound microtubules, depending on the concentration of the polarity factors at each boundary site. To develop a simulation model for the investigation of polarity caused by sliding microtubules across an elliptical boundary, we have to connect the force-production mechanism to the polarity-generation mechanism.

We apply the following procedure: First, we recall the equation of the force-production mechanism that gives the mean waiting time $\left\langle\tau_{c}\right\rangle$ until a microtubule undergoes catastrophe [60]:

$$
\left\langle\tau_{c}\right\rangle=\frac{1}{r_{+}} \sqrt{\pi} \sqrt{A} e^{A} \operatorname{erfc}(\sqrt{A})
$$

where

$$
A=\frac{r_{+}}{2 \beta \delta k v_{+}}
$$

erfc is the complementary error function

$$
\operatorname{erf} z=\frac{2}{\sqrt{\pi}} \int_{z}^{\infty} e^{-t^{2}} d t
$$

and $r_{+}, v_{+}$are the force-free values of the catastrophe rate and the growth speed respectively. On the other hand, in the polarity mechanism which we introduced in Chapter 6 the catastrophe rate of bound microtubules, which is equivalent to the unbinding rate $r_{u}\left(c_{b}\right)$, is a function of the concentration $c_{b}$ of polarity factors on boundary sites according to Eq. 6.12). In order to connect the two mechanisms, we require

$$
\left\langle\tau_{c}\right\rangle\left(c_{b}\right) \equiv \frac{1}{r_{u}\left(c_{b}\right)}=\frac{1}{r_{+}\left(c_{b}\right)} \sqrt{\pi} \sqrt{A\left(r_{+}\left(c_{b}\right)\right)} e^{A\left(r_{+}\left(c_{b}\right)\right)} \operatorname{erfc}\left(\sqrt{A\left(r_{+}\left(c_{b}\right)\right)}\right),
$$

i.e. we choose the feedback mechanism to modulate the force-free catastrophe rate.

In the absence of polarity factors, we regard the same values of the growth speed $v_{+}=0.018 \mu \mathrm{m} / \mathrm{s}$, the bare catastrophe rate $r_{+}=0.0078 \mathrm{~s}^{-1}$ and the unbinding rate $r_{u}(0)=0.01 s^{-1}$ (i.e. $\left\langle\tau_{c}\right\rangle\left(c_{b}\right)=100 s$ ) as in Chapter 6. We then use Eq. 7.21 to solve for the compression modulus, yielding 
$k=0.3 p N / \mu m$. With these values in place, we solve Eq. (7.24) for $r_{+}\left(c_{b}\right)$ over a range of $c_{b}$ values, tabulating the results. These tabulated results are used in the simulation to determine, together with the values for the force produced, the instantaneous catastrophe rate for any microtubule at the boundary. Fig. 7.4 shows the calculated catastrophe rate as function of the density of polarity factors at the membrane.

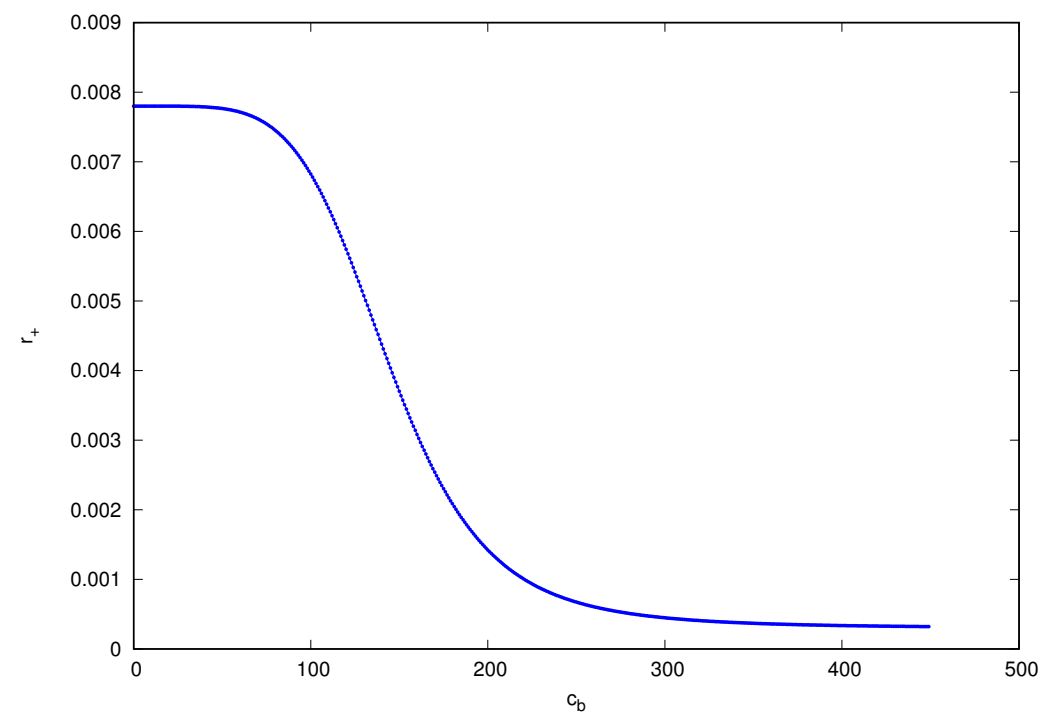

Figure 7.4: Force-free catastrophe rate $r_{+}\left(\right.$in $\left.s^{-1}\right)$ as a function of the polarity factors density $c_{b}$ (per bin) at the cell boundary (ellipse with $a=2 \mu \mathrm{m}$, $\mathrm{b}=4 \mu \mathrm{m})$.

\subsubsection{Order parameters}

The appropriate independent variable for describing the density of polarity factors on the boundary of the ellipse is the arc length at each point of the boundary, already used in the theoretical solution 27.20), measured from a length origin, conveniently located on the major axis at one of the poles. This means we also need to map the nucleation angles to arc lengths on the ellipse boundary. To that end, we choose to transform the nucleation angles $\phi$ of polar coordinates to angles $\nu$ of elliptic coordinates, following the procedure that we developed in Chapter 3. 
We must also consider the proper analog of the angle-based order parameter used in Chapter 6. Furthermore, we have to consider not one but two order parameters, since the elliptical geometry can give also a bipolar state, apart from the unipolar one. These can be constructed by replacing the angular argument in the trigonometric functions $\cos$ and $\sin$ by $2 \pi s / C_{\text {ell }}$ for the unipolar order parameter and $4 \pi s / C_{\text {ell }}$ for the bipolar one in the definition equations (3.47), 3.46).

So, the scalar unipolar order parameter $S_{1}$ is defined as

$$
S_{1}=\frac{\sqrt{\left(\sum_{m=0}^{M-1} C_{b}^{m} \cos \left(2 \pi s_{m} / C_{e l l}\right)\right)^{2}+\left(\sum_{m=0}^{M-1} C_{b}^{m} \sin \left(2 \pi s_{m} / C_{e l l}\right)\right)^{2}}}{\sum_{m=0}^{M-1} C_{b}^{m}}
$$

where $s_{m}=(m+1) \Delta s$ is the coordinate of the m-th bin, $M$ is the number of bins and $C_{b}$ is the number of the polarity factors bound on the $m$-th bin.

The bipolar order parameter $S_{2}$ is defined as the algebraic mean value of $\cos \left(4 \pi s / C_{\text {ell }}\right)$

$$
S_{2}=\frac{\sum_{m=0}^{M-1} C_{b}^{m} \cos \left(4 \pi s_{m} / C_{e l l}\right)}{\sum_{m=0}^{M-1} C_{b}^{m}}
$$

The positive values of $S_{2}$ imply a longitudinal bipolar phase, while negative values a transverse bipolar phase.

\subsection{Results}

\subsubsection{Polarization in the absence of sliding}

We first consider a model that is essentially the same as the microtubulebased minimal model for spherical polarity developed in previous chapter. The only difference concerns the cell shape, which now is elliptical (see figure 7.5 . 


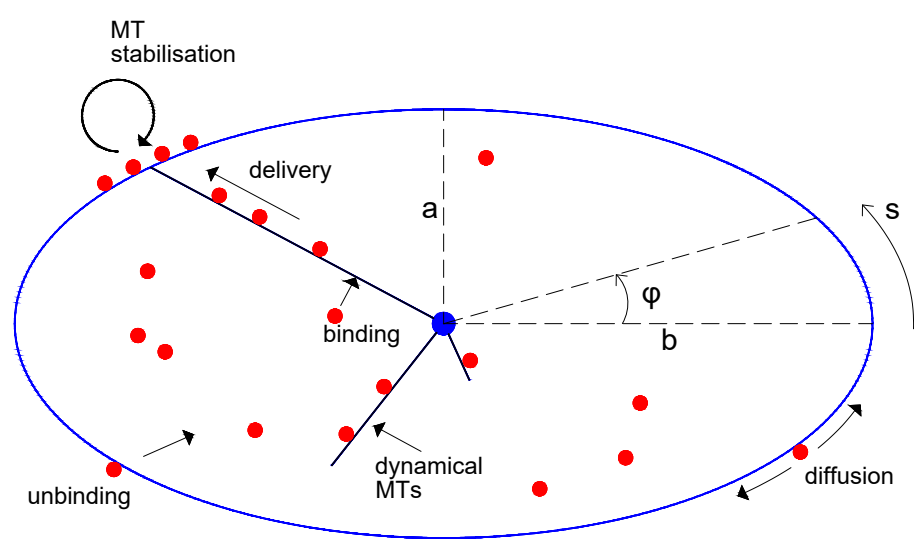

Figure 7.5: Schematic of the elliptical model without microtubule sliding. The ellipse length coordinate $s$ substitutes the angular coordinate $\phi$ of the spherical model, which now stands only for the nucleation angle.

In the simulations executed in the rest of Chapter 7 , we use the parameters shown in Table 7.1 for a time step $\Delta t=0.5 \mathrm{~s}$. We apply our algorithm with as initial condition all the microtubules and the polarity factors confined to a small region of one of the short axes endpoints of the ellipse.

Regarding the simulation results, for the value of $C=10000, S_{1}$ converges asymptotically to zero while the bipolar order parameter $S_{2}$ converges to negative values (Fig 7.6). This means, that the transverse bipolar phase is established, which is confirmed by the distribution shown in figure 7.7. The same happens for the higher value of $\mathrm{C}=40000$, the distribution of which is shown in figure 7.10 . 


\begin{tabular}{ll}
\hline Parameter & Simulation value \\
\hline $\mathrm{b}$ & $4 \mu \mathrm{m}$ \\
$\mathrm{a}$ & $2 \mu \mathrm{m}$ \\
$v_{+}$ & $0.018 \mu \mathrm{m} \mathrm{s}^{-1}$ \\
$v_{-}$ & $0.040 \mu \mathrm{m} \mathrm{s}^{-1}$ \\
$r_{n}$ & $0.05 \mathrm{~s}^{-1}$ \\
$r_{+}$ & $0.0078 \mathrm{~s}^{-1}$ \\
$r_{-}$ & $0.0016 \mathrm{~s}^{-1}$ \\
$\beta d$ & $0.60 \mathrm{pN}^{-1}$ \\
$k$ & $0.3{\mathrm{pN} \mu \mathrm{m}^{-1}}$ \\
$\mathrm{MTs}$ & 1000 \\
$\mathrm{M}$ & 400 \\
$k_{u}$ & $0.07 \mathrm{~s}^{-1}$ \\
$\mathrm{D}$ & $0.035 \mu \mathrm{m}^{2} \mathrm{~s}^{-1}$ \\
$(M / 3) c_{*}$ & $20 \times 10^{3}$ \\
$r_{u}(0)$ & $0.01 \mathrm{~s}^{-1}$ \\
$r_{u}(\infty)$ & $0.001 \mathrm{~s}^{-1}$ \\
$v_{m}$ & $0.81 \mu \mathrm{m} \mathrm{s}^{-1}$ \\
$l_{1 / 2}$ & $150 \mu \mathrm{m}^{\mathrm{p}}$ \\
\hline
\end{tabular}

Table 7.1: Model parameters used in the simulations of Chapter 7.

For $C=20000$, the unipolar order parameter $S_{1}$ converges asymptotically to a significant non-zero value, as we can see in figure 7.8. In that way, the establishment of a transverse unipolar phase is proved. The distribution profile coming out from the simulations (Fig 7.9$)$, also depicts this phase.

After executing a large number of simulations with different values of $C$, we are able to construct the graph of the unipolar order parameter $S_{1}$ as a function of $C$ and the corresponding graph of the bipolar order parameter $S_{2}$. The two graphs are shown in figure 7.11. The existence of three areas of $C$ values (two with a transverse bipolar ordering and one with a unipolar ordering), which we detected earlier by running simulations for $\mathrm{C}=10000$, $\mathrm{C}=20000$ and $\mathrm{C}=40000$, are now presented in this figure. The absence of the sliding effect of microtubules from our analysis, restricts the presence of the bipolar phase to the transverse direction of the ellipse. 

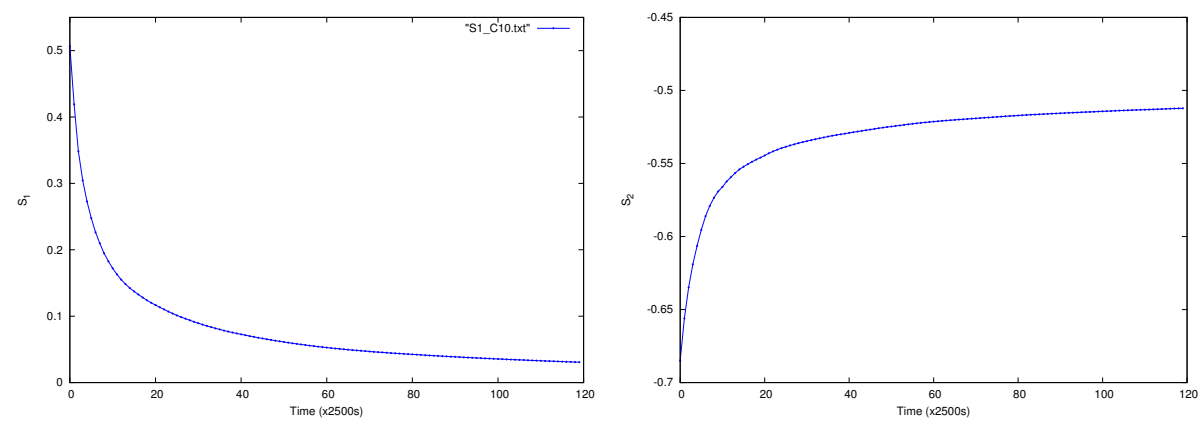

Figure 7.6: Order parameters $S_{1}, S_{2}$ as functions of time for $C=10000$. Initial condition: all the microtubules and the polarity factors are bound to a small region round $\phi=\pi / 2 . S_{1}$ converges asymptotically with time to zero while $S_{2}$ converges to a negative value, supporting a transverse bipolar phase.

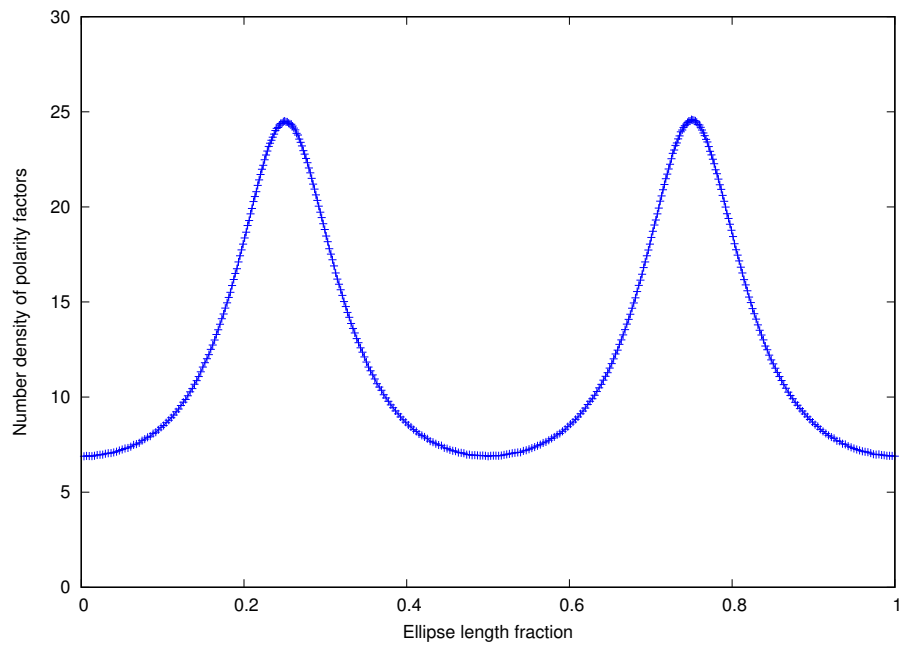

Figure 7.7: Transverse bipolar phase with no sliding for $C=10000$. 


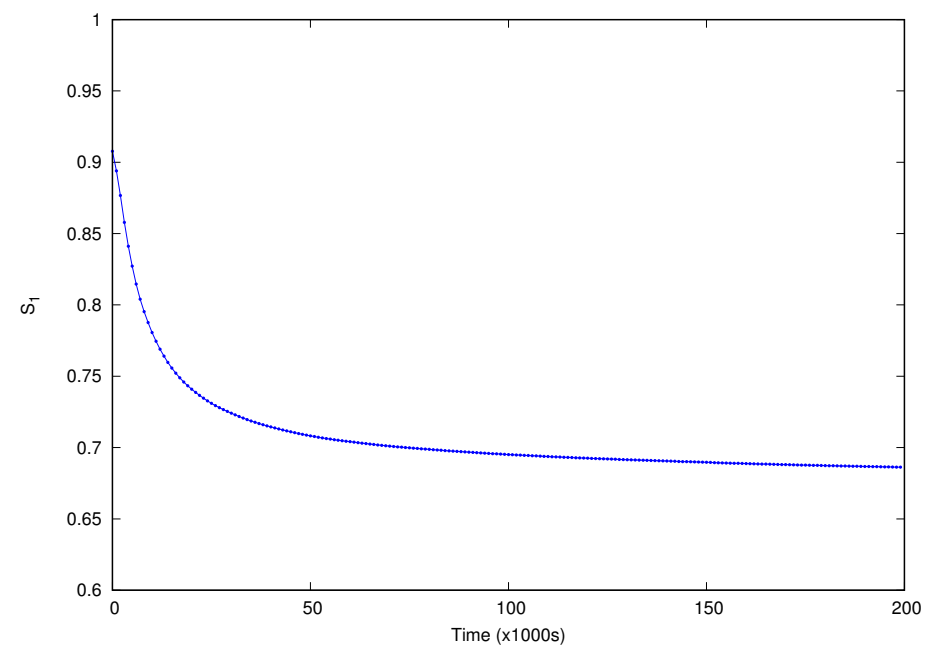

Figure 7.8: Order parameter $S_{1}$ as a function of time for $C=20000$. Initial condition: all the microtubules and the polarity factors are bound to a small region round $\phi=\pi / 2 . S_{1}$ converges asymptotically with time to a non-zero value, supporting a transverse unipolar phase.

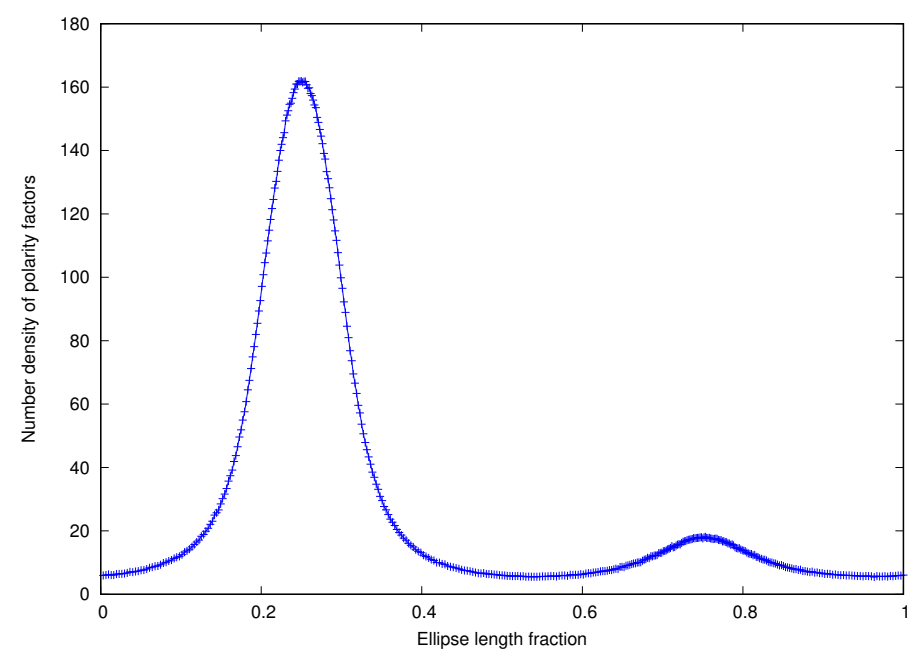

Figure 7.9: Transverse unipolar phase with no sliding for $C=20000$. 


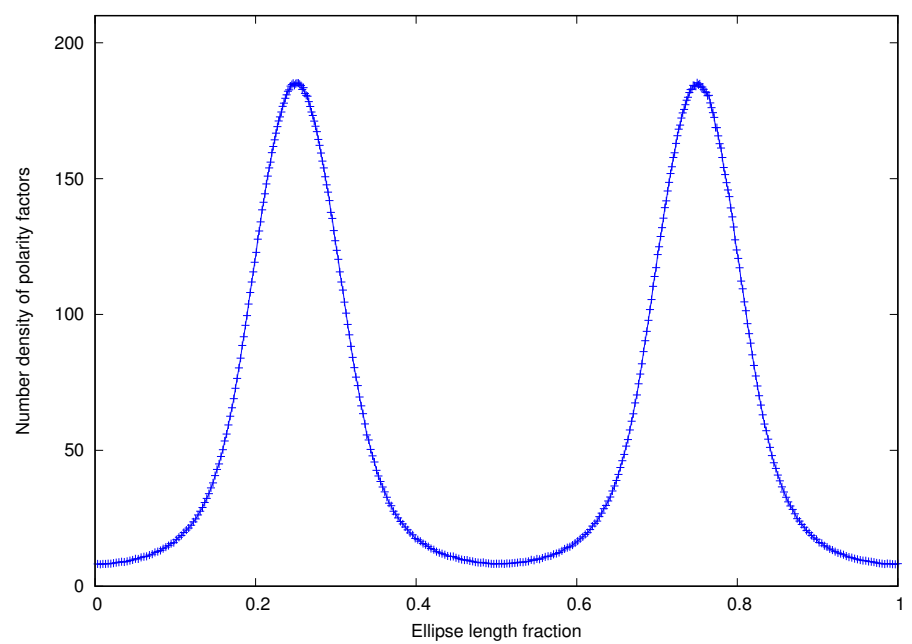

Figure 7.10: Transverse bipolar phase with no sliding for $C=40000$.

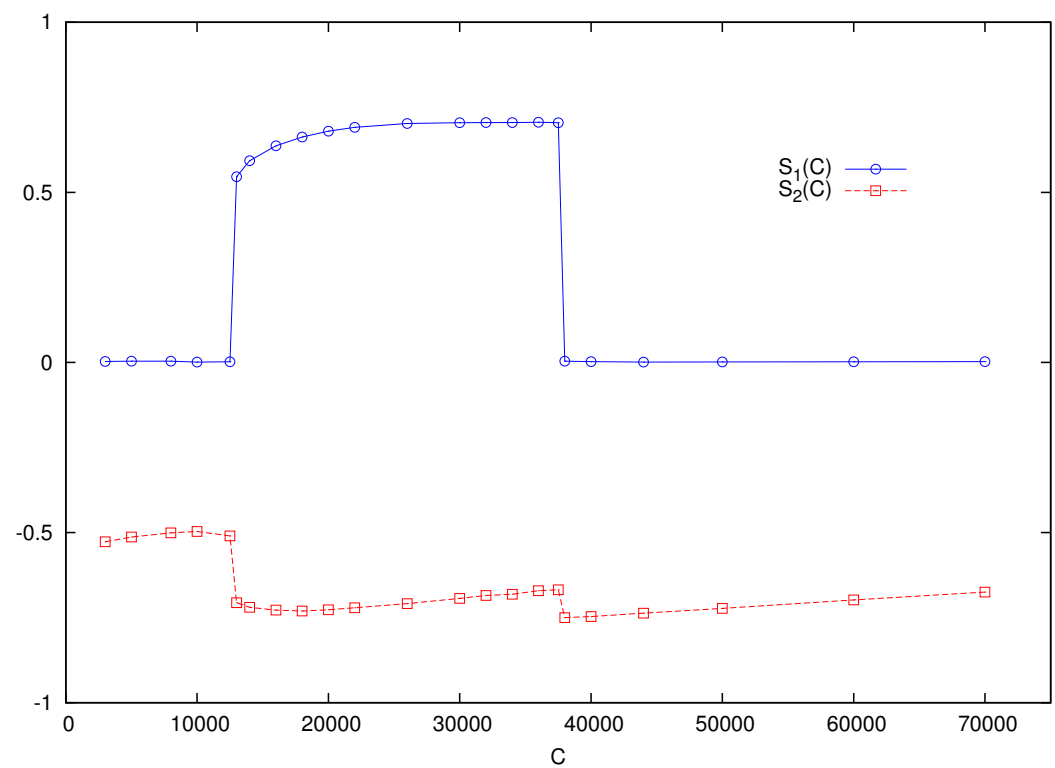

Figure 7.11: Transverse unipolar and bipolar order parameters $S_{1}$ and $S_{2}$ in the case of no sliding. The negative value of $S_{2}$ restricts the bipolar phase at the transverse direction. 


\subsubsection{Polarization in the presence of sliding}

Here we recall the simulation algorithm developed for the sliding of microtubules in Chapter 4. This algorithm is suitable as it concerns the states of the microtubules, but needs a modification in order to include the polarity mechanism. So, we modify it following the previously developed introduction of the polarity mechanism to the boundary-sliding model. The new model is presented in figure 7.12 .

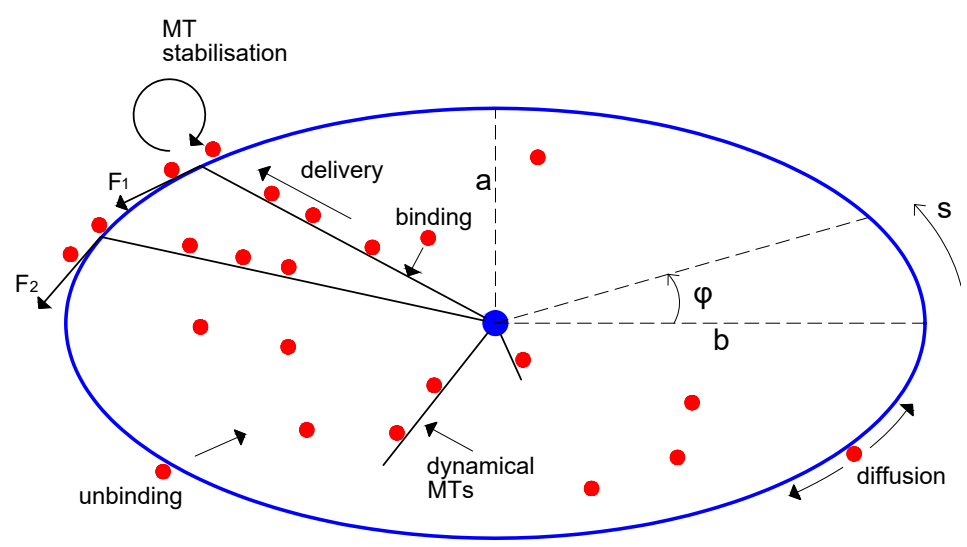

Figure 7.12: Schematic of the elliptical model including microtubule sliding. The microtubules slide on the boundary due to the tangential components (like $F_{1}, F_{2}$ ) of the pushing forces.

In the first simulations that we executed and for certain numbers of polarity factors $C$, a change of the polarization from transverse bipolar to unipolar showed up. This typically occurred after a large number of the system's time steps. This fact suggests the existence of a metastable state, which the polarity factors have to overcome during the evolution of the system. 
In order to circumvent a possible metastable state, we vary the initial conditions of our simulations. Intending to examine thoroughly the interplay between sliding, shape and the polarity mechanism, we regard three different initial condition-cases for the microtubules and the polarity factors. Each initial condition is chosen as to introduce the strongest bias that opposes a possible resultant state of the system. More specifically, we apply the transverse unipolar initial condition to the boundary position with $\phi=\pi / 2$, the longitudinal unipolar condition at the point with $\phi=0$ and the longitudinal bipolar condition. Additionally to these cases, we examine the case of the isotropic nucleation from the center of the ellipse.

Regarding the simulation results, figure 7.13 shows that for $\mathrm{C}=14000$, $S_{1}$ converges asymptotically to zero and $S_{2}$ converges to a negative value. So, a stable transverse bipolar phase is established. This is confirmed also by the simulation distribution shown in figure 7.14, which corresponds to the isotropic nucleation case.

Figure 7.15 shows the stability of the longitudinal unipolar phase established for $\mathrm{C}=22000$. Figures 7.16 and $(7.17)$ present unipolar distributions developed from the isotropic nucleation at different endpoints of the long semi-axes of the ellipse, which implies that the site of unipolar ordering on the longitudinal direction is chosen randomly among the two possible boundary sites.
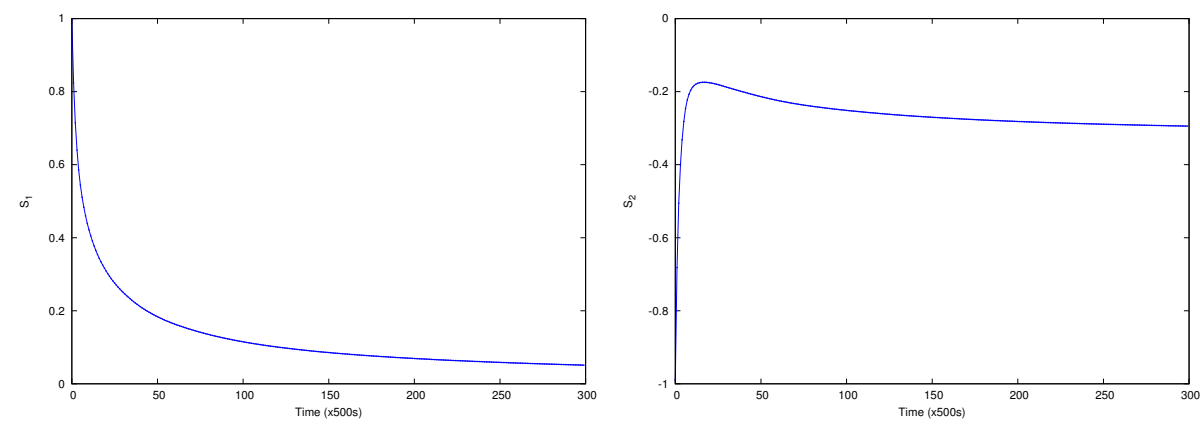

Figure 7.13: Order parameters $S_{1}, S_{2}$ calculated for each time value from the mean of the history distributions for the case $C=14000$. The unipolar phase is excluded as $S_{1}$ converges to zero and the transverse bipolar phase dominates as $S_{2}$ converges to a negative value. 


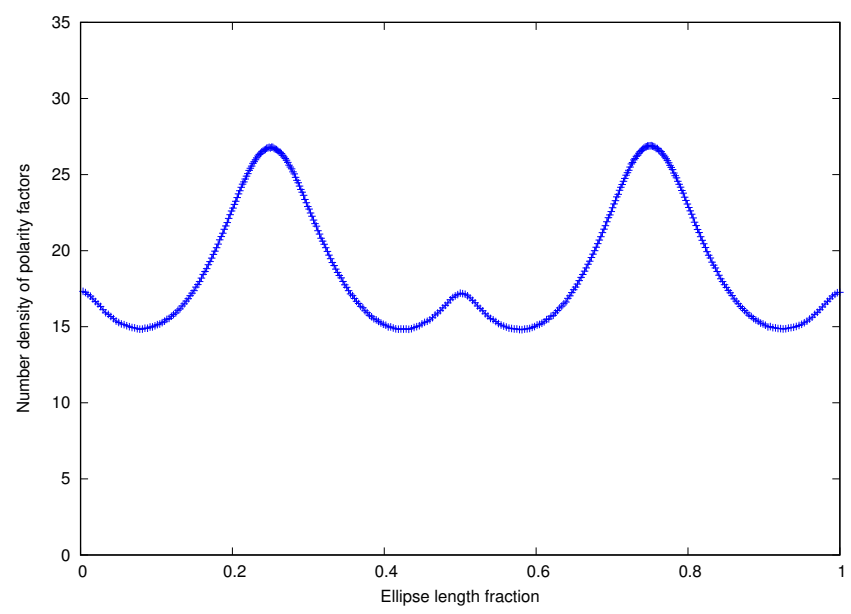

Figure 7.14: Number density of the polarity factors on the boundary for the case of isotropic nucleation with $C=14000$. The transverse bipolar phase is established.

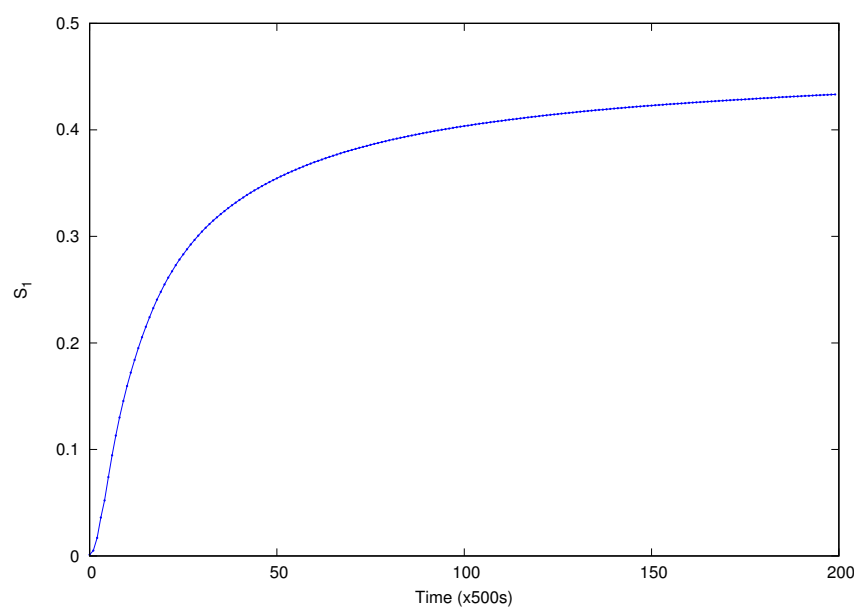

Figure 7.15: Order parameter $S_{1}$ calculated for each time value from the mean of the history distributions for the case $C=22000$. The unipolar phase dominates as $S_{1}$ converges to a non-zero value. There is no specific unipolar or bipolar initial condition, but the isotropic nucleation is applied. 


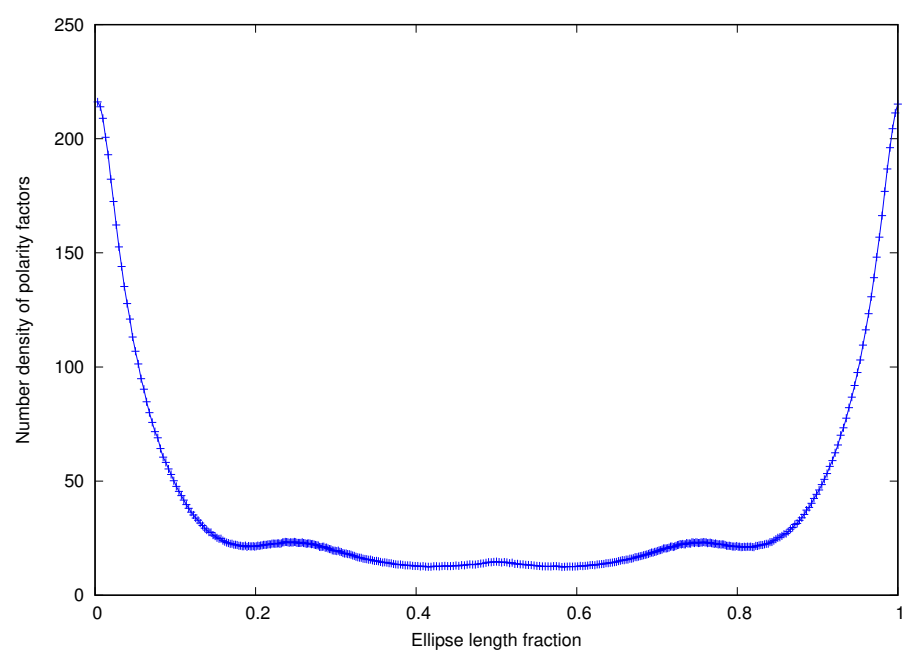

Figure 7.16: Number density of the polarity factors on the boundary for the case of the isotropic nucleation with $C=22000$. The longitudinal unipolar phase can appear at the boundary site with $\phi=0$.

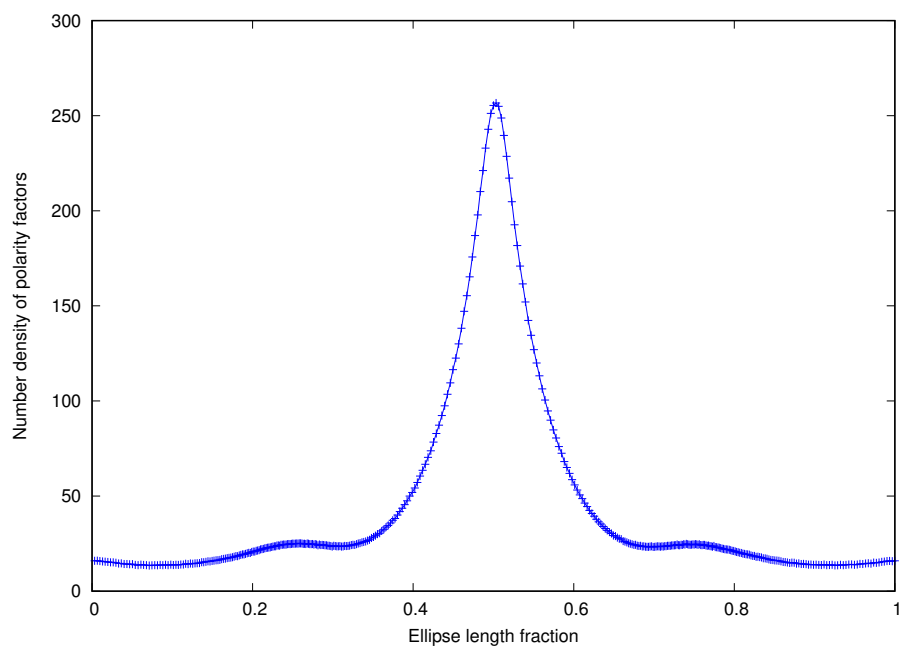

Figure 7.17: Number density of the polarity factors on the boundary for the case of the isotropic nucleation with $C=25000$. The longitudinal unipolar phase can appear at the boundary site with $\phi=\pi$. 
Figure 7.18 shows that for $\mathrm{C}=45000, S_{1}$ converges asymptotically to zero and $S_{2}$ converges to a positive value. So, a stable longitudinal bipolar phase is established. This is confirmed by the simulation distribution shown in figure 7.19, which comes out from the isotropic nucleation.
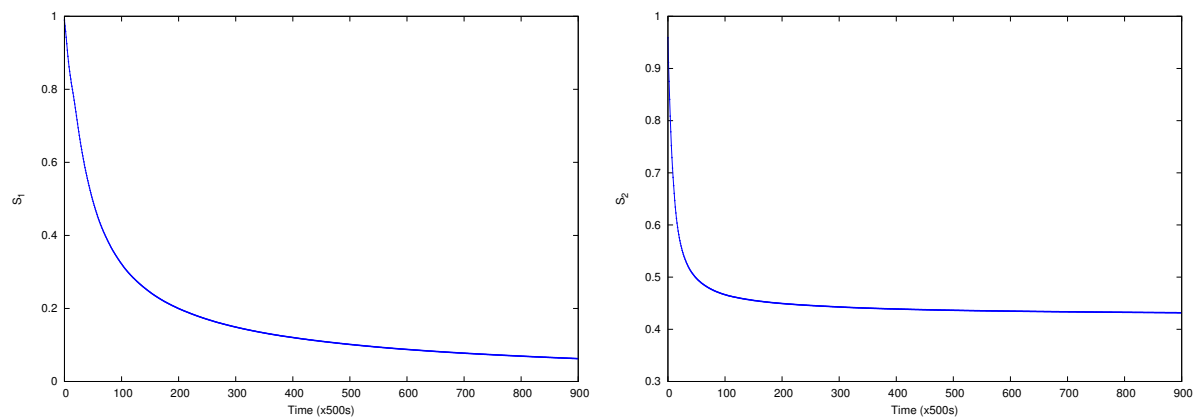

Figure 7.18: Order parameters $S_{1}, S_{2}$ calculated for each time value from the mean of the history distributions for the case $C=45000$. The unipolar phase is excluded as $S_{1}$ converges to zero and the longitudinal bipolar phase dominates as $S_{2}$ converges to a positive value.

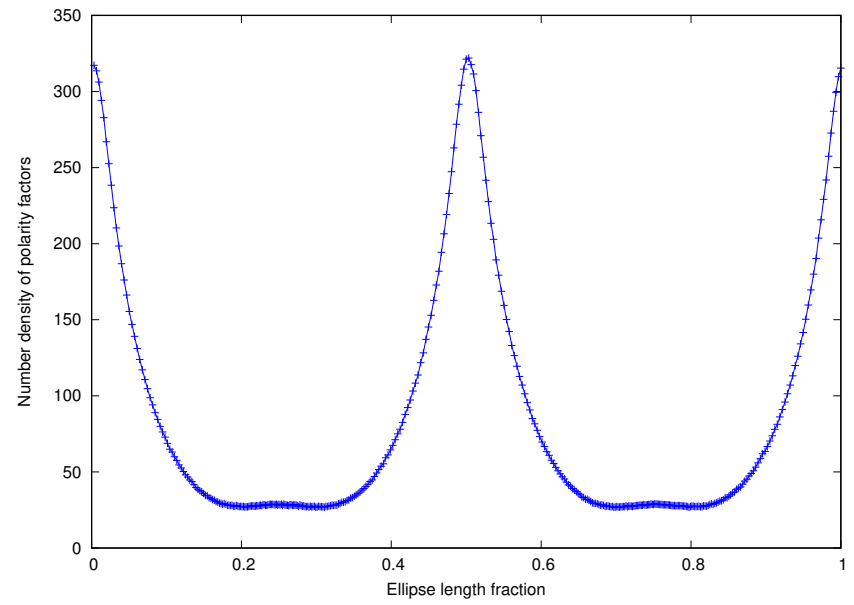

Figure 7.19: Number density of the polarity factors on the boundary for the case of the isotropic nucleation with $C=45000$. The longitudinal bipolar phase dominates. 
Executing a large number of simulations with different values of $C$ and for all four simulation cases, we construct the graphs of the unipolar order parameter $S_{1}$ and the bipolar order parameter $S_{2}$ as functions of time. The two graphs are shown in figures 7.20 and 7.21 where the existence of three areas of $C$ values with longitudinal bipolar or unipolar ordering are presented.

An important conclusion about the function of the dynamical system examined comes from the observation of $S_{2}(t)$ graph. In figure 7.21 we can see that the system shows the phenomenon of hysteresis. Indeed, there is a regime of $C$ values, which can support two alternative stable states. For the initial condition with $\phi=0$, the system supports the longitudinal unipolar state, while for the other initial conditions examined and the isotropic nucleation case supports the longitudinal bipolar state.

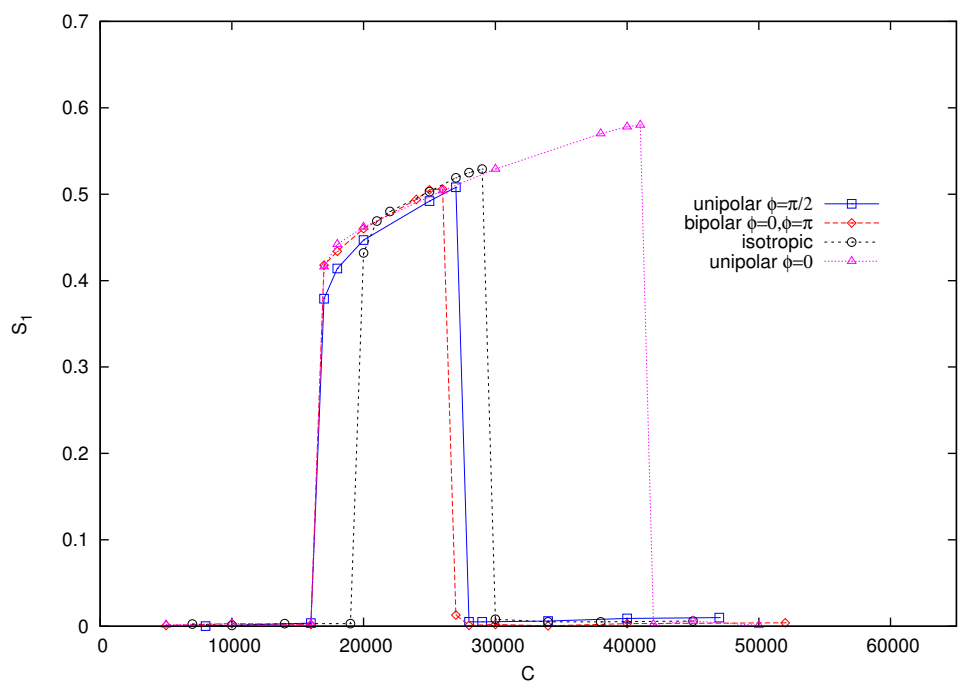

Figure 7.20: Unipolar order parameters $S_{1}$ in the case of sliding. The simulation results shown correspond to the cases of three initial conditions for the microtubules and the polarity factors plus the case of the isotropic nucleation. The extended unipolar phase of the case $\phi=0$ in relation with the other cases reveals a hysteresis effect. 


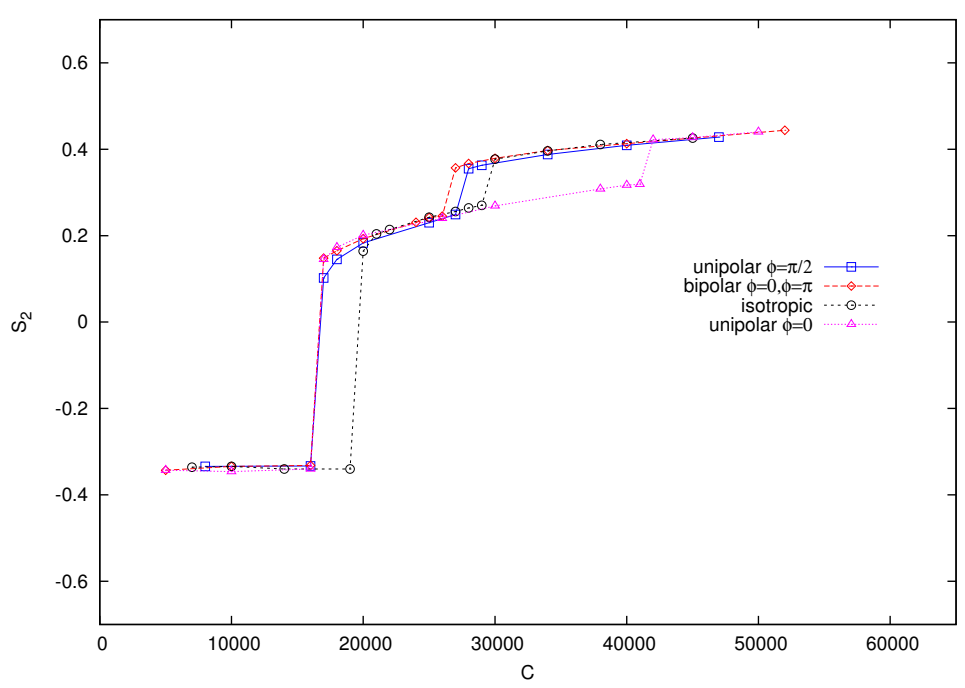

Figure 7.21: Bipolar order parameter $S_{2}$ in the case of sliding. We can see the hysteresis loop distinguishing the initial condition at $\phi=0$ from the other three cases.

\subsubsection{Analytical model of perfect bipolar biasing}

The unipolar ordering, even for a certain regime of $C$ values, is a result we initially did not expect in the presence of sliding. In order to prove analytically that indeed the unipolar state is inevitable in the system, we consider a simple model with the strongest possible bipolar bias. Regarding an ellipse with the endpoints of the long semi-axes at angles $\theta=0$ and $\theta=$ $\pi$, we want to maximally bias the microtubules towards these points. So, all the microtubules (nucleated from the center of the ellipse) are oriented from the outset at angles $\theta=0$ and $\theta=\pi$, and the corresponding poles are the only delivery points for the polarity factors.

Then, the diffusion equation for the polarity factors in the boundary is of the form

$$
D \frac{\partial^{2}}{\partial \vartheta^{2}} c_{b}(\varphi)-k_{u} c_{b}(\vartheta)+K_{b}\left(c_{b}(0)\right) \delta(\vartheta)+K_{b}\left(c_{b}(\pi)\right) \delta(\vartheta-\pi)=0
$$

with $K_{b}$ set as

$$
K_{b}\left(c_{b}(\theta)\right)=u_{m} c_{m}\left(c_{b}(\theta)\right) m_{b}\left(c_{b}(\theta)\right)
$$


We know from the spherical polarity problem, that the number of the bound microtubules is given by the relation

$$
m_{b}(\theta)=\frac{m \mu_{b} r_{n}}{\left(1+\mu_{i}\right) r_{u}\left(c_{b}(\theta)\right)+\mu_{b} r_{n}}
$$

which in our case becomes

$$
m_{b}(\theta)=m_{b}(\theta=0)+m_{b}(\theta=\pi)
$$

We set $\Delta r_{u}=r_{u}(0)-r_{u}(\infty)$. Then, we can write

$$
r_{u}\left(c_{b}(\theta)\right)=\Delta r_{u} \sigma\left(c_{b}(\theta) / c_{*}\right)+r_{u}(\infty)
$$

and following

$$
m_{b}\left(c_{b}(\theta)\right)=\frac{m \mu_{b} r_{n}}{\left(1+\mu_{i}\right)\left(\Delta r_{u} \sigma\left(c_{b}(\theta) / c_{*}\right)+r_{u}(\infty)\right)+\mu_{b} r_{n}}
$$

We also know that the density $c_{m}$ of polarity factors that are bound on the microtubules is given by the relation

$$
c_{m}\left(c_{b}(\theta)\right)=\frac{C-\int_{0}^{2 \pi} d \theta c_{b}(\theta)}{l_{\text {tot }}\left(c_{b}(\theta)\right)+l_{1 / 2}}
$$

We set

$\Lambda\left(c_{b}(\theta)\right)=l_{\text {tot }}\left(c_{b}(\theta)\right)+l_{1 / 2}=m \bar{l} \int_{0}^{2 \pi} d \theta \frac{\lambda_{i} r_{u}\left(c_{b}(\theta)\right)+\lambda_{b} r_{n}}{\left(1+\mu_{i}\right) r_{u}\left(c_{b}(\theta)\right)+\mu_{b} r_{n}}+l_{1 / 2}$

We first consider the bipolar reference solution, for which $c_{b}^{(0)}(\vartheta)=$ $c_{b}^{(0)}(\pi-\vartheta)$, so that $K_{b}\left(c_{b}^{(0)}(0)\right)=K_{b}\left(c_{b}^{(0)}(\pi)\right)$. The reference solution is the solution with the maximum symmetry. We also recall that

$$
\delta(\vartheta)=\frac{1}{2 \pi}+\frac{1}{\pi} \sum_{n=1}^{\infty} \cos (n \vartheta) .
$$

The reference solution thus satisfies

$$
D \frac{\partial^{2}}{\partial \vartheta^{2}} c_{b}^{(0)}(\vartheta)-k_{u} c_{b}^{(0)}(\vartheta)+K_{b}\left(c_{b}^{(0)}(0)\right)\{\delta(\vartheta)+\delta(\vartheta-\pi)\}=0
$$


The solution to this equation is

$$
c_{b}^{(0)}(\vartheta)=K_{b}\left(c_{b}^{(0)}(0)\right)\left\{\frac{1}{2 \pi k_{u}}+\frac{1}{\pi} \sum_{n=1}^{\infty} \frac{1}{\left(4 D n^{2}+k_{u}\right)} \cos (2 n \vartheta)\right\}
$$

Self-consistency requires that

$$
\begin{aligned}
c_{b}^{(0)}(0) & =K_{b}\left(c_{b}^{(0)}(0)\right)\left\{\frac{1}{2 \pi k_{u}}+\frac{1}{\pi} \sum_{n=1}^{\infty} \frac{1}{\left(4 D n^{2}+k_{u}\right)}\right\} \\
& =K_{b}\left(c_{b}^{(0)}(0)\right) \frac{\operatorname{coth}\left(\frac{\pi}{2} \sqrt{\frac{k_{u}}{D}}\right)}{4 \sqrt{k_{u} D}} .
\end{aligned}
$$

This is an implicit, non-linear relation that fixes $c_{b}^{(0)}(0)$ for every $C$. We consider now the general solution to 7.27

$$
c_{b}(\vartheta)=\sum_{n=0}^{\infty} c_{n} \cos (n \vartheta)
$$

We find

$$
\begin{array}{r}
-k_{u} c_{0}+\sum_{n=1}^{\infty}\left(-n^{2} D-k_{u}\right) c_{n} \cos (n \vartheta)+\frac{1}{2 \pi}\left\{K_{b}\left(c_{b}(0)\right)+K_{b}\left(c_{b}(\pi)\right)\right\} \\
+\frac{1}{\pi} \sum_{n=1}^{\infty}\left\{K_{b}\left(c_{b}(0)\right)+(-)^{n} K_{b}\left(c_{b}(\pi)\right)\right\} \cos (n \vartheta)=0
\end{array}
$$

So

$$
\begin{aligned}
c_{0} & =\frac{1}{2 \pi k_{u}}\left\{K_{b}\left(c_{b}(0)\right)+K_{b}\left(c_{b}(\pi)\right)\right\} \\
c_{2 n-1} & =\frac{1}{\pi}\left\{\frac{K_{b}\left(c_{b}(0)\right)-K_{b}\left(c_{b}(\pi)\right)}{\left((2 n-1)^{2} D+k_{u}\right)}\right\}, n \geq 1 \\
c_{2 n} & =\frac{1}{\pi}\left\{\frac{K_{b}\left(c_{b}(0)\right)+K_{b}\left(c_{b}(\pi)\right)}{\left((2 n)^{2} D+k_{u}\right)}\right\}, n \geq 1
\end{aligned}
$$


Thus

$$
\begin{aligned}
c_{b}(\vartheta) & =\frac{1}{2 \pi k_{u}}\left\{K_{b}\left(c_{b}(0)\right)+K_{b}\left(c_{b}(\pi)\right)\right\} \\
& +\frac{1}{\pi} \sum_{n=1}^{\infty}\left\{\frac{K_{b}\left(c_{b}(0)\right)+K_{b}\left(c_{b}(\pi)\right)}{\left((2 n)^{2} D+k_{u}\right)}\right\} \cos (2 n \vartheta) \\
& +\frac{1}{\pi} \sum_{n=1}^{\infty}\left\{\frac{K_{b}\left(c_{b}(0)\right)-K_{b}\left(c_{b}(\pi)\right)}{\left((2 n-1)^{2} D+k_{u}\right)}\right\} \cos ((2 n-1) \vartheta)
\end{aligned}
$$

The self-consistency equations are

$$
\begin{aligned}
c_{b}(0) & =\left\{K_{b}\left(c_{b}(0)\right)+K_{b}\left(c_{b}(\pi)\right)\right\} \frac{\operatorname{coth}\left(\frac{\pi}{2} \sqrt{\frac{k_{u}}{D}}\right)}{4 \sqrt{k_{u} D}} \\
& +\left\{K_{b}\left(c_{b}(0)\right)-K_{b}\left(c_{b}(\pi)\right)\right\} \frac{\tanh \left(\frac{\pi}{2} \sqrt{\frac{k_{u}}{D}}\right)}{4 \sqrt{k_{u} D}} \\
c_{b}(\pi) & =\left\{K_{b}\left(c_{b}(0)\right)+K_{b}\left(c_{b}(\pi)\right)\right\} \frac{\operatorname{coth}\left(\frac{\pi}{2} \sqrt{\frac{k_{u}}{D}}\right)}{4 \sqrt{k_{u} D}} \\
& -\left\{K_{b}\left(c_{b}(0)\right)-K_{b}\left(c_{b}(\pi)\right)\right\} \frac{\tanh \left(\frac{\pi}{2} \sqrt{\frac{k_{u}}{D}}\right)}{4 \sqrt{k_{u} D}}
\end{aligned}
$$

It is useful to introduce the shorthands

$$
\begin{array}{r}
B=\frac{\operatorname{coth}\left(\frac{\pi}{2} \sqrt{\frac{k_{u}}{D}}\right)}{4 \sqrt{k_{u} D}} \\
P=\frac{\tanh \left(\frac{\pi}{2} \sqrt{\frac{k_{u}}{D}}\right)}{4 \sqrt{k_{u} D}}
\end{array}
$$

It is clear that the bipolar solution corresponds to the choice $c_{b}(0)=$ $c_{b}(\pi)=\overline{c_{b}}$, in which case it reduces to

$$
\overline{c_{b}}=2 B K_{b}\left(\overline{c_{b}}\right)
$$


We now attempt to linearize around this solution setting

$$
\begin{aligned}
c_{b}(0) & =\bar{c}_{b}+\varepsilon_{0} \\
c_{b}(\pi) & =\bar{c}_{b}-\varepsilon_{\pi}
\end{aligned}
$$

Initially, we calculate the general perturbation $\epsilon$ to $m_{b}$, which is either $m_{b}(\phi=0)$ or $m_{b}(\phi=\pi)$. Later, in the place of $\epsilon$, we'll put either $\epsilon_{0}$ or $\epsilon_{\pi}$. We use the relation

$$
r_{u}\left(\bar{c}_{b}+\epsilon\right)=r_{u}\left(\bar{c}_{b}\right)+\Delta r_{u} \frac{\epsilon}{c_{*}} \sigma^{\prime}\left(\bar{c}_{b} / c_{*}\right)
$$

and we get

$$
\begin{aligned}
& m_{b}\left(\bar{c}_{b}+\epsilon\right)=\frac{(m / 2) \mu_{b} r_{n}}{\left(1+\mu_{i}\right)\left(\Delta r_{u} \sigma\left(\bar{c}_{b} / c_{*}\right)+r_{u}(\infty)+\Delta r_{u}\left(\epsilon / c_{*}\right) \sigma^{\prime}\left(\bar{c}_{b} / c_{*}\right)\right)+\mu_{b} r_{n}} \\
& =\frac{(m / 2) \mu_{b} r_{n}}{\left(\left(1+\mu_{i}\right)\left(\Delta r_{u} \sigma\left(\bar{c}_{b} / c_{*}\right)+r_{u}(\infty)\right)+\mu_{b} r_{n}\right)\left(1+\frac{\left(1+\mu_{i}\right) \Delta r_{u}\left(\epsilon / c_{*}\right) \sigma^{\prime}\left(\bar{c}_{b} / c_{*}\right)}{\left(1+\mu_{i}\right)\left(\Delta r_{u} \sigma\left(\bar{c}_{b} / c_{*}\right)+r_{u}(\infty)\right)+\mu_{b} r_{n}}\right)} \\
& =m_{b}\left(\bar{c}_{b}\right) \frac{1}{\left(1+\frac{\Delta r_{u}\left(\epsilon / c_{*}\right) \sigma^{\prime}\left(\bar{c}_{b} / c_{*}\right)}{\left.\left(\Delta r_{u} \sigma\left(\bar{c}_{b} / c_{*}\right)+r_{u}(\infty)\right)+\frac{\mu_{b} r_{n}}{1+\mu_{i}}\right)}\right)} \\
& =m_{b}\left(\bar{c}_{b}\right) \frac{1}{\left(1+\frac{\frac{\Delta r_{u}}{r_{u}(0)}\left(\epsilon / c_{*}\right) \sigma^{\prime}\left(\bar{c}_{b} / c_{*}\right)}{\left.\left(\frac{\Delta r_{u}}{r_{u}(0)} \sigma\left(\bar{c}_{b} / c_{*}\right)+\frac{r_{u}(\infty)}{r_{u}(0)}\right)+\frac{\mu_{b} r_{n}}{\left(1+\mu_{i}\right) r_{u}(0)}\right)}\right.} \\
& =m_{b}\left(\bar{c}_{b}\right) \frac{1}{\left(1+\frac{\left(\epsilon / c_{*}\right) \sigma^{\prime}\left(\bar{c}_{b} / c_{*}\right)}{\sigma\left(\bar{c}_{b} / c_{*}\right)+\eta}\right)} \\
& =m_{b}\left(\bar{c}_{b}\right)\left(1-\frac{\left(\epsilon / c_{*}\right) \sigma^{\prime}\left(\bar{c}_{b} / c_{*}\right)}{\sigma\left(\bar{c}_{b} / c_{*}\right)+\eta}\right) \\
& =m_{b}\left(\bar{c}_{b}\right)-m_{b}\left(\bar{c}_{b}\right) \frac{\sigma^{\prime}\left(\bar{c}_{b} / c_{*}\right)}{\sigma\left(\bar{c}_{b} / c_{*}\right)+\eta} \frac{\epsilon}{c_{*}}
\end{aligned}
$$

where $\eta$ is the known quantity from the spherical polarity problem

$$
\eta=\left(\frac{r_{u}(\infty)}{r_{u}(0)}+\frac{m_{b} r_{n}}{\left(1+\mu_{i}\right) r_{u}(0)}\right) /\left(1-\frac{r_{u}(\infty)}{r_{u}(0)}\right)
$$

Now, we apply the perturbation to $c_{m}$. First, we apply it to the number of the free polarity factors

$$
C-\int_{0}^{2 \pi} d \theta\left(\bar{c}_{b}(0)+\epsilon_{0}\right) \delta(\theta)-\int_{0}^{2 \pi} d \theta\left(\bar{c}_{b}(\pi)-\epsilon_{\pi}\right) \delta(\theta-\pi)=C-2 \bar{c}_{b}
$$


and, then, as a general perturbation $\epsilon$ to $\bar{c}_{b}$ in $\Lambda\left(\bar{c}_{b}\right)$

$$
\Lambda\left(\bar{c}_{b}+\epsilon\right)=m \bar{l} \int_{0}^{2 \pi} d \theta \frac{\lambda_{i}\left(r_{u}\left(\bar{c}_{b}\right)+\Delta r_{u}\left(\epsilon / c_{*}\right) \sigma^{\prime}\left(\bar{c}_{b} / c_{*}\right)\right)+\lambda_{b} r_{n}}{\left(1+\mu_{i}\right)\left(r_{u}\left(\bar{c}_{b}\right)+\Delta r_{u}\left(\epsilon / c_{*}\right) \sigma^{\prime}\left(\bar{c}_{b} / c_{*}\right)\right)+\mu_{b} r_{n}}+l_{1 / 2}
$$

We approximate the integral by neglecting the contribution of the term multiplied by $\epsilon$

$$
\Lambda\left(\bar{c}_{b}+\epsilon\right)=2 \pi m \bar{l} \frac{\lambda_{i} r_{u}\left(\bar{c}_{b}\right)+\lambda_{b} r_{n}}{\left(1+\mu_{i}\right) r_{u}\left(\bar{c}_{b}\right)+\mu_{b} r_{n}}+l_{1 / 2}=\Lambda\left(\bar{c}_{b}\right)
$$

So, it holds

$$
c_{m}\left(\bar{c}_{b}+\epsilon\right)=c_{m}\left(\bar{c}_{b}\right)
$$

Now, we can write

$$
\begin{aligned}
K_{b}\left(\bar{c}_{b}+\epsilon\right) & =v_{m} c_{m}\left(\bar{c}_{b}\right) m_{b}\left(\bar{c}_{b}+\epsilon\right) \\
& =v_{m} c_{m}\left(\bar{c}_{b}\right)\left(m_{b}\left(\bar{c}_{b}\right)-m_{b}\left(\bar{c}_{b}\right) \frac{\sigma^{\prime}\left(\bar{c}_{b} / c_{*}\right)}{\sigma\left(\bar{c}_{b} / c_{*}\right)+\eta} \frac{\epsilon}{c_{*}}\right) \\
& =K_{b}\left(\bar{c}_{b}\right)-K_{b}\left(\bar{c}_{b}\right) \frac{\sigma^{\prime}\left(\bar{c}_{b} / c_{*}\right)}{\sigma\left(\bar{c}_{b} / c_{*}\right)+\eta} \frac{\epsilon}{c_{*}}=K_{b}\left(\bar{c}_{b}\right)+K_{b}^{\prime}\left(\bar{c}_{b}\right) \epsilon
\end{aligned}
$$

with

$$
K_{b}^{\prime}\left(\bar{c}_{b}\right)=-K_{b}\left(\bar{c}_{b}\right) \frac{1}{c_{*}} \frac{\sigma^{\prime}\left(\bar{c}_{b} / c_{*}\right)}{\sigma\left(\bar{c}_{b} / c_{*}\right)+\eta}
$$

We substitute the perturbed values $K_{b}\left(\bar{c}_{b}+\epsilon_{0}\right)$ and $K_{b}\left(\bar{c}_{b}-\epsilon_{\pi}\right)$ to the self-consistency equations (7.44) and (7.45), which leads to

$$
\begin{aligned}
& \varepsilon_{0}=B K_{b}^{\prime}\left(\overline{c_{b}}\right)\left(\varepsilon_{0}-\varepsilon_{\pi}\right)+P K_{b}^{\prime}\left(\overline{c_{b}}\right)\left(\varepsilon_{0}+\varepsilon_{\pi}\right) \\
& \varepsilon_{\pi}=B K_{b}^{\prime}\left(\overline{c_{b}}\right)\left(\varepsilon_{0}-\varepsilon_{\pi}\right)-P K_{b}^{\prime}\left(\overline{c_{b}}\right)\left(\varepsilon_{0}+\varepsilon_{\pi}\right)
\end{aligned}
$$

or

$$
\left(\begin{array}{cc}
1+K_{b}^{\prime}\left(\overline{c_{b}}\right)(B+P) & K_{b}^{\prime}\left(\overline{c_{b}}\right)(-B+P) \\
-K_{b}^{\prime}\left(\overline{c_{b}}\right)(-B+P) & 1-K_{b}^{\prime}\left(\overline{c_{b}}\right)(B+P)
\end{array}\right)\left(\begin{array}{c}
\varepsilon_{0} \\
\varepsilon_{\pi}
\end{array}\right)=\left(\begin{array}{c}
0 \\
0
\end{array}\right)
$$


which has a non negative solutions only if

$$
\left(1+K_{b}^{\prime}\left(\overline{c_{b}}\right)(B+P)\right)\left(1-K_{b}^{\prime}\left(\overline{c_{b}}\right)(B+P)\right)+\left(K_{b}^{\prime}\left(\overline{c_{b}}\right)(-B+P)\right)^{2}=0
$$

leading to the bifurcation condition

$$
K_{b}^{\prime}\left(\overline{c_{b}}\right)=\frac{1}{2 \sqrt{B P}}=2 \sqrt{k_{u} D}=2 k_{u} \sqrt{\delta}
$$

The bifurcation in our system, leads to the breaking of the bipolar symmetry and, so, to the establishment of the unipolar phase.

We can also see why one could not assume from the outset that the bifurcating solution is a pure unipolar perturbation. Indeed the assumption $c_{b}^{(1)}(\vartheta)=-c_{b}^{(1)}(\pi-\vartheta)$ would be incorrect. This is evident from 7.62 7.63), because setting $\varepsilon_{0}=\varepsilon_{\pi}$ yields two equations which are consistent only for $K_{b}^{\prime}\left(\overline{c_{b}}\right)=0$, i.e. when the bifurcation equation identically yields zero, i.e. where there is no perturbation.

\subsection{Conclusion}

In this chapter we focused on the polarization of an elliptical cell. We applied stochastic simulations in order to investigate the interaction between the shape, the sliding effect and the polarity mechanism. We considered both the without sliding and with microtubule sliding. The main conclusion in both cases is that the shape cannot impose its symmetry on system in every regime of the total number of polarity factors.

More specifically, in the case without sliding a win of the shape would be expressed by the presence of a transverse bipolar state for every value of the number of polarity factors, due to the intrinsic dynamics of the microtubules. But this is not happening, as the transverse unipolar phase dominates in a certain range of $C$ values. This is a win of the polarization mechanism against the shape.

In the presence of the sliding effect, a win of the shape would be the establishment of the longitudinal bipolar phase for every value of $C$. This is because the endpoints of the long semi-axes are equally favored by the sliding mechanism. However, the simulation results reveal the domination of the longitudinal unipolar state, again in a certain regime of $C$ values. This is a win of the polarization mechanism over the shape and the sliding effect. 
Summarizing, we can say that the polarization mechanism is able to override the effects both of the shape and the sliding mechanism and hence provides control over the polarity effect in elliptical cell. Except of the simulation results, this is confirmed also theoretically by the nucleation model with the strongest bipolar bias, which was also analyzed in this chapter. 


\section{Part IV}

\section{General discussion}





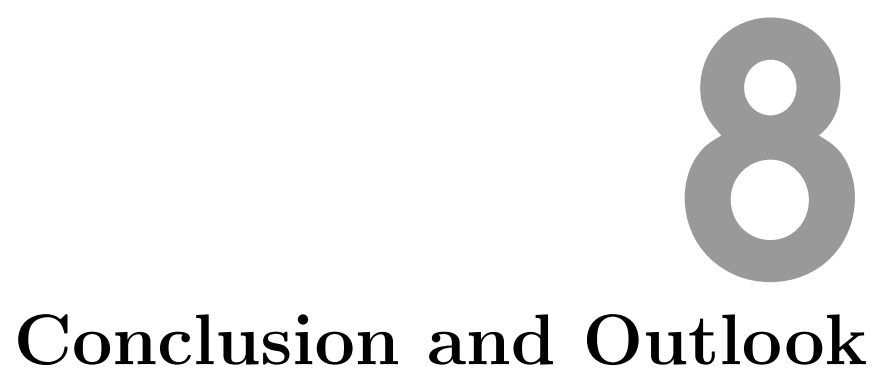

In this chapter we summarize the results from the earlier chapters and discuss them in a biological context. Following the present study, we suggest some possible new directions for future work. 


\subsection{Branched versus background nucleation}

In Chapter 2 we focused on the microtubules of the plant cortical array. We incorporated the phenomenon of microtubule-bound anisotropic nucleation to a theoretical framework previously developed. The co-alignment of the branched nucleations with the parent microtubules, was confirmed by a bifurcation analysis as the main determinant of the transition location to order. Proceeding with numerical analysis, we showed the robustness of our mean-field theory approach by comparing with particle-based simulation results. As a key conclusion, our analytical model confirmed the strong self-consistent positive feedback on microtubule ordering.

Apart from confirming earlier simulation results, our theoretical analysis reveals the existence of a single dimensionless parameter called $\beta$ that determines the relative influence of the branched and the background isotropic nucleation. The new parameter that we introduced, depends on the affinity of nucleation complexes for microtubules and the relative strength of their nucleation rates in the bound and the unbound state.

A numerical analysis shows that by increasing the parameter $\beta$ - reinforcing branched vs background nucleation —, we get a wider regime of the microtubule dynamical parameters for which the system is ordered. This means that the ordered state becomes more robust as it can resist higher variation of the system parameters, which is potentially biologically significant.

It would be interesting to extend the presented model in order to include a number of effects that are involved in the in vivo ordering process. Specifically, we mention the effects of minus-end treadmilling, microtubule severing and the finiteness of the available tubulin pool.

\subsection{Microtubule organization induced by shape}

\subsubsection{Stalling at the cell boundary}

In Chapter 3 we developed a 3D-model for centrosomal microtubules that can stall at the cell boundary and we investigated their spatial distribution. To this end, we derived the equations that describe the length distribution of microtubules for a general form of a boundary. We also defined 3D and $2 \mathrm{D}$ length order parameters and calculated how ordering is influenced when 
we change gradually the shape from a prolate to an oblate ellipsoid. Next, we showed that in the presence of an axial symmetry the behaviour of our model is not very sensitive to a change of dimension. This observation enabled us to apply 2D particle-based simulations, which are simpler and faster than the $3 \mathrm{D}$ ones.

A numerical analysis was applied to the case of an ellipse with a centrosome fixed at its center. The results revealed the existence of two directions of ordering. One was longitudinal and the other was transverse, each one dominating at a different regime of the system parameters. More specifically, high unbinding rates from the cell boundary and shorter short semi-axes in relation to the mean length of microtubules drive the system to longitudinal direction of ordering and the opposite combination works towards ordering in the transverse direction. The comparison between the numerical and the simulation results, showed a high qualitative agreement.

Furthermore, regarding the ordering behaviour, we examined the known experimental values for the unbinding rate and the short-axis to mean length ratio of the fission yeast [49]. The conclusion is that the set of the observed values is indeed able to achieve a bipolar ordering.

The equations for the length density of microtubules that we extracted for the case of a general boundary, were applied numerically for different sites of the centrosome inside an ellipse. In this way, we examined the influence of the nucleation position on the length distribution of microtubules. The results showed a high impact of the nucleation site inside the cell on the form of the microtubule distribution.

After the investigation of centrosomal microtubule organization, we proceeded with examining non-centrosomal microtubules. The latter are nucleated from non-centrosomal nucleation sites in most differentiated cells, where they form arrays that are specific to the cell-type. We regarded a large number of nucleation sites that were homogeneously distributed inside an elliptical cell. Then, we defined an order parameter that took into consideration each nucleation site and applied numerically the equation for the length distribution of the stalled microtubules. The implementation of the Monte Carlo integration technique bypassed the complexity of the calculations. The results revealed a similar effect of the cell shape and the unbinding rate from the boundary on ordering of microtubules with the case of a single centrosomal site.

Except of the microtubules length density we investigated their number 
density, too. This was necessary as the distribution of microtubules will be of main interest in the next chapters. By applying numerically the extracted equations of our model, we found that ordering is dominated by the shortest axis and, so, it is transverse. This was an expected result, as the microtubule distribution is exponential in nature, and hence strongly weighted towards shorter lengths.

In a future work our model could be used for the examination of other cell shapes than the elliptical. Furthermore, the stochastic simulations could be extended to $3 \mathrm{D}$ space instead of the $2 \mathrm{D}$ one.

\subsubsection{Sliding on the cell boundary}

In Chapter 4 we investigated the spatial organization of microtubules, which is caused by their dynamic interaction with the cell boundary. The microtubules, unlike the described ones as 'stalled' in Chapter 3, keep growing when they hit the cell boundary and a pushing force is acting on them by the boundary.

The pushing forces have a dynamic character, as they enclose effects like the thermal fluctuations, the microscopic growth process and the Hookean 'stored' length of microtubules. At non-spherical cells these forces lead to 'sliding' of microtubules along the cell boundary, which has been observed experimentally in vitro 55.

In the present study we developed a 'boundary-sliding' model in order to predict the motion of microtubules that slide along the cell boundary. Our model considers the dynamic nature of the pushing forces, unlike other currently developed models. This leads to a significant difference in the system's behaviour, as a much stronger longitudinal ordering is enabled.

In the analysis we didn't consider the presence of pulling forces at the boundary since we worked with fixed centrosomes, and so forces transmitted to the centrosome are not salient. We only considered pushing forces because they modulate the dynamics. The effect of pulling forces would be to stabilize microtubules further, but this can effectively accommodated e.g. by regarding a weaker spring constant.

By considering a cylindrical symmetry, we derived the evolution equations for the sliding microtubules. Due to the complexity of the equations, we regarded an ellipsoid of revolution which was a small perturbation to a sphere and we proceeded with the analysis. The analytical solution revealed the establishment of a bipolar longitudinal ordering of the bound 
microtubules to the cell boundary. This is a key conclusion showing that the sliding effect enables the microtubule distribution along the long axis.

By applying 2D stochastic simulations to an ellipse with a centrosome at its center, we verified the existence of a longitudinal ordering direction of the sliding microtubules. Furthermore, we investigated the role of the sliding friction by examining the cases of a high and a low friction coefficient. The simulations showed that the low friction works toward the bipolar longitudinal ordering.

An object of a future work could be the possible rearrangement of microtubule positions at the cell boundary due to the buckling mechanism. However, the increase in model complexity of the latter would be significant.

\subsubsection{Positioning the mitotic spindle}

The mitotic spindle is a structure that plays a crucial role in cell division, as it segregates the chromosomes. In chapter 5 we developed a model that predicts the positioning of the mitotic spindle inside a cell boundary. Unlike the currently developed models, our model considers the dynamic nature of the pushing forces acting on microtubules when they hit the cell boundary. This dynamics is represented by the force-production mechanism of the "stored length".

We considered the spindle as a rigid body that is moving in the viscous cytoplasm. We regarded pulling forces acting on microtubules at the boundary and we considered no sliding effect to take place. The pulling forces are known to play a role in vivo by regulating the cortical microtubule dynamics [22]. The motion of the spindle and its centrosomes enabled us to study the role of the pulling forces on the robustness and accuracy of the spindle positioning.

By applying 2D stochastic simulations, we determined the spindle motion and its position in the steady state. A key result of our analysis was that the mitotic spindle seeks the long axis of the ellipse to orientate by. Furthermore, by examining the fluctuations of the spindle orientation, we revealed the crucial role that the pulling forces play in the stabilization of the spindle. The main conclusion is that the orientation mechanism becomes more precise when pulling forces are present. Moreover, by changing the dimensions ratio of a $2 \mathrm{D}$ cell shape, we found that a transition between two stable orientation states exists. 
In a future work we could analyze other cell shapes and extend the simulation analysis to the $3 \mathrm{D}$ space.

\subsection{Microtubule-based cell polarity}

\subsubsection{Spherical cell}

Cell polarity, which is created by the asymmetric distribution of polarity molecules at the cell boundary, is crucial for many developmental processes in biology. The investigated spatial organization of microtubules induced by their dynamics and the cell shape forms the basis for the establishment of polarity in animal cells. It is the propensity of microtubules to bind and transport proteins to the cell membrane that modifies this spatial organization and leads to cell polarization.

In Chapter 6, we followed a synthetic biology approach in order to generate a spontaneous and persistent polarity in a spherical cell-like environment. To that end, we developed a minimal model based on dynamic microtubules and a single mobile molecular component. The only feedback mechanism we imposed is that the stabilization of the transporting structures (microtubules) at the membrane increases with the local membrane density of the transported molecules (polarity factors).

Using a bifurcation analysis we proved that our model supports a spontaneous unipolar symmetry broken-state with a following steady state persistence. The analytical results were verified by $2 \mathrm{D}$ simulation results. A further explicit analysis revealed the existence of 4 parameters-quantities that govern the behavior of the model. So, there is a parameter which regulates the competitive advantage of stabilized microtubules to recruit more stabilizing polarity factors, a parameter that determines the extent to which polarity factors once inserted to the membrane remain localized, a parameter controlling the steepness of the switch that distinguishes microtubules stabilized at the membrane from non-stabilized ones and a parameter controlling the availability of polarity factors at the membrane. We argue that these 4 quantities are universal for a class of polarization mechanisms that rely on the autocatalysis of localized insertion.

Although the model we proposed does not correspond to any presently known polarity mechanism in vivo, it is fully based on feasible molecular roles. The role of microtubule-mediated transport in maintaining cell polar- 
ity is well established in fission yeast, where the polarity factors Tea1/Tea4 are transported through association with microtubule plus-end tracking (+TIPs) proteins such as Mal3 and the kinesin Tea2 to ensure the polar localization of cell growth [104]. Recently, Recouvreux et. al. [105] have deconstructed this system, by using a explicitly engineered chimeric complex using the membrane binding domain of Pom1 coupled to Mal3. This minimal system also displays clear polar enrichment. This also shows that using +TIPs, of which a large number have been identified through the work of Akhmanova and others [106, 107], is an alternative to more classical plus-end transporters such as the kinesin family of motor proteins 83 . Perhaps the most crucial part of our mechanism is the ability to stabilize microtubules at the membrane. Here there is a recent work that shows that Agrin mediates the localized capture of microtubules and subsequent stabilization by Clasp2 at the synaptic membrane of neuromuscular cells [84. Similarly, the actin binding protein Moesin has been shown to directly bind to microtubules and stabilize them, albeit in the cortex and not at the membrane proper.

On the whole, the main conclusion to be drawn from our analysis, is the possibility of setting up a feasible and robust cell polarity mechanism. This may, at the very least, form the basis of a biochemical reconstitution approach to set up polarity in a minimal cell-like environment, such as lipid bilayer-enclosed microvolumes containing purified and/or engineered protein components. Steps in this direction are currently actively pursued e.g. by the Dogterom lab [108, 109].

In our model we assumed a non-limit availability of tubulin into a finitevolume geometry. However, applying an extended formalism of the microtubule dynamics we proved the robustness of our model against finite size effects on the number and length of microtubules. Interesting future work would be to assume a finite tubulin pool and proceed with simulation analysis in order to verify our analytical results.

Another interesting future study would be to extend our approach to other mechanisms of cell polarity generation that involve cytoskeletal polymers. A prime example is the polarization mechanism in budding yeast which marks the position of the future daughter cell, in which actin plays a role in recruiting polarization factors to the cell membrane [110]. 


\subsubsection{Non-spherical cell}

In Chapter 7 we investigated the establishment of polarity to an elliptical cell. The elliptical boundaries can support the sliding effect, as the pushing forces acting on the microtubules are not vertical to the tangential direction of the cell boundary. So, it is the interaction between the cell shape, the polarity mechanism and the sliding effect that determines the microtubule organization in non-spherical cells. The present study revealed the relative influence of these three factors.

We began our approach with stochastic simulations for the case of an elliptical cell. Surprisingly, we found that the polarization mechanism dominates over the influence of the cell shape or the sliding effect. A win of the shape would be the domination of the bipolar transverse ordering when the sliding is absent or the domination of the bipolar longitudinal ordering when we include sliding independently of the system parameters. Instead of this, we observed the establishment of a unipolar phase at a transverse or a longitudinal axis end for a certain range of the total number of polarity factors.

In order to verify the presence of the unipolar phase, we formulated analytically a model with the strongest possible bipolar bias. A bifurcation analysis proved that indeed a stable unipolar state can exist. In this way, the main conclusion of Chapter 7 was established, that geometrical cues are not strong enough to overcome the intrinsic monopolarity of the developed polarization mechanism.

In a future work, it would be interesting to set up polarity in vivo in an elliptical cell-like environment, by following a reconstitution approach. To this end, we could address the question about what ingredients are necessary to achieve a true stabilized bipolar polarization. Then, a selective establishment of a unipolar or bipolar microtubule ordering in a non-spherical cell could be possible. 


\section{Bibliography}

[1] E-Cell project.

[2] Jonathan R Karr, Koichi Takahashi, and Akira Funahashi. The principles of whole-cell modeling. Current Opinion in Microbiology, $27: 18-24$, oct 2015 .

[3] Leland H. Hartwell, John J. Hopfield, Stanislas Leibler, and Andrew W. Murray. From molecular to modular cell biology. Nature, 402(6761supp):C47-C52, dec 1999.

[4] E Fuchs and K Weber. Intermediate Filaments: Structure, Dynamics, Function and Disease. Annual Review of Biochemistry, 63(1):345382, jun 1994.

[5] Daniel A Fletcher and R Dyche Mullins. Cell mechanics and the cytoskeleton. Nature, 463(7280):485-92, jan 2010.

[6] J.-M. Trifaró, S. Gasman, and L. M. Gutiérrez. Cytoskeletal control of vesicle transport and exocytosis in chromaffin cells. Acta Physiologica, 192(2):165-172, nov 2007.

[7] Joost van Mameren, Karen C. Vermeulen, Fred Gittes, and Christoph F. Schmidt. Leveraging Single Protein Polymers To Measure Flexural Rigidity. The Journal of Physical Chemistry B, 113(12):3837-3844, mar 2009.

[8] Nobutaka Hirokawa. Kinesin and Dynein Superfamily Proteins and the Mechanism of Organelle Transport. Science, 279(5350):519-526, jan 1998.

[9] Marileen Dogterom and Stanislas Leibler. Physical aspects of the growth and regulation of microtubule structures. Physical review letters, 70(9):1347-1350, 1993. 
[10] David Sept. Microtubule polymerization: one step at a time. Current biology : $C B, 17(17): \mathrm{R} 764-6$, sep 2007.

[11] Hugo Bowne-Anderson, Marija Zanic, Monika Kauer, and Jonathon Howard. Microtubule dynamic instability: A new model with coupled GTP hydrolysis and multistep catastrophe. BioEssays, 35(5):452461, may 2013.

[12] Jingchao $\mathrm{Wu}$ and Anna Akhmanova. Microtubule-Organizing Centers. Annual Review of Cell and Developmental Biology, 33(1):51-75, oct 2017.

[13] Martine Pastuglia and David Bouchez. Molecular encounters at microtubule ends in the plant cell cortex. Current Opinion in Plant Biology, 10(6):557-563, dec 2007.

[14] T Murata, S Sonobe, T I Baskin, S Hyodo, S Hasezawa, T Nagata, $\mathrm{T}$ Horio, and M Hasebe. Microtubule-dependent microtubule nucleation based on recruitment of big gamma-tubulin in higher plants. Nature Cell Biol., 7:961-968, 2005.

[15] J Chan, A Sambade, G Calder, and C Lloyd. Arabidopsis Cortical Microtubules Are Initiated along, as Well as Branching from, Existing Microtubules. Plant Cell, 21:2298-2306, 2009.

[16] David W Ehrhardt and Sidney L Shaw. Microtubule Dynamics And Organization In The Plant Cortical Array. Ann. Rev. of Plant Biol., 57:859-875, 2006.

[17] R Dixit and R Cyr. Encounters between Dynamic Cortical Microtubules Promote Ordering of the Cortical Array through AngleDependent Modifications of Microtubule Behavior. Plant Cell, 16:3274-3284, 2004.

[18] Simon H Tindemans, Rhoda J Hawkins, and Bela M Mulder. Survival of the Aligned: Ordering of the Plant Cortical Microtubule Array. Phys. Rev. Lett., 104(5):58103, feb 2010.

[19] Alex Paradez, Amanda Wright, and David W Ehrhardt. Microtubule cortical array organization and plant cell morphogenesis. Current Opinion in Plant Biology, 9(6):571-578, dec 2006. 
[20] Bandan Chakrabortty, Ikram Blilou, Ben Scheres, and Bela M. Mulder. A computational framework for cortical microtubule dynamics in realistically shaped plant cells. PLOS Computational Biology, 14(2):e1005959, feb 2018.

[21] E E Deinum, S H Tindemans, and B M Mulder. Taking directions: The role of microtubule-bound nucleation in the self-organization of the plant cortical array. Phys. Biol., 8:56002, 2011.

[22] Liedewij Laan, Nenad Pavin, Julien Husson, Guillaume RometLemonne, Martijn van Duijn, Magdalena Preciado López, Ronald D Vale, Frank Jülicher, Samara L Reck-Peterson, and Marileen Dogterom. Cortical Dynein Controls Microtubule Dynamics to Generate Pulling Forces that Position Microtubule Asters. Cell, 148(3):502-514, 2012.

[23] Francis J McNally. Mechanisms of spindle positioning. The Journal of cell biology, 44(2):131-140, 2013.

[24] Jingchen Li and Hongyuan Jiang. Regulating positioning and orientation of mitotic spindles via cell size and shape. Physical Review E, 97(1):012407, jan 2018.

[25] Carlos Garzon-Coral, Horatiu A Fantana, and Jonathon Howard. A force-generating machinery maintains the spindle at the cell center during mitosis. Science (New York, N.Y.), 352(6289):1124-7, may 2016 .

[26] Stephan W. Grill and Anthony A. Hyman. Spindle Positioning by Cortical Pulling Forces. Developmental Cell, 8(4):461-465, apr 2005.

[27] Home - BaSyC.

[28] Nicolas Le Novère and Dominic P Tolle. Particle-Based Stochastic Simulation in Systems Biology JSBML: a Java library for manipulating SBML View project Impact of Technology on an Organization Performance (Motorbike Industry of Pakistan) View project Dominic P Tolle Particle-Based Stochastic Si. Current Bioinformatics, 1:315$320,2006$. 
[29] Henry T. Schek, III, Melissa K. Gardner, Jun Cheng, David J. Odde, and Alan J. Hunt. Microtubule Assembly Dynamics at the Nanoscale. Current biology : $C B, 17(17): 1445$, sep 2007.

[30] R J Rubin. Mean lifetime of microtubules attached to nucleating sites. Proceedings of the National Academy of Sciences of the United States of America, 85(2):446-8, jan 1988.

[31] Sidney L Shaw, Jacques Dumais, and Sharon R Long. Cell Surface Expansion in Polarly Growing Root Hairs of Medicago truncatula. Plant Physiology, 124(3):959-970, 2000.

[32] Alexander R Paredez, Christopher R Somerville, and David W Ehrhardt. Visualization of Cellulose Synthase Demonstrates Functional Association with Microtubules. Science, 312(5779):1491-1495, 2006.

[33] Ryan Gutierrez, Jelmer J Lindeboom, Alex R Paredez, Anne Mie C Emons, and David W Ehrhardt. Arabidopsis cortical microtubules position cellulose synthase delivery to the plasma membrane and interact with cellulose synthase trafficking compartments. Nature Cell Biol., 11:797-806, 2009.

[34] A Desai and T J Mitchison. Microtubule polymerization dynamics. Annu. Rev. Cell Dev. Biol., 13:83-117, jan 199.

[35] J F Allard, G O Wasteneys, and E N Cytrynbaum. Mechanisms of self-organization of cortical microtubules in plants revealed by computational simulations. Mol. Biol. Cell, 21:278-286, 2010.

[36] E C Eren, R Dixit, and N Gautam. A 3D Computer Simulation Model Reveals the Mechanisms for Self-Organization of Plant Cortical Microtubules into Oblique Arrays. Mol. Biol. Cell, 21:2674-2684, 2010 .

[37] R J Hawkins, S H Tindemans, and B M Mulder. Model for the orientational ordering of the plant microtubule cortical array. Phys. Rev. E, 82:11911, 2010.

[38] Jens Luders and Tim Stearns. Microtubule-organizing centres: a reevaluation. Nature Rev. Mol. Cell Biol., 8:161-167, 2007. 
[39] M. Nakamura, Ehrhardt.D.W., and T. Hashimoto. Microtubule and katanin-dependent dynamics of microtubule nucleation complexes in the acentrosomal Arabidopsis cortical array. Nature Cell Biol., 12:1064-1070, 2010.

[40] David H Burk, Ruiqin Zhong, and Zheng-Hua Ye. The Katanin Microtubule Severing Protein in Plants. Journal of Integrative Plant Biology, 49(8):1174-1182, 2007.

[41] Eva E Deinum and Bela M Mulder. Modelling the role of microtubules in plant cell morphology. Current Opinion in Plant Biology, 44(6):688-692, 2013.

[42] S H Tindemans. Biomolecular design elements : cortical microtubules and DNA-coated colloids. PhD thesis, Wageningen University, 2009.

[43] Clare M Waterman-Storer and E D Salmon. Microtubule dynamics: Treadmilling comes around again. Current biol., 7:R369-R372, 1997.

[44] Juan Manuel Gomez, Lyubov Chumakova, Natalia A. Bulgakova, and Nicholas H. Brown. Microtubule organization is determined by the shape of epithelial cells. Nature Communications, 7:1-13, 2016.

[45] Eckhard Mandelkow and Eva-Maria Mandelkow. Microtubules and microtubule-associated proteins. Current Opinion in Cell Biology, 7:72-81, 1995.

[46] Andrew Muroyama and Terry Lechler. Microtubule organization, dynamics and functions in differentiated cells. Development, 144(17):3012-3021, sep 2017.

[47] F. Bartolini and G. G. Gundersen. Generation of noncentrosomal microtubule arrays. Journal of Cell Science, 119(20):4155-4163, oct 2006.

[48] Ariana D. Sanchez and Jessica L. Feldman. Microtubule-organizing centers: from the centrosome to non-centrosomal sites. Current Opinion in Cell Biology, 44:93-101, 2017. 
[49] P T Tran, L Marsh, V Doye, S Inoué, and F Chang. A mechanism for nuclear positioning in fission yeast based on microtubule pushing. Journal of Cell Biology, 153(2):397-411, 2001.

[50] Marileen Dogterom and Bernard Yurke. Measurement of the forcevelocity relation for growing microtubules. Science, 278(5339):856860, 1997.

[51] Bela M Mulder. Microtubules interacting with a boundary : mean length and mean first-passage times. Phys. Rev. E, 86(1):011902 1-11, 2012.

[52] Xiaolei Su, Hugo Arellano-Santoyo, Didier Portran, Jeremie Gaillard, Marylin Vantard, Manuel Thery, and David Pellman. Microtubule sliding activity of a kinesin- 8 promotes spindle assembly and spindle length control. Nature Cell Biology, 15(2):948-957, 2013.

[53] J Vogel, B Drapkin, J Oomen, D Beach, K Bloom, and M Snyder. Phosphorylation of gamma-tubulin regulates microtubule organization in budding yeast. Developmental cell, 1(5):621-31, nov 2001.

[54] Michiel. Hazewinkel. Encyclopaedia of mathematics. Springer-Verlag, 2002.

[55] Liedewij Laan, Sophie Roth, and Marileen Dogterom. End-on microtubule-dynein interactions and pulling-based positioning of microtubule organizing centers. Cell Cycle, 11(20):3750-3757, 2012.

[56] Iva M Tolic-Nørrelykke, Leonardo Sacconi, Genevieve Thon, and Francesco S Pavone. Positioning and Elongation of the Fission Yeast Spindle by Microtubule-Based Pushing. Current biol., 14, 2004.

[57] Marcel E Janson, Mathilde E de Dood, and Marileen Dogterom. Dynamic instability of microtubules is regulated by force. Current Opinion in Cell Biology, 44(6):1029-1034, 2003.

[58] Dietrich Foethke, Tatyana Makushok, Damian Brunner, and François J Nédélec. Force- and length-dependent catastrophe activities explain interphase microtubule organization in fission yeast. Current Opinion in Cell Biology, 44(241):1-6, 2009. 
[59] Rui Ma, Liedewij Laan, Marileen Dogterom, Nenad Pavin, and Frank Jülicher. General theory for the mechanics of confined microtubule asters. New Journal of Physics, 16(1):13018, 2014.

[60] Juliane Teapal. Microtubule-based positioning mechanisms. $\mathrm{PhD}$ thesis, Wageningen University, 2014.

[61] Janko Kajtez, Anastasia Solomatina, Maja Novak, Bruno Polak, Kruno Vukušić, Jonas Rüdiger, Gheorghe Cojoc, Ana Milas, Ivana Šumanovac Šestak, Patrik Risteski, Federica Tavano, Anna H. Klemm, Emanuele Roscioli, Julie Welburn, Daniela Cimini, Matko Glunčić, Nenad Pavin, and Iva M. Tolić. Overlap microtubules link sister k-fibres and balance the forces on bi-oriented kinetochores. $\mathrm{Na}$ ture Communications, 7(1):10298, dec 2016.

[62] Iva M. Tolić. Mitotic spindle: kinetochore fibers hold on tight to interpolar bundles. European Biophysics Journal, 47(3):191-203, apr 2018.

[63] Yuki Hara and Akatsuki Kimura. An allometric relationship between mitotic spindle width, spindle length, and ploidy in Caenorhabditis elegans embryos. Molecular biology of the cell, 24(9):1411-9, may 2013.

[64] Alex J Levine, T B Liverpool, and F C Mackintosh. Mobility of extended bodies in viscous films and membranes. Phys. Rev. E, 69:021503, 2003.

[65] Claire E. Walczak and Rebecca Heald. Mechanisms of Mitotic Spindle Assembly and Function. In International review of cytology, volume 265, pages 111-158. Elsevier, 2008.

[66] Yuta Shimamoto, YusukeT. Maeda, Shin'ichi Ishiwata, AlbertJ. Libchaber, and TarunM. Kapoor. Insights into the Micromechanical Properties of the Metaphase Spindle. Cell, 145(7):1062-1074, jun 2011.

[67] Albert Einstein. On the Motion of Small Particles Suspended in Liquids at Rest Required by the Molecular-Kinetic Theory of Heat * . Annalen der Physik, 17:549-560, 1905. 
[68] B. Cichocki, B. U. Felderhof, K. Hinsen, E. Wajnryb, and J. Bl/awzdziewicz. Friction and mobility of many spheres in Stokes flow. The Journal of Chemical Physics, 100(5):3780-3790, mar 1994.

[69] Beatriz Carrasco and José García de la Torre. Hydrodynamic Properties of Rigid Particles: Comparison of Different Modeling and Computational Procedures. Biophysical Journal, 76(6):3044-3057, jun 1999.

[70] Kaiyuan Yang, Chen Lu, Xiaodan Zhao, and Ryo Kawamura. From bead to rod: Comparison of theories by measuring translational drag coefficients of micron-sized magnetic bead-chains in Stokes flow. PLOS ONE, 12(11):e0188015, nov 2017.

[71] S. Broersma. Viscous force and torque constants for a cylinder. The Journal of Chemical Physics, 74(12):6989-6990, jun 1981.

[72] A. Ortega and J. Garcia de la Torre. Hydrodynamic properties of rodlike and disklike particles in dilute solution. The Journal of Chemical Physics, 119(18):9914-9919, nov 2003.

[73] Barry J Thompson. Cell polarity: models and mechanisms from yeast, worms and flies. Development, 140(1):13-21, 2013.

[74] Alex Mogilner, Jun Allard, and Roy Wollman. Cell Polarity: Quantitative Modeling as a Tool in Cell Biology. Science, 336(6078):175-179, 2012.

[75] Alexandra Jilkine and Leah Edelstein-Keshet. A comparison of mathematical models for polarization of single eukaryotic cells in response to guided cues. PLoS computational biology, 7(4):e1001121, 2011.

[76] Angela H Chau, Jessica M Walter, Jaline Gerardin, Chao Tang, and Wendell A Lim. Designing Synthetic Regulatory Networks Capable of Self-Organizing Cell Polarization. Cell, 151(2):320-332, 2012.

[77] Rong Li and Gregg G Gundersen. Beyond polymer polarity: how the cytoskeleton builds a polarized cell. Current Opinion in Cell Biology, 9(11):860-873, nov 2008.

[78] Fred Chang and Sophie G Martin. Shaping fission yeast with microtubules. Cold Spring Harbor perspectives in biology, 1(1):a001347, 2009. 
[79] Eugenio Marco, Roland Wedlich-Soldner, Rong Li, Steven J Altschuler, and Lani F Wu. Endocytosis optimizes the dynamic localization of membrane proteins that regulate cortical polarity. Cell, 129(2):411-422, 2007.

[80] Steven J Altschuler, Sigurd B Angenent, Yanqin Wang, and Lani F $\mathrm{Wu}$. On the spontaneous emergence of cell polarity. Nature, 454(7206):886-889, 2008.

[81] Alexandra Jilkine, Sigurd B Angenent, Lani F Wu, and Steven J Altschuler. A density-dependent switch drives stochastic clustering and polarization of signaling molecules. PLoS Computational Biology, 7(11):e1002271, 2011.

[82] Tina Freisinger, Ben Klünder, Jared Johnson, Nikola Müller, Garwin Pichler, Gisela Beck, Michael Costanzo, Charles Boone, Richard A Cerione, Erwin Frey, and Others. Establishment of a robust single axis of cell polarity by coupling multiple positive feedback loops. $\mathrm{Na}$ ture Communications, 4:1807, 2013.

[83] Nobutaka Hirokawa, Yasuko Noda, Yosuke Tanaka, and Shinsuke Niwa. Kinesin superfamily motor proteins and intracellular transport. Nature reviews. Molecular cell biology, 10(10):682-696, 2009.

[84] Nadine Schmidt, Sreya Basu, Stefan Sladecek, Sabrina Gatti, Jeffrey van Haren, Susan Treves, Jan Pielage, Niels Galjart, and Hans Rudolf Brenner. Agrin regulates CLASP2-mediated capture of microtubules at the neuromuscular junction synaptic membrane. The Journal of cell biology, 198(3):421-437, 2012.

[85] Sara Solinet, Kazi Mahmud, Shannon F Stewman, Khaled Ben El Kadhi, Barbara Decelle, Lama Talje, Ao Ma, Benjamin H Kwok, and Sébastien Carreno. The actin-binding ERM protein Moesin binds to and stabilizes microtubules at the cell cortex. The Journal of cell biology, 202(2):251-260, 2013.

[86] Mikiya Otsuji, Shuji Ishihara, Carl Co, Kozo Kaibuchi, Atsushi Mochizuki, and Shinya Kuroda. A mass conserved reaction-diffusion system captures properties of cell polarity. PLoS computational biology, 3(6):e108, 2007. 
[87] Andrew B Goryachev and Alexandra V Pokhilko. Dynamics of Cdc42 network embodies a Turing-type mechanism of yeast cell polarity. FEBS letters, 582(10):1437-1443, 2008.

[88] M et al Milani. Differential Two Colour X-ray Radiobiology of Membrane/Cytoplasm Yeast Cells. Technical report, TMR Large-Scale Facilities Access Programme,University of Milan, 1998.

[89] Michelle Piehl, U Serdar Tulu, Pat Wadsworth, and Lynne Cassimeris. Centrosome maturation: measurement of microtubule nucleation throughout the cell cycle by using GFP-tagged EB1. Proceedings of the National Academy of Sciences of the United States of America, 101(6):1584-1588, feb 2004.

[90] Mark J. Schnitzer, Koen Visscher, and Steven M. Block. Force production by single kinesin motors. Nature Cell Biology, 2(10):718-723, oct 2000 .

[91] Konstadinos Moissoglu, Boris M Slepchenko, Nahum Meller, Alan F Horwitz, and Martin A Schwartz. In Vivo Dynamics of Rac-Membrane Interactions. Molecular Biology of the Cell, 17(June):2770-2779, 2006.

[92] B Buendia, G Draetta, and E Karsenti. Regulation of the microtubule nucleating activity of centrosomes in Xenopus egg extracts: role of cyclin A-associated protein kinase. The Journal of cell biology, 116(6):1431-1442, mar 1992.

[93] Bruce Alberts, Alexander Johnson, Julian Lewis, Martin Raff, Keith Robers, and Peter Walter. Molecular Biology of the Cell. Garland Publishing, 3rd edition, 2002.

[94] J D McGhee and P H V Hippel. Theoretical Aspects of Dna-Protein Interactions - Cooperative and Non-Cooperative Binding of Large Ligands to A One-Dimensional Homogeneous Lattice. Journal of Molecular Biology, 86(2):469-489, 1974.

[95] Daniel R Gestaut, Beth Graczyk, Jeremy Cooper, Per O Widlund, Alex Zelter, Linda Wordeman, Charles L Asbury, and Trisha N Davis. Phosphoregulation and depolymerization-driven movement of 
the Dam1 complex do not require ring formation. Nature cell biology, 10(4):407-414, apr 2008.

[96] Matthew C Good, Michael D Vahey, Arunan Skandarajah, Daniel A Fletcher, and Rebecca Heald. Cytoplasmic Volume Modulates Spindle Size During Embryogenesis. Science, 342(6160):856-860, 2013.

[97] Marileen Dogterom and Thomas Surrey. Microtubule organization in vitro. Current Opinion in Cell Biology, 25(1):23-29, 2013.

[98] John D. Wehr, Robert G. Sheath, and John Patrick Kociolek. Freshwater algae of North America : ecology and classification. Academic Press, 2015.

[99] Ovalocytes/elliptocytes. https://www.labce.com/spg469627_ ovalocyteselliptocytes.aspx.

[100] D Aprianti, F Haryanto, A Purqon, S N Khotimah, and S Viridi. Study of budding yeast colony formation and its characterizations by using circular granular cell. Journal of Physics: Conference Series, 694(1):012079, mar 2016.

[101] Nria Taberner, Andries Lof, Sophie Roth, Dimitry Lamers, Hans Zeijlemaker, and Marileen Dogterom. . in vitro systems for the study of microtubule-based cell polarity in fission yeast. In Jennifer Ross and Wallace F. Marshall, editors, Building a cell from its component parts, volume 128 of Methods in Cell Biology, chapter 1. Elsevier, 2015 .

[102] Scott A. Socolofsky. Mixing, Transport and Transformation: Instantaneous Point-source Solution. University of Karlsrue, Institute of Hydromechanics, 2002.

[103] John Burkardt. Ziggurat Random Number Generator (RNG). https://people.sc.fsu.edu/ jburkardt/f_src/ziggurat/ziggurat.html.

[104] J Mata and P Nurse. tea1 and the microtubular cytoskeleton are important for generating global spatial order within the fission yeast cell. Cell, 89(6):939-49, jun 1997. 
[105] Pierre Recouvreux, Thomas R Sokolowski, Aristea Grammoustianou, Pieter Rein ten Wolde, and Marileen Dogterom. Chimera proteins with affinity for membranes and microtubule tips polarize in the membrane of fission yeast cells. Proceedings of the National Academy of Sciences, 113(7):1811-1816, 2016.

[106] Anna Akhmanova and Casper C Hoogenraad. Microtubule plus-endtracking proteins: mechanisms and functions. Current Opinion in Cell Biology, 17(1):47-54, 2005.

[107] Niels Galjart. Plus-End-Tracking Proteins and Their Interactions at Microtubule Ends. Current Biology, 20(12):R528 - R537, 2010.

[108] Núria Taberner, Georges Weber, Changjiang You, Roland Dries, Jacob Piehler, and Marileen Dogterom. Reconstituting functional microtubule-barrier interactions. Methods in cell biology, 120:69-90, 2014.

[109] Núria Taberner, Andries Lof, Sophie Roth, Dimitry Lamers, Hans Zeijlemaker, and Marileen Dogterom. In vitro systems for the study of microtubule-based cell polarity in fission yeast. Methods in Cell Biology, 128:1-22, 2015.

[110] David Pruyne, Aster Legesse-Miller, Lina Gao, Yuqing Dong, and Anthony Bretscher. MECHANISMS OF POLARIZED GROWTH AND ORGANELLE SEGREGATION IN YEAST. Annual Review of Cell and Developmental Biology, 20(1):559-591, nov 2004. 


\section{Summary}

Living cells are highly complex systems and their study encompasses a wide range of challenging questions. Broadly speaking, two different modelling strategies have been developed to date to confront these challenges. The first is the whole-cell modelling approach, which is based on storing and processing information about metabolic pathways, gene regulation and expression in electronic platforms. However, this modelling approach by failing to a large extent to consider the spatial dynamics of cellular molecules and structures, cannot analyze many basic physical and biochemical effects, which are spatially dependent. For these types of effects the so-called modular approach to cell biology has proved extremely effective. This approach is based on distinguishing and then studying in isolation finite sets of intracellular components - known as functional modules - that perform distinct biological functions. In the present thesis, we adopt this latter approach and study a number of cellular modules, with the common feature that they all relate to the dynamics of microtubules.

Microtubules are the stiffest polymers of the cytoskeleton, being effectively rigid on the typical scale of cells. A key feature of microtubules is their highly dynamic character determined by their stochastic switching between states of growth and of shrinkage, which is an intrinsic property known as dynamic instability. The microtubule cytoskeleton plays many important roles in the cell. Among the various tasks that it fulfills are controlling the overall cell shape and their role in the transport of vesicles or other molecular components to the cell membrane leading to an asymmetric spatial organization known as cell polarization.

The first microtubule-based cellular module that we study is the cortical microtubule array in growing interphase plant cells. The plant cortical microtubules are nucleated from nucleation complexes that are mainly attached to pre-existing microtubules. Their attachment to the membrane creates an effectively $2 \mathrm{D}$ environment for the observed collisional interac- 
tions between them. In Chapter 2 we incorporate the microtubule-bound anisotropic nucleation to a theoretical framework previously developed. A bifurcation analysis confirms the co-alignment of the branched nucleations with the parent microtubules as the main determinant of the transition location to order. A numerical analysis shows the robustness of our mean-field theory approach after comparing with particle-based simulation results. Our analytical model confirms the strong self-consistent positive feedback on microtubule ordering. Furthermore, a numerical analysis shows that by increasing branched vs background nucleation, we get a wider regime of the microtubule dynamical parameters for which the system is ordered. This means that the ordered state becomes more robust as it can resist higher variation of the system parameters, which is potentially biologically significant.

The second cellular module that we study is the interphase radial microtubule array in animal cells. This is an array of microtubules that are nucleated by a single centrosome and extend radially towards the cell boundary. In Chapter 3 we develop a 3D-model for centrosomal microtubules that can stall at the cell boundary and we investigate their spatial distribution. We derive the equations that describe the distribution of microtubules for a general form of a boundary. We show that in the presence of an axial symmetry the behaviour of our model is not very sensitive to a change of dimension. A numerical analysis applied to the case of an ellipse with a centrosome fixed at its center, reveals the existence of two directions of ordering. One is longitudinal and the other is transverse, each one dominating at a different regime of the system parameters. Furthermore, we investigate the distribution of non-centrosomal microtubules nucleated homogeneously inside an ellipse. A numerical analysis reveals a similar effect of the cell shape and the system parameters on ordering of microtubules with the case of a single centrosomal site.

In Chapter 4 we extend our study on the radial microtubule array by regarding its dynamic interaction with the cell boundary. Based on a Hookean pushing force acting on microtubules by the boundary, we build a boundary-sliding model in order to predict the motion of microtubules that slide along the cell membrane. By considering a cylindrical symmetry, we derive the evolution equations for the sliding microtubules. The analytical solution for an ellipsoid of revolution which is a small perturbation to a sphere reveals the establishment of a bipolar longitudinal ordering of the 
boundary microtubules. The distribution along the long axis is verified by 2D stochastic simulations applied to an ellipse.

The third cellular module that we study is the localization dynamics of the mitotic spindle, which plays a crucial role in cell division. The interaction of microtubules with the cell boundary generates forces which lead to the positioning of the mitotic spindle so that the correct spatial segregation of the daughter chromosomes is enabled. In Chapter 4 we develop a model that based on pushing and pulling forces acting on the astral microtubules of the mitotic spindle, predicts its positioning inside a cell boundary. By applying 2D stochastic simulations to an ellipse we find that the spindle seeks the long axis to orientate by. The main conclusion extracted is that the orientation mechanism becomes more precise when pulling forces are present. Furthermore, by changing the dimensions ratio of a $2 \mathrm{D}$ cell shape, we find that a transition between two stable orientation states exists.

The fourth cellular module that we'll investigate are microtubule-based cell polarization mechanisms. Cell polarization is created by the asymmetric distribution of intracellular components at the cell boundary. The ability of microtubules to bind and selectively transport proteins to the plasma membrane is the property that opens a route to cell polarization. In Chapter 6 we follow a synthetic biology approach in order to generate a spontaneous and persistent polarity in a spherical cell-like environment. To that end, we develop a minimal model based on dynamic microtubules and a single mobile molecular component. The only feedback mechanism we impose is that the stabilization of the transporting microtubules at the membrane increases with the local membrane density of the transported molecules. A bifurcation analysis proves that our model supports a spontaneous unipolar symmetry broken-state with a following steady state persistence. The analytical results are verified by $2 \mathrm{D}$ stochastic simulations. Although the model we propose does not correspond to any presently known polarity mechanism in vivo, it is fully based on feasible molecular roles.

In Chapter 7 we investigate the establishment of polarity to an elliptical cell. We explore the relative influence of the cell shape, the polarity mechanism and the sliding effect on the microtubule organization. Stochastic simulations show that a unipolar phase can exist for a certain range of the number of the polarity components. This is verified analytically by a model with the strongest possible bipolar bias. The main conclusion is that geo- 
metrical cues are not strong enough to overcome the intrinsic unipolarity of the developed polarization mechanism.

The models that we develop in the current thesis lay the foundation for understanding how the spatial organization of microtubules is influenced by the cell shape or polarization mechanisms. Furthermore, they can form the basis of a rational design approach towards using biochemical reconstitution to set up polarity in a minimal cell-like environments. In the concluding Chapter 8 , we discuss some possible directions of future work inspired by the present study. 


\section{Samenvatting}

Levende cellen zijn zeer complexe systemen en hun studie omvat een breed scala aan uitdagende vragen. Tot op heden zijn er in grote lijnen twee strategieën ontwikkeld om deze uitdagingen het hoofd te bieden. De eerste benadering probeert de cel in zijn geheel te modelleren. Deze aanpak is gebaseerd op het opslaan en verwerken van alle beschikbare informatie over metabole reactiepaden en genregulatienetwerken. Zon benadering vereist noodzakelijkerwijs de inzet van grootschalige rekencapaciteit. Deze vorm van modelleren gaat doorgaans echter voorbij aan de ruimtelijke dynamiek van moleculen en structuren in de cel en verwaarloost daarom veel fundamentele fysische- en biochemische effecten, die sterk ruimtelijk afhankelijk zijn. Voor deze klasse van verschijnselen is de zogenaamde modulaire benadering van celbiologie uiterst effectief gebleken. Deze aanpak is gebaseerd op het onderscheiden en vervolgens in isolatie bestuderen van eindige verzamelingen van intracellulaire componenten - bekend als functionele modules - die elk verantwoordelijk zijn voor specifieke biologische functies.

In dit proefschrift volgen we deze laatste aanpak en bestuderen een aantal cellulaire modules met als gemeenschappelijk kenmerk dat ze allemaal betrekking hebben op de dynamiek van microtubuli.

Microtubuli zijn de stijfste polymeren van het cytoskelet, en effectief als rigide te beschouwen op de typische lengteschaal van cellen. Een belangrijk kenmerk van microtubuli is hun zeer dynamische karakter bepaald door hun stochastische omschakeling tussen toestanden van groei en van krimp, een intrinsieke eigenschap bekend als dynamische instabiliteit. Het microtubule cytoskelet speelt een aantal belangrijke rollen in de cel. Onder die verschillende taken is het onderhouden de algehele celvorm en een grote rol in het transport van vesicles (celblaasjes) of andere moleculaire componenten naar het celmembraan. Dit laatste proces kan bijvoorbeeld leiden tot een asymmetrische ruimtelijke organisatie bekend als celpolarisatie.

De eerste op microtubuli gebaseerde cellulaire module die we bestud- 
eren is de zogenaamde corticale microtubule array in groeiende interfase plantencellen. De corticale microtubuli in planten worden genucleëerd door nucleatiecomplexen die voornamelijk gekoppeld aan reeds bestaande microtubuli. Doordat ze gehecht zijn aan de binnenzijde van het celmembraan creëert dit een effectieve 2D-omgeving wat aanleiding is voor de interacties door botsingen, die op hun beurt de oorzaak zijn voor de waargenomen oplijning van de array als geheel.

In Hoofdstuk 2 bestuderen we de invloed van microtubule-gebonden anisotrope nucleatie naar een eerder ontwikkeld theoretisch model. Een bifurcatie-analyse van de geformuleerde theorie bevestigt dat de mate waarin de vertakte nucleaties in dezelfde richting wijzen als de ouderlijke microtubuli de belangrijkste determinant van de locatie van de ordeningsovergang. Een numerieke analyse toont de robuustheid van de door ons gebruikte gemiddelde-veld benadering aan door de uitkomsten te vergelijken met eerdere op deeltjes gebaseerde simulatieresultaten. Ons analytisch model bevestigt zo de sterke, zelfconsistente positieve feedback bij het ordenen van microtubuli. Bovendien laat een numerieke analyse dat zien door het vergroten van vertakte nucleatie ten opzichte van de doorgaans isotrope achtergrond nucleatie, we een breder regime van de dynamische parameters van de microtubule krijgen waarvoor het systeem is geordend. Dit betekent dat de geordende toestand robuuster wordt tegen variatie van de systeemparameters, wat potentieel biologisch significant is.

De tweede cellulaire module die we bestuderen is de interfase radiale microtubule array in dierlijke cellen. Dit is een array van microtubuli die genucleëerd worden door één enkel centrosoom en zich vervolgens radiaal uitstrekken naar de celgrens.

In hoofdstuk 3 ontwikkelen we een 3D-model voor centrosomale microtubuli die geblokkeerd worden op de celgrens en we onderzoeken hun ruimtelijke verdeling. We leiden de vergelijkingen af die de verdeling van microtubuli beschrijven voor een algemene celgeometrie. We laten dat zien dat in de aanwezigheid van een axiale symmetrie het gedrag van ons model niet erg gevoelig is voor een verandering van dimensie. Een numerieke analyse toegepast op het geval van een ellips met één centrosoom in het midden van de cel, laat zien dat er twee mogelijke richtingen van ordening bestaan. De ene is longitudinaal en de andere is transversaal, ieder te induceren in een ander regime van de systeemparameters. Verder onderzoeken wij de verdeling van niet-centrosomale microtubuli die op homogeen verdeelde 
locaties binnen een ellipsvormige cel worden genuceleerd. Een numerieke analyse onthult dat er vergelijkbare effecten van de celvorm en de systeemparameters optreden wat de ordening betreft als eerder gezien bij een enkele centrosomale site.

In Hoofdstuk 4 breiden we ons onderzoek naar de radiale microtubule array uit door de dynamiek van de interactie met de celgrens te beschouwen. We formuleren een op een Hookeaanse veerkracht gebaseerd model dat kan beschrijven hoe tegen het membraan duwende microtubuli kunnen glijden. Voor het cilindrisch symmetrische geval kunnen we de evolutievergelijking van de ruimtelijke verdeling van microtubuli aan het membraan opstellen. Door middel van een analystische perturbatie analyse van het geval van een bijna-sferische omwentelingsellipsoide kunnen we laten zien dat het systeem streeft naar een bipolaire ordening langs de langste as van de cel. Dit effect kan vervolgens geverifieerd worden door stochastische simulaties in een 2dimensionale ellipsvormige cel.

De derde cellulaire module die we bestuderen is de lokalisatiedynamiek van de mitotische spindel, die een cruciale rol speelt in celdeling. De interactie van microtubuli met de celgrens genereert krachten die leiden tot de positionering van de mitotische spindel in de celgeometrie, nodig voor de correcte ruimtelijke segregatie van de dochterchromosomen.

In hoofdstuk 5 ontwikkelen we een model dat gebaseerd is op zowel duwende als trekkende krachten die werken op de zogenaamde astrale microtubuli van de mitotische spindel dat in staat is om de positionering te voorspellen als functie van de celvorm. Door middel van 2D-stochastische simulaties in een ellipsvormige cel, vinden we dat de spil vanzelf de lange as zoekt om zich te oriënteren. De belangrijkste conclusie die we uit deze simulaties halen is dat het oriëntatiemechanisme preciezer wordt wanneer trekkrachten aanwezig zijn. Door vervolgens de lengte-breedte verhouding van de cel te variren van de 2D-celvorm, laten we ook zien we dat een er overgang tussen twee stabiele orintaties bestaat.

De vierde cellulaire module die we onderzoeken zijn celpolarisatiemechanismen gebaseerd op microtubuli. In het algemeen wordt celpolarisatie gekarakteriseerd door een asymmetrische verdeling van intracellulaire componenten aan de celgrens. Het vermogen van microtubuli om eiwitten te binden en vervolgens selectief te transporteren naar het celmembraan is de cruciale eigenschap die een mogelijke route naar celpolarisatie opent.

In Hoofdstuk 6 volgen we een synthetische biologie benadering om de 
vraag te beantwoorden hoe we een spontane en persistente polariteit kunnen genereren in een strikt bolvormige cel. Daartoe ontwikkelen we een minimaal model op basis van dynamische microtubuli en één enkele mobiele moleculaire component. Het enige feedbackmechanisme dat wij veronderstellen is dat de stabilisatie van de transporterende microtubuli aan het membraan toe neemt met de lokale dichtheid van de getransporteerde moleculen. Een bifurcatie-analyse bewijst dat ons model ondersteuning biedt voor het bestaan van een unipolaire symmetrie-gebroken toestand in steady-state. Deze analytische resultaten worden vervolgens geverifieerd door 2D-stochastische simulaties. Hoewel het voorgestelde model niet overeenkomt met enig op dit moment bekend polariteitsmechanisme in vivo, is het volledig gebaseerd op realistische moleculaire rollen en parameterwaarden.

In Hoofdstuk 7 tenslotte, onderzoeken we de invloed van celgeometrie op het eerder geintroduceerde polarisatiemechanisme in een elliptische cel. We verkennen hierbij zowel de rol van de celvorm op zich als de invloed van een het krachtgedreven model voor het glijden van microtubuli gentroduceerd in Hoofdstuk 4. Stochastische simulaties laten zien dat er slechts een unipolair gepolariseerde toestand kan bestaan voor een bepaald bereik van het aantal polariteitsmoleculen. Dit wordt analytisch geverifieerd door een toy model waarin we een maximaal mogelijke bipolaire bias aanleggen. De belangrijkste conclusie is dat de invloed van geometrische signalen niet sterk genoeg zijn om de intrinsieke unipolariteit van het ontwikkelde polarisatiemechanisme te overwinnen.

De modellen die we in dit proefschrift ontwikkelen leggen een basis voor begrijpen hoe de ruimtelijke organisatie van microtubuli benvloed wordt door celvorm of polarisatiemechanismen. Bovendien kunnen ze de basis vormen van een rationele ontwerpstrategie voor het creëren van polariteit in een minimale synthetische celachtige omgeving door middel van biochemische reconstitutie.

Ter afsluiting bespreken we in Hoofdstuk 8 enkele mogelijke richtingen van toekomstig werk geïnspireerd door de huidige studie. 


\section{Acknowledgments}

First of all I want to thank my supervisor. Bela I am grateful to you for giving me the chance to work with you. You introduced me to the world of modelling cellular components and structures either by formulating dynamic equations or by applying stochastic simulations. Your great experience in Theoretical Physics made me always certain that every problem you were setting, would be solvable by using the known tools of mathematics and computer science.

Next, I thank the supervisor of my Master thesis Costas Papatriantafilloy and the Emeritus Professor Costas Paraskevaidis who both introduced me to Bela with whom they had a broad collaboration in the past. Without them this $\mathrm{PhD}$ would never have started.

I also thank Pieter-Rein for reviewing the manuscript on spherical cell polarity that later was sent to PlosOne and published.

Eva thank you for introducing me to the code that Simon wrote for the plant cortical array.

Ioanna it was fun working in the same office. Your presence was encouraging at a time when the group was counting only two members.

Bandan, thank you for the interest that you showed for my work every time that we were met at AMOLF or at the university.

Melle, Marco and Ramon I enjoyed being with you in the same group.

Katerina, thank you for the hospitality and also your encouragement at the final stages of my $\mathrm{PhD}$.

Charilaos and Giorgos thank you for being my paranymphs, carrying out all the necessary procedures of my defense.

I want to thank my wife, Markella, for her continuous support to our family and her encouragement to me. 
Acknowledgments 


\section{List of publications}

\section{Published}

- Panayiotis Foteinopoulos and Bela M. Mulder, The Effect of Anisotropic Microtubule-Bound Nucleations on Ordering in the Plant Cortical Array, Bulletin of Mathematical Biology 76(11): 29072922 (2014)

- Panayiotis Foteinopoulos and Bela M. Mulder, A microtubule-based minimal model for spontaneous and persistent spherical cell polarity, PLOS ONE 2(9): e0184706 (2017)

\section{In preparation}

- Panayiotis Foteinopoulos and Bela M. Mulder, Microtubule Organization and Cell Shape, Comprises material from Chapters 3, 4 and 7 of the present thesis. 


\section{About the author}

Panagiotis Foteinopoulos was born on May 10, 1971 in Athens, Greece. In 1989 he graduated from the 15th High school of Athens. In 1993 he obtained a diploma in Civil Engineering from the National Technical University of Athens and in 2007 a BSc in Biology from the National and Kapodistrian University of Athens. From 1994 to 2008 he made the structural design and supervision of more than 50 buildings and residences in Athens. In 2010 he obtained a MSc in Theoretical Physics and a PhD in Civil Engineering, both from the National Technical University of Athens. From January 2011 on he has been working in the AMOLF Institute, Amsterdam in the group of Prof. Dr. Bela M. Mulder. In his research he developed microtubulebased models for the establishment of polarity in cells and investigated the branched microtubule nucleation in plant cells as well as the cell shape influence on the spatial organization of microtubules. In 2014 he founded with other Greek scientists and professionals the non-profit organization 'RCR-Greece', with aim to foster research integrity in Greece. In 2015 as a president of 'RCR-Greece' he organized and hosted in Athens the Meeting of 'European Network of Research Integrity Offices' (ENRIO). For the academic year 2016-17 he had the position of Advisor Professor at the Hellenic Open University. He tought at the MSc program 'Engineering Project Management' and supervised a Master thesis. 
The work described in this thesis was performed at the Institute AMOLF, Science Park 104, 1098XG Amsterdam, The Netherlands.

Financial support from Wageningen University for printing this thesis is gratefully acknowledged.

Cover design by Markella Sifakaki. 